See discussions, stats, and author profiles for this publication at: https://www.researchgate.net/publication/324256468

\title{
Exploring cosmic origins with CORE: Inflation
}

Article in Journal of Cosmology and Astroparticle Physics · April 2018

DOI: 10.1088/1475-7516/2018/04/016

CITATIONS

80

133 authors, including:

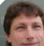

Martin Bucher

French National Centre for Scientific Research

262 PUBLICATIONS 16,601 CITATIONS

SEE PROFILE

(2.) Sebastien Clesse

(yWTH Aachen University

62 PUBLICATIONS 1,669 CITATIONS

SEE PROFILE

Some of the authors of this publication are also working on these related projects:

Project Microwave Kinetic Inductance Detecetors View project

Project VIPERS View project
READS

134

Mario Ballardini

University of the Western Cape

108 PUBLICATIONS 1,951 CITATIONS

SEE PROFILE 


\section{Exploring Cosmic Origins with CORE: Inflation}

Fabio Finelli, ${ }^{1,2}$ Martin Bucher, ${ }^{3,4}$ Ana Achúcarro, ${ }^{5,6}$ Mario Ballardini, ${ }^{7,1,2}$ Nicola Bartolo, ${ }^{8,9,10}$ Daniel Baumann, ${ }^{11,12}$ Sébastien Clesse, ${ }^{13}$ Josquin Errard, ${ }^{14}$ Will Handley, ${ }^{15,16}$ Mark Hindmarsh, ${ }^{17,18,19}$ Kimmo Kiiveri, ${ }^{18,19}$ Martin Kunz, ${ }^{20}$ Anthony Lasenby, ${ }^{15,16}$ Michele Liguori, ${ }^{8,9,10}$ Daniela Paoletti, ${ }^{1,2}$ Christophe Ringeval, ${ }^{21}$ Jussi Väliviita, ${ }^{18,19}$ Bartjan van Tent, ${ }^{22}$ Vincent Vennin, ${ }^{23}$ Peter Ade, ${ }^{24}$ Rupert Allison, ${ }^{11}$ Frederico Arroja, ${ }^{25}$ Marc Ashdown, ${ }^{16}$ A. J. Banday, ${ }^{26,27}$ Ranajoy Banerji, ${ }^{3}$ James G. Bartlett, ${ }^{3}$ Soumen Basak, ${ }^{28,29}$ Jochem Baselmans, ${ }^{30,31}$ Paolo de Bernardis, ${ }^{32}$ Marco Bersanelli, ${ }^{33}$ Anna Bonaldi, ${ }^{34}$ Julian Borril, ${ }^{35}$ François R. Bouchet, ${ }^{36}$ François Boulanger, ${ }^{37}$ Thejs Brinckmann, ${ }^{13}$ Carlo Burigana, ${ }^{1,2,38}$ Alessandro Buzzelli, ${ }^{32,39}$ Zhen-Yi Cai, ${ }^{40}$ Martino Calvo, ${ }^{41}$ Carla Sofia Carvalho, ${ }^{42}$ Gabriella Castellano, ${ }^{43}$ Anthony Challinor, ${ }^{11,16,44}$ Jens Chluba, ${ }^{34}$ Ivan Colantoni, ${ }^{43}$ Martin Crook, ${ }^{45}$ Giuseppe D'Alessandro, ${ }^{32}$ Guido D'Amico, ${ }^{46}$ Jacques Delabrouille, ${ }^{3}$ Vincent Desjacques, ${ }^{47,20}$ Gianfranco De Zotti, ${ }^{10}$ Jose Maria Diego, ${ }^{48}$ Eleonora Di Valentino, ${ }^{49,36}$ Stephen Feeney, ${ }^{50}$ James R. Fergusson, ${ }^{11}$ Raul Fernandez-Cobos, ${ }^{48}$ Simone Ferraro, ${ }^{51}$ Francesco Forastieri, ${ }^{38,52}$ Silvia Galli, ${ }^{36}$ Juan García-Bellido, ${ }^{53}$ Giancarlo de Gasperis, ${ }^{54,39}$ Ricardo T. Génova-Santos, ${ }^{55,56}$ Martina Gerbino, ${ }^{57}$ Joaquin González-Nuevo, ${ }^{58}$ Sebastian Grandis, ${ }^{59,60}$ Josh Greenslade, ${ }^{49}$ Steffen Hagstotz, ${ }^{59,60}$ Shaul Hanany, ${ }^{61}$ Dhiraj K. Hazra, ${ }^{3}$ Carlos Hernández-Monteagudo, ${ }^{62}$ Carlos Hervias-Caimapo, ${ }^{34}$ Matthew Hills, ${ }^{45}$ Eric Hivon, ${ }^{36}$ Bin Hu, ${ }^{63,64}$ Ted Kisner, ${ }^{35}$ Thomas Kitching, ${ }^{65}$ Ely D. Kovetz, ${ }^{66}$ Hannu Kurki-Suonio, ${ }^{18,19}$ Luca Lamagna, ${ }^{32}$ Massimiliano Lattanzi, ${ }^{38,52}$ Julien Lesgourgues, ${ }^{13}$ Antony Lewis, ${ }^{67}$ Valtteri Lindholm, ${ }^{18,19}$ Joanes Lizarraga, ${ }^{6}$ Marcos López-Caniego, ${ }^{68}$ Gemma Luzzi, ${ }^{32}$ Bruno Maffei, ${ }^{37}$ Nazzareno Mandolesi, ${ }^{38,1}$ Enrique Martínez-González, ${ }^{48}$ Carlos J.A.P. Martins, ${ }^{69}$ Silvia Masi, ${ }^{32}$ Darragh McCarthy, ${ }^{70}$ Sabino Matarrese, ${ }^{8,9,10,71}$ Alessandro 
Melchiorri, ${ }^{32}$ Jean-Baptiste Melin, ${ }^{72}$ Diego Molinari ${ }^{38,52}$ Alessandro Monfardini, ${ }^{73}$ Paolo Natoli, ${ }^{38,52}$ Mattia Negrello, ${ }^{74}$ Alessio Notari, ${ }^{64}$ Filippo Oppizzi, ${ }^{8}$ Alessandro Paiella, ${ }^{32}$ Enrico Pajer, ${ }^{75}$ Guillaume Patanchon, ${ }^{3}$ Subodh P. Patil, ${ }^{76}$ Michael Piat, ${ }^{3}$ Giampaolo Pisano, ${ }^{74}$ Linda Polastri, ${ }^{38,52}$ Gianluca Polenta, 77,78 Agnieszka Pollo, ${ }^{79,80}$ Vivian Poulin, ${ }^{13,81}$ Miguel Quartin, ${ }^{82}$ Andrea Ravenni, ${ }^{8}$ Mathieu Remazeilles, ${ }^{34}$ Alessandro Renzi, ${ }^{29,83}$ Diederik Roest, ${ }^{84}$ Matthieu Roman, ${ }^{14}$ Jose Alberto Rubiño-Martin, ${ }^{55,56}$ Laura Salvati, ${ }^{31}$ Alexei A. Starobinsky, ${ }^{85}$ Andrea Tartari, ${ }^{3}$ Gianmassimo Tasinato, ${ }^{86}$ Maurizio Tomasi ${ }^{33,87}$ Jesús Torrado, ${ }^{17}$ Neil Trappe, ${ }^{70}$ Tiziana Trombetti, ${ }^{1,2,38}$ Carole Tucker, ${ }^{75}$ Marco Tucci, ${ }^{20}$ Jon Urrestilla, ${ }^{6}$ Rien van de Weygaert, ${ }^{88}$ Patricio Vielva, ${ }^{48}$ Nicola Vittorio, ${ }^{54,39}$ Karl Young, ${ }^{61}$ for the CORE collaboration

${ }^{1}$ INAF/IASF Bologna, via Gobetti 101, I-40129 Bologna, Italy

${ }^{2}$ INFN, Sezione di Bologna, via Irnerio 46, I-40127 Bologna, Italy

${ }^{3}$ APC, Astroparticule et Cosmologie, Université Paris Diderot, CNRS/IN2P3, CEA/lrfu, Observatoire de Paris Sorbonne Paris Cité, 10, rue Alice Domon et Léonie Duquet, 75205 Paris Cedex 13, France

${ }^{4}$ Astrophysics and Cosmology Research Unit, School of Mathematics, Statistics and Computer Science, University of KwaZulu-Natal, Durban 4041, South Africa

${ }^{5}$ Instituut-Lorentz for Theoretical Physics, Universiteit Leiden, 2333 CA, Leiden, The Netherlands

${ }^{6}$ Department of Theoretical Physics, University of the Basque Country UPV/EHU, 48040 Bilbao, Spain

${ }^{7}$ Dipartimento di Fisica e Astronomia, Università di Bologna, Viale Berti Pichat, 6/2, I-40127 Bologna, Italy

${ }^{8}$ Dipartimento di Fisica e Astronomia "Galileo Galilei", Università degli Studi di Padova, Via Marzolo 8, I-35131, Padova, Italy

${ }^{9}$ INFN, Sezione di Padova, Via Marzolo 8, I-35131 Padova, Italy

${ }^{10}$ INAF, Osservatorio Astronomico di Padova, Vicolo dell'Osservatorio 5, I-35122 Padova, Italy

${ }^{11}$ DAMTP, Centre for Mathematical Sciences, University of Cambrige, Wilberforce Road, Cambridge, CB3 0WA, UK

${ }^{12}$ Institute of Physics, University of Amsterdam, Science Park, Amsterdam, 1090 GL, The Netherlands

${ }^{13}$ Institute for Theoretical Particle Physics and Cosmology (TTK), RWTH Aachen University, D-52056 Aachen, Germany

${ }^{14}$ Institut Lagrange, LPNHE, place Jussieu 4, 75005 Paris, France.

${ }^{15}$ Astrophysics Group, Cavendish Laboratory, Cambridge, CB3 0HE, UK

${ }^{16}$ Kavli Institute for Cosmology, Cambridge, CB3 0HA, UK

${ }^{17}$ Department of Physics and Astronomy, University of Sussex, Falmer, Brighton, BN1 9QH, UK 
${ }^{18}$ Department of Physics, Gustaf Hallstromin katu 2a, University of Helsinki, Helsinki, Finland

${ }^{19}$ Helsinki Institute of Physics, Gustaf Hallstromin katu 2, University of Helsinki, Helsinki, Finland

${ }^{20}$ Département de Physique Théorique and Center for Astroparticle Physics, Université de Genève, 24 quai Ansermet, CH-1211 Genève 4, Switzerland

${ }^{21}$ Centre for Cosmology, Particle Physics and Phenomenology, Institute of Mathematics and Physics, Louvain University, 2 chemin du Cyclotron, 1348 Louvain-la-Neuve, Belgium

${ }^{22}$ Laboratoire de Physique Théorique (UMR 8627), CNRS, Université Paris-Sud, Université Paris Saclay, Bâtiment 210, 91405 Orsay Cedex, France

${ }^{23}$ Institute of Cosmology and Gravitation, University of Portsmouth, Dennis Sciama Building, Burnaby Road, Portsmouth PO1 3FX, United Kingdom

${ }^{24}$ School of Physics and Astronomy, Cardiff University, The Parade, Cardiff CF24 3AA, UK

${ }^{25}$ Leung Center for Cosmology and Particle Astrophysics, National Taiwan University, No. 1, Sec. 4, Roosevelt Road, Taipei, 10617 Taipei, Taiwan (R.O.C.)

${ }^{26}$ Université de Toulouse, UPS-OMP, IRAP, F-31028 Toulouse Cedex 4, France

${ }^{27}$ CNRS, IRAP, 9 Av. colonel Roche, BP 44346, F-31028 Toulouse Cedex 4, France

${ }^{28}$ Department of Physics, Amrita School of Arts and Sciences, Amritapuri, Amrita Vishwa Vidyapeetham, Amrita University, Kerala 690525, India

${ }^{29}$ SISSA, Astrophysics Sector, via Bonomea 265, 34136, Trieste, Italy

${ }^{30}$ SRON (Netherlands Institute for Space Research), Sorbonnelaan 2, 3584 CA Utrecht, The Netherlands

${ }^{31}$ Terahertz Sensing Group, Delft University of Technology, Mekelweg 1, 2628 CD Delft, The Netherlands

${ }^{32}$ Physics Department "G. Marconi", University of Rome Sapienza and INFN, piazzale Aldo Moro 2, 00185, Rome, Italy

${ }^{33}$ Dipartimento di Fisica, Università degli Studi di Milano, Via Celoria 16, 20133 Milano, Italy

${ }^{34}$ Jodrell Bank Centre for Astrophysics, Alan Turing Building, School of Physics and Astronomy, The University of Manchester, Oxford Road, Manchester, M13 9PL, U.K.

${ }^{35}$ Computational Cosmology Center, Lawrence Berkeley National Laboratory, Berkeley, CA 94720, USA

${ }^{36}$ Institut d'Astrophysique de Paris, UMR7095, CNRS \& UPMC Sorbonne Universités, F-75014, Paris, France

${ }^{37}$ IAS (Institut d'Astrophysique Spatiale), Université Paris Sud, Bâtiment 12191405 Orsay, France

${ }^{38}$ Dipartimento di Fisica e Scienze della Terra, Università di Ferrara, via Saragat 1, 44122 Ferrara, Italy

${ }^{39}$ INFN Roma 2, via della Ricerca Scientifica 1, I-00133, Roma, Italy

${ }^{40}$ CAS Key Laboratory for Research in Galaxies and Cosmology, Department of Astronomy, University of Science and Technology of China, Hefei, Anhui 230026, China

${ }^{41}$ Univ. Grenoble Alpes, CEA INAC-SBT, 38000 Grenoble, France

${ }^{42}$ Institute of Astrophysics and Space Sciences, University of Lisbon, Tapada da Ajuda, 1349-018 Lisbon, Portugal 
${ }^{43}$ Istituto di Fotonica e Nanotecnologie, CNR, Via Cineto Romano 42, 00156, Roma, Italy

${ }^{44}$ Institute of Astronomy, Madingley Road, Cambridge CB3 0HA, UK

${ }^{45}$ STFC Rutherford Appleton Laboratory, Harwell Campus, Didcot OX11 0QX, UK

${ }^{46}$ Theoretical Physics Department, CERN, Geneva, Switzerland

${ }^{47}$ Physics Department, Technion, Haifa 3200003, Israel

${ }^{48}$ IFCA, Instituto de Física de Cantabria (UC-CSIC), Avenida de Los Castros s/n, 39005 Santander, Spain

${ }^{49}$ Sorbonne Universités, Institut Lagrange de Paris (ILP), F-75014, Paris, France

${ }^{50}$ Astrophysics Group, Imperial College London, Blackett Laboratory, Prince Consort Road, London, SW7 2AZ, UK

${ }^{51}$ Miller Institute for Basic Research in Science, University of California, Berkeley, CA 94720, USA

${ }^{52}$ INFN, Sezione di Ferrara, Via Saragat 1, 44122 Ferrara, Italy

${ }^{53}$ Instituto de Física Teórica UAM/CSIC, Universidad Autonoma de Madrid, 28049 Madrid, Spain

${ }^{54}$ Dipartimento di Fisica, Università di Roma "Tor Vergata", Via della Ricerca Scientifica 1, I00133, Roma, Italy

${ }^{55}$ Instituto de Astrofísica de Canarias, Calle Vía Láctea s/n, La Laguna, Tenerife, Spain

${ }^{56}$ Departamento de Astrofísica, Universidad de La Laguna (ULL), La Laguna, Tenerife, 38206 Spain

${ }^{57}$ The Oskar Klein Centre for Cosmoparticle Physics, Department of Physics, Stockholm University, AlbaNova, SE-106 91 Stockholm, Sweden

${ }^{58}$ Departamento de Física, Universidad de Oviedo, Calle Calvo Sotelo s/n, 33007 Oviedo, Spain

${ }^{59}$ Faculty of Physics, Ludwig-Maximilians Universität, 81679 Munich, Germany

${ }^{60}$ Excellence Cluster Universe, Boltzmannstrasse 2, D-85748 Garching, Germany

${ }^{61}$ School of Physics and Astronomy, University of Minnesota, 116 Church Street SE, Minneapolis, Minnesota 55455, United States

${ }^{62}$ Centro de Estudios de Física del Cosmos de Aragón (CEFCA), Plaza San Juan, 1, planta 2, E-44001, Teruel, Spain

${ }^{63}$ Department of Astronomy, Beijing Normal University, Beijing 100875, China

${ }^{64}$ Departament de Física Quàntica i Astrofísica i Institut de Ciències del Cosmos (ICCUB), Universitat de Barcelona, Martí i Franquès 1, E-08028 Barcelona, Spain

${ }^{65}$ Mullard Space Science Laboratory, University College London, Holmbury St. Mary, Darking, Surrey, RH5 6NT, UK

${ }^{66}$ Department of Physics and Astronomy, Johns Hopkins University, 3400 N. Charles St., Baltimore, MD 21218, USA

${ }^{67}$ Department of Physics and Astronomy, University of Sussex, Brighton BN1 9QH, UK

${ }^{68}$ European Space Agency, ESAC, Planck Science Office, Camino bajo del Castillo s/n, Urbanización Villafranca del Castillo, Villanueva de la Cañada, Madrid, Spain

${ }^{69}$ Centro de Astrofísica da Universidade do Porto and IA-Porto, Rua das Estrelas, 4150-762 Porto, Portugal 
${ }^{70}$ Department of Experimental Physics, Maynooth University, Maynooth, County Kildare, W23 F2H6, Ireland

${ }^{71}$ Gran Sasso Science Institute, INFN, Via F. Crispi 7, I-67100 L'Aquila, Italy

${ }^{72}$ CEA Saclay, DRF/Irfu/SPP, 91191 Gif-sur-Yvette Cedex, France

${ }^{73}$ Institut Néel CNRS/UGA UPR2940 25, rue des Martyrs BP 166, 38042 Grenoble Cedex 9, France

${ }^{74}$ School of Physics and Astronomy, Cardiff University, The Parade, Cardiff CF24 3AA, UK

${ }^{75}$ Institute for Theoretical Physics and Center for Extreme Matter and Emergent Phenomena, Utrecht University, Princetonplein 5, 3584 CC Utrecht, The Netherlands

${ }^{76}$ Niels Bohr Institute, Niels Bohr Institute, Blegdamsvej 17, Copenhagen, DK-2100, Denmark

${ }^{77}$ Agenzia Spaziale Italiana Science Data Center, via del Politecnico, 00133 Roma, Italy

${ }^{78}$ INAF, Osservatorio Astronomico di Roma, via di Frascati 33, Monte Porzio Catone, Italy

${ }^{79}$ National Centre for Nuclear Research, ul. Hoza 69, 00-681 Warszawa, Poland

${ }^{80}$ Astronomical Observatory of the Jagiellonian University, Orla 171, 30-001 Cracow, Poland

${ }^{81}$ LAPTh, Université Savoie Mont Blanc and CNRS, BP 110, F-74941 Annecy-le-Vieux Cedex, France

${ }^{82}$ Instituto de Física, Universidade Federal do Rio de Janeiro, 21941-972, Rio de Janeiro, RJ, Brazil

${ }^{83}$ INFN/National Institute for Nuclear Physics, Via Valerio 2, I-34127 Trieste, Italy

${ }^{84}$ Van Swinderen Institute for Particle Physics and Gravity, University of Groningen, Nijenborgh 4, 9747 AG Groningen, The Netherlands

${ }^{85}$ Landau Institute for Theoretical Physics RAS, Moscow 119334, Russian Federation

${ }^{86}$ Department of Physics, Swansea University, Swansea, SA2 8PP, UK

${ }^{87}$ INAF, IASF Milano, Via E. Bassini 15, Milano, Italy

${ }^{88}$ Kapteyn Astronomical Institute, University of Groningen, P.O. Box 800, 9700AV Groningen, The Netherlands

E-mail: finelli@iasfbo.inaf.it,bucher@apc.univ-paris7.fr

Abstract. We forecast the scientific capabilities to improve our understanding of cosmic inflation of CORE, a proposed CMB space satellite submitted in response to the ESA fifth call for a medium-size mission opportunity. The CORE satellite will map the CMB anisotropies in temperature and polarization in 19 frequency channels spanning the range 60-600 GHz. CORE will have an aggregate noise sensitivity of $1.7 \mu \mathrm{K}$ - arcmin and an angular resolution of 5' at $200 \mathrm{GHz}$. We explore the impact of telescope size and noise sensitivity on the inflation science return by making forecasts for several instrumental configurations. This study assumes that the lower and higher frequency channels suffice to remove foreground contaminations and complements other related studies of component separation and systematic effects, which will be reported in other papers of the series "Exploring Cosmic Origins with CORE." We forecast the capability to determine key inflationary parameters, to lower the detection limit for the tensor-to-scalar ratio down to the $10^{-3}$ level, to chart the landscape of single field slow-roll inflationary models, to constrain the epoch of reheating, thus connecting inflation to the standard radiation-matter dominated Big Bang era, 
to reconstruct the primordial power spectrum, to constrain the contribution from isocurvature perturbations to the $10^{-3}$ level, to improve constraints on the cosmic string tension to a level below the presumptive GUT scale, and to improve the current measurements of primordial nonGaussianities down to the $f_{\mathrm{NL}}^{\text {local }}<1$ level. For all the models explored, CORE alone will improve significantly on the present constraints on the physics of inflation. Its capabilities will be further enhanced by combining with complementary future cosmological observations. 


\section{Contents}

1 Introduction $\quad 1$

2 Inflation and fundamental physics $\quad 6$

$\begin{array}{lll}2.1 & \text { Physics of inflation } & 7\end{array}$

$\begin{array}{lll}2.1 .1 & \text { Ultraviolet sensitivity } & 7\end{array}$

$\begin{array}{lll}2.1 .2 & \text { Inflation in string theory } & 7\end{array}$

2.1.3 Inflation in supergravity $\quad 8$

2.1.4 Inflation in the Standard Model $\quad 8$

2.1.5 Inflation in effective field theory 9

2.2 Tensor observables $\quad 9$

2.2.1 Tensor amplitude 9

$\begin{array}{lll}2.2 .2 & \text { Tensor tilt } & 10\end{array}$

$\begin{array}{ll}2.2 .3 \text { Graviton mass } & 10\end{array}$

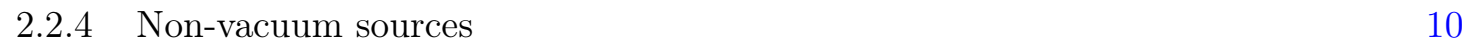

2.2.5 Non-Gaussianity 11

$\begin{array}{lll}2.3 & \text { Scalar observables } & 11\end{array}$

2.3.1 Running 11

2.3.2 Non-Gaussianity 12

$\begin{array}{lll}2.3 .3 & \text { Features } & 13\end{array}$

$\begin{array}{lll}2.3 .4 & \text { Isocurvature } & 15\end{array}$

2.4 Beginning and end of inflation $\quad 15$

2.4.1 Spatial curvature $\quad 15$

$\begin{array}{lll}2.4 .2 & \text { Topological defects } & 16\end{array}$

3 Experimental configurations and forecasting methodology 16

$\begin{array}{lll}3.1 & \text { Simplified likelihood for forecasts } & 19\end{array}$

3.2 Dealing with gravitational lensing 21

4 Probing inflationary parameters with CORE $\quad 22$

4.1 Forecasts for the spectral index and its scale dependence 22

4.2 Joint forecasts for $n_{\mathrm{s}}$ and $r \quad 26$

4.3 Beyond the consistency condition for the tensor tilt 31

4.4 Constraints on the curvature 32

$\begin{array}{lll}4.5 & \text { Summary } & 32\end{array}$

5 Constraints on slow-roll inflationary models 33

5.1 Constraints on slow-roll parameters 34

5.1.1 Impact of apparatus size and sensitivity 34

5.1.2 Removing low multipoles and delensing 36

$\begin{array}{lll}5.2 & \text { Inflationary model comparison } & 37\end{array}$ 
$\begin{array}{lll}5.3 & \text { Reheating } & 39\end{array}$

$\begin{array}{lll}5.4 & \text { Summary } & 41\end{array}$

6 Testing deviations from a power law spectrum $\quad 42$

6.1 Primordial power spectra reconstruction methodology 42

$\begin{array}{lll}6.2 & \text { Featureless scalar power spectrum } & 43\end{array}$

6.3 Scalar power spectrum with wiggles and cutoffs 44

6.4 Superimposed logarithmic oscillations 46

6.5 Reconstructing the tensor power spectrum 47

7 Testing the adiabaticity of initial conditions: constraining isocurvature $\quad 47$

$\begin{array}{lll}7.1 & \text { The model and its parameterization } & 48\end{array}$

7.2 Adiabatic fiducial data 49

7.2.1 Fitting a generally correlated mixture of adiabatic and CDI modes 49

7.2.2 Special one parameter extensions to adiabatic $\Lambda \mathrm{CDM} \quad 53$

$\begin{array}{lll}7.3 & \text { Fiducial data with a fully (anti)-correlated CDI contribution } & 54\end{array}$

$\begin{array}{ll}7.4 & \text { Adiabatic fiducial data plus a tensor contribution }\end{array}$

$\begin{array}{lll}7.5 & \text { Summary } & 59\end{array}$

8 Primordial non-Gaussianity $\quad 60$

8.1 CMB temperature and polarization bispectrum and trispectrum 60

8.1.1 Standard bispectrum shapes 60

$\begin{array}{lll}8.1 .2 & \text { Isocurvature non-Gaussianity } & 62\end{array}$

8.1.3 Spectral index of the bispectrum 64

8.1.4 Oscillatory bispectra $\quad 65$

8.1.5 Trispectrum $\quad 68$

$\begin{array}{lll}8.2 & \text { Other methods } & 68\end{array}$

$\begin{array}{lll}\text { 8.2.1 CIB power spectrum } & 70\end{array}$

$\begin{array}{lll}\text { 8.2.2 Spectral distortions } & 72\end{array}$

$\begin{array}{lll}8.3 & \text { Summary } & 74\end{array}$

9 Topological defects $\quad 75$

9.1 Calculation of CMB from defects 76

$\begin{array}{lll}9.2 & \text { MCMC Fits } & 76\end{array}$

$\begin{array}{lll}9.3 & \text { Summary } & 78\end{array}$

$\begin{array}{ll}10 \text { Conclusions } & 78\end{array}$

\section{Introduction}

Starting with the COBE detection of a cosmic microwave background (CMB) anisotropy in 1992 [1], the precision mapping of the primordial CMB anisotropies in temperature and polarization has allowed us to characterize the initial cosmological perturbations at about the percent level [2-5]. On the one hand, these observations serve as initial conditions to be used to understand 
how the highly clumpy and nonlinear universe at late times emerged. On the other hand, these observations also allow us to probe the physics of the very early universe, governed by unknown new physics at energy scales far beyond those scales that can be probed even with the most ambitious future accelerator experiments. With better observations of the CMB, we will be able to probe new physics at scales just below the Planck scale and establish meaningful constraints on theories regarding how gravity becomes unified with the other three fundamental interactions (i.e., strong, weak, and electromagnetic), presumably at an energy scale around the Planck scale.

This paper describes what we may expect to learn about cosmic inflation from future CMB experiments, in particular from the CORE mission, which is a dedicated microwave polarization satellite proposed to the European Space Agency (ESA) in October 2016 in response to a Call for proposals for a future medium-sized space mission for the "M5" launch opportunity of the ESA Cosmic Vision programme. This article, which is part of the "Exploring Cosmic Origins (ECO)" collection of articles [6-13], each describing a different aspect of the CORE mission, deals with forecasts of how the CORE data will improve our knowledge of the physics of cosmic inflation. Closely related papers include the ECO paper on cosmological parameters [9] and the ECO paper on B-mode component separation [10].

Before the recent CORE proposal, several related proposals for a post-Planck dedicated microwave polarization satellite had been submitted to ESA: B-Pol in $2007^{1}$ [14], COrE in $2011^{2}$ [15], PRISM $^{3}$ [16] in 2013 (which was a higher budget "L" (large) class mission addressing a broader, more ambitious science case), and COrE + in 2014. These proposals were highly rated but none made the final cut to selection. Similarly, in the United States, there have been several studies and proposals for similar missions. The CMB-Pol mission concept study produced a number of detailed white papers, one of which deals with inflation [17] and thus has much overlap with this work, and also specialized white papers on foregrounds [18, 19] and gravitational lensing [20], as well as a general overview paper [21]. EPIC was proposed to NASA in 2008. The EPIC study $[22,23]$ presents a detailed conceptual design in the form of three options: a low-cost option, an intermediate option, and a comprehensive science option. Another US initiative is the proposed NASA PIXIE mission [24], which would map the microwave sky using a Fourier Transform Spectrometer (FTS) much like the COBE FIRAS instrument [25] but two orders of magnitude more sensitive and with sensitivity to polarization. A modified version of PIXIE [26] was proposed for the NASA MIDEX call 2016 and could potentially be launched in 2023. In Japan a CMB polarization space mission called LiteBIRD [27, 28] is presently undergoing a Phase A study together with NASA. Compared to CORE, LiteBIRD has a smaller aperture, thus limiting its reach toward small angular scales. It is a lower cost mission with an earlier planned launch, according to the present schedule around 2025.

When inflationary cosmology was introduced in the early 1980s [29-36], it was initially greeted with great interest accompanied by a healthy dose of skepticism. In the few years following the COBE discovery, it was not at all obvious that inflation would survive a confrontation with forthcoming data. However, with the first clear observations of the first acoustic peaks [37, 38], followed by the mapping of the three acoustic peaks by WMAP [39], and subsequently by the

\footnotetext{
${ }^{1}$ See www.b-pol.org for a copy of the proposal and more details.

${ }^{2}$ www.core-mission.org

${ }^{3}$ www.prism-mission.org
} 
precision mapping of the five peaks by the ESA Planck mission [2], many of the competing models of structure formation fell by the wayside, and it turned out that rather simple models of inflation could account for the data $[3,5]$.

The plethora of disparate competing cosmological models existing at the time of the COBE discovery gradually became replaced with what has now become known as the 'concordance' model of cosmology. More precisely, this is the six-parameter $\Lambda$ CDM model described in detail in the WMAP and Planck papers dedicated to making the connection between the CMB observations and cosmological models [2, 4]. For the purposes of the present paper, it suffices to highlight the following key points:

(1) WMAP and Planck found that the data can be explained by a six-parameter $\Lambda \mathrm{CDM}$ cosmological model under which the scalar power spectrum takes the following simple power law form

$$
\mathcal{P}_{\mathcal{R}}(k)=A_{\mathrm{s}}\left(\frac{k}{k_{*}}\right)^{n_{\mathrm{s}}-1}
$$

where $k_{*}$ is a pivot scale (unless otherwise stated fixed to $0.05 \mathrm{Mpc}^{-1}$ ). This model also includes four additional non-inflationary parameters $H_{0}, \omega_{\mathrm{b}}=\Omega_{\mathrm{b}} h^{2}, \omega_{\mathrm{c}}=\Omega_{\mathrm{c}} h^{2}$, and $\tau$. The model provides a good fit to the data: there is no statistically significant evidence compelling us to extend this model despite the many extensions that have been explored [2-5]. The TT, TE, and EE CMB power spectra, at present most tightly constrained by the Planck data, and on smaller angular scales by data from ACT [40] and SPT [41], are well accounted for by this model, which moreover is broadly consistent with other probes such as Baryon Acoustic Oscillations (BAO) [42, 43] and constraints on $\omega_{\mathrm{b}}$ derived from the observed light element abundances interpreted using the theory of primordial big bang nucleosynthesis. Planck data alone have been able to set limits at the percent level on the curvature [44, 45], verifying one of the basic predictions of the simplest inflationary models. Among the caveats to an interpretation based on $\Lambda \mathrm{CDM}$ are disagreements at modest statistical significance, sometimes euphemistically dubbed 'tensions' with determinations of $H_{0}$ [46] and with cosmological parameters determined using cluster abundances [47, 48] or galaxy shear measurements $[49,50]$. Importantly, the model includes only the adiabatic growing mode for primordial fluctuations as predicted by inflation driven by a single scalar field. No statistically significant evidence was uncovered showing that isocurvature modes were excited $[3,5]$, which is possible in multi-field inflationary models.

(2) One of the most significant findings, first made by WMAP at modest statistical significance and later by Planck at much higher statistical significance, was that the primordial power spectrum is not exactly scale invariant: in other words, $n_{\mathrm{s}} \neq 1[3,5]$. This finding is consistent with those inflationary models which have a natural exit from inflation.

(3) Another far reaching result of the ESA Planck mission was the tight constraints established on primordial non-Gaussianity [51, 52]. These upper bounds rule out at high statistical significance many of the non-standard inflationary models predicting a level non-Gaussianity allowed by WMAP [53].

(4) With the presently available CMB data, the scalar power spectrum as given in Eq. (1.1) has been mapped out over approximately three decades in wavenumber. But at present, apart from upper bounds [54, 55], almost nothing is known about the tensor mode power spectrum. That tensor modes should be excited is one of the most remarkable and surprising predictions of 

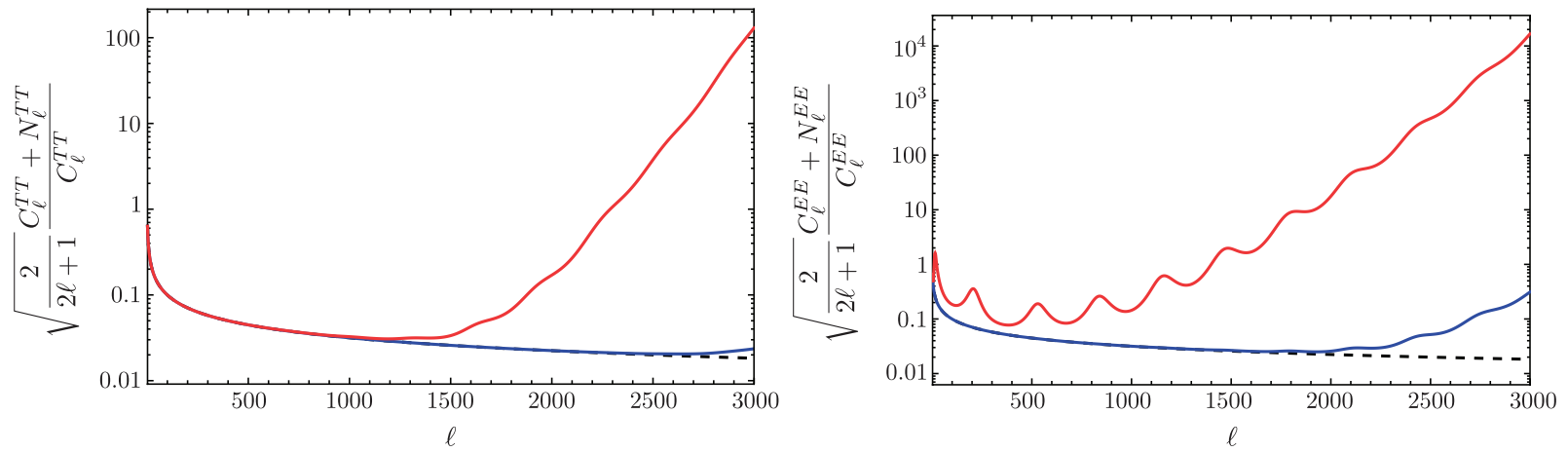

Figure 1: The improvement in $T T$ (left panel) and $E E$ (right) power spectra as a function of the multipole number $\ell$ for Planck (red line) and CORE (blue line) up to $\ell \approx 3000$ compared to the cosmic variance limit with $f_{\text {sky }}=1$ (dashed black line).

cosmic inflation. Yet this prediction has not yet been tested. Discovering primordial B modes from inflation is the primary goal of almost all future CMB experiments, as we detail below.

In order to provide a very approximate idea of how much more cosmologically relevant data the CORE satellite will collect compared to the data already available from Planck, we examine how much the error bars on the underlying theoretical power spectra will shrink as the result of the addition of future CORE data. In this analysis we suppose that the underlying stochastic process is nearly Gaussian, an assumption consistent with the failure of Planck to turn up any statistically significant evidence for primordial non-Gaussianity [51, 52]. The precise likelihood for a given theoretical power spectrum for a realistic survey is complicated, but the following analytic order of magnitude estimate suffices [56]:

$$
\left(\frac{\delta C_{\ell}}{C_{\ell}}\right)_{\mathrm{rms}} \approx \sqrt{\frac{2}{f_{\mathrm{sky}}(2 \ell+1)}} \frac{C_{\ell}+N_{\ell}}{C_{\ell}},
$$

where $f_{\text {sky }}$ is the sky fraction surveyed, $C_{\ell}$ refers to the power spectrum, and $N_{\ell}$ is the measurement noise. There are two regimes to consider. When $N_{\ell} \lesssim C_{\ell}$, which is very much the case at low $\ell$ for $C_{\ell}^{T T}$, the uncertainty is dominated by "cosmic variance," and in terms of fixing the power spectrum, reducing $N_{\ell}$ further is of marginal added value. Our inability to fix the power spectrum of the underlying stochastic process arises primarily because we can observe only one sky. In the regime $N_{\ell} \ll C_{\ell}$, the microwave sky has been mapped sufficiently well, so there is little motivation to construct a less noisy map. On the other hand, in the multipole range where $N_{\ell} \gtrsim C_{\ell}$, there remains significant new information to be gained, and the error bars on the underlying CMB power spectra can be shrunk down further by producing better maps based on new data. Fig. 1 shows the improvement that can be reached with CORE over Planck in temperature and polarization according to the analytical estimate in Eq. (1.2), showing that CORE will make cosmic variance limited measurements of the TT power spectrum for $\ell \lesssim 2500$ and for $\ell \lesssim 2000$ for $E E$.

The effective number of modes measured by a given survey is

$$
N_{\text {modes }}=\sum_{\ell, m}\left(\frac{C_{\ell}}{C_{\ell}+N_{\ell}}\right)^{2} .
$$



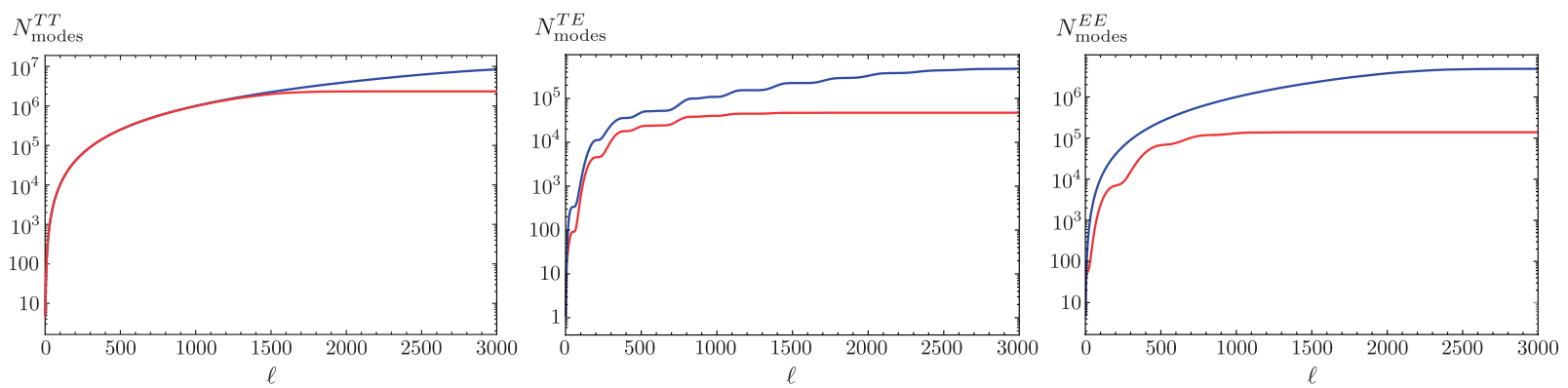

Figure 2: The effective number of modes defined in Eq. (1.3) for TT (left panel), TE (middle panel), $E E$ (right panel) as a function of the multipole number $\ell$ for Planck (red line) and CORE (blue line) up to $\ell \approx 3000$.

For each of the power spectra $C_{\ell}^{T T}, C_{\ell}^{T E}$, and $C_{\ell}^{E E}$, we determine how many new modes will be measured by the various configurations of CORE compared to the existing data from Planck. This analysis makes sense when we already have a good measurement of underlying power spectra. We therefore restrict to $C_{\ell}^{T T}, C_{\ell}^{T E}$, and $C_{\ell}^{E E}$, since the primordial $C_{\ell}^{B B}$ from inflationary gravitational waves is still unknown. Fig. 2 plots the effective number of modes for the $T T, T E$, and $E E$ spectra for Planck and CORE up to $\ell \approx 3000$. We observe that for measuring the $E E$ power spectrum, a lot remains to be gained from a more sensitive survey, especially at high $\ell$. We also note that interesting room remains for improving our knowledge of the $T T$ power spectrum on smaller scales, but our ability to remove secondary anisotropies and foreground residuals rather than the noise is the limitation to extracting new information regarding primordial anisotropies in this region. CORE has a great potential to enhance our knowledge of extragalactic sources [11] and therefore to better characterize the CMB high- $\ell$ damping tail of temperature anisotropies. See also Ref. [57] for a recent paper studying the CORE capabilities to advance our understanding of the anisotropies of the cosmic far-infrared background.

The previous discussion has emphasized the scientific objective of measuring the primordial power spectrum. But this style of analysis can also be applied to forecasting how much various non-Gaussian analyses will improve when the data from CORE is used instead of the less accurate existing Planck data. As a concrete example, consider the constraints on local bispectral nonGaussianity as predicted by many non-minimal inflationary models such as those having more than one scalar field. An approximate analytic expression for the information constraining the parameter $f_{\mathrm{NL}}^{\text {local }}$ in a sky map spanning the multipole range $\left[\ell_{\min }, \ell_{\max }\right]$ at a signal-to-noise ratio equal to or larger than one is given by [58]

$$
O(1)\left(f_{\mathrm{NL}}^{\text {local }}\right)^{2} \ell_{\max }^{2} \ln \left(\frac{\ell_{\max }}{\ell_{\min }}\right)
$$

where the presence of the logarithmic factor emphasizes the importance of full sky coverage as one typically obtains from a space-based experiment. The bottleneck for improving on Planck arises from the $\ell_{\max }{ }^{2}$ factor, which is proportional to the number of modes as defined in Eq. (1.3).

The purpose of this paper is to study the capabilities of the ESA M5 mission proposal CORE and to compare them with those obtained from other designs for CMB space missions, such as 
the JAXA LiteBIRD configuration, the LiteCore designs, and the CORE + proposal submitted for the previous ESA M4 mission call. This comparison sheds light on the role of different angular resolutions and raw detector sensitivities on the constraints on inflation to be expected from a future CMB space mission.

While space provides the most hospitable environment for searching for primordial B modes, a number of ground based and balloon experiments also seeking to detect B modes are now either underway or in the planning stage. These include Keck Array/BICEP 3/Bicep Array [59], Spider [60], POLARBEAR-2 and the Simons Array [61], Advanced ACTpol [62], SPT-3G [63], Piper [64], CLASS [65], LSPE [66], QUIJOTE [67] and the US DOE Stage 4 (S4) experiment [68]. Although capable of a much finer angular resolution, ground based experiments must overcome a number of limitations absent for experiments deployed in space, such as atmospheric emission and absorption, ground pickup through beam far sidelobes, unstable time-varying observing conditions and limitations to sky and frequency coverage. In particular, the channels most difficult to access from the ground are those at high frequencies where the polarized dust emission is most intense. The role of polarized dust emission, first measured by Planck at high frequencies from space, has been shown to be of key importance not only for the correct interpretation [54] of the B-mode detection by BICEP 2 [69], but also for a more accurate determination of the optical depth from E-mode polarization [70-73].

We stress however the complementarity of CORE and S4. One example of such complementarity is a more efficient delensing of the primordial B modes. Although CORE will be able to delens using its own data alone, combining with data from $\mathrm{S} 4$ hold promise to delens down to lower values of $r$. Likewise, the maps from CORE at frequencies inaccessible from the ground are likely to provide invaluable information for the $\mathrm{S} 4$ analysis.

The organization of the paper is as follows. Section 2 presents the connection between inflation and fundamental physics. Section 3 describes the methodology used for forecasting the performance of CORE and of other alternative configurations. In Section 4, the forecasts for key inflationary parameters such as the scalar tilt and its scale dependence, the tensor-to-scalar ratio, and the spatial curvature are presented. The expected constraints on slow-roll parameters and a Bayesian comparison among slow-roll inflationary parameters are discussed in Section 5. In Section 6, the forecasts for spectrum reconstruction with CORE are explored. Here a nonparametric analysis attempting to find statistically significant features in the primordial power spectrum is considered. Section 7 discusses tests of the adiabaticity of the primordial fluctuations based on searching for primordial isocurvature modes. The expected constraints on primordial nonGaussianities are studied in Section 8. Section 9 forecasts the expected constraints on topological defect models. Finally, Section 10 presents some conclusions.

\section{Inflation and fundamental physics}

At the current level of sensitivity, the initial conditions of the universe are described by just two numbers: the amplitude of primordial curvature perturbations $A_{\mathrm{s}}$ and its spectral index $n_{\mathrm{s}}$. Moreover, the form of the power spectrum follows from the weakly broken scaling symmetry of the inflationary spacetime and is therefore rather generic. For these reasons, it is hard to extract detailed information about the microphysical origin of inflation from current observations. With 
future observations, we hope to detect extensions of the simple two-parameter description of the initial conditions (see Table 1). As we will describe in this section, these observations have the potential to reveal much more about the physics of the inflationary era.

\subsection{Physics of inflation}

\subsubsection{Ultraviolet sensitivity}

It is rare that our understanding of Planck-scale physics matters for the description of lowenergy phenomena. This is because even large changes in the couplings to Planck-scale degrees of freedom usually have small effects on observables at much lower energies. Quantum gravity is irrelevant (in the technical sense) for experiments at the LHC. It is therefore a remarkable feature of inflation that it is sensitive to the structure of the theory at the Planck scale. Orderone changes in the interactions with Planck-scale degrees of freedom generically have significant effects on the inflationary dynamics. As we will see, this ultraviolet (UV) sensitivity is especially strong in models with observable levels of gravitational waves. Writing down a theory of inflation therefore requires either making strong assumptions about the UV embedding, or formulating the theory in a UV-complete framework. On the other hand, the UV sensitivity of inflation is also an opportunity to learn about the nature of quantum gravity from future cosmological observations.

\subsubsection{Inflation in string theory}

String theory remains the most promising framework for addressing the issues raised by the UV sensitivity of the inflationary dynamics. The question of consistency with quantum gravity is particularly pressing in models of large-field inflation with observable levels of gravitational waves (see Sec. 2.2.1). Effective field theory (EFT) models of large-field inflation have to assume protective internal symmetries for the inflaton field in order to forbid dangerous UV corrections. Such symmetries are generically broken in quantum gravity, so it is unclear whether the success of

\begin{tabular}{cllc}
\hline Parameter & Meaning & Physical Origin & Current Status \\
\hline$A_{\mathrm{s}}$ & Scalar amplitude & $H, \dot{H}, c_{\mathrm{s}}$ & $(2.13 \pm 0.05) \times 10^{-9}$ \\
$n_{\mathrm{s}}$ & Scalar tilt & $\dot{H}, \ddot{H}, \dot{c}_{\mathrm{s}}$ & $0.965 \pm 0.005$ \\
\hline $\mathrm{d} n_{\mathrm{s}} / \mathrm{d} \ln k$ & Scalar running & $\dddot{H}, \ddot{c}_{\mathrm{s}}$ & only upper limits \\
$A_{\mathrm{t}}$ & Tensor amplitude & $H$ & only upper limits \\
$n_{\mathrm{t}}$ & Tensor tilt & $\dot{H}$ & only upper limits \\
$r$ & Tensor-to-scalar ratio & $\dot{H}, c_{\mathrm{s}}$ & only upper limits \\
$\Omega_{\mathrm{k}}$ & Curvature & Initial conditions & only upper limits \\
$f_{\mathrm{NL}}$ & Non-Gaussianity & Extra fields, sound speed, $\cdots$ & only upper limits \\
$S$ & Isocurvature & Extra fields & only upper limits \\
$G \mu$ & Topological defects & End of inflation & only upper limits \\
\hline
\end{tabular}

Table 1: Summary of key parameters in inflationary cosmology, together with their likely physical origins and current observational constraints. At present, only upper limits exist for all parameters except $A_{\mathrm{s}}$ and $n_{\mathrm{s}}[5]$. 
an EFT realization of large-field inflation survives its embedding into a UV-complete framework. In string theory these abstract questions can in principle be addressed by concrete computations. One of the main advances in the field were the first semi-realistic models of large-field inflation in string theory $[74,75]$ (see also [76, 77]). Although work remains to be done to scrutinize the details of these models, they provide the first concrete attempts to study the UV sensitivity of large-field inflation directly.

\subsubsection{Inflation in supergravity}

Another interesting question concerns the realization of inflation in supergravity, which is an intermediate platform between top-down string theory and bottom-up effective field theory approaches. The possible inflationary dynamics and couplings to other fields are then restricted by local supersymmetry. At the two-derivative level, the scalar fields of $\mathcal{N}=1$ supergravity span a so-called Kähler manifold, while the potential energy is dictated by an underlying superpotential.

The literature of inflation in supergravity is vast, so we here restrict ourselves to a few comments on recent developments. In recent years, various ways have been found to realize the Starobinsky model [30] in supergravity [78-82] providing a natural target of future B-mode searches. Moreover, a large class of supergravity models was shown to draw its main properties from the Kähler geometry. For instance, the stability of inflationary models is determined by specific components of the scalars curvature, see e.g. [83, 84]. Similarly, their inflationary predictions follow from the curvature rather than by their potential. The latter type of models are referred to as $\alpha$-attractors [85], and give a spectral index in excellent agreement with the latest Planck data. Moreover, the level of tensor modes is directly related to the curvature $R_{K}$ of the hyperbolic manifold (in Planck units)

$$
r=\frac{8}{\left(-R_{K}\right) N^{2}}
$$

where $N=O(60)$ indicates the total number of $e$-folds of the observable part of the inflationary epoch. More generally, bounds on the curvature tensor of the Kähler manifold [83, 84] imply an interesting constraint on the sound speed $c_{s} \gtrsim 0.4$ for $\mathcal{N}=1$ supergravity models in which a single chiral superfield evolves during inflation, suggesting that this scenario can be constrained by a measurement of non-Gaussianity [86].

Finally, in addition to linearly realized supersymmetry, non-linear realizations have been proposed. Non-linear realizations offer a number of phenomenological simplifications, such as the absence of possibly tachyonic directions [87]. They can be regarded as effective descriptions when supersymmetry is spontaneously broken, as occurs during inflation. Examples of supersymmetric effective field theories of inflation are [88-91].

\subsubsection{Inflation in the Standard Model}

To date, only one scalar field has been observed directly: the Standard Model (SM) Higgs field. Simplicity compels us to consider this as a possible inflaton candidate. Confined to SM interactions alone, the potential of the Higgs singlet is never flat enough to inflate. However, if the Higgs couples non-minimally to gravity, then the kinetic mixing with the graviton results in an exponentially flat potential at large enough field values [92]. With enough assumptions about 
running in the intermediate field regime, one then makes predictions for CMB observables in terms of SM parameters at low energies [92, 93]. The tensor-to-scalar ratio predicted by these models is $r \sim 10^{-3}$.

\subsubsection{Inflation in effective field theory}

The most conservative way of describing inflationary observables is in terms of an effective theory of adiabatic fluctuations [94]. Given an expansion history defined by the time-dependent Hubble rate $H(t)$, fluctuations are described by the Goldstone boson $\pi$ associated with the spontaneously broken time translation symmetry. In the absence of additional light degrees of freedom, the Goldstone boson is related in a simple way to the comoving curvature perturbation $\mathcal{R}=-H \pi$. At quadratic order in fluctuations and to lowest order in derivatives, the effective action for $\pi$ contains two time-dependent parameters: $\epsilon_{1}(t) \equiv-\dot{H} / H^{2}$ and $c_{\mathrm{S}}(t)$. The latter characterizes the sound speed of $\pi$ fluctuations. The amplitude of the power spectrum of curvature perturbations then is

$$
A_{\mathrm{S}}=\frac{1}{8 \pi^{2}} \frac{1}{\epsilon_{1} c_{\mathrm{S}}} \frac{H^{2}}{M_{\mathrm{pl}}^{2}} .
$$

The near scale-invariance of the power spectrum requires the time dependence of $\epsilon_{1}(t)$ and $c_{s}(t)$ to be mild. Interestingly, the nonlinearly realized time translation symmetry relates a small value of $c_{\mathrm{S}}$ to a cubic operator in the action for $\pi$, leading to enhanced non-Gaussianity of the fluctuations with $f_{\mathrm{NL}} \sim c_{\mathrm{S}}^{-2}$ (see Sec. 2.3.2). Additional higher-order operators of the effective action for $\pi$ are associated with additional free parameters.

\subsection{Tensor observables}

The most important untested prediction of inflation concerns the existence of tensor modes, arising from quantum fluctuations of the metric. A detection would have a tremendous impact as it would be the first experimental signature of quantum gravity.

\subsubsection{Tensor amplitude}

The amplitude of inflationary tensor modes is typically expressed in terms of the tensor-to-scalar ratio $r \equiv A_{\mathrm{t}} / A_{\mathrm{s}}$. The parameter $r$ provides a measure of the expansion rate during inflation

$$
H=7.2 \times 10^{12} \mathrm{GeV}\left(\frac{r}{0.001}\right)^{1 / 2}
$$

which can be related to the energy scale of inflation, $\rho^{1 / 4}=6.1 \times 10^{15} \mathrm{GeV}(r / 0.001)^{1 / 4}$. The observation of primordial tensor modes at the level $r>0.001$ would therefore associate inflation with physics at the GUT scale.

Although there is no definitive prediction for the magnitude of $r$, there exist simple arguments why $r>0.001$ is a theoretically interesting observational target:

- Famously, for inflationary models driven by a fundamental scalar field, the value of $r$ is related to the total field excursion [95, 96]

$$
\frac{\Delta \phi}{M_{\mathrm{Pl}}} \approx \frac{N}{90}\left(\frac{r}{0.001}\right)^{1 / 2}
$$


An observation of tensor modes above the per thousand level would therefore imply largefield inflation with super-Planckian field excursions.

- A well-motivated ansatz for the inflationary observables, satisfied by a large class of inflationary models, corresponds to an expansion in $1 / N$ with leading terms [97-101]

$$
n_{\mathrm{s}}=1-\frac{p}{N}, \quad r=\frac{r_{0}}{N^{q}} .
$$

Interestingly, this simple scaling leads to two universality classes. The first has $q=1$ and $r_{0}=8(p-1)$, comparable to quadratic inflation which is already under serious tension and will be probed further with ground-based experiments. The second has $p=q$, comparable to Starobinsky inflation and $\alpha$-attractors $[30,85]$ and leads to a per thousand level of $r$.

The above two arguments make the range between $10^{-3}$ and $10^{-2}$, which includes a variety of specific models [102], a theoretically interesting regime for the tensor-to-scalar ratio.

\subsubsection{Tensor tilt}

In the event of a detection of primordial tensor modes, it will be interesting to probe the scale dependence of the spectrum. In standard single-field slow-roll inflation, the tensor tilt satisfies a consistency relation, $n_{\mathrm{t}}=-r / 8$. Unfortunately, this makes the expected tensor tilt too small to be detectable with future CMB experiments. Nevertheless, it remains interesting to look for larger deviations from the consistency conditions. It would be striking to find a blue tensor tilt $n_{\mathrm{t}}>0$, which in the context of inflation would require a violation of the null energy condition. ${ }^{4}$

\subsubsection{Graviton mass}

If the graviton has a mass $m_{g}$, the dispersion relation of gravitational waves is modified: $\omega^{2}=$ $k^{2}+m_{g}^{2}$. For masses comparable to the Hubble rate at recombination this has a significant effect on the B-mode spectrum. In that case, the tensor mode oscillates on superhorizon scales which adds power to the B-mode spectrum on large angular scales $(\ell<100)$. Observing primordial B-modes but not finding this excess in large-scale power would put a strong constraint on the graviton mass, $m_{g}<10^{-30} \mathrm{eV}$ [111] (compared to $m_{g}<1.2 \times 10^{-22} \mathrm{eV}$ from LIGO [112]).

\subsubsection{Non-vacuum sources}

So far we have assumed that the primordial tensor modes are generated by vacuum fluctuations. In principle, there could also be tensor modes produced by non-vacuum fluctuations, such as the

\footnotetext{
${ }^{4} \mathrm{~A}$ blue tensor tilt can arise if large curvature corrections during inflation lead to a tensor sound speed with non-trivial evolution [103]. In Einstein frame, this effect would correspond to a stable violation of the null energy condition as discussed in [104, 105] (see also [106, 107]). However, it is hard to make this effect large without awakening ghosts in the effective theory. Alternatively, a blue tensor tilt can also arise in models with a nonstandard spacetime symmetry breaking pattern, such as solid [108] or supersolid [109] inflation. In these scenarios, spatial reparameterization invariance is spontaneously broken during inflation, by means of background fields with space-dependent vacuum expectation values. Since spatial diffeomorphism invariance is broken, there is no symmetry preventing the tensor modes from acquiring an effective mass during inflation [110]. The graviton mass, if sufficiently large, can lead to a blue spectrum for primordial tensor modes, as explicitly shown in concrete realizations in $[108,109]$. After inflation ends, the field configuration can rearrange itself so as to recover space reparameterization symmetry and set the effective graviton mass equal to zero.
} 
fluctuations that could arise from particle production during inflation [113-116]. One may be concerned that this could destroy the relationship between the size of $r$ and the energy scale of inflation. However, it has been shown that non-vacuum fluctuations cannot be parametrically larger than vacuum fluctuations without violating bounds on primordial non-Gaussianity [114]. The B-mode amplitude therefore remains a good measure of the energy scale of inflation.

\subsubsection{Non-Gaussianity}

If the amplitude of primordial tensors is large, it may become feasible to study higher-order correlators involving tensor modes. Of particular interest is the tensor-scalar-scalar bispectrum $\langle h \mathcal{R} \mathcal{R}\rangle$. In single-field inflation, the form of the squeezed limit of this bispectrum is fixed by the fact that a long wavelength tensor fluctuation is locally equivalent to a spatially anisotropic coordinate transformation [117]. This consistency condition is more robust that the corresponding consistency condition for the scalar correlator $\langle\mathcal{R} \mathcal{R} \mathcal{R}\rangle$ in the sense that it cannot be violated by the presence of additional scalar fields. Observing non-analytic corrections to the consistency condition for $\langle h \mathcal{R} \mathcal{R}\rangle$ (e.g., through a measurement of $\langle B T T\rangle$ [118]) would be a signature of extra higher-spin particles during inflation $[119,120]$ or of a different symmetry breaking pattern in the EFT of inflation [108, 109, 121-124]. ${ }^{5}$

\subsection{Scalar observables}

Future CMB observations will improve constraints on primordial scalar fluctuations through precision measurements of the damping tail of the temperature anisotropies and the polarization of the anisotropies. In this Section, we will describe what can be learned from these measurements.

\subsubsection{Running}

Assuming that deviations of the primordial power spectrum from a perfect power law are small, they can be parameterized with the running of the spectral tilt $\mathrm{d} n_{\mathrm{s}} / \mathrm{d} \ln k$. Current constraints from Planck give $\mathrm{d} n_{\mathrm{s}} / \mathrm{d} \ln k=-0.0057_{-0.0070}^{+0.0071}(68 \% \mathrm{CL})$ [5]. The standard prediction of single-field inflation is conveniently written in terms of observables as [126]

$$
\frac{\mathrm{d} n_{\mathrm{s}}}{\mathrm{d} \ln k}=\left(1-n_{\mathrm{s}}\right)^{2}-6 \epsilon_{1}\left(1-n_{\mathrm{s}}\right)+8 \epsilon_{1}^{2}-\left(\frac{r s}{8 c_{\mathrm{s}}}+\frac{r_{, N N}}{r}\right)
$$

where $r=16 \epsilon_{1} c_{\mathrm{s}}, s \equiv c_{\mathrm{s}, N} / c_{\mathrm{S}}$ and $*, N$ refers to a derivative with respect to the number of $e$-foldings from the end of inflation, namely $H \mathrm{~d} t=-\mathrm{d} N$. Barring cancellations, one expects $\mathrm{d} n_{\mathrm{s}} / \mathrm{d} \ln k \sim\left(1-n_{\mathrm{s}}\right)^{2}$. While $\epsilon_{1}$ is related to $r$ once $c_{s}$ is known or constrained and $n_{\mathrm{s}}$ is

\footnotetext{
${ }^{5}$ Models with space-dependent background fields have a symmetry breaking pattern different from standard single-field inflation and can lead to scenarios in which inflation is not a strong isotropic attractor. In this case, anisotropies are not diluted exponentially fast by inflation. This implies that tensor fluctuations are not adiabatic during inflation and that violations of consistency relations in the tensor-scalar-scalar correlation functions are generally expected [121]. In these models, the squeezed limit for the $\langle h \mathcal{R} \mathcal{R}\rangle$ three-point function can have an amplitude much larger than in standard inflationary scenarios [109, 125]. In addition, such non-standard behavior for the tensor modes leads to indirect, distinctive consequences for correlation functions of the scalar perturbation, namely a quadrupolar contribution to the scalar two-point function $\langle\mathcal{R} \mathcal{R}\rangle$ and a direction-dependent contribution to the counter-collinear limit of the four-point function $\langle\mathcal{R} \mathcal{R} \mathcal{R} \mathcal{R}\rangle[121]$.
} 


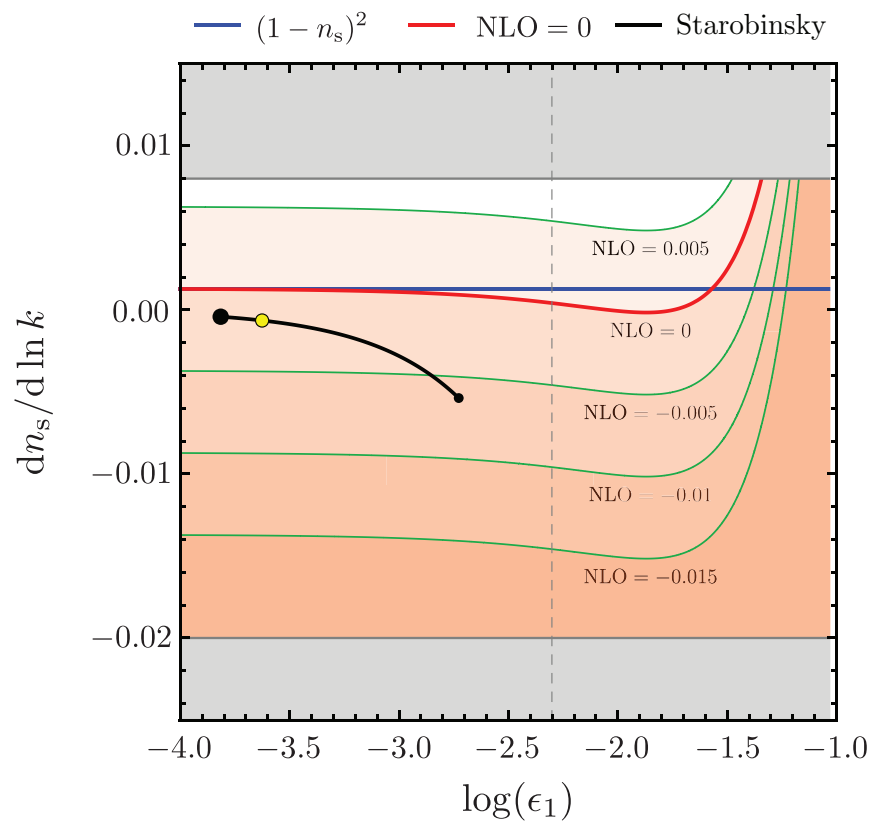

Figure 3: The plot [126] shows the running $\mathrm{d} n_{\mathrm{s}} / \mathrm{d} \ln k$ as function of $\epsilon_{1}$ for different values of the NLO slow-roll parameters. Notice that the uncertainty in $n_{\mathrm{s}}$ is smaller than the thickness of the lines in the plot. In red we show $\mathrm{d} n_{\mathrm{s}} / \mathrm{d} \ln k$ for $\mathrm{NLO}=0$, while the blue line is its asymptotic value $\left(1-n_{\mathrm{s}}\right)^{2} \approx 0.0013$. The black line shows the predictions of the Starobinsky model [30] (with $N$ going from 20 to 70 ), with the yellow dot being its prediction for $N=56$ (chosen to reproduce the observed value of $n_{\mathrm{s}}$ ). The gray bands show the values of $\mathrm{d} n_{\mathrm{s}} / \mathrm{d} \ln k$ excluded (at $95 \% \mathrm{CL}$ ) by Planck TT, TE, EE + lowP data, while the gray dashed vertical line shows the current bound on $\epsilon_{1}=r /\left(16 c_{\mathrm{s}}\right)$ assuming $c_{\mathrm{s}}=1$.

measured, the last two terms in (2.6) make their first appearance in $\mathrm{d} n_{\mathrm{s}} / \mathrm{d} \ln k$. In this sense they are next-to-leading order (NLO) parameters

$$
\mathrm{NLO} \equiv \frac{r s}{8 c_{\mathrm{s}}}+\frac{r_{, N N}}{r} \stackrel{c_{\mathrm{s}}=1}{\longrightarrow} \frac{\epsilon_{1, N N}}{\epsilon_{1}} .
$$

Since $n_{\mathrm{s}}$ is relatively well constrained, it is convenient to summarize current and future constraints in terms of $\epsilon_{1}$ and $\mathrm{d} n_{\mathrm{s}} / \mathrm{d} \ln k$ as in Fig. 3 (from [126]).

Deviations from a power-law behavior can be expanded one order further to include the running of the running $\mathrm{d}^{2} n_{\mathrm{s}} / \mathrm{d} \ln k^{2}$. Current constraints from Planck give $\mathrm{d}^{2} n_{\mathrm{s}} / \mathrm{d} \ln k^{2}=0.025 \pm 0.013$ $(68 \%$ CL) [5]. A potential detection of the running of running with CORE would be in conflict with the single-field, slow-roll paradigm, which generally predicts a much smaller value of order $\left(1-n_{\mathrm{s}}\right)^{3}$.

\subsubsection{Non-Gaussianity}

In standard single-field slow-roll inflation, the flatness of the inflaton potential constrains the size of interactions in the inflaton fluctuations. These inflaton fluctuations are therefore expected to be highly Gaussian. Significant non-Gaussianity in the initial conditions can nevertheless arise in simple extensions of the standard single-field slow-roll paradigm.

Sound speed Higher-derivative inflaton interactions have been proposed as a mechanism to slow down the inflaton evolution even in the presence of a steep potential [127]. A consequence of these interactions are a reduced sound speed $^{6}$ for the inflaton interactions which leads to a significant level of non-Gaussianity peaked in the equilateral configuration [128]. In the framework of the EFT of inflation [94], the relation between small $c_{\mathrm{s}}$ and large $f_{\mathrm{NL}} \sim c_{\mathrm{S}}^{-2}$ is a consequence

\footnotetext{
${ }^{6}$ In Dirac-Born-Infeld (DBI) inflation [127] significant reductions in the speed of sound are possible and radiatively stable due to a nonlinearly realized Lorentz symmetry protecting the structure of the DBI action.
} 
of the nonlinearly realized time translation symmetry. Current constraints on equilateral nonGaussianity imply $c_{\mathrm{s}}>0.024$ [52]. This is still an order of magnitude away from the unitarity bound derived in [129].

CORE will also constrain the closely related class of single-field slow-roll models in which the speed of sound of the inflaton fluctuations is not much less than unity and slowly varying. ${ }^{7}$ In general, these models have lower values of $r=16 \epsilon_{1} c_{\mathrm{s}}$ than their $c_{\mathrm{s}}=1$ counterparts, but the reductions are moderate. If the speed of sound is approximately constant, unitarity implies a lower bound $c_{s} \gtrsim 0.3$ in the absence of protective symmetries [129, 130]. Probing $r$ down to $10^{-3}$ will constrain, and in some cases rule out any such deformations of the 'vanilla' slow-roll models [131].

Extra fields Non-Gaussianity is a powerful way to detect the presence of extra fields during inflation. The self-interactions of these fields are not as strongly constrained as those of the inflaton. Non-Gaussianity in these hidden sectors can then be converted into observable non-Gaussianity in the inflaton sector, e.g. [90, 119, 120, 132-136]. By measuring the precise momentum scaling of the squeezed bispectrum, one can in principle extract the masses and spins of any extra particles present during inflation. Since inflation excites all degrees of freedom with masses up to the inflationary Hubble scale (which may be as high as $10^{14} \mathrm{GeV}$ ), this potentially allows us to probe the particle spectrum far above the reach of terrestrial colliders.

Excited initial states By its very nature, inflation is very efficient at diluting initial inhomogeneities. Any traces of a pre-inflationary state in the CMB would either require inflation to have lasted not too much longer than required to solve the horizon problem, or to have the initial state contain excitations beyond the usual Bunch-Davies vacuum at arbitrarily short distances. This is a problematic proposition for various reasons (see e.g. [137, 138]). The effects of excited initial states were studied in an EFT analysis in $[139,140]$. The signal in the bispectrum peaks in the flattened momentum configuration $k_{1} \approx k_{2} \approx 2 k_{3}$. This is because the flattened configuration is sensitive to the presence of higher-derivative interactions that were more relevant at early times.

\subsubsection{Features}

The presence of any localized bumps or oscillatory features in the power spectrum or other correlation functions provides an interesting and powerful probe of deviations from the simplest realizations of single-field slow-roll inflation. A variety of physical processes can generate spectral features at any time during or after inflation (see [141] for a review). Most interesting for us are features that originated during inflation, as these can probe energy scales well beyond $H$ [142, 143] and may reveal new mass scales and interactions difficult to probe in any other way. The production of features during inflation may or may not involve violations of the slow-roll dynamics, or adiabaticity, and may or may not involve particle production.

Small amplitude periodic or localized features in the couplings of the EFT of inflation $\left(\epsilon_{1}(t)\right.$, $c_{\mathrm{S}}(t)$, etc.) lead to modulated oscillatory features superimposed on the almost scale-invariant

\footnotetext{
${ }^{7}$ This is expected, for instance from integrating out heavy degrees of freedom in a UV completion in which the background is protected by some internal symmetry softly broken by the inflationary potential if the inflaton is a pseudo-Goldstone boson with derivative interactions to these fields. This happens automatically in multi-scalar models if the group orbits are curved trajectories in the sigma model metric.
} 


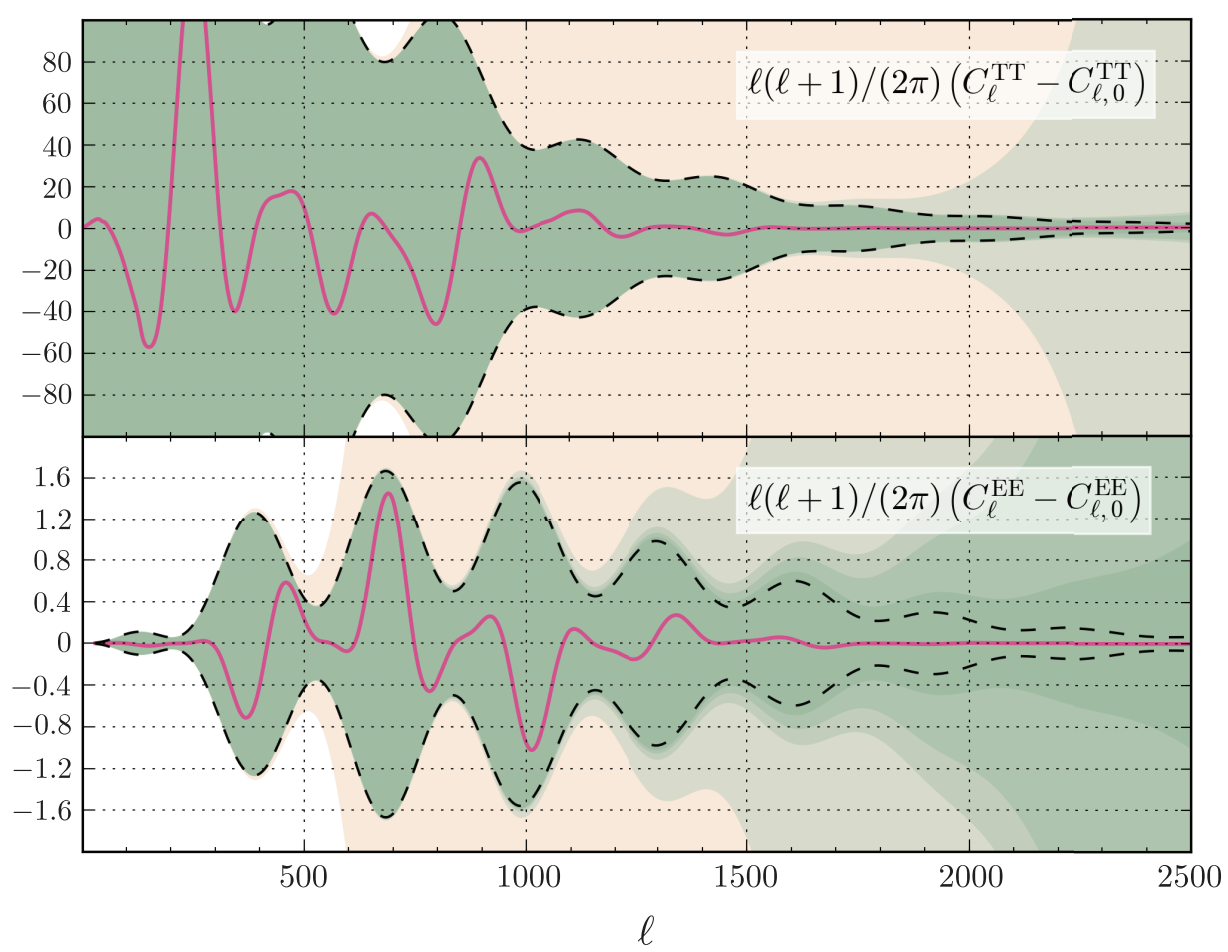

Figure 4: Example of a feature in the CMB power spectrum relative to the corresponding $c_{\mathrm{s}}=1$ featureless one $C_{\ell, 0}$ due to a transient reduction in the speed of sound $c_{\mathrm{s}}$ in single-field slow-roll inflation. CORE's approximated $68 \%$ error bars are represented in increasingly darker shades of green for the configurations in Tables 2 and 3 in Sec. 3. LiteBIRD's sensitivity corresponds to the orange-shaded region. The dashed line is the standard deviation due to cosmic variance. CORE's increased sensitivity in the polarization power spectrum is cosmic variance limited up to $\ell \approx 1500$. This power spectrum feature is accompanied by a correlated feature in the bispectrum, given in (2.8).

power spectrum, bispectrum, and higher order $n$-point functions. Because of their common origin, any such features are correlated [144] and the specific form of the correlations can be used to identify their origin and to improve their detectability $[145,146]$, in particular with joint searches in the power spectrum and bispectrum [147]. Periodic variations in $\epsilon_{1}(t)$ (for instance due to monodromy or small-scale structure in the scalar potential) can lead to a resonant enhancement of oscillatory features in the power spectrum or in the non-Gaussianity [148, 149]. Abrupt changes in $\epsilon_{1}$ and $c_{\mathrm{s}}$, typically associated with steps in the potential and other theoretically well motivated interruptions of slow roll (see e.g. [150] and other examples discussed in [52]), can also lead to oscillations and an enhancement of the bispectrum. In all cases it is important to check that the time and energy scales associated with the generation of the features are compatible with the use of the effective single-field or low energy description.

The superior constraining power of CORE is illustrated in Fig. 4 for the particular case of transient, moderate reductions in $c_{\mathrm{s}}$ away from $c_{\mathrm{s}}=1$ (a situation that is well-motivated theoretically and fully compatible with uninterrupted slow-roll and the single-field EFT description [145, 151]). These features look like enveloped linear oscillations in $k$, both in the primordial power spectrum and the bispectrum, and approximately so in the respective CMB projections, persisting over a 
relatively large range of scales. The power spectrum feature $\Delta \mathcal{P}_{\mathcal{R}} / \mathcal{P}_{\mathcal{R}}$ can be shown to be the Fourier transform of the reduction in $c_{\mathrm{s}}: \Delta \mathcal{P}_{\mathcal{R}} / \mathcal{P}_{\mathcal{R}}=k \int_{-\infty}^{0} \mathrm{~d} \tau\left(1-c_{\mathrm{s}}^{-2}\right) \sin (2 k \tau)$. The primordial bispectrum is given by

$$
\begin{aligned}
\Delta B_{\mathcal{R}}\left(k_{1}, k_{2}, k_{3}\right)=\frac{(2 \pi)^{4} \mathcal{P}_{\mathcal{R}}^{2}}{\left(k_{1} k_{2} k_{3}\right)^{2}}\left[c_{0}\left(k_{i}\right) \frac{\Delta \mathcal{P}_{\mathcal{R}}}{\mathcal{P}_{\mathcal{R}}}\left(\frac{k_{t}}{2}\right)\right. & +c_{1}\left(k_{i}\right) \frac{\mathrm{d}}{\mathrm{d} \log k_{t}} \frac{\Delta \mathcal{P}_{\mathcal{R}}}{\mathcal{P}_{\mathcal{R}}}\left(\frac{k_{t}}{2}\right) \\
& \left.+c_{2}\left(k_{i}\right)\left(\frac{\mathrm{d}}{\mathrm{d} \log k_{t}}\right)^{2} \frac{\Delta \mathcal{P}_{\mathcal{R}}}{\mathcal{P}_{\mathcal{R}}}\left(\frac{k_{t}}{2}\right)\right],
\end{aligned}
$$

where $k_{t} \equiv k_{1}+k_{2}+k_{3}$ and the coefficients $c_{i}$ are known functions [145] of $k_{1}, k_{2}, k_{3}$.

\subsubsection{Isocurvature}

The primordial seed perturbations for structure formation and CMB anisotropies could have been either pure curvature perturbations (the adiabatic mode), cold dark matter, baryon, or neutrino density isocurvature perturbations (i.e., spatial perturbations in the ratios of number densities of different particle species), neutrino velocity isocurvature perturbations or an arbitrarily correlated mixture of some or all of these [152, 153]. A detection of any type of isocurvature would be a smoking gun for multi-field inflationary models and would rule out single-field models. However, this is a one way implication. Lack of an isocurvature contribution to the CMB anisotropies does not rule out multi-field inflation, since even a large isocurvature contribution present immediately after inflation can be wiped out by later processes [154-156]. Planck has set tight upper bounds on the possible isocurvature contribution $[3,5]$. However, as we see in Section 7 , future CMB missions, in particular with CORE's sensitivity, may improve the constraints by a factor of 5 in simple one-parameter isocurvature extensions to the adiabatic $\Lambda$ CDM model. Conversely, this means that there is a relatively large window for an observation of a per thousand level isocurvature contribution. A CMB mission optimized for detecting or constraining the primordial tensor-toscalar ratio is also excellent for breaking the degeneracy between the large-scale isocurvature and tensor contributions. This mitigates the possibility of misinterpreting a nearly scale-invariant isocurvature contribution as a tensor contribution or vice versa. Finally, it is important to test how accurate and unbiased the determination of the standard cosmological parameters is if instead of the usually assumed purely adiabatic primordial mode, we allow for more general initial conditions of perturbations.

\subsection{Beginning and end of inflation}

\subsubsection{Spatial curvature}

False vacuum decay leads to pockets of space with negative spatial curvature [157]. The sign and the size of the curvature parameter $\Omega_{\mathrm{k}}$ are therefore interesting probes of the pre-inflationary state. Unfortunately, this will be hard to observe, given the efficiency with which the inflationary expansion dilutes pre-existing inhomogeneities including curvature. Values of $\left|\Omega_{\mathrm{k}}\right|>10^{-4}$ only survive if inflation did not last longer than the minimal duration required to solve the horizon problem [33]. 


\subsubsection{Topological defects}

Topological defects can form in symmetry-breaking phase transitions in the early Universe [158]. These transitions happen at the end of inflation in a class of models called hybrid inflation $[159,160]$. Alternatively, defects can form in phase transitions during inflation [161, 162] or afterwards [158, 163]. Searching for topological defects therefore yields information about the phase transition that formed them, the high-energy physics responsible for the phase transition, and ultimately can distinguish between models of inflation or even rule them out. The most commonly encountered defects in models of inflation-scale physics are cosmic strings (see [164, 165] for reviews).

Certain topological defects, including cosmic strings, produce a scale-invariant spectrum of density fluctuations and therefore are in principle compatible with the standard cosmology. By making precise measurements of the CMB, we can check how large an admixture of defects our Universe allows. The CMB temperature and polarization power spectra are proportional to the fourth power of the symmetry breaking scale, and so the CMB constraints on the fractional contribution of defects $f_{10}$ (by convention the fraction is taken at multipole $\ell=10$ in the temperature power spectrum) can be translated into constraints on that scale. As an example of the importance of searching for signals from defects, minimal D-term hybrid inflation is effectively ruled out because of its cosmic string contribution to the temperature power spectrum [166]. ${ }^{8}$

An important feature of the perturbations coming from topological defects is that they create a B-mode polarization signal. This signal would be in competition with that produced by primordial gravitational waves. In order to analyze the B-mode polarization signal correctly, the possibility that topological defects are the seed of the B-modes must be included. It may also be that gravitational waves and defects can both seed B-modes, and thus one has to be able to distinguish between them [167].

\section{Experimental configurations and forecasting methodology}

The purpose of this section is to explain the assumptions regarding the CORE instrumental capabilities for arriving at the forecasts presented in this paper. For a more detailed description and discussion of the CORE instrument, we refer the reader to the companion ECO mission paper [6], the ECO instrument paper [7] and the ECO systematics paper [8].

As part of the pre-proposal studies reported in the "Exploring Cosmic Origin with CORE" series, this paper mainly deals with the impact of the telescope size and noise sensitivity on the inflation science results. CORE will map the sky in temperature and polarization in 19 frequency channels spanning the $60-600 \mathrm{GHz}$ range with noise sensitivities and angular resolution summarized in Table 2. For our forecast we use the only the six channels in the frequency range $130-220 \mathrm{GHz}$ under the ideal assumption that foreground contaminations in these bands are completely removed by the lower and higher frequency channels and systematic effects are under control. In the following we will refer to this configuration as CORE-M5. This same assumption

\footnotetext{
${ }^{8}$ Hybrid inflation ends when a second "waterfall" field becomes unstable, triggering a symmetry-breaking phase transition. In $\mathcal{N}=1$ supergravity models, both the inflaton and the waterfall field are complex scalars (belonging to $\mathcal{N}=1$ chiral supermultiplets) and therefore the defects formed are cosmic strings. In the D-term case, the mass per unit length of the strings is fixed by the scale of inflation, and this is enough to rule out the minimal model.
} 


\begin{tabular}{|c|c|c|c|c|c|c|c|}
\hline $\begin{array}{c}\text { Channel } \\
\mathrm{GHz}\end{array}$ & $\begin{array}{l}\text { FWHM } \\
\text { [arcmin] }\end{array}$ & $N_{\text {det }}$ & $\begin{array}{c}\Delta T \\
{[\mu K \text { arcmin }]}\end{array}$ & $\begin{array}{c}\Delta P \\
{[\mu K \text { arcmin }]}\end{array}$ & $\begin{array}{c}\Delta I \\
{\left[\mu K_{\mathrm{RJ}} \operatorname{arcmin}\right]}\end{array}$ & $\begin{array}{c}\Delta I \\
{[\mathrm{kJy} / \mathrm{sr} \text { arcmin }]}\end{array}$ & $\begin{array}{c}\Delta y \times 10^{6} \\
{\left[y_{\mathrm{Sz}} \text { arcmin }\right]}\end{array}$ \\
\hline 60 & 17.87 & 48 & 7.5 & 10.6 & 6.81 & 0.75 & -1.5 \\
\hline 70 & 15.39 & 48 & 7.1 & 10 & 6.23 & 0.94 & -1.5 \\
\hline 80 & 13.52 & 48 & 6.8 & 9.6 & 5.76 & 1.13 & -1.5 \\
\hline 90 & 12.08 & 78 & 5.1 & 7.3 & 4.19 & 1.04 & -1.2 \\
\hline 100 & 10.92 & 78 & 5.0 & 7.1 & 3.90 & 1.2 & -1.2 \\
\hline 115 & 9.56 & 76 & 5.0 & 7.0 & 3.58 & 1.45 & -1.3 \\
\hline 130 & 8.51 & 124 & 3.9 & 5.5 & 2.55 & 1.32 & -1.2 \\
\hline 145 & 7.68 & 144 & 3.6 & 5.1 & 2.16 & 1.39 & -1.3 \\
\hline 160 & 7.01 & 144 & 3.7 & 5.2 & 1.98 & 1.55 & -1.6 \\
\hline 175 & 6.45 & 160 & 3.6 & 5.1 & 1.72 & 1.62 & -2.1 \\
\hline 195 & 5.84 & 192 & 3.5 & 4.9 & 1.41 & 1.65 & -3.8 \\
\hline 220 & 5.23 & 192 & 3.8 & 5.4 & 1.24 & 1.85 & - \\
\hline 255 & 4.57 & 128 & 5.6 & 7.9 & 1.30 & 2.59 & 3.5 \\
\hline 295 & 3.99 & 128 & 7.4 & 10.5 & 1.12 & 3.01 & 2.2 \\
\hline 340 & 3.49 & 128 & 11.1 & 15.7 & 1.01 & 3.57 & 2.0 \\
\hline 390 & 3.06 & 96 & 22.0 & 31.1 & 1.08 & 5.05 & 2.8 \\
\hline 450 & 2.65 & 96 & 45.9 & 64.9 & 1.04 & 6.48 & 4.3 \\
\hline 520 & 2.29 & 96 & 116.6 & 164.8 & 1.03 & 8.56 & 8.3 \\
\hline 600 & 1.98 & 96 & 358.3 & 506.7 & 1.03 & 11.4 & 20.0 \\
\hline Array & & 2100 & 1.2 & 1.7 & & & 0.41 \\
\hline
\end{tabular}

Table 2: CORE-M5 proposed frequency channels. Sensitivities are calculated assuming $\Delta \nu / \nu=$ $30 \%$ bandwidth, $60 \%$ optical efficiency, a total noise twice the expected photon noise from the sky, and the optics of the instrument at $40 \mathrm{~K}$ temperature. This configuration has 2100 detectors, of which about $45 \%$ lie in the CMB channels between 130 and $220 \mathrm{GHz}$. The six CMB channels yield an aggregated CMB sensitivity of $2 \mu \mathrm{K} \cdot \operatorname{arcmin}(1.7 \mu \mathrm{K} \cdot \operatorname{arcmin}$ for the full array).

that lower and higher frequencies suffice to remove completely foreground contamination in the inverse noise weighted combination of the six central frequency channels is used in the companion ECO paper on cosmological parameters. A dedicated paper of this ECO series [10] studies in great detail the capability to measure primordial B-mode polarization by a component separation approach that makes use of all the frequency channels and takes into account the foreground contamination of anomalous microwave emission, thermal dust emission, synchrotron, and point sources. Updating all the science forecast for cosmology with the inclusion of the main results of this component separation dedicated study [10] is left for a future work, but we will however refer to the impact of foreground residuals as estimated in [168].

In this paper, we compare our forecasts for CORE-M5 to those obtained with experimental configurations from other concepts for the next space missions dedicated to CMB polarization, such as (a) the LiteBIRD-ext configuration [168] for JAXA LiteBIRD [27], (b) three configurations with the same noise sensitivity in $\mu K$-arcmin as LiteBIRD-ext, but with higher angular resolution thanks to a larger telescope of $80 \mathrm{~cm}$ (LiteCORE-80) or $120 \mathrm{~cm}$ (LiteCORE-120) or $150 \mathrm{~cm}$ (LiteCORE-150), and (c) the COrE + proposal previously submitted to ESA in response to the M4 mission call and its version optCOrE + with an extended mission duration. Note that we will refer to the LiteCORE-80 forecasts as those representative for a possible downscoped version 
of CORE-M5, called MiniCORE [7], with a $80 \mathrm{~cm}$ telescope and covering the reduced 100-600 $\mathrm{GHz}$ frequency. For all these configurations, we consider an inverse noise weighted combination of central frequency channels, always under the assumption that lower and higher frequency channels remove completely the foreground contamination. Table 3 reports the experimental specifications for the central frequency channels of these configurations. This comparison sheds light on the role that angular resolution and noise detector sensitivity plays in determining what new conclusions concerning inflation can be extracted from the data of a particular concept for a future CMB space mission. See also [169-171] for previous forecasts for inflation science comparing different future experiments dedicated to CMB polarization.

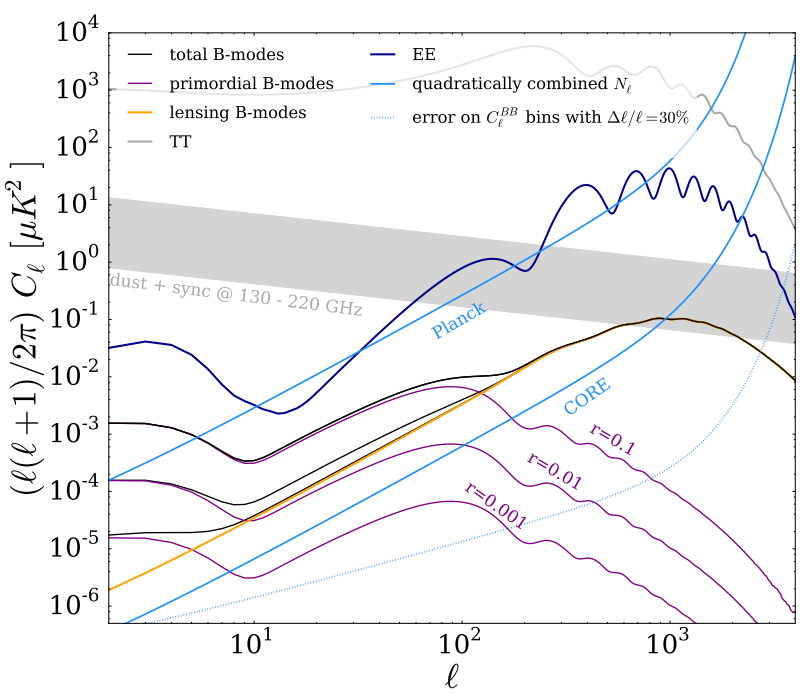

Figure 5: Comparison of cosmological signals, in particular the primordial and lensed $B$-modes, with diffuse astrophysical foregrounds amplitudes. The noise levels for Planck and CORE are also displayed.
This comparison is important since the cost of a CMB polarization space mission is largely determined by the size of the instrument, or equivalently the size of the primary aperture. Because the resolution is diffraction limited, the angular size is inversely proportional to the effective mirror diameter. LiteBIRD envisages a smaller mirror size and thus would have a coarser angular resolution. On the one hand, detecting primordial $\mathrm{B}$ modes from inflation does not require necessarily an exquisite angular resolution. There are the two windows for detecting such B modes: the so-called "reionization" bump, situated on very large angular scales not accessible from the ground (i.e., $\ell \lesssim 10$ ); and the so-called "recombination" bump,

for which the statistically exploitable information is centered around $\ell \approx 80$. [See Fig. 5.] Above $\ell \gtrsim 100$, beyond approximately where the modes had already entered the horizon at last scattering, the primordial $B$ mode signal plummets while the contaminant signal from gravitational lensing continues to rise. Given the current constraints on $r$ [172], there is not much exploitable information regarding the primordial B modes on scales with $\ell \gtrsim 100$.

However there are two reasons other than ancillary science why an angular resolution extending well beyond $\ell \approx 100$ is highly desirable. Firstly, when $r \lesssim 10^{-2}$, the gravitational lensing signal starts to exceed any possible primordial $B$ mode signal beyond the reionization bump. If this gravitational lensing $B$ mode cannot be removed, it becomes the dominant source of noise when the detector noise level becomes smaller than $\approx 5 \mu K \cdot \operatorname{arcmin}$. Two approaches to dealing with this parasitic lensing are possible. (1) One can try to characterize the power spectrum of this lensing contaminant at exquisite precision. In this case $C_{\ell}^{B B}$ can be measured after broad binning (i.e., with $\Delta \ell / \ell \approx 1$ ) to an accuracy of approximately $\tilde{N}_{\ell}^{B B} / \ell$ in the broad bins, where here $\tilde{N}_{\ell}^{B B}$ 
includes both the gravitational lensing and the detector noise contributions. The success of this

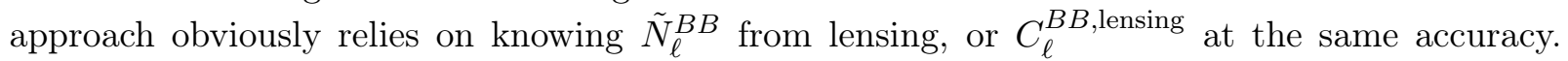
(2) Another more ambitious approach is what is called "de-lensing." Delensing is predicting

$a_{\ell m}^{B \text {, lensing }}$ based on $a_{\ell m}^{E}$ and $a_{\ell m}^{\phi \text {,lensing }}$ where $\phi$ is the CMB lensing potential and subtracting this prediction from the measured B mode map. $a_{\ell m}^{B \text {,lensing }}$ at low $\ell$ of interest for detecting primordial B mode signal may be though of as the low $\ell$ "white noise" tail of many small, weakly-correlated gravitationally lensed regions. Mathematically, $a_{\ell m}^{B, l e n s i n g}$ may be expressed as a sort of convolution of $a_{\ell m}^{E}$ and $a_{\ell m}^{\phi, l e n s i n g}$, and the multipole numbers making the dominant contribution have $\ell \approx$ $O\left(10^{3}\right)$. This is why both exquisite angular resolution and sensitivity are needed to be able to "de-lens."

\subsection{Simplified likelihood for forecasts}

Assuming that CMB anisotropies are Gaussian and statistically isotropic, we use the following $\chi_{\text {eff }}^{2}$ for our science forecasts $[56,173]$ :

$$
\chi_{\text {eff }}^{2}=-2 \ln \mathcal{L}=\sum_{\ell}(2 \ell+1) f_{\text {sky }}\left\{\operatorname{Tr}\left[\hat{\mathbf{C}}_{\ell} \overline{\mathbf{C}}_{\ell}^{-1}\right]+\ln \left|\hat{\mathbf{C}}_{\ell} \overline{\mathbf{C}}_{\ell}^{-1}\right|-n\right\},
$$

where $\overline{\mathbf{C}}_{\ell}$ and $\hat{\mathbf{C}}_{\ell}$ denote the theoretical and observed data covariance matrices, respectively, and $n$ is a normalization factor. The covariance matrix depends on the power spectra $C_{\ell}^{X Y}$ where $X$ and $Y$ can take the values $T, E$ (with $n=2$ ), T, E, B or $T, E, P$ (with $n=3$ ), or T, E, B, $P$ (with $n=4$ ), where $P$ denotes lensing potential. In the most general case the theoretical covariance matrix is

$$
\overline{\boldsymbol{C}}_{\ell} \equiv\left[\begin{array}{cccc}
\bar{C}_{\ell}^{T T}+N_{\ell}^{T T} & \bar{C}_{\ell}^{T E} & 0 & \bar{C}_{\ell}^{T P} \\
\bar{C}_{\ell}^{T E} & \bar{C}_{\ell}^{E E}+N_{\ell}^{E E} & 0 & \bar{C}_{\ell}^{E P} \\
0 & 0 & \bar{C}_{\ell}^{B B}+N_{\ell}^{B B} & 0 \\
\bar{C}_{\ell}^{T P} & \bar{C}_{\ell}^{E P} & 0 & \bar{C}_{\ell}^{P P}+N_{\ell}^{P P}
\end{array}\right]
$$

where $N_{\ell}^{\mathrm{XX}}$ are the noise power spectra. These account for the instrumental noise $N_{\ell}^{\mathrm{XX} \text {,inst }}$; when $X=B$ and internal delensing is possible, the residual delensing error can also be included. For $X=T, E, B$, for each experimental configuration studied, we consider an inverse-variance weighted sum of the noise sensitivity convolved with a Gaussian beam window function for each frequency channel $\nu$ :

$$
N_{\ell}^{\mathrm{XX} \text {,inst }}=\left[\sum_{\nu} \frac{1}{N_{\ell \nu}^{\mathrm{XX}, \text { inst }}}\right]^{-1}
$$

with

$$
N_{\ell \nu}^{\mathrm{X} X, \text { inst }}=w_{X \nu}^{-1} \exp \left[\ell(\ell+1) \frac{\theta_{\mathrm{FWHM} \nu}}{8 \ln 2}\right] .
$$

Here $w_{\mathrm{E} \nu}^{-1 / 2}=w_{\mathrm{B} \nu}^{-1 / 2}\left(=w_{\mathrm{T} \nu}^{-1 / 2} \sqrt{2}\right)$ is the detector noise level on a steradian patch for polarization (temperature) and $\theta_{\mathrm{FWHM}}$ being the full width half maximum (FWHM) of the beam in radians for a given frequency channel $\nu$. In Eq. (3.1) we consider $\ell_{\max }=1350$ for LiteBIRD, $\ell_{\max }=2400$ for LiteCORE-80 and $\ell_{\max }=3000$ for all the other CORE configurations in Table 2 and 3 . We 


\begin{tabular}{|c|c|c|c|}
\hline Channel [GHz] & FWHM [arcmin] & $\Delta T[\mu \mathrm{K}$ arcmin $]$ & $\Delta P[\mu \mathrm{K}$ arcmin $]$ \\
\hline \multicolumn{4}{|c|}{ LiteBIRD, $f_{\text {sky }}=0.7$} \\
\hline 78 & 55 & 8.8 & 12.5 \\
\hline 88.5 & 49 & 7.1 & 10.0 \\
\hline 100 & 43 & 8.5 & 12.0 \\
\hline 118.9 & 36 & 6.7 & 9.5 \\
\hline 140 & 31 & 5.3 & 7.5 \\
\hline 166 & 26 & 5.0 & 7.0 \\
\hline 195 & 22 & 3.6 & 5.0 \\
\hline \multicolumn{4}{|c|}{ LiteCORE-80, $f_{\text {sky }}=0.7$} \\
\hline 80 & 20.2 & 8.8 & 12.5 \\
\hline 90 & 17.8 & 7.1 & 10.0 \\
\hline 100 & 15.8 & 8.5 & 12.0 \\
\hline 120 & 13.2 & 6.7 & 9.5 \\
\hline 140 & 11.2 & 5.3 & 7.5 \\
\hline 166 & 8.5 & 5.0 & 7.0 \\
\hline 195 & 8.1 & 3.6 & 5.0 \\
\hline \multicolumn{4}{|c|}{ LiteCORE-120, $f_{\text {sky }}=0.7$} \\
\hline 80 & 13.5 & 8.8 & 12.5 \\
\hline 90 & 11.9 & 7.1 & 10.0 \\
\hline 100 & 10.5 & 8.5 & 12.0 \\
\hline 120 & 8.8 & 6.7 & 9.5 \\
\hline 140 & 7.4 & 5.3 & 7.5 \\
\hline 166 & 6.3 & 5.0 & 7.0 \\
\hline 195 & 5.4 & 3.6 & 5.0 \\
\hline \multicolumn{4}{|c|}{ LiteCORE-150, $f_{\text {sky }}=0.7$} \\
\hline 80 & 10.8 & 8.8 & 12.5 \\
\hline 90 & 9.5 & 7.1 & 10.0 \\
\hline 100 & 8.4 & 8.5 & 12.0 \\
\hline 120 & 7.0 & 6.7 & 9.5 \\
\hline 140 & 5.9 & 5.3 & 7.5 \\
\hline 166 & 5.0 & 5.0 & 7.0 \\
\hline 195 & 4.3 & 3.6 & 5.0 \\
\hline \multicolumn{4}{|c|}{ (opt) $\mathrm{COrE}+, f_{\mathrm{sky}}=0.7$} \\
\hline 100 & 8.4 & $6.0(4.2)$ & $8.5(6.0)$ \\
\hline 115 & 7.3 & $5.0(3.5)$ & $7.0(5.0)$ \\
\hline 130 & 6.5 & $4.2(3.0)$ & $5.9(4.2))$ \\
\hline 145 & 5.8 & $3.6(2.5)$ & $5.0(3.6)$ \\
\hline 160 & 5.3 & $3.8(2.7)$ & $5.4(3.8)$ \\
\hline 175 & 4.8 & $3.8(2.7)$ & $5.3(3.8)$ \\
\hline 195 & 4.3 & $3.8(2.7)$ & $5.3(3.8)$ \\
\hline 220 & 3.8 & $5.8(4.1)$ & $8.1(5.8)$ \\
\hline
\end{tabular}

Table 3: Experimental specifications for LiteBIRD-ext, LiteCORE-80, LiteCORE-120, LiteCORE-150, and (opt) $\mathrm{COrE}+$.

assume here that beam and other systematic uncertainties are much smaller than the statistical errors.

In addition to the primary CMB temperature and polarization anisotropies, we consider the cosmological information contained in the CMB lensing potential power spectrum $C_{\ell}^{P P}$. Most 
of the experimental configurations studied in this paper allow a precise reconstruction of the CMB lensing potential. It is well known that $C_{\ell}^{P P}$ helps to break parameter degeneracies and to determine the absolute neutrino masses [174]. We therefore fold in the CMB lensing information in our likelihood [corresponding to $n=3$ case when considering $T, E, P$ or the most general case $n=4$ in Eq. (3.2)] by neglecting the temperature/polarization-lensing cross-correlation $\left(C_{\ell}^{T P}, C_{\ell}^{E P}\right)$ and considering lensed spectra $C_{\ell}^{T T}, C_{\ell}^{T E}, C_{\ell}^{E E}$, as explained in the ECO paper on cosmological parameters [9].

We use the publicly available Einstein-Boltzmann codes CAMB [175] to compute the theoretical predictions for temperature, polarization, and gravitational lensing deflection power spectra. We use Recfast to model the cosmic recombination history in the forecasts. For future data analyses, the differences with the detailed cosmological recombination codes CosmoRec [176] and HyReci [177] can be avoided but have shown to be small $\left(\approx 0.3 \sigma\right.$ for $\left.n_{\mathrm{S}}\right)$ even at the level of precision that could be reached with CORE [9]. Unless otherwise specified, we consider for our mock data a fiducial $\Lambda$ CDM cosmology compatible with Planck constraints [178]. We assume a flat Universe with a cosmological constant and three massless neutrinos, $\Omega_{\mathrm{b}} h^{2}=0.02214$ and $\Omega_{\mathrm{c}} h^{2}=0.1206$ as the baryon and cold dark matter physical densities, respectively. We choose the optical depth to reionization $\tau=0.0581$, the Hubble parameter $H_{0}=66.89 \mathrm{~km} \mathrm{~s}^{-1} \mathrm{Mpc}^{-1}, A_{\mathrm{s}}=2.1179 \times 10^{-9}$ and spectral index $n_{\mathrm{s}}=0.9625$ as amplitude and tilt for the spectrum of primordial perturbations, respectively. We generate posterior probability distributions for the parameters using either the Metropolis-Hastings algorithm implemented in CosmoMC [179], the nested sampling algorithm MultiNest [180-182], or PolyChord, which combines nested sampling with slice sampling [183].

\subsection{Dealing with gravitational lensing}

In the search to detect primordial gravitational waves, CMB lensing can be considered as equivalent to an additional noise source whose power spectrum in turn depends on a number of other non-inflationary cosmological parameters. Gravitational lensing mixes the $E$ and $B$ polarization modes [184]. The amplitude of these lensing $B$-modes peaks at around a multipole of $\ell \approx 1000$ and is always larger than the primordial signal on scales smaller than the reionization bump for the current upper bounds on $r$ [172]. Lensing is therefore, after the diffuse astrophysical foregrounds, the second largest contaminant in the search for $r$, cf. Fig. 5.

Techniques have been proposed to delens the CMB polarization maps [185-191] - that is, to reduce the extra variance induced by lensing. Delensing proposes to subtract the lensing induced $\mathrm{B}$ mode from the observed CMB B mode map, which requires estimating the unlensed CMB as well as the matter distribution $P$ (in the form of a lensing potential). The performance of delensing depends on the experimental characteristics and on the choice for the matter distribution estimator (for a detailed analysis of the delensing efficiency and its propagation to limits on $r$ see [168]). In this paper, we consider internal CMB delensing, in order to investigate the performance of each experimental configuration alone without the help of external data. Given its limited internal delensing capabilities, we consider the full signal in B-mode polarization (primordial plus lensing) for LiteBIRD, whereas we consider the full delensed option for the various CORE configurations. As an estimate for the noise spectrum $N_{\ell}^{P P}$ of the CMB lensing potential, we consider the $E B$ estimator [192] as in [168]. 


\section{Probing inflationary parameters with CORE}

In this Section we begin the presentation of our science forecasts dedicated to the physics of inflation for the CMB space mission configurations previously described. Different priors can be used to compare inflationary theoretical predictions to CMB anisotropies power spectra. In this Section we employ the physical parameterization of the primordial power spectra of scalar and tensor perturbations, extending Eq. (1.1) as:

$$
\begin{aligned}
& \mathcal{P}_{\mathcal{R}}(k)=A_{\mathrm{S}}\left(\frac{k}{k_{*}}\right)^{n_{\mathrm{s}}-1+\frac{1}{2} \frac{\mathrm{d} n_{\mathrm{s}}}{\mathrm{d} \ln k} \ln \left(k / k_{*}\right)+\ldots} \\
& \mathcal{P}_{\mathrm{t}}(k)=r A_{\mathrm{S}}\left(\frac{k}{k_{*}}\right)^{n_{\mathrm{t}}+\frac{1}{2} \frac{\mathrm{d} n_{\mathrm{t}}}{\mathrm{d} \ln k} \ln \left(k / k_{*}\right)+\ldots} .
\end{aligned}
$$

Physical parameters such as $A_{\mathrm{s}}, n_{\mathrm{s}}, \mathrm{d} n_{\mathrm{s}} / \mathrm{d} \ln k, r, n_{\mathrm{t}}$ will be considered as the primary parameters, together with $\omega_{\mathrm{b}}, \omega_{\mathrm{c}}, \tau$ and $\theta_{\mathrm{MC}}$ (the latter being the CosmoMC variable for the angular size of sound horizon, i.e., $\left.r_{*} / D_{A}\right)$. In the next Section the dependence of the slow roll predictions for the primordial power spectra on the Hubble flow functions (HFFs henceforth) will be investigated and the HFFs will be sampled as the primary parameters. The consistent results obtained by these two approaches have been studied in [3, 193, 194]. In the next Subsections we will discuss the improvements on the measurement of $n_{\mathrm{s}}$ and its scale dependence, the expected constraints on the tensor-to-scalar ratio and the tensor tilt, and finally the forecasts for the constraints on the spatial curvature.

\subsection{Forecasts for the spectral index and its scale dependence}

One of the main Planck results has been to provide an accurate measurement of $n_{\mathrm{s}}<1$ that rules out the Harrison-Zeldovich scale invariant primordial power spectrum at more than $5 \sigma[3,5]$. This measurement suggests a preference for models with a natural exit from inflation and ruled out hybrid inflationary models predicting $n_{\mathrm{s}}>1$.

\begin{tabular}{|c|c|c|c|c|}
\hline Parameter & LiteBIRD, TE & LiteCORE-80, TEP & CORE-M5, TEP & COrE,+ TEP \\
\hline$\Omega_{\mathrm{b}} h^{2}$ & $0.02214 \pm 0.00013$ & $0.022140 \pm 0.000052$ & $0.022141 \pm 0.000037$ & $0.022141 \pm 0.000033$ \\
$\Omega_{\mathrm{c}} h^{2}$ & $0.12059 \pm 0.00099$ & $0.12058 \pm 0.00033$ & $0.1205857 \pm 0.00027$ & $0.12058 \pm 0.00025$ \\
$100 \theta_{\mathrm{MC}}$ & $1.03922 \pm 0.00030$ & $1.039220 \pm 0.000099$ & $1.039223 \pm 0.000077$ & $1.039224 \pm 0.000073$ \\
$\tau$ & $0.0582 \pm 0.0021$ & $0.0582 \pm 0.0020$ & $0.0583 \pm 0.0020$ & $0.0582 \pm 0.0020$ \\
$n_{\mathrm{s}}$ & $0.9625 \pm 0.0034$ & $0.9625 \pm 0.0016$ & $0.9625 \pm 0.0014$ & $0.9626 \pm 0.0014$ \\
$\ln \left(10^{10} A_{\mathrm{s}}\right)$ & $3.0533 \pm 0.0045$ & $3.0533 \pm 0.0038$ & $3.0533 \pm 0.0035$ & $3.0533 \pm 0.0034$ \\
\hline$H_{0}$ & $66.89 \pm 0.47$ & $66.89 \pm 0.14$ & $66.89 \pm 0.11$ & $66.90 \pm 0.10$ \\
$\sigma_{8}$ & $0.8285 \pm 0.0039$ & $0.8285 \pm 0.0013$ & $0.8285 \pm 0.0011$ & $0.8285 \pm 0.0010$ \\
\hline
\end{tabular}

Table 4: Forecast 68\% CL constraints on primary and derived cosmological parameters for LiteBIRD, LiteCORE-80, CORE-M5 and COrE+configurations for the $\Lambda$ CDM model.

Table 4 reports the forecast $68 \%$ CL uncertainties on the cosmological parameters of the $\Lambda$ CDM model obtained by LiteBIRD, LiteCORE-80, CORE-M5, and COrE + . Note that LiteCORE-80, CORE-M5 and COrE + results include lensing in the mock likelihood. All four configurations 
will provide a nearly cosmic-variance limited measurement of the $E E$ power spectrum at low multipoles and will therefore lead to a determination of the optical depth with an uncertainty close to the ideal case. Because of a better noise sensitivity and angular resolution, CORE-M5 results are better than those achievable by LiteCORE- 80 . The COrE + configuration with a $1.5 \mathrm{~m}$ class telescope, leads to further $11 \%, 6 \%, 4 \%$, and $3 \%$ improvements in the determination of $\Omega_{\mathrm{b}} h^{2}$, $\Omega_{\mathrm{c}} h^{2}, \theta_{\mathrm{MC}}$, and $\ln \left(10^{10} A_{\mathrm{s}}\right)$, respectively, with the uncertainties in the other primary parameters unchanged.

CORE-M5 tightens the uncertainties of the primary cosmological parameters with respect to the Planck 2015 results including high- $\ell$ polarization by factors between 4 and 10, and between 3 and 5 with respect to those including the most recent determination of the reionization optical depth [178]. The LiteBIRD improvement on the Planck results is rather different: there is a significant reduction in uncertainties on $\tau, \ln \left(10^{10} A_{\mathrm{s}}\right)$. But no gain on parameters such as $\theta_{\mathrm{MC}}$ [178] is expected due to the coarser angular resolution. It is interesting to note that a larger reduction of uncertainty with respect to most recent Planck results occurs on derived parameters such as the Hubble constant $H_{0}$. Given the current tension between CMB and the most recent local measurements of $H_{0}$ [46], CORE-M5 will help to clarify whether new physics beyond the $\Lambda \mathrm{CDM}$ model is required. See [9] for an analogous discussion of the larger expected improvement on the uncertainty on $\sigma_{8}$, whose value obtained from the CMB currently disagrees by about $2 \sigma$ with the measurement obtained using galaxy shear measurements [49, 50].

CORE-M5 will achieve an accuracy in the determination of $n_{\mathrm{s}}$ more than three times better than the current Planck uncertainty. The CORE-M5 error on $n_{\mathrm{s}}$ will be comparable to the standard theoretical uncertainty arising from the entropy generation stage after inflation [3], which enters into the comparison of CMB data with specific inflationary models. The predictions for a given inflationary model will be sensitive not only to the form of the potential during inflation but also to the subsequent reheating stage during which the inflaton decays, leading to a fully thermalized Universe. In the next Section this aspect will be explored quantitatively for the inflationary models studied in Ref. [102].

Inflation as modelled by a slowly rolling scalar field generically predicts a small running of the spectral index of the primordial spectra [195], which in terms of the slow-roll parameters is of higher order than the deviation of the scalar spectral index from unity. Although a theoretical prior based on the slow roll approximation makes the running of the scalar spectral index undetectably small for the precision of cosmological observations achieved so far [196, 197], its value inferred by CMB data was not merely a consistency check. Negative values of the running could be easily accommodated by pre-Planck data since the WMAP first year data release. See for example the WMAP 7 year data combined with SPT result $\mathrm{d} n_{\mathrm{s}} / \mathrm{d} \ln k=-0.024 \pm 0.011$ [198]. With the most precise measurement of the $\mathrm{CMB}$ anisotropies in the region of the higher acoustic peaks, the Planck data are compatible with a vanishing running of the spectral index. See the Planck 2015 temperature and polarization (TT,TE,EE + lowP) constraint $\mathrm{d} n_{\mathrm{s}} / \mathrm{d} \ln k=-0.006 \pm 0.007$ at $68 \% \mathrm{CL}$ [5] or the most recent result $\mathrm{d} n_{\mathrm{s}} / \mathrm{d} \ln k=-0.003 \pm 0.007$ at $68 \%$ CL, obtained with the latest measurement of the reionization optical depth $\tau$ [178].

Table 5 reports the forecasts for the cosmological parameters when $\mathrm{d} n_{\mathrm{s}} / \mathrm{d} \ln k$ is allowed to vary in addition to the parameters of the standard $\Lambda$ CDM model for a fiducial cosmology in which $\mathrm{d} n_{\mathrm{s}} / \mathrm{d} \ln k=0$. As indicated in Table 8 , the LiteBIRD forecast error on the running does not 


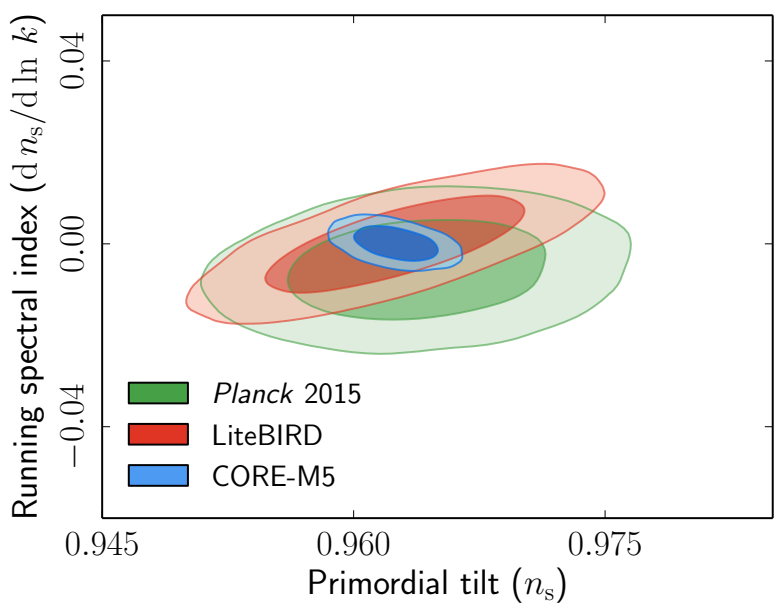

Figure 6: Forecast $68 \%$ and $95 \%$ CL $2 \mathrm{D}$ marginalized regions for $\left(n_{\mathrm{s}}, d n_{\mathrm{s}} / d \ln k\right)$ for CORE-M5 (blue) and LiteBIRD (red). These forecasts assume $d n_{\mathrm{s}} / d \ln k=0$ as the fiducial value. The green contours showing the $68 \%$ and 95\% CL for Planck 2015 TT + lowP [5] are displayed for comparison. Note that the Planck 2015 marginalized regions are based on real data whose best fit is different from the fiducial cosmology used in this paper.

improve significantly on the current Planck constraints. Moreover, the LiteBIRD uncertainty on the spectral index increases by $44 \%$ when the running is allowed to vary. By contrast, CORE-M5 will be able to reduce the current uncertainty by approximatively a factor of 3 . The forecast error on the spectral index increases by only $7 \%$ when the running is allowed to vary. COrE + would only marginally improve on the CORE-M5 forecast uncertainties for the running.

It is now understood that the negative running spectral index allowed by the pre-Planck data was driven by the low amplitude of the temperature power spectrum at $\ell \lesssim 40$ for the WMAP data. The relatively larger impact of this anomaly at $\ell \lesssim 40$ for WMAP data could have then conspired through calibration uncertainties in the combination with complementary CMB data at higher angular resolution such as SPT to accommodate a larger negative running. Thanks to the measurement of $C_{\ell}$ from $\ell=2$ to 2500 , Planck was able to show that the poor fit obtained by the running to the low- $\ell$ anomaly at $\ell \lesssim 40$ can be improved by other templates for deviations from a power law primordial power spectrum on large scales, although not at a significant statistical level [5]. As an example of such extensions, we consider a scale dependence of the running (i.e., a running of the running $\mathrm{d}^{2} n_{\mathrm{s}} / \mathrm{d} \ln k^{2}$ ), which was already discussed in Section 2. Although there is no statistical evidence of a scale dependence of $n_{\mathrm{s}}$ to second order, a combination of positive running and positive running of the running with a lower value of the spectral index at $k_{*}=0.05$ $\mathrm{Mpc}^{-1}$ are allowed by Planck 2015 data, e.g. $n_{\mathrm{s}}=0.9586 \pm 0.0056, \mathrm{~d} n_{\mathrm{s}} / \mathrm{d} \ln k=0.09 \pm 0.010$, $\mathrm{d}^{2} n_{\mathrm{s}} / \mathrm{d} \ln k^{2}=0.025 \pm 0.013$ at $68 \%$ CL with Planck TT,TE,EE + lowP, leading to a $\Delta \chi^{2} \sim-5$ improvement over the $\Lambda \mathrm{CDM}$ [5]. A conservative reconstruction of the inflationary potential including a fourth derivative of the inflaton potential beyond the slow-roll approximation supports similar considerations [5]. Table 6 reports the forecast uncertainties of the cosmological parameters when the running and the running of the running are allowed to vary around a fiducial cosmology with $\mathrm{d} n_{\mathrm{s}} / \mathrm{d} \ln k=\mathrm{d}^{2} n_{\mathrm{s}} / \mathrm{d} \ln k^{2}=0$. We obtain $\sigma\left(\mathrm{d} n_{\mathrm{s}} / \mathrm{d} \ln k\right) \approx 0.0024(0.0023)$ and $\sigma\left(\mathrm{d}^{2} n_{\mathrm{s}} / \mathrm{d} \ln k^{2}\right) \approx 0.0046(0.0043)$ as CORE-M5 (COrE +$)$ uncertainties on the running and on the 

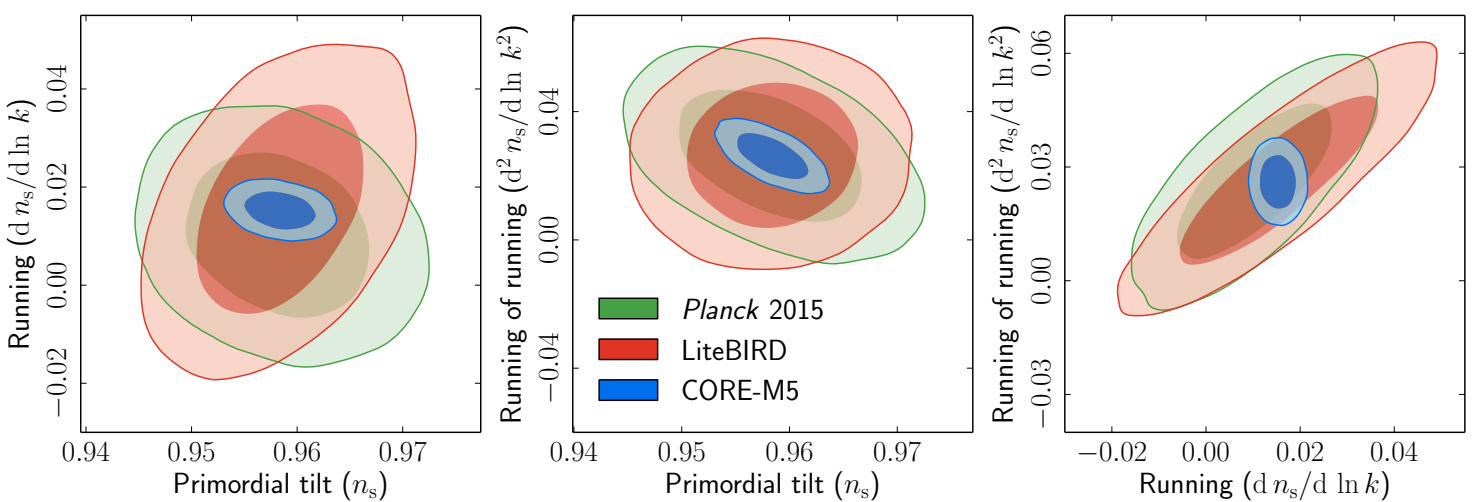

Figure 7: Forecast $68 \%$ and $95 \%$ CL $2 \mathrm{D}$ marginalized regions for $\left(n_{\mathrm{s}}, \mathrm{d} n_{\mathrm{s}} / \mathrm{d} \ln k\right)$ (left panel), $\left(n_{\mathrm{s}}, \mathrm{d}^{2} n_{\mathrm{s}} / \mathrm{d} \ln k^{2}\right)$ (middle panel) and $\left(\mathrm{d} n_{\mathrm{s}} / \mathrm{d} \ln k, \mathrm{~d}^{2} n_{\mathrm{s}} / \mathrm{d} \ln k^{2}\right)$ (right panel) for CORE-M5 (blue) and LiteBIRD (red). These forecasts assume as the fiducial values the Planck 2015 best fits including the running of the running [5]. The green contours showing the $68 \%$ and $95 \%$ CL for Planck 2015 TT,TE,EE + lowP are displayed for comparison.

running of the running, respectively. The CORE-M5 forecast uncertainties improve approximatively by a factor of 5 and 3 with respect to LiteBIRD for $\mathrm{d} n_{\mathrm{s}} / \mathrm{d} \ln k$ and $\mathrm{d}^{2} n_{\mathrm{s}} / \mathrm{d} \ln k^{2}$, respectively. Note that LiteBIRD does not significantly improve on the Planck constraints. There is a trade off between the better LiteBIRD noise sensitivity leading to a nearly cosmic variance limited measurement of the E-mode polarization at low and intermediate multipoles and the Planck higher resolution. This trade-off between LiteBIRD and Planck will be evident also in the different analysis presented in the next Sections.

\begin{tabular}{|c|c|c|c|c|}
\hline Parameter & LiteBIRD, TE & LiteCORE- 80, TEP & CORE-M5, TEP & COrE,+ TEP \\
\hline$\Omega_{\mathrm{b}} h^{2}$ & $0.02214 \pm 0.00013$ & $0.022140 \pm 0.000059$ & $0.022139 \pm 0.000044$ & $0.022140 \pm 0.000038$ \\
$\Omega_{\mathrm{c}} h^{2}$ & $0.1206 \pm 0.0011$ & $0.12058 \pm 0.00034$ & $0.12059 \pm 0.00028$ & $0.12059 \pm 0.00027$ \\
$100 \theta_{\mathrm{MC}}$ & $1.03922 \pm 0.00030$ & $1.039225 \pm 0.000099$ & $1.039225 \pm 0.000078$ & $1.039223 \pm 0.000072$ \\
$\tau$ & $0.0583 \pm 0.0021$ & $0.0583 \pm 0.0021$ & $0.0582 \pm 0.0020$ & $0.0582 \pm 0.0019$ \\
$n_{\mathrm{s}}$ & $0.9625 \pm 0.0049$ & $0.9625 \pm 0.0017$ & $0.9625 \pm 0.0014$ & $0.9626 \pm 0.0016$ \\
$d n_{\mathrm{s}} / d \ln k$ & $0.0000 \pm 0.0067$ & $0.0000 \pm 0.0030$ & $0.0000 \pm 0.0024$ & $0.0000 \pm 0.0023$ \\
$\ln \left(10^{10} A_{\mathrm{s}}\right)$ & $3.0534 \pm 0.0055$ & $3.0531 \pm 0.0038$ & $3.0532 \pm 0.0036$ & $3.0531 \pm 0.0035$ \\
\hline$H_{0}$ & $66.90 \pm 0.51$ & $66.90 \pm 0.15$ & $66.90 \pm 0.11$ & $66.90 \pm 0.10$ \\
$\sigma_{8}$ & $0.8285 \pm 0.0040$ & $0.8285 \pm 0.0014$ & $0.8285 \pm 0.0011$ & $0.8285 \pm 0.0010$ \\
\hline
\end{tabular}

Table 5: Forecast 68\% CL constraints on primary and derived cosmological parameters when the running of the scalar spectral index is allowed to vary for LiteBIRD, LiteCORE-80, CORE-M5 and COrE + configurations. These forecasts assume $\mathrm{d} n_{\mathrm{s}} / \mathrm{d} \ln k=0$ as the fiducial value.

Fig. 7 shows instead the 2D forecast uncertainties when the spectral index is allowed to vary up to second order for a fiducial cosmology chosen based on the Planck 2015 TT,TE,EE + lowP best fit [5], i.e., $n_{\mathrm{s}}=0.9583, \mathrm{~d} n_{\mathrm{s}} / \mathrm{d} \ln k=0.015, \mathrm{~d}^{2} n_{\mathrm{s}} / \mathrm{d} \ln k^{2}=0.026$. For CORE-M5 (LiteBIRD) we obtain $\mathrm{d} n_{\mathrm{S}} / \mathrm{d} \ln k=0.0153 \pm 0.0025\left(\mathrm{~d} n_{\mathrm{s}} / \mathrm{d} \ln k=0.015 \pm 0.013\right)$ and $\mathrm{d}^{2} n_{\mathrm{s}} / \mathrm{d} \ln k^{2}=0.0261 \pm 0.0045$ $\left(\mathrm{d}^{2} n_{\mathrm{s}} / \mathrm{d} \ln k^{2}=0.026 \pm 0.014\right)$ at $68 \%$ CL (the uncertainties are essentially unchanged from the case with a fiducial case with no scale dependence of the spectral index). CORE-M5 has therefore the capability to probe at a statistically significant level the large running of the running 
which currently leads to an improved fit to data. ${ }^{9}$ The CORE-M5 capabilities to probe the scale dependence of the spectral index $n_{\mathrm{s}}$ down to slow-roll predictions will be further improved by future galaxy surveys [200].

\begin{tabular}{|c|c|c|c|c|}
\hline Parameter & LiteBIRD, TE & LiteCORE-80, TEP & CORE-M5, TEP & COrE,+ TEP \\
\hline$\Omega_{\mathrm{b}} h^{2}$ & $0.02215 \pm 0.00014$ & $0.022139 \pm 0.000061$ & $0.022141 \pm 0.000044$ & $0.022142 \pm 0.000039$ \\
$\Omega_{\mathrm{c}} h^{2}$ & $0.1206 \pm 0.0011$ & $0.12059 \pm 0.00040$ & $0.12058 \pm 0.00032$ & $0.12057 \pm 0.00031$ \\
$100 \theta_{\mathrm{MC}}$ & $1.03922 \pm 0.00030$ & $1.03922 \pm 0.00010$ & $1.039224 \pm 0.000077$ & $1.039222 \pm 0.000074$ \\
$\tau$ & $0.0583 \pm 0.0023$ & $0.0582 \pm 0.0021$ & $0.0582 \pm 0.0021$ & $0.0582 \pm 0.0020$ \\
$n_{\mathrm{s}}$ & $0.9625 \pm 0.0050$ & $0.9625 \pm 0.0026$ & $0.9625 \pm 0.0022$ & $0.9625 \pm 0.0021$ \\
$\mathrm{~d} n_{\mathrm{s}} / d \ln k$ & $0.000 \pm 0.014$ & $0.0001 \pm 0.0031$ & $0.0000 \pm 0.0024$ & $0.0000 \pm 0.0023$ \\
$\mathrm{~d}^{2} n_{\mathrm{s}} / d \ln k^{2}$ & $0.000 \pm 0.014$ & $-0.0003 \pm 0.0060$ & $0.0000 \pm 0.0046$ & $0.0000 \pm 0.0043$ \\
$\ln \left(10^{10} A_{\mathrm{s}}\right)$ & $3.0534 \pm 0.0055$ & $3.0532 \pm 0.0043$ & $3.0532 \pm 0.0040$ & $3.0533 \pm 0.0038$ \\
\hline$H_{0}$ & $66.90 \pm 0.50$ & $66.89 \pm 0.17$ & $66.90 \pm 0.13$ & $66.89 \pm 0.13$ \\
$\sigma_{8}$ & $0.8284 \pm 0.0055$ & $0.8285 \pm 0.0021$ & $0.8285 \pm 0.0016$ & $0.8285 \pm 0.0015$ \\
\hline
\end{tabular}

Table 6: Forecast 68\% CL constraints on primary and derived cosmological parameters when the scale dependence of the scalar spectral index is allowed to vary up to its second derivative for the LiteBIRD, LiteCORE-80, CORE-M5, and COrE + configurations. These forecasts assume $\mathrm{d} n_{\mathrm{s}} / \mathrm{d} \ln k=\mathrm{d}^{2} n_{\mathrm{s}} / \mathrm{d} \ln k^{2}=$ 0 as the fiducial values.

\subsection{Joint forecasts for $n_{\mathrm{s}}$ and $r$}

Only recently has the constraint on $r$ from the B-mode polarization become competitive with the constraint obtained using the temperature and E-mode polarization anisotropies alone. The BICEP 2/Keck Array/Planck (BKP) joint cross-correlation provided the same upper bound as that obtained with the Planck nominal mission temperature data in combination with the WMAP large angular scale polarization [3], i.e., $r<0.12$ at $95 \%$ CL [54]. The combination of Planck 2015 temperature and E-mode polarization data with the BKP cross-correlation tightened this constraint to $r<0.08$ at $95 \% \mathrm{CL}[4,5]$, thus strongly disfavoring what was believed to be the simplest inflationary model, i.e., a chaotic model with a quadratic potential $V(\phi) \propto \phi^{2}$ [5]. See [172] for the most recent BICEP 2/Keck Array/Planck joint cross-correlation including the Keck Array $95 \mathrm{GHz}$ and the WMAP 23, $33 \mathrm{GHz}$ bands, slightly improving the 95\% CL upper bound on $r$ to 0.07 .

With its mean noise level of $2 \mu K$ - arcmin and its angular resolution of 5' at $200 \mathrm{GHz}, \mathrm{CORE}$ M5 has the capability to probe several classes of large-field inflationary models, for which the excursion of the inflaton between the time at which the observable wavelength exited the Hubble radius and the end of inflation is of the order of the Planck mass. We first consider $r=10^{-3}$ as a fiducial value, which was discussed in Section 2 as an interesting target motivated by recent developments in supergravity [201]. We sample linearly on the six cosmological parameters and on the tensor-to-scalar ratio $r$ at the pivot scale $k_{*}=0.05 \mathrm{Mpc}^{-1}$, with the tensor tilt satisfying $n_{\mathrm{t}}=$

\footnotetext{
${ }^{9}$ Note that CORE-M5 forecast uncertainties on the running of the running also improve on the capabilities of future missions dedicated to the measurements of CMB spectral distortions as PIXIE, which can probe smaller wavelengths inaccessible using CMB anisotropies because of Silk damping. The forecast $68 \%$ uncertainties for $\mathrm{d} n_{\mathrm{s}} / \mathrm{d} \ln k$ and $\mathrm{d}^{2} n_{\mathrm{s}} / \mathrm{d} \ln k^{2}$ reported in Ref. [199] by combining Planck and spectral distortions by PIXIE are 0.0065 and 0.0045 , respectively, (i.e., slightly larger than those reported for CORE-M5).
} 
$-r / 8$ as predicted by canonical single field slow-roll models. The results for LiteBIRD, LiteCORE80, CORE-M5, and COrE + are presented in Table 7. Note that the LiteCORE-80, CORE-M5, and $\mathrm{COrE}+$ results include either internal $\mathrm{CMB}$ delensing for B-mode polarization and the CMB lensing as an additional observable in the mock likelihood. The noise sensitivity and the CMB $\mathrm{x}$ CMB delensing capability leads to $r=0.00100 \pm 0.00021$ at $68 \%$ CL for CORE-M5. The COREM5 uncertainty is nearly half of $3.6 \times 10^{-4}$, which can be reached by LiteBIRD and improves on that obtained by LiteCORE- 80 by $29 \%$. The more ambitious COrE + configuration improves on the CORE-M5 uncertainty by $10 \%$. As an approximate idea of the impact of foreground uncertainties, let us note that a Fisher matrix approach including foreground residuals and delensing [168] leads to an uncertainty on the tensor-to-scalar ratio as $\sigma(r) \approx 3.2 \times 10^{-4}$ for a fiducial case with $r=10^{-3}$ for CORE-M5.

CORE-M5 will target the Starobinsky $R^{2}$ model [202-204], which predicts $r \approx 12 / N_{*}^{2}$ (approximatively $r \sim 0.0042$ for $N_{*} \approx 53$ ). The Starobinsky model is currently the simplest among the inflationary models allowed by Planck data [3, 5, 205] and has been at the center stage of several theoretical developments in supergravity [78, 81, 82, 206], as explained in Section 2. By assuming $r=0.0042$ as the fiducial value, compatible with the Starobinsky $R^{2}$ model for the fiducial value of $n_{\mathrm{s}}$ considered in Section 3, we obtain $n_{\mathrm{s}}=0.9625 \pm 0.0030, r=0.00424 \pm 0.00057$, and $n_{\mathrm{s}}=0.9625 \pm 0.0015, r=0.00421 \pm 0.00028$ at $68 \% \mathrm{CL}$, for LiteBIRD and CORE-M5, respectively.

In the worst case scenario in which the energy scale of inflation is such that the primordial B-mode polarization is below the sensitivity of any experimental configuration studied here, we find $1.8 \times 10^{-4}$ and $4.3 \times 10^{-4}$ as the CORE-M5 and LiteBIRD 95\% CL upper bounds on $r$ taking into account noise sensitivity only, respectively. CORE-M5 could improve by more than a factor of 2 on the $95 \%$ CL upper bound on $r$ obtained with LiteBIRD.

\begin{tabular}{|c|c|c|c|c|}
\hline Parameter & LiteBIRD, TEB & LiteCORE-80, TEBP & CORE-M5, TEBP & COrE,+ TEBP \\
\hline$\Omega_{\mathrm{b}} h^{2}$ & $0.02215 \pm 0.00010$ & $0.022136 \pm 0.000057$ & $0.022141 \pm 0.000037$ & $0.022140 \pm 0.000034$ \\
$\Omega_{\mathrm{c}} h^{2}$ & $0.12056 \pm 0.00046$ & $0.12058 \pm 0.00033$ & $0.12058 \pm 0.00026$ & $0.12059 \pm 0.00025$ \\
$100 \theta_{\mathrm{MC}}$ & $1.03922 \pm 0.00028$ & $1.03923 \pm 0.000010$ & $1.039223 \pm 0.000077$ & $1.039223 \pm 0.000072$ \\
$\tau$ & $0.0582_{-0.0020}^{+0.0022}$ & $0.0582 \pm 0.0020$ & $0.0582 \pm 0.0020$ & $0.0582 \pm 0.0019$ \\
$n_{\mathrm{s}}$ & $0.9626 \pm 0.0030$ & $0.9625 \pm 0.0016$ & $0.9625 \pm 0.0015$ & $0.9625 \pm 0.0014$ \\
$\ln \left(10^{10} A_{\mathrm{s}}\right)$ & $3.0531 \pm 0.0041$ & $3.0531 \pm 0.0034$ & $3.0532 \pm 0.0034$ & $3.0531 \pm 0.0034$ \\
$r$ & $0.00104 \pm 0.00036$ & $0.00103 \pm 0.00027$ & $0.00100 \pm 0.00021$ & $0.00101 \pm 0.00019$ \\
\hline$H_{0}$ & $66.91 \pm 0.24$ & $66.90 \pm 0.14$ & $66.90 \pm 0.11$ & $66.90 \pm 0.10$ \\
$\sigma_{8}$ & $0.8284 \pm 0.0021$ & $0.8285 \pm 0.0014$ & $0.8285 \pm 0.0011$ & $0.8285 \pm 0.0010$ \\
\hline
\end{tabular}

Table 7: Forecast $68 \%$ CL constraints on primary and derived cosmological parameters when $r$ is allowed to vary for LiteBIRD, LiteCORE-80, CORE-M5, and COrE + configurations. These forecasts assume $r=$ $10^{-3}$ as the fiducial value.

The forecast CORE-M5 and LiteBIRD $68 \%$ and 95\% CL 2D marginalized regions in $\left(n_{\mathrm{s}}, r\right)$ for $r=0.0042, r=0.001$ and $r=0$ are displayed in Fig. 8. These forecasts are compared with the corresponding regions for the real Planck 2015 data combined with the BKP joint cross-correlation for B-mode polarization [5]. Theoretical predictions for a few inflationary models in agreement with current data are also shown: natural inflation (purple band), the hilltop quartic model 


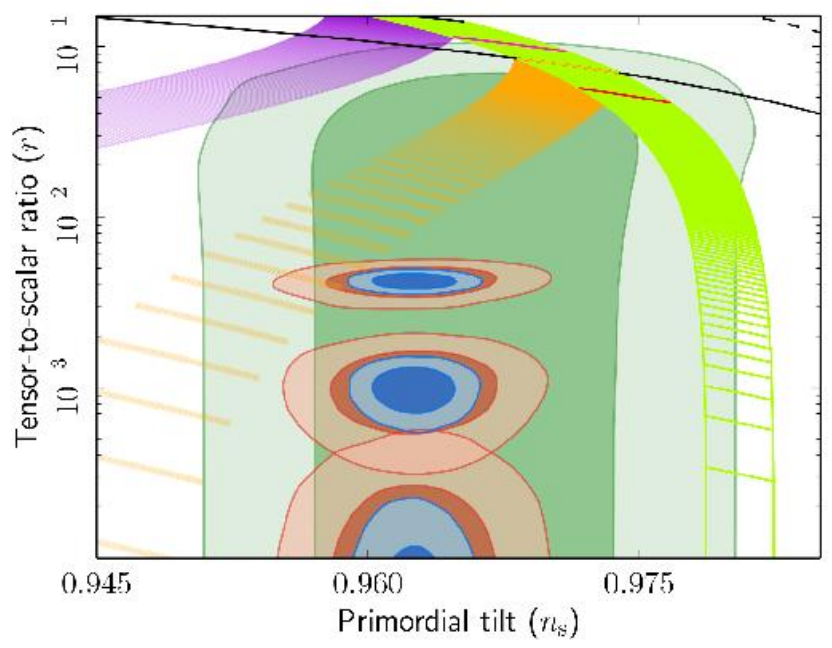

Figure 8: $68 \%$ and 95\% CL 2D marginalized forecast regions for $\left(n_{\mathrm{s}}, r\right)$ for CORE-M5 (blue) and LiteBIRD (red). Three reference cosmologies are considered: a value for the tensor-to-scalar ratio consistent with the $R^{2}$ model $(r \approx 0.0042), r=0.001$, and a third case in which the level of primordial gravitational waves is undetectably small (i.e., $r=0$ ). The green contours showing the $68 \%$ and $95 \%$ CL for Planck 2015 TT + lowP data combined with the BKP joint cross-correlation [5] are also displayed for comparison. We show the predictions for natural inflation [207, 208] (purple band), the hilltop quartic model [209] (orange discrete band), and power law chaotic [35] (light green discrete band) models, accounting for representative uncertainties in the post-inflationary era with $47<N_{*}<57$. These inflationary models consistent with the current data can be ruled out by CORE-M5. Note the logarithmic scale on the $y$-axis and that the pivot scale considered here is $k_{*}=0.05 \mathrm{Mpc}^{-1}$.

(orange discrete band), and power law chaotic models (light green discrete band), all displayed with standard representative uncertainties for the post-inflationary era. Fig. 8 shows how COREM5 can discriminate better than LiteBIRD among models which fit current observations at a similar statistical significance [5].

We now analyze the case in which either the scalar and tensor power spectra have a nonvanishing running of the spectral index. We restrict ourselves to the standard slow-roll case and we use consistency conditions for the tensor tilt and its running to the next-to-leading order, i.e., $n_{\mathrm{t}}=-r\left(2-r / 8-n_{\mathrm{s}}\right) / 8$ and $\mathrm{d} n_{\mathrm{t}} / \mathrm{d} \ln k=r\left(r / 8+n_{\mathrm{s}}-1\right) / 8$ at $k_{*}=0.05 \mathrm{Mpc}^{-1}$. When $r$ and $\mathrm{d} n_{\mathrm{s}} / \mathrm{d} \ln k$ are allowed to vary, the following constraints have been obtained with Planck $2015 \mathrm{TT}$ $+\operatorname{lowP}+\operatorname{BKP}[5]: n_{\mathrm{s}}=0.9661 \pm 0.0064, \mathrm{~d} n_{\mathrm{s}} / \mathrm{d} \ln k=-0.012_{-0.008}^{+0.009}$ at $68 \%$ CL and $r<0.09$ at $95 \%$ CL.

Table 8 reports the forecasts obtained by assuming $r=0.0042$ and $\mathrm{d} n_{\mathrm{s}} / \mathrm{d} \ln k=-0.0007$ as fiducial values, which are representative for the Starobinsky $R^{2}$ model [202-204] including the running of the spectral index. The CORE-M5 forecast uncertainties for the running are approximatively a factor of 3.5 smaller than the current uncertainties, whereas LiteBIRD will be able to provide only a $30 \%$ improvement. Allowing a non-vanishing running in the fit, the forecast errors on $n_{\mathrm{s}}$ increase by $23 \%$ for LiteBIRD, but just a $7 \%$ for LiteCORE-80, CORE-M 5 and $\mathrm{COrE}+$, with respect to the case of power law spectra. The forecast uncertainties reported in Tables 5 and 8 show that none of the configurations alone will allow precise measurements of running down to the level of $\mathrm{d} n_{\mathrm{s}} / \mathrm{d} \ln k \sim\left(n_{\mathrm{s}}-1\right)^{2} / 2$, but the CORE configurations will certainly be more powerful when combined with future large scale structure or $21 \mathrm{~cm}$ observations [197, 200]. 


\begin{tabular}{|c|c|c|c|c|}
\hline Parameter & LiteBIRD, TEB & LiteCORE-80, TEBP & CORE-M5, TEBP & COrE,+ TEBP \\
\hline$\Omega_{\mathrm{b}} h^{2}$ & $0.02214 \pm 0.00011$ & $0.022140 \pm 0.000060$ & $0.022140 \pm 0.000043$ & $0.022140 \pm 0.000038$ \\
$\Omega_{\mathrm{c}} h^{2}$ & $0.12057 \pm 0.00047$ & $0.12058 \pm 0.00033$ & $0.12058 \pm 0.00027$ & $0.12059 \pm 0.00026$ \\
$100 \theta_{\mathrm{MC}}$ & $1.03922 \pm 0.00028$ & $1.039223 \pm 0.000099$ & $1.039224 \pm 0.000077$ & $1.039222 \pm 0.000073$ \\
$\tau$ & $0.0583 \pm 0.0021$ & $0.0582 \pm 0.0020$ & $0.0582 \pm 0.0020$ & $0.0582 \pm 0.0019$ \\
$n_{\mathrm{s}}$ & $0.9625 \pm 0.0037$ & $0.9625 \pm 0.0017$ & $0.9625 \pm 0.0016$ & $0.9625 \pm 0.0015$ \\
$\mathrm{~d} n_{\mathrm{s}} / \mathrm{d} \ln k$ & $-0.0007 \pm 0.0059$ & $-0.0007 \pm 0.0030$ & $-0.0007 \pm 0.0024$ & $-0.0007 \pm 0.0023$ \\
$\ln \left(10^{10} A_{\mathrm{s}}\right)$ & $3.0532 \pm 0.0045$ & $3.0532 \pm 0.0037$ & $3.0532 \pm 0.0035$ & $3.0532 \pm 0.0035$ \\
$r$ & $0.0043 \pm 0.0006$ & $0.0042 \pm 0.0004$ & $0.00421 \pm 0.00028$ & $0.00421 \pm 0.00026$ \\
\hline$H_{0}$ & $66.91 \pm 0.25$ & $66.90 \pm 0.15$ & $66.89 \pm 0.11$ & $66.89 \pm 0.11$ \\
$\sigma_{8}$ & $0.8283 \pm 0.0025$ & $0.8283 \pm 0.0014$ & $0.8283 \pm 0.0011$ & $0.8283 \pm 0.0010$ \\
\hline
\end{tabular}

Table 8: Forecast $68 \%$ CL constraints on the primary and derived cosmological parameters when $r$ and the running of the spectral index are allowed to vary for the LiteBIRD, LiteCORE-80, and CORE-M5 configurations. These forecasts assume $r=0.0042$ and $\mathrm{d} n_{\mathrm{s}} / d \ln k=-0.0007$ as the fiducial values.
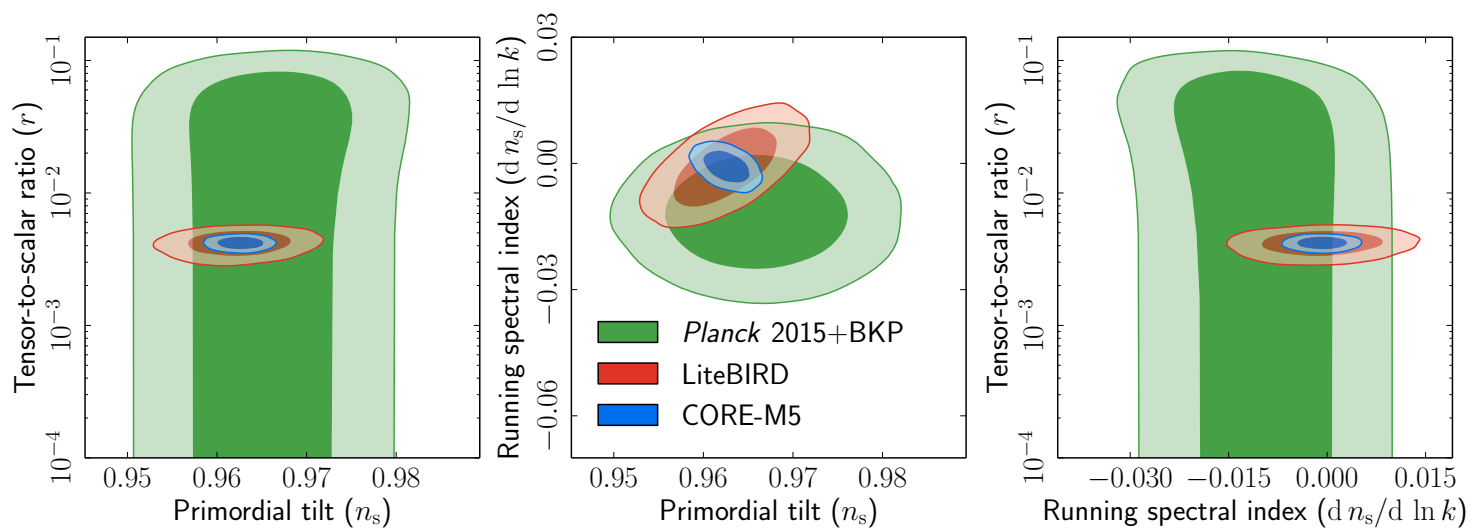

Figure 9: Forecast $68 \%$ and 95\% CL 2D marginalized regions for $\left(n_{\mathrm{s}}, r\right)$ (left panel), $\left(n_{\mathrm{s}}, \mathrm{d} n_{\mathrm{s}} / d \ln k\right)(\operatorname{middle}$ panel) and $\left(r, \mathrm{~d} n_{\mathrm{s}} / d \ln k\right)$ (right panel) for CORE-M5 (blue) and LiteBIRD (red) obtained by allowing the tensorto-scalar ratio and the running to vary. These forecasts assume $r=0.0042$ and $d n_{\mathrm{s}} / d \ln k=-0.0007$ as the fiducial values. The green contours show the $68 \%$ and $95 \%$ CL for Planck 2015 data combined with the BKP joint cross-correlation [5] are shown for comparison. Note that the Planck 2015 contours are based on real data whose best fit is different from the fiducial cosmology used in this paper.

We now study the dependence of the constraint on $\left(n_{\mathrm{s}}, r\right)$ with respect to uncertainties in the neutrino physics. See the companion paper on cosmological parameters [9] for an extensive study of how CORE-M5 could improve our current knowledge of neutrino physics. We consider as an example the case in which the number of neutrino species $N_{\text {eff }}$ is allowed to vary around the standard value 3.046. It is known that the damping tail of the power spectra of CMB anisotropies is very sensitive to the number of neutrino species and therefore partially degenerate with the scalar spectral index. The impact of the degeneracy of a varying $N_{\text {eff }}$ with $n_{\mathrm{s}}$ on establishing the statistical significance of the departure from scale invariance and on the combined constraints on $\left(n_{\mathrm{s}}, r\right)$ has been studied for the Planck data $[3,5]$. When $N_{\text {eff }}$ is allowed to vary simultaneously with the tensor amplitude, the following constraints are obtained using the Planck 2015 temperature and polarization data [5]: $n_{\mathrm{s}}=0.964 \pm 0.010, N_{\mathrm{eff}}=3.02_{-0.21}^{+0.20}$ at $68 \%$ CL. The constraint on $r$ is 

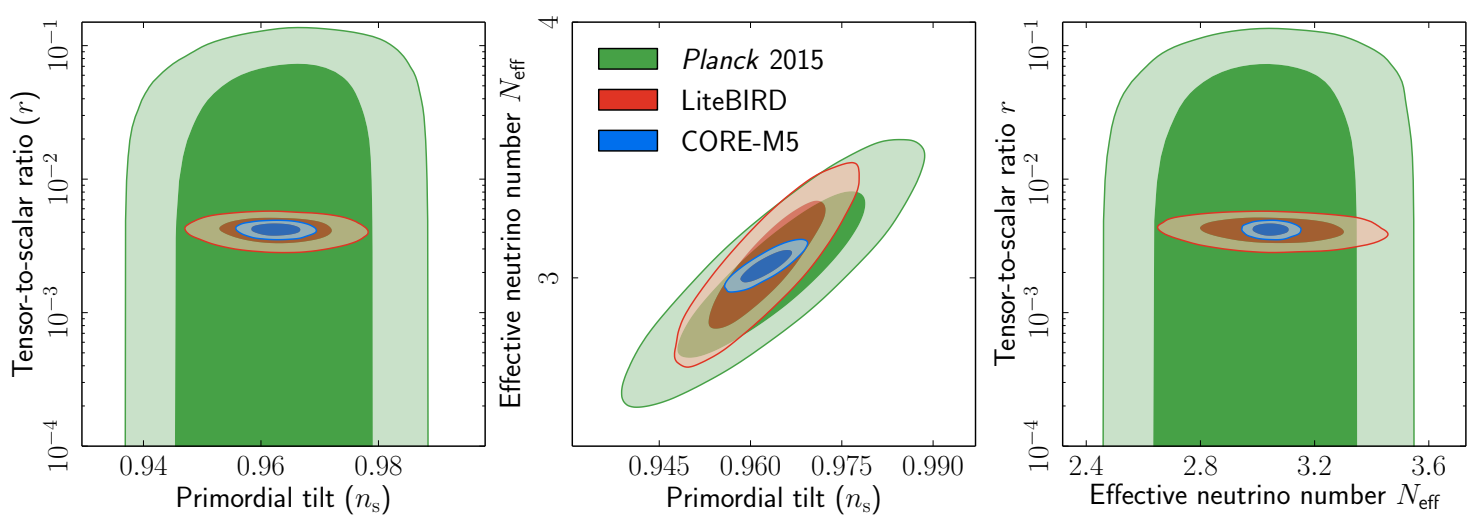

Figure 10: Forecast $68 \%$ and $95 \%$ CL 2D marginalized regions for the $\left(n_{\mathrm{s}}, r\right)$ (left panel), $\left(n_{\mathrm{s}}, N_{\text {eff }}\right)$ (middle panel) and $\left(N_{\text {eff }}, r\right)$ (right panel) for CORE-M5 (blue) and LiteBIRD (red) obtained by allowing the tensor-to-scalar ratio and the running to vary. These forecasts assume $r=0.0042$ and $N_{\text {eff }}=3.046$ as fiducial values. The $68 \%$ and $95 \%$ CL marginalized contours for Planck 2015 TT,TE,EE + lowP (green) are shown for comparison [5]. Note that the Planck 2015 contours are based on real data whose best fit is different from the fiducial cosmology used in this paper.

unchanged by allowing $N_{\text {eff }}$ to vary, i.e., $r<0.11$ at $95 \%$ CL.

Table 9 reports the forecasts for primary and derived parameters when $r$ and $N_{\text {eff }}$ are simultaneously varied in addition to the parameters of the standard $\Lambda$ CDM model, assuming a fiducial model with $r=0.0042$ and $N_{\text {eff }}=3.046$. LiteBIRD improves on the Planck uncertainties by approximatively $40 \%$ in $n_{\mathrm{s}}$ and $20 \%$ in $N_{\text {eff }}$. By contrast, CORE-M5 has the capability to reduce by approximatively a factor of 4 the Planck 2015 uncertainties on $n_{\mathrm{s}}$ and $N_{\text {eff }}$, respectively. LiteCORE-80 forecasts are slightly worst than CORE-M5, whereas COrE + can further improve the CORE-M5 forecasts on $n_{\mathrm{s}}$ and $N_{\text {eff }}$ by $3.7 \%$ and $7.5 \%$, respectively. See Fig. 9 for the 2D joint 68 and $95 \%$ CL marginalized regions for $\left(n_{\mathrm{s}}, r\right),\left(n_{\mathrm{s}}, N_{\text {eff }}\right)$ and $\left(N_{\text {eff }}, r\right)$ expected from LiteBIRD and CORE-M5, compared with the current Planck 2015 results.

\begin{tabular}{|c|c|c|c|c|}
\hline Parameter & LiteBIRD, TEB & LiteCORE-80, TEBP & CORE-M5, TEBP & COrE,+ TEBP \\
\hline$\Omega_{\mathrm{b}} h^{2}$ & $0.02215 \pm 0.00019$ & $0.022142 \pm 0.000085$ & $0.022141 \pm 0.000057$ & $0.022141 \pm 0.000049$ \\
$\Omega_{\mathrm{c}} h^{2}$ & $0.1207 \pm 0.0022$ & $0.12062 \pm 0.00093$ & $0.12060 \pm 0.00061$ & $0.12060 \pm 0.00057$ \\
$100 \theta_{\mathrm{MC}}$ & $1.03921 \pm 0.00041$ & $1.039223 \pm 0.000099$ & $1.039221 \pm 0.000091$ & $1.039222 \pm 0.000085$ \\
$\tau$ & $0.0582 \pm 0.0020$ & $0.0582 \pm 0.0020$ & $0.0582 \pm 0.0019$ & $0.0581 \pm 0.0018$ \\
$n_{\mathrm{s}}$ & $0.9626 \pm 0.0060$ & $0.9626 \pm 0.0028$ & $0.9625 \pm 0.0027$ & $0.9625 \pm 0.0026$ \\
$\ln \left(10^{10} A_{\mathrm{s}}\right)$ & $3.053 \pm 0.071$ & $3.0533 \pm 0.0038$ & $3.0532 \pm 0.0035$ & $3.0531 \pm 0.0035$ \\
$r$ & $0.00424 \pm 0.00057$ & $0.00421 \pm 0.00038$ & $0.00421 \pm 0.00028$ & $0.00421 \pm 0.00026$ \\
$N_{\text {eff }}$ & $3.05 \pm 0.16$ & $3.048 \pm 0.045$ & $3.046 \pm 0.040$ & $3.046 \pm 0.037$ \\
\hline$H_{0}$ & $66.9 \pm 1.1$ & $66.90 \pm 0.34$ & $66.90 \pm 0.31$ & $66.89 \pm 0.28$ \\
$\sigma_{8}$ & $0.8284 \pm 0.0058$ & $0.8284 \pm 0.0023$ & $0.8284 \pm 0.0020$ & $0.8283 \pm 0.0019$ \\
\hline
\end{tabular}

Table 9: Forecast 68\% CL constraints on primary and derived cosmological parameters when $r$ and $N_{\text {eff }}$ are allowed to vary for the LiteBIRD, LiteCORE-80, CORE-M5, and COrE + configurations. These forecasts assume $r=0.0042$ and $N_{\text {eff }}=3.046$ as fiducial values. 

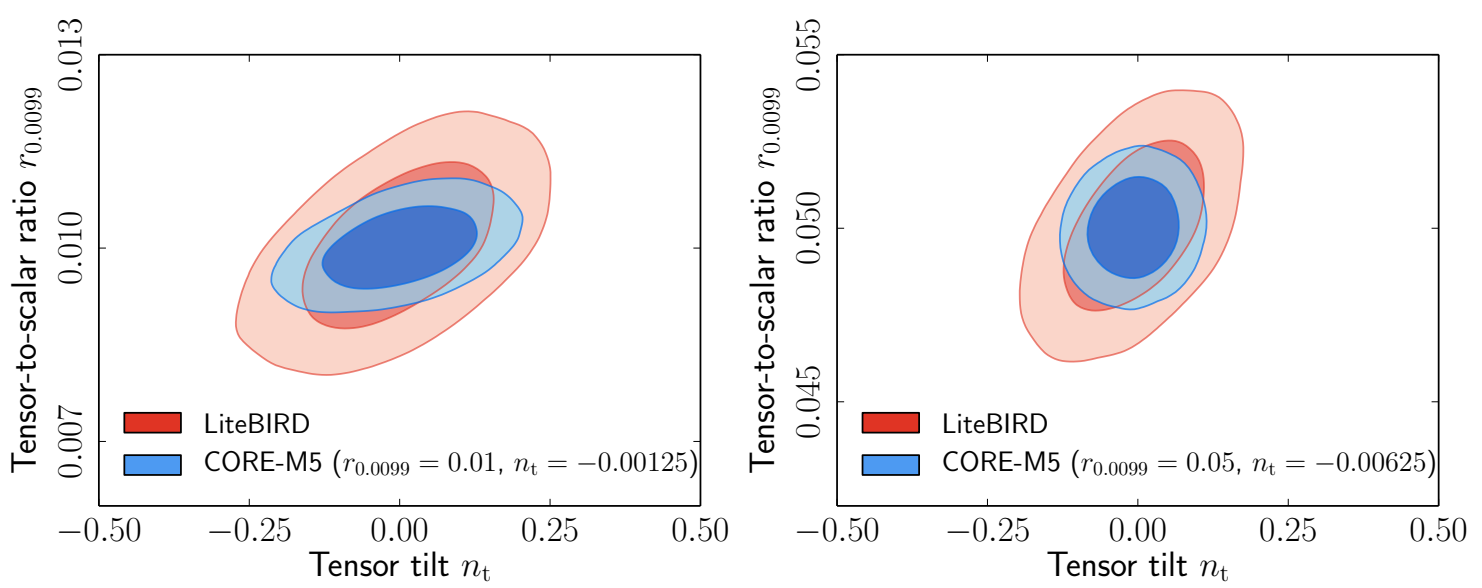

Figure 11: Forecasts for the $\left(n_{\mathrm{t}}, r\right)$ marginalized regions at the $68 \%$ and $95 \%$ CL for CORE-M5 (blue) and LiteBIRD (red) considering $r=0.01, n_{\mathrm{t}}=-r / 8=-0.00125$ (left panel) and $r=0.05, n_{\mathrm{t}}=-r / 8=-0.00625$ (right panel). The tensor pivot scale is $k_{*, \mathrm{t}}=0.0099 \mathrm{Mpc}^{-1}$.

\subsection{Beyond the consistency condition for the tensor tilt}

The consistency condition for the tensor tilt predicted by canonical single field slow-roll inflation is a key theoretical prior commonly assumed given the current sensitivity of CMB anisotropies measurements to the tensor contribution. Deviations from $n_{\mathrm{t}} \approx-r / 8$ are expected for well motivated extensions of the simplest scalar field inflationary models, which include generalized Lagrangians [210, 211], multifield inflation [212-214], and gauge inflation [215]. More radical departures from a nearly scale-invariant Gaussian spectrum of gravitational waves are expected in presence of non-vanishing anisotropic stresses from additional components during inflation [216] (see [217] for a recent review) or in alternative models to inflation [218-220].

In case of a statistically significant detection of primordial B-mode polarization, the next observational target would be the measurement of the tensor tilt $n_{\mathrm{t}}$. As a benchmark for our assessment of the expected uncertainties on the tensor tilt, we consider $\left(r=0.05, n_{\mathrm{t}}=-r / 8=\right.$ $-0.00625)$ and $\left(r=0.01, n_{\mathrm{t}}=-r / 8=-0.00125\right)$ at $k_{*, \mathrm{t}}=0.0099 \mathrm{Mpc}^{-1} 10$ as fiducial values compatible with current constraints, and consistent with those considered in the S4 science book [68]. The two dimensional marginalized regions expected from CORE-M5 and LiteBIRD are shown in Fig. 11. As a forecast $68 \%$ CL uncertainty for the tensor tilt we obtain $\sigma\left(n_{\mathrm{t}}\right)=0.05(0.08)$ for $r=0.05$ and $\sigma\left(n_{\mathrm{t}}\right)=0.08$ (0.1) for $r=0.01$ with CORE-M5 (LiteBIRD) specifications. The CORE-M5 uncertainties on $r$ remain basically unchanged when we allow for free $n_{\mathrm{t}}(\sigma(r)=0.0009$ and $\sigma(r)=0.0004$ for $r=0.05$ and $r=0.01$ as fiducial values, respectively). Although the verification of the consistency relation for the tensor tilt was expected out of reach for values consistent with the current upper bounds on $r$ [168, 221], the CORE capability to probe either the recombination and the reionization peak is a key aspect for measuring the slope of the tensor power spectrum.

\footnotetext{
${ }^{10}$ In this Section we consider two different pivot scales for scalar and tensor power spectra in Eqs. (4.1) and (4.2). We retain the standard pivot scale at $0.05 \mathrm{Mpc}^{-1}$ for the scalar perturbations.
} 


\subsection{Constraints on the curvature}

Since the measurement of the first acoustic peak of the CMB temperature power spectrum [37], the consistency of cosmological observations with a flat Universe has been an important test in favor of inflation. Planck data set tight constraints on the spatial curvature at the percent level. The Planck 2015 result $\Omega_{\mathrm{k}}=-0.040_{-0.016}^{+0.024}$ at $68 \%$ CL obtained by temperature and polarization data is further tightened by the inclusion of Planck lensing, i.e., $\Omega_{\mathrm{k}}=-0.004_{-0.007}^{+0.008}[4,45]$, or by the inclusion of BAO [4]. We now discuss the capabilities of the different experimental configurations for a future $\mathrm{CMB}$ space mission in constraining the spatial curvature.

As from Table 10, the LiteBIRD forecast uncertainty for $\Omega_{\mathrm{k}}$ based on TE does not improve on the current Planck constraints including lensing. The CORE-M5 forecast uncertainty based on TE is approximatively an order of magnitude better than the corresponding Planck constraint and six times better than what is expected from LiteBIRD. By including the lensing information, the CORE-M5 forecast uncertainty on $\Omega_{\mathrm{k}}$ further improves by approximatively $18 \%$. COrE + can further reduce the CORE-M5 uncertainty on $\Omega_{\mathrm{k}}$ by $5 \%$.

It is interesting to note that CORE-M5 will provide an accurate measurement of the $E$-mode polarization spectrum in the region of the acoustic peaks sufficient to remove almost completely the CMB degeneracy of $\Omega_{\mathrm{k}}$ with the other cosmological parameters. The amount of spatial curvature allowed by Planck 2015 temperature and polarization data and improving on the $\Lambda$ CDM fit with a $\Delta \chi^{2} \sim-5[4]$ will be therefore probed by CORE-M5 without the use of the lensing information. These improvements in the uncertainties of spatial curvature might open new avenues in the understanding of the global dynamics of inflation [222] or could transform our understanding of inflation showing that our Universe is inside a large bubble embedded in the background vacuum space.

\begin{tabular}{|c|c|c|c|c|}
\hline Parameter & LiteBIRD, TE & CORE-M5, TE & CORE-M5, TEP & COrE,+ TEP \\
\hline$\Omega_{\mathrm{b}} h^{2}$ & $0.02215 \pm 0.00014$ & $0.022140 \pm 0.000041$ & $0.022141 \pm 0.000040$ & $0.022141 \pm 0.000034$ \\
$\Omega_{\mathrm{c}} h^{2}$ & $0.1206 \pm 0.0010$ & $0.12059 \pm 0.00068$ & $0.12057 \pm 0.00067$ & $0.12057 \pm 0.00064$ \\
$100 \theta_{\mathrm{MC}}$ & $1.03922 \pm 0.00030$ & $1.039219 \pm 0.000086$ & $1.039219 \pm 0.000086$ & $1.039220 \pm 0.000081$ \\
$\tau$ & $0.0583 \pm 0.0021$ & $0.0582 \pm 0.0020$ & $0.0583 \pm 0.0020$ & $0.0582 \pm 0.0019$ \\
$n_{\mathrm{s}}$ & $0.9625 \pm 0.0034$ & $0.9626 \pm 0.0018$ & $0.9626 \pm 0.0018$ & $0.9626 \pm 0.0018$ \\
$\ln \left(1^{10} A_{\mathrm{s}}\right)$ & $3.0533 \pm 0.0046$ & $3.0532 \pm 0.0043$ & $3.0533 \pm 0.0043$ & $3.0532 \pm 0.0042$ \\
$\Omega_{\mathrm{k}}$ & $-0.001 \pm 0.012$ & $0.0001 \pm 0.0021$ & $0.0000 \pm 0.0019$ & $0.0000 \pm 0.0018$ \\
\hline$H_{0}$ & $66.8_{-6.1}^{+4.8}$ & $66.98 \pm 0.81$ & $66.90 \pm 0.65$ & $66.90 \pm 0.63$ \\
$\sigma_{8}$ & $0.827 \pm 0.010$ & $0.8286 \pm 0.0040$ & $0.8285 \pm 0.0040$ & $0.8285 \pm 0.0039$ \\
\hline
\end{tabular}

Table 10: Forecast $68 \%$ CL constraint on primary and derived cosmological parameters when the spatial curvature $\Omega_{\mathrm{k}}$ is allowed to vary for LiteBIRD and CORE-M5 without and with the lensing information, and $\mathrm{COrE}+$. These forecasts assume $\Omega_{\mathrm{k}}=0$ as fiducial value.

\subsection{Summary}

The forecasts presented in this Section show how the experimental configuration of the CORE mission proposed to ESA allows for a significant improvement on the uncertainties of key inflationary parameters with respect to the current measurements largely based on Planck. COREM5 performs better than the LiteCORE-80 and LiteCORE-120 configurations (the latter with a 

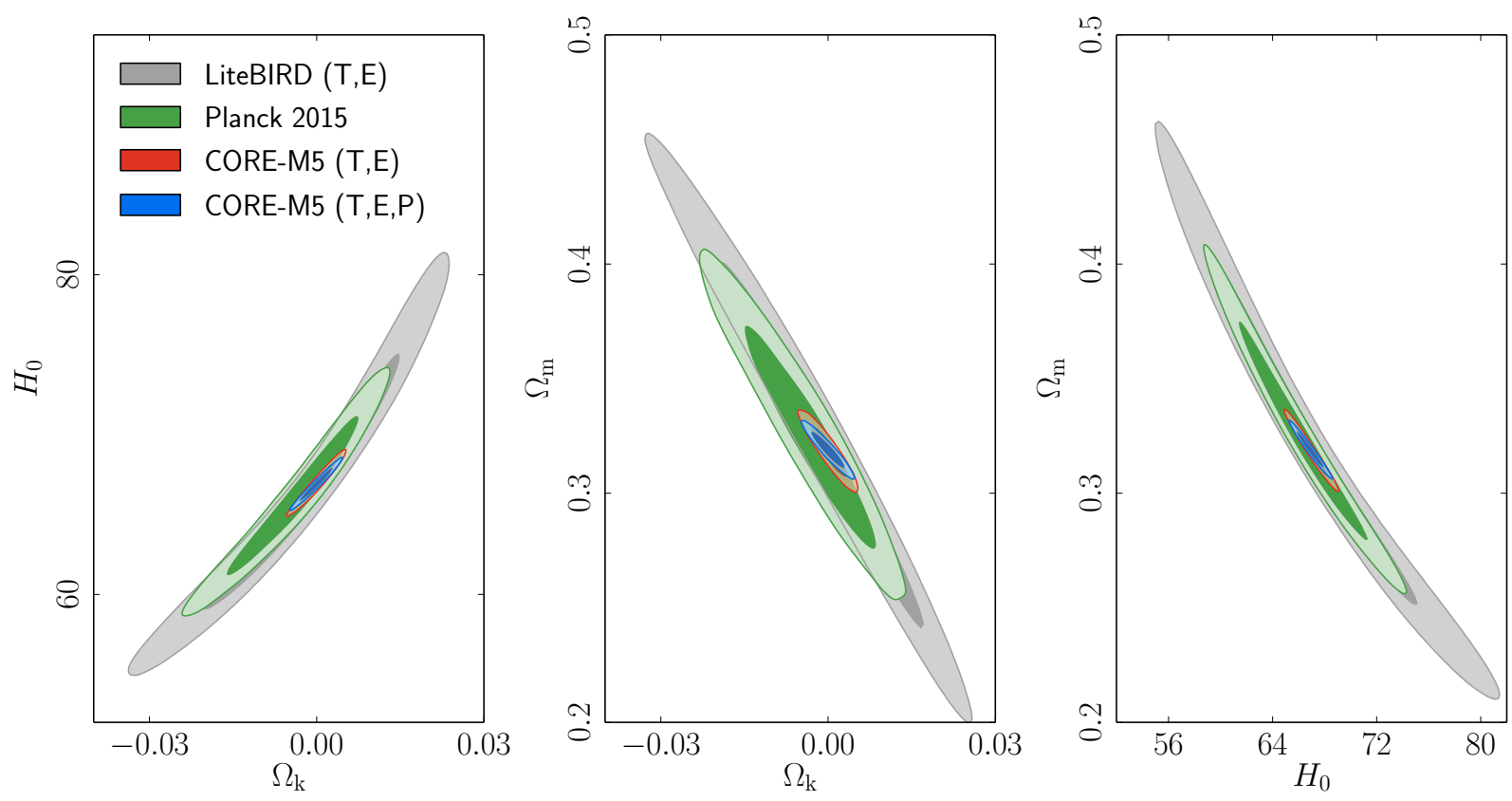

Figure 12: Forecast $68 \%$ and $95 \%$ CL marginalized regions for $\left(\Omega_{\mathrm{k}}, H_{0}\right)$ (left panel), $\left(\Omega_{\mathrm{k}}, \Omega_{\mathrm{m}}\right)$ (middle panel) and $\left(H_{0}, \Omega_{\mathrm{m}}\right)$ (right panel) for LiteBIRD (grey) and CORE-M5 (blue) obtained by allowing $\Omega_{\mathrm{k}}$ to vary. These forecasts assume $\Omega_{\mathrm{k}}=0$ as fiducial value. The $68 \%$ and $95 \%$ CL marginalized contours for Planck 2015 TT,TE,EE + lowP + lensing (green) are shown for comparison [4]. Note that the Planck 2015 contours are based on real data whose best fit is different from the fiducial cosmology used.

slightly larger telescope but higher noise due to the smaller number of detectors than CORE-M5). Thanks to a $1.5 \mathrm{~m}$ telescope, $\mathrm{COrE}+$ leads to an improvement with respect to CORE-M5, which is however modest compared to the higher cost connected to such larger mirror.

Our forecasts show that the LiteBIRD noise sensitivity (based on the LiteBIRD-ext configuration [168]) guarantees a measurement of the optical depth at the same level of the other configurations considered. Due to the smaller aperture, LiteBIRD provides an uncertainty on the spectral index $n_{\mathrm{s}}$ at least twice that of CORE-M5. Moreover, LiteBIRD will not provide a significant improvement with respect to the most recent Planck results [178] in the measurement of $\Omega_{\mathrm{b}} h^{2}, \theta_{\mathrm{MC}}$ (and therefore $H_{0}$ ), the scale dependence of the spectral index, and $\Omega_{\mathrm{k}}$. Concerning the search for primordial B-mode polarization, the LiteBIRD forecast uncertainty in $r$ is approximatively twice as large as from CORE-M5. The lower noise sensitivity and higher angular resolution of CORE-M5 becomes even more important in the comparison with LiteBIRD when theoretical assumptions are relaxed as the case of varying the effective number of neutrinos $N_{\text {eff }}$ shows.

\section{Constraints on slow-roll inflationary models}

At present, the full set of CMB temperature and polarization anisotropy measurements [5, 55, 223,224 ] can be accounted for in a minimal setup, where inflation is driven by a single scalar field $\phi$ with a canonical kinetic term, minimally coupled to gravity, and evolving in a flat potential 
$V(\phi)$. In this slow-roll regime, the dynamics are often parameterized in terms of the HFFs

$$
\epsilon_{n+1}=\frac{\mathrm{d} \ln \epsilon_{n}}{\mathrm{~d} N}
$$

where $\epsilon_{0}=H_{\text {in }} / H$ and $N=\ln a$ is the number of $e$-folds. The values of the $\epsilon_{n}$ parameters characterize the predictions of each model. At second order in slow roll, one has $n_{\mathrm{s}}=1-2 \epsilon_{1}-$ $\epsilon_{2}-2 \epsilon_{1}^{2}-(2 C+3) \epsilon_{1} \epsilon_{2}-C \epsilon_{2} \epsilon_{3}, r=16 \epsilon_{1}+16 C \epsilon_{1} \epsilon_{2}$ and $\mathrm{d} n_{\mathrm{s}} / \mathrm{d} \ln k=-2 \epsilon_{1} \epsilon_{2}-\epsilon_{2} \epsilon_{3}$, where the parameter $C \approx-0.73$ is a numerical constant. In Sec. 5.1.1, we derive forecasts on the parameters $\epsilon_{i}$ in various experimental configurations.

Since particle physics beyond the electroweak scale remains elusive, and given that inflation can proceed at energy scales as large as $10^{16} \mathrm{GeV}$, hundreds of inflationary scenarios have been proposed. A systematic Bayesian analysis for a selection of inflationary models listed in Ref. [102] has revealed that one third of them can now be considered as ruled out [102, 205, 225], while the vast majority of the preferred scenarios are of the plateau type, i.e., they are such that the potential $V(\phi)$ is a monotonic function that asymptotes to a constant value when $\phi$ goes to infinity. This still leaves us with about 50 "favored" potentials of those listed in Ref. [102], corresponding to various extensions of the Standard Model of particle physics between which it is therefore still impossible to discriminate. In this Section, we quantify the ability of the proposed mission to improve this picture and to provide further insight into the physics of the very early Universe.

For reference, we will consider two fiducial models of inflation, both of the plateau type and belonging to the favored models according to the recent Planck measurements. The first is the Starobinsky model (SI), and corresponds to a case in which $r$ is sufficiently large so that $B$ modes should be detected with CORE. The second fiducial model is Mutated Hilltop Inflation (MHI), where the mass scale $\mu$ appearing in this potential has been taken to $\mu=0.01 M_{\mathrm{Pl}}$ (further details about these models can be found in Ref. [102]), and corresponds to a case in which $r$ is too small for $B$-modes to be detected. In both cases, the reheating temperature has been fixed to $T_{\text {reh }}=10^{8} \mathrm{GeV}$ with an average equation of state $\bar{w}_{\text {reh }}=0$, while the post-inflationary evolution is assumed to be described by a flat $\Lambda$ CDM model with $\Omega_{\mathrm{b}} h^{2}=0.0223, \Omega_{\mathrm{c}} h^{2}=0.120$, $\Omega_{\nu} h^{2}=6.45 \times 10^{-4}, \tau=0.0931$ and $h=0.674$. In this Section we have not folded in $C_{\ell}^{\mathrm{PP}}$. We have cross-checked our results with two independent numerical pipelines. The first combines the Einstein-Boltzmann code CLASS [226] and the sampling code MontePython [227], and the second relies on CAMB [175] combined with the sampler CosmoMC [179].

\subsection{Constraints on slow-roll parameters}

\subsubsection{Impact of apparatus size and sensitivity}

The $1 \mathrm{D}$ and $2 \mathrm{D}$ marginalized posterior distributions on the $\epsilon_{n}$ parameters after $\mathrm{CMBxCMB}$ delensing are displayed in Figs. 13 and 14. At leading order in slow roll, $\epsilon_{1}$ is given by $r / 16$, and since the order of magnitude of $r$ is unknown, a uniform $\log$ prior with $\log \epsilon_{1} \in[-7,-0.7]$ is used, while flat priors $\epsilon_{2}, \epsilon_{3} \in[-0.2,0.2]$ are employed. The corresponding $1 \sigma$ bounds or $2 \sigma$ upper limits are given in Table 11. In order to determine the optimal balance between the mirror size, the number of detectors, mission duration, and the total mission cost, an important task is to analyze how the forecasts for inflation are modified, first when varying the angular resolution 

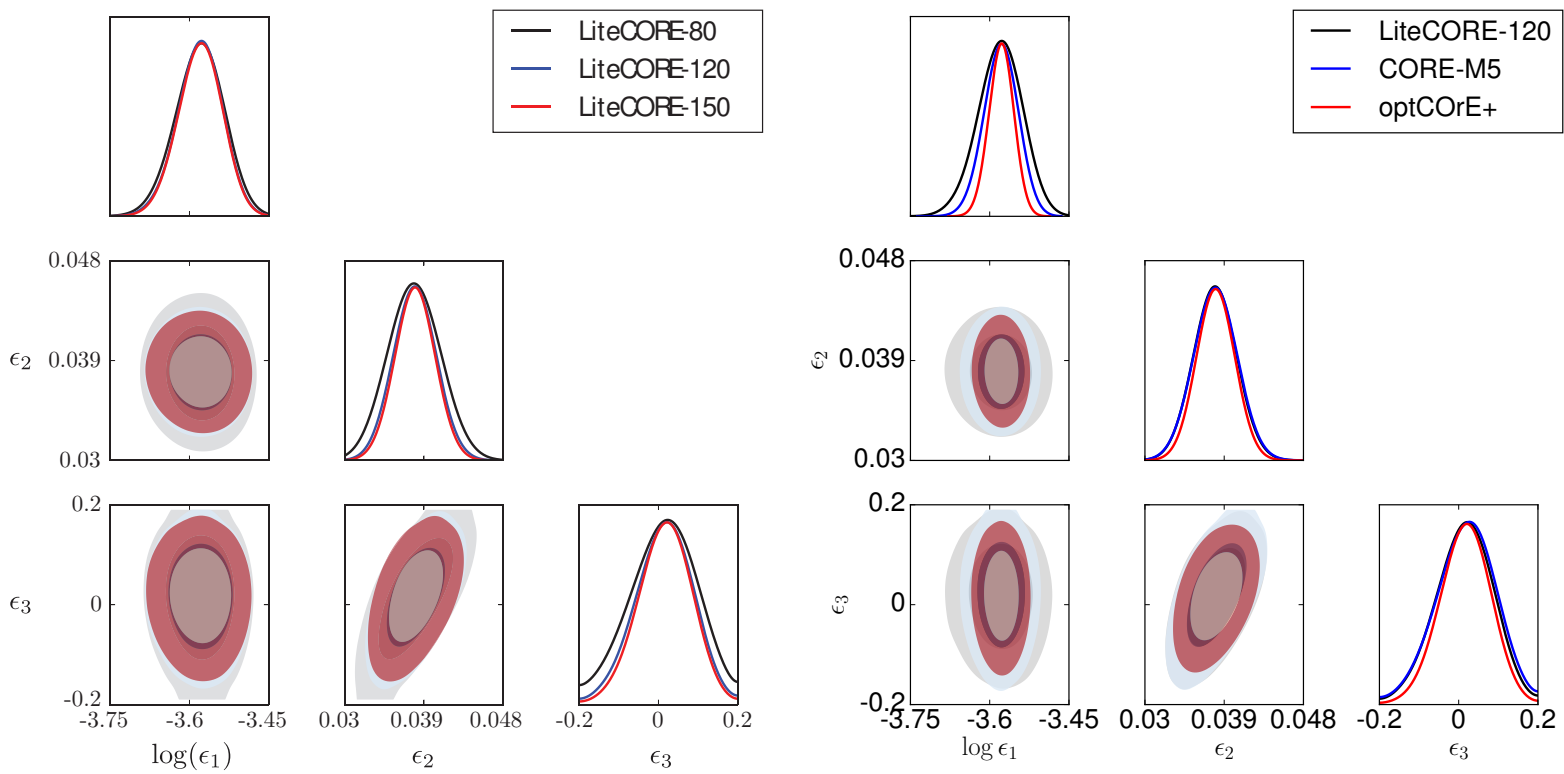

Figure 13: Compared forecasts on the Hubble flow parameters (1D and 2D marginalized posterior distributions after CMBxCMB delensing) when the fiducial model is SI.
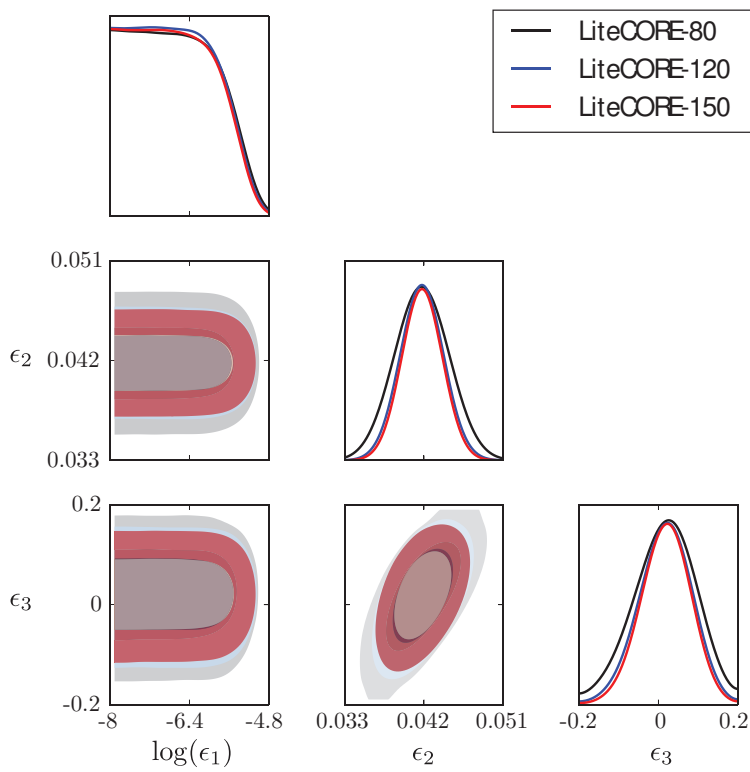

Figure 14: Compared forecasts on the Hubble flow parameters (1D and 2D marginalized posterior distributions after $\mathrm{CMBxCMB}$ delensing) when the fiducial model is MHI.

at fixed sensitivity, and second when improving or degrading the sensitivity, always by using the experimental configurations presented in Section 3.

One obtains very similar results for $\epsilon_{1}$ with LiteCORE-80, LiteCORE-120 and LiteCORE- 
SI fiducial model

\begin{tabular}{|c|c|c|c|c|c|}
\hline Parameter & LiteCORE-80 & LiteCORE-120 & LiteCORE-150 & CORE-M5 & optCOrE+ \\
\hline $\log \epsilon_{1}(-3.58)$ & $-3.581_{-0.040}^{+0.045}$ & $-3.580_{-0.037}^{+0.042}$ & $-3.580_{-0.036}^{+0.040}$ & $-3.579_{-0.029}^{+0.029}$ & $-3.578_{-0.021}^{+0.022}$ \\
\hline$\epsilon_{2}(0.0381)$ & $0.0379_{-0 .}^{+0 .(}$ & $0.0380_{-0}^{+0}$ & $0.0380_{-0.0021}^{+0.0021}$ & $0.0380_{-0.0022}^{+0.0022}$ & $0.0380_{-0.0020}^{+0.0019}$ \\
\hline$\epsilon_{3}(0.0193)$ & $0.013_{-0 .}^{+0 .}$ & $0.014_{-0}^{+0}$ & $0.016_{-0.062}^{+0.069}$ & $0.018_{-0.066}^{+0.078}$ & $0.016_{-0.059}^{+0.063}$ \\
\hline $\log r(-2.388)$ & $-2.387_{-0.040}^{+0.045}$ & $-2.385_{-0.037}^{+0.042}$ & $-2.386_{-0.036}^{+0.040}$ & $-2.387_{-0.029}^{+0.029}$ & $-2.386_{-0.021}^{+0.022}$ \\
\hline$n_{\mathrm{s}}(0.9619)$ & $0.9621_{-0.0021}^{+0.0021}$ & $0.9620_{-0.0020}^{+0.0020}$ & $0.9620_{-0.0020}^{+0.0019}$ & $0.9619_{-0.0022}^{+0.0022}$ & $0.9619_{-0.0019}^{+0.0019}$ \\
\hline$\imath_{\mathrm{s}} / \mathrm{d} \ln k(-0.0008)$ & $-0.0008_{-0.0030}^{+0.0031}$ & $-0.0008_{-0.0026}^{+0.0026}$ & $-0.0008_{-0.0024}^{+0.0024}$ & $-0.0008_{-0.0028}^{+0.0028}$ & $-0.0008_{-0.0023}^{+0.0023}$ \\
\hline \multicolumn{6}{|c|}{ MHI fiducial model } \\
\hline Parameter & LiteCORE-80 & LiteCORE-120 & LiteCORE-150 & CORE-M5 & optCOrE+ \\
\hline $\log \epsilon_{1}(-7.66)$ & $<-5.40$ & $<-5.44$ & $<-5.46$ & $<-5.50$ & $<-5.79$ \\
\hline$\epsilon_{2}(0.0419)$ & $0.0418_{-0.0029}^{+0.0029}$ & $0.0418_{-0.0023}^{+0.0023}$ & $0.0418_{-0.0021}^{+0.0021}$ & $0.0418_{-0.0023}^{+0.0022}$ & $0.0418_{-0.0020}^{+0.0020}$ \\
\hline$\epsilon_{3}(0.0209)$ & $0.016_{-0.070}^{+0.083}$ & $0.017_{-0.060}^{+0.069}$ & $0.019_{-0.057}^{+0.063}$ & $0.020_{-0.062}^{+0.071}$ & $0.018_{-0.054}^{+0.059}$ \\
\hline $\log r(-6.469)$ & $<-4.19$ & $<-4.24$ & -4.25 & $<-4.29$ & $<-4.58$ \\
\hline$n_{\mathrm{s}}(0.9587)$ & $0.9588_{-0.0021}^{+0.0021}$ & $0.9588_{-0.0020}^{+0.0020}$ & $0.9588_{-0.0020}^{+0.0019}$ & $0.9588_{-0.0022}^{+0.0022}$ & $0.9588_{-0.0019}^{+0.0019}$ \\
\hline $\mathrm{d} n_{\mathrm{s}} / \mathrm{d} \ln k(-0.0009)$ & $-0.0009_{-0.0031}^{+0.0031}$ & $-0.0009_{-0.0026}^{+0.0026}$ & $-0.0009_{-0.0024}^{+0.0024}$ & $-0.0009_{-0.0028}^{+0.0028}$ & $-0.0009_{-0.0023}^{+0.0023}$ \\
\hline
\end{tabular}

Table 11: Hubble flow parameters $1 \sigma$ bounds or $2 \sigma$ upper limit after CMBxCMB delensing. The corresponding constraints on the power spectra parameters $\log r, n_{\mathrm{s}}$ and $\mathrm{d} n_{\mathrm{s}} / \mathrm{d} \ln k$ are also given and have been obtained by importance sampling. The numbers in parenthesis in the first column are the fiducial values used for the corresponding parameters.

150, because these experimental configurations have the same sensitivity but different angular resolutions. For $\epsilon_{2}$ and $\epsilon_{3}$, some improvement occurs when going from LiteCORE-80 to LiteCORE120 , mainly because of the degraded angular resolution, which reduces the lever arm to probe the spectral tilt and the running of the scalar power spectrum. Otherwise, very similar results are obtained for LiteCORE-120 and LiteCORE-150. Hence there is no clear gain in increasing the mirror size at fixed sensitivity for diameters beyond $120 \mathrm{~cm}$.

On the other hand, the constraints on $\epsilon_{1}$ are improved when going from LiteCORE to COREM5 (by about $30 \%$ for the SI fiducial), and from CORE-M5 to optCOrE + (by about 25\% for SI). This illustrates the importance of the sensitivity of the experiment for recovering the first Hubble flow parameter. Nevertheless there is no significant improvement in the recovery of the second and third Hubble flow parameters.

We conclude that the sensitivity plays the dominant role, and thus the number and quality of detectors at the focal plane. The number of detectors typically scales with the size of the focal plane, and thus with the size of the experiment, which itself determines the angular resolution. Nevertheless, one finds that the angular resolution alone plays a minor role, as long as it suffices to probe the lensing spectrum and efficiently delens the B-mode. These results give strong support to the CORE-M5 specifications, which optimize the sensitivity through the number of detectors and the mission duration, for a slightly reduced mirror size compared to the previous $\mathrm{COrE}+$ specifications.

\subsubsection{Removing low multipoles and delensing}

The forecasts derived in Section 5.1.1 rely on the assumption that low multipoles can be correctly measured and that delensing can be performed successfully. However, these two tasks can be challenging in practice, and this is why in this Subsection we assess the degradation of our results 


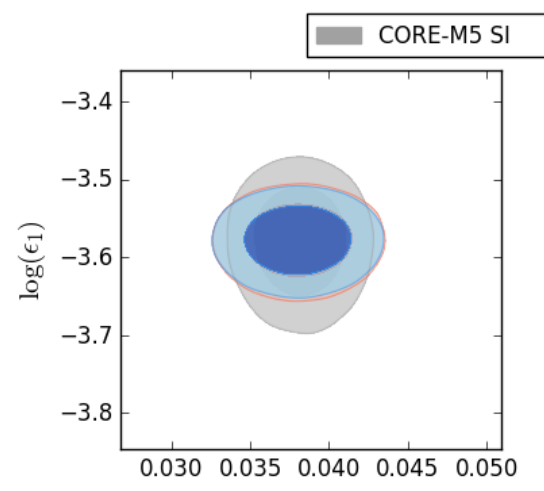

$\epsilon_{2}$

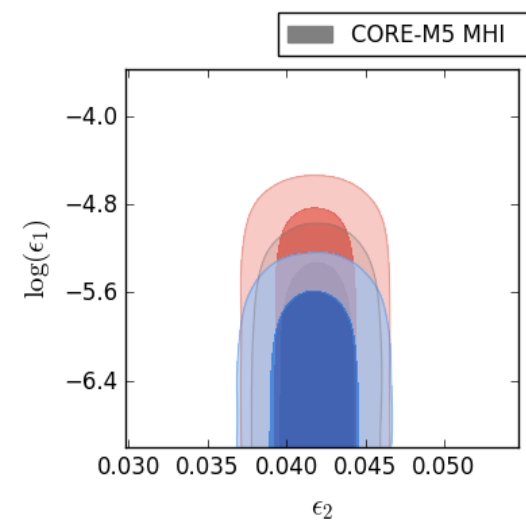

CORE-M5 SI Delensed $\ell>10 \square$ CORE-M5 SI Delensed

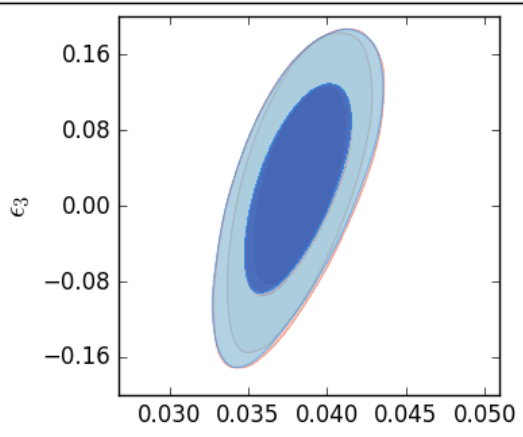

$\epsilon_{2}$

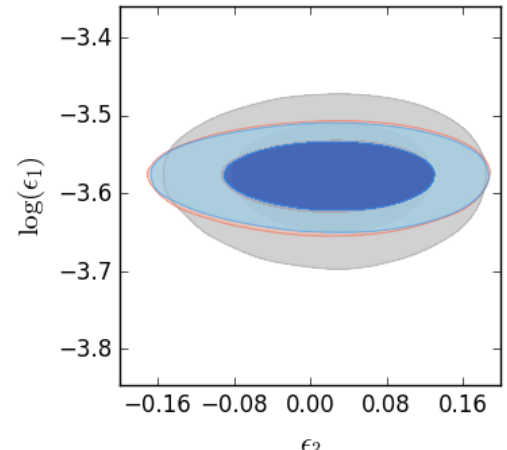

$\epsilon_{3}$

CORE-M5 MHI Delensed $\ell>10 \square$ CORE-M5 MHI Delensed
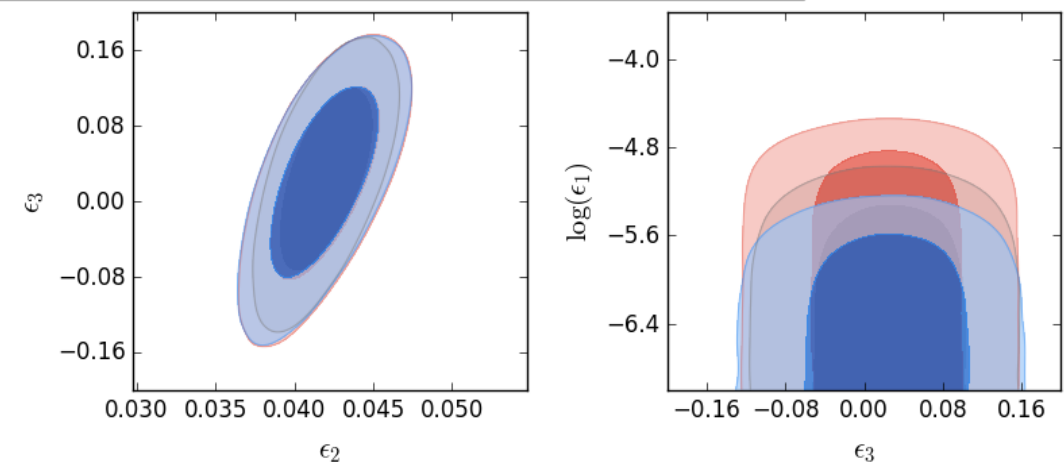

Figure 15: Forecasts on the Hubble flow parameters presented in Sec. 5.1.1 (blue), when the low multipoles $\ell<10$ are removed (red), and when delensing is not performed (grey), for CORE-M5 and SI (top panel) and MHI (bottom panel) as the fiducial model.

when the first multipoles $\ell<10$ are removed from the analysis and when delensing is not carried out. In Fig. 15, the 2D posterior contours on the Hubble flow parameters are displayed for COREM5 with and without $\ell<10$ and with and without delensing when SI and MHI are the fiducial models.

For SI, one can see that the constraints are almost unchanged when removing low multipoles, and slightly degraded without delensing. We found that this translates into no substantial difference for the constraints in the space of inflationary models and reheating expansion history (see Secs. 5.2 and 5.3). We therefore conclude that our analysis is robust under low multipoles removal and delensing in this case.

For MHI, however, the constraints on $\epsilon_{2}$ and $\epsilon_{3}$ do not change much when the low multipoles are removed, but $\epsilon_{1}$ is substantially affected. If delensing is not performed, the constraint on $\epsilon_{1}$ is weaker, but the main degradation comes from low multipole removal. We also found that in this case substantial differences for model comparison and reheating constraints are obtained.

\subsection{Inflationary model comparison}

We now investigate how the constraints of Sec. 5.1.1 translate into a Bayesian comparison of inflationary models. As mentioned above, Planck has allowed us to rule out one third of single- 

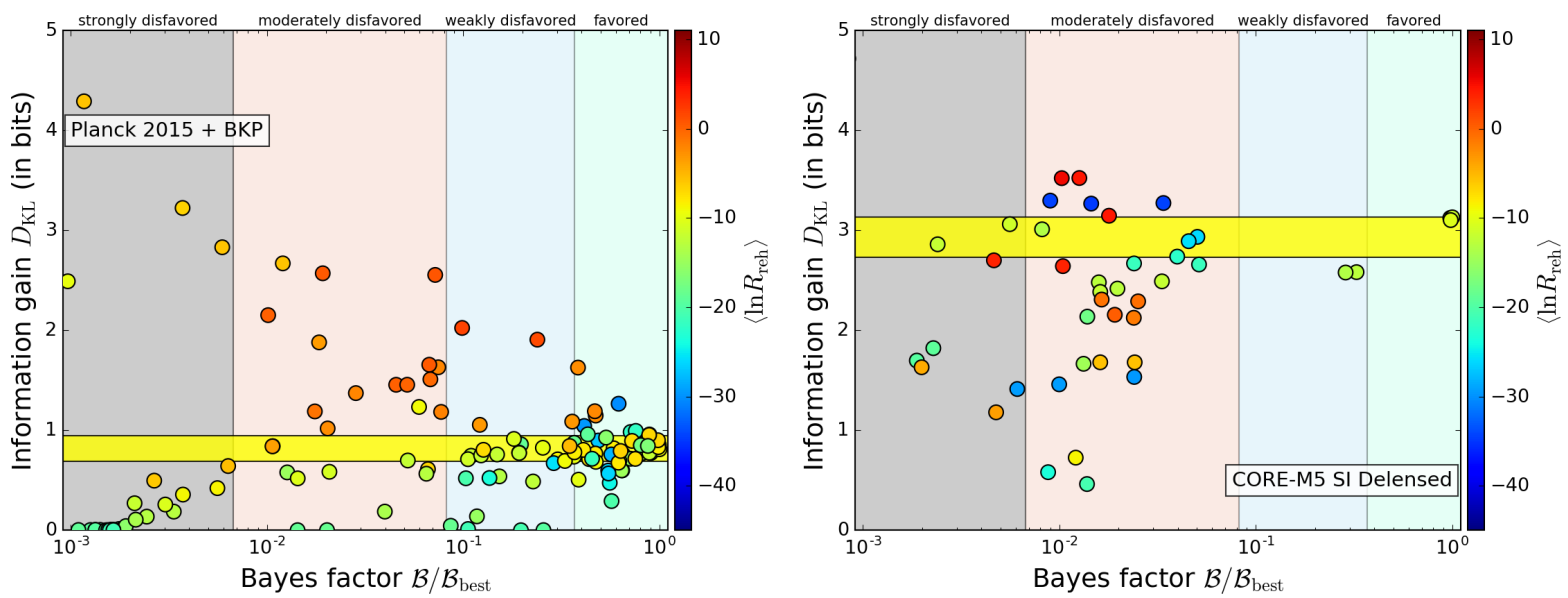

Figure 16: Information gain on reheating measured by the Kullback-Leibler divergence $D_{\mathrm{KL}}$ (in bits) on the reheating parameter $\ln R_{\text {reh }}$ for Planck $2015+$ BKP joint cross-correlation [54] (left panel, results taken from Ref. [228]) and for CORE-M5 if SI is the fiducial model, as a function of the Bayesian evidence normalized to the best model. Each circle represents one model and the $x$-axis is divided into Jeffreys categories. The circle color traces the mean value of the reheating parameter $\ln R_{\text {reh }}$. The yellow band represents the $1 \sigma$ deviation around the mean value for $D_{\mathrm{KL}}$.

field slow-roll models listed in Ref. [102], but still left us with about $27 \%$ of favored models. In this Section, we study how much this number could be decreased with a CORE-type mission.

In practice, we computed the Bayesian evidence [229-232] $\mathcal{E}$ of all inflationary models $\mathcal{M}_{i}$ listed in Encyclopaedia Inflationaris [102] using the priors proposed in Ref. [205]. We have followed the same method as described in Refs. [205, 233-237] which relies on the ASPIC library [238] and involves the derivation of an effective marginalized likelihood function depending only on the primordial parameters. All evidences have then been derived using the MULTINEST nested sampling algorithm $[180,181,239]$ with a target accuracy of $10^{-4}$ and the number of live points equal to 30000 .

Under the principle of indifference, two models $\mathcal{M}_{i}$ and $\mathcal{M}_{j}$ can be compared by computing the ratio $\mathcal{E}_{i} / \mathcal{E}_{j}$ of their Bayesian evidence. This ratio is called the Bayes factor and is displayed in Figs. 16 and 17 for various experimental setups (Planck LiteCORE-120, CORE-M5, and opt$\mathrm{COrE}+$ ) and for the two fiducial models SI and MHI considered in this work. Each model is represented with a circle and its position along the $x$-axis stands for its Bayes factor with the best model found in each case. The vertical colored stripes stand for Jeffreys empirical scale where if $\ln \left(\mathcal{E}_{i} / \mathcal{E}_{j}\right)>5, \mathcal{M}_{j}$ is said to be "strongly disfavored" with respect to $\mathcal{M}_{i}$, "moderately disfavored" if $2.5<\ln \left(\mathcal{E}_{i} / \mathcal{E}_{j}\right)<5$, "weakly disfavored" if $1<\ln \left(\mathcal{E}_{i} / \mathcal{E}_{j}\right)<2.5$, and the result is said to be "inconclusive" if $\left|\ln \left(\mathcal{E}_{i} / \mathcal{E}_{j}\right)\right|<1$.

Compared to Planck where 52 models are still favored, one can see that only a few models among those listed in Ref. [102] would remain favored if SI is taken as the fiducial model, and around 15 with MHI, corresponding to a large improvement of the constraints in model space. Interestingly though, the level of constraints increases only moderately when going from LiteCORE to COREM5 and optCOrE + . 


\section{$5.3 \quad$ Reheating}

Inflation is connected to the subsequent hot Big Bang phase through an era of reheating, during which the energy contained in the inflationary fields eventually decays into the Standard Model degrees of freedom. The amount of expansion during this epoch determines the amount of expansion between the Hubble crossing time of the physical scales probed in the CMB and the end of inflation [240-244]. As a consequence, the kinematics of reheating affects the time frame during which the fluctuations probed in cosmological experiments emerge, and hence the location of the observable window along the inflationary potential. This effect can be used to extract constraints on a certain combination $\ln R_{\text {reh }}$ of the averaged equation-of-state parameter $\bar{w}_{\text {reh }}$ during reheating and the energy density at the end of reheating $\rho_{\text {reh }}$,

$$
\ln R_{\mathrm{reh}}=\frac{1-3 \bar{w}_{\mathrm{reh}}}{12\left(1+\bar{w}_{\mathrm{reh}}\right)} \ln \left(\frac{\rho_{\mathrm{reh}}}{\rho_{\mathrm{end}}}\right)+\frac{1}{4} \ln \left(\frac{\rho_{\mathrm{end}}}{M_{\mathrm{Pl}}^{4}}\right)
$$

where $\rho_{\text {end }}$ is the energy density at the end of inflation. Around 0.8 bits of information on this quantity can be extracted $[228,245]$ from the latest CMB measurements. However, it is still at a level where if the averaged equation-of-state parameter during reheating takes a fixed value that is not too close to $-1 / 3$ (for instance, vanishes), the reheating temperature cannot be constrained very well.

The amount of information gained regarding reheating can therefore be quantified using the Kullback-Leibler divergence [246] between the prior distribution $\pi$ and the posterior $P$ on $\ln R_{\text {reh }}$,

$$
D_{\mathrm{KL}}=\int P\left(\ln R_{\mathrm{reh}} \mid D\right) \ln \left[\frac{P\left(\ln R_{\mathrm{reh}} \mid D\right)}{\pi\left(\ln R_{\mathrm{reh}}\right)}\right] \mathrm{d} \ln R_{\mathrm{reh}},
$$

which is a measure of the amount of information provided by the data $D$ about $\ln R_{\text {reh }}[247,248]$. This quantity is also the discrepancy measure between the posterior $P$ and the prior $\pi$ when the prior is viewed as an approximation of the posterior. Because the Kullback-Leibler divergence is invariant under any reparametrizations $x=f\left(\ln R_{\text {reh }}\right)$ and uses a logarithmic score function as in the Shannon's entropy, it is a well-behaved measure of information [249]. In Fig. 16, the KullbackLeibler divergence of all single-field models within Encyclopaedia Inflationaris [102] are displayed as a function of their Bayesian evidence for Planck 2015 plus BKP joint cross-correlation [54] and CORE-M5 if SI is the fiducial model. One can see that on average one would go from 0.8 bit of information about reheating to more than 3 bits. Let us recall that 1 bit is the amount of information contained in answering "yes" or "no" to a given question. In the present case, the question is about the values of $\ln R_{\mathrm{reh}}$, and the current CMB data answer on average whether $\ln R_{\text {reh }}$ is large or small. In the same manner, 2 bits would correspond to choosing between 4 possible values for $D_{\mathrm{KL}}$ and 3 bits to choosing between 8 possible values. Going from 1 to 3 bits is therefore a considerable gain that would open a new observational window into the physics of the end of inflation [250-252]. In Fig. 17, the same results are displayed for LiteCORE-120, CORE-M5, and optCOrE + when SI and MHI are used as fiducial models. One can see that even if $r$ is not detected (here described by the case where the fiducial model is MHI), more than 2 bits of information would still be gained. 

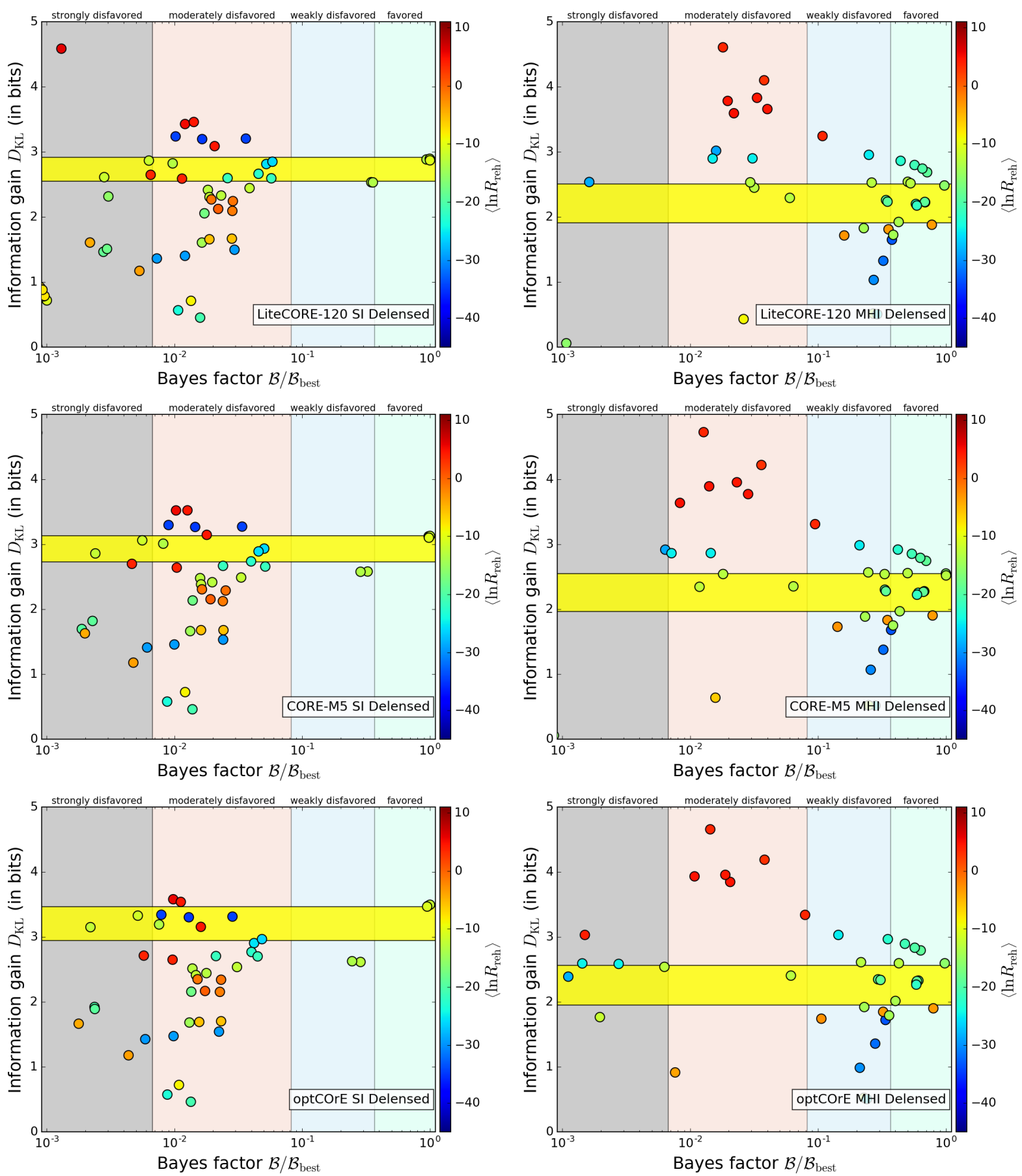

Figure 17: Information gain on reheating measured by the Kullback-Leibler divergence $D_{\mathrm{KL}}$ (in bits) on the reheating parameter $\ln R_{\text {reh }}$ for LiteCORE-120 (top panels), CORE-M5 (middle panels) and optCOrE + (bottom panels), for SI (left panels) and MHI (right panels) as fiducial models as a function of the Bayesian evidence normalized to the best model. Each circle represents one model and the $x$-axis is divided into Jeffreys categories. The circle color traces the mean value of the reheating parameter $\ln R_{\text {reh }}$. The yellow band represents the $1 \sigma$ deviation around the mean value for $D_{\mathrm{KL}}$. With CORE-type missions, only a few models would remain favored (compared to 52 currently with Planck) and one would gain between 2 and 3 bits of information about reheating on average (compared to 0.8 with Planck). 


\subsection{Summary}

The analysis in this Section demonstrates the potential of CORE to discriminate among different inflationary models. Considering SI as the reference model, one ends up with only 3 favored and 2 weakly disfavored models among those listed in Ref. [102]. For the MHI reference model, which would correspond to an upper bound on primordial B-modes, we find $\sim 10$ favored and $\sim 15$ weakly disfavored models, always among those listed in Ref. [102].

More than the angular resolution of the apparatus (linked to the mirror size), we have shown that the sensitivity of the instrument plays the most important role in improving the forecasts on $\epsilon_{1}$, and thus on $r$. This is particularly important for the information gain on reheating which increases from less than 1 bit with Planck to more than 3 bits with CORE if SI is taken as the fiducial model, and more than 2 bits if MHI is the fiducial model.

These results can be summarized by Fig. 18, displaying the Planck CORE-M5 and optCOrE + posteriors in the $\left(\epsilon_{1}, \epsilon_{2}\right)$ plane, considering SI or MHI as the reference model. Not only would CORE allow us to bring the number of favored inflationary models listed in Ref. [102] down to a few, but it would even distinguish among different reheating scenarios. Interestingly, this could allow us to distinguish models that share nearly the same inflationary potential such as Higgs Inflation and the Starobinsky model, but could have different reheating temperature under

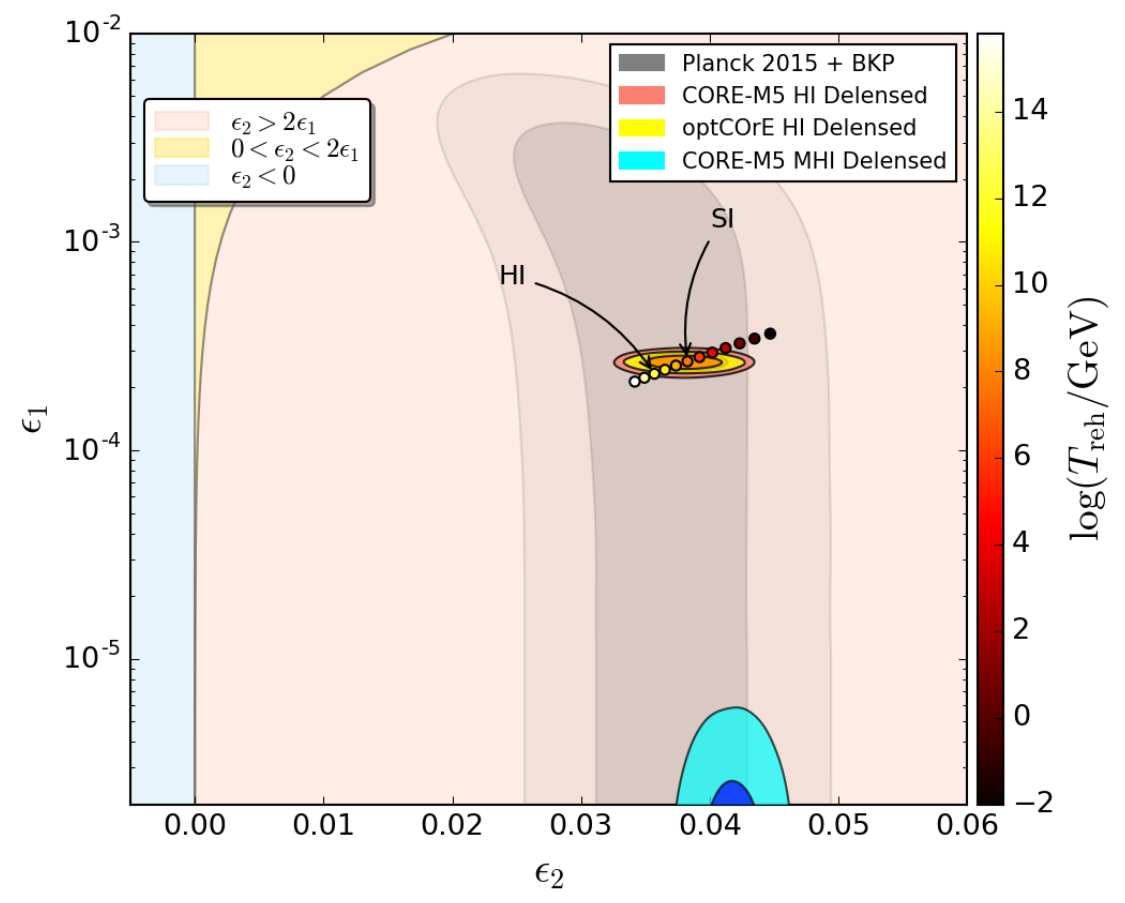

Figure 18: Constraints in the $\epsilon_{1}-\epsilon_{2}$ plane ( $1 \sigma$ and $2 \sigma$ contours) for Planck 2015 plus the BKP joint cross-correlation, CORE-M5 and optCOrE + (with SI and MHI as fiducial models). The predictions of the model SI have been displayed assuming $\bar{w}_{\text {reh }}=0$, where the color encodes the reheating temperature $T_{\text {reh }}$. The models indicated as HI (Higgs Inflation) and SI (Starobinsky Inflation) share the same inflationary potential, but are endowed with different reheating temperatures (around $10^{12} \mathrm{GeV}$ for $\mathrm{HI}$ and $10^{8} \mathrm{GeV}$ for SI), which a CORE-type mission could distinguish at $2 \sigma$. 


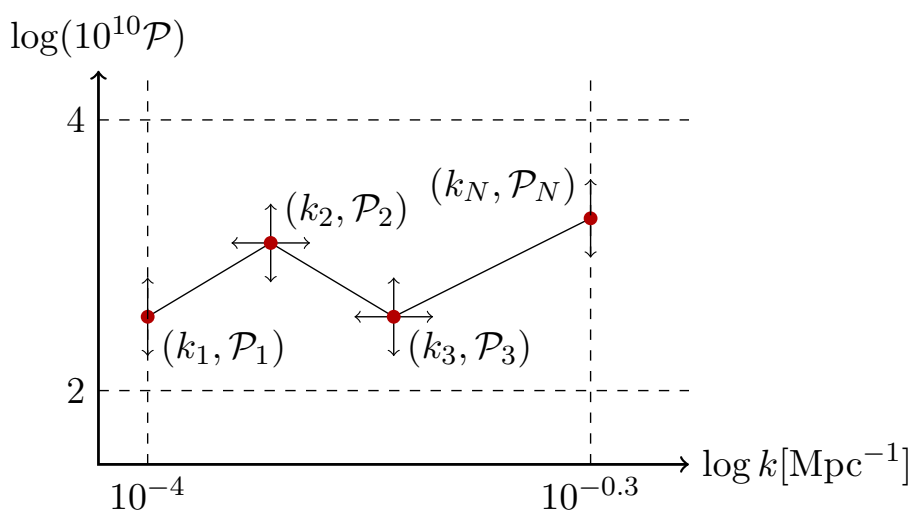

Figure 19: The scalar primordial power spectra are reconstructed using $N$-point interpolating logarithmic splines. The positions of the points in the $(k, \mathcal{P})$ plane are treated as likelihood parameters with log-uniform priors. Further, the $k$-positions sorted a priori such that $k_{1}<k_{2}<\cdots<k_{N}$ with $k_{1}$ and $k_{N}$ fixed. Units of $k$ are inverse megaparsecs.

minimal assumptions on the inflaton coupling [253]. This opens fascinating prospects to better understand the physics of the very early universe.

\section{Testing deviations from a power law spectrum}

The fundamental contact of inflationary theory with the observations comes from comparing the model predictions for the primordial scalar $\mathcal{P}_{\mathcal{R}}$ and tensor $\mathcal{P}_{\mathcal{T}}$ power spectra with the data. These are processed by cosmological transfer functions to yield predictions of CMB temperature and polarization multipoles $C_{\ell}$. Comparing the theoretical $C_{\ell}$ 's with the observed values yields constraints on the inflationary model parameters.

In this Section we reverse this procedure and reconstruct the primordial power spectra directly from the data. This procedure has been successfully applied to the Planck 2015 data [5] for the scalar primordial power spectrum using the methods of Gauthier and Bucher [254], Vazquez et. al. [255], Aslanyan et al. [256] and the methods of Bond et al. [5].

\subsection{Primordial power spectra reconstruction methodology}

To reconstruct primordial power spectra, we follow the methodology of Section 8.2 of the Planck inflation paper [5]. Instead of using the traditional amplitude-tilt parameterization $\left(A_{\mathrm{s}}, n_{\mathrm{s}}\right)$, we instead use an $N$-point interpolating logarithmic spline (Fig. 19), with the positions of the knots considered as free parameters in the full posterior distribution.

We compute posteriors (conditioned on $N$ ) using the PolyChord nested sampler [257, 258], also varying the cosmological and any nuisance parameters. We then use evidence values for each model to correctly marginalize out the number of knots $N$.

To plot our reconstructions of the power spectra, we compute the marginalized posterior distribution of $\log \mathcal{P}$ conditioned on $k$. The iso-probability confidence intervals are then plotted in the $k$ - $\mathcal{P}$ plane (e.g., Fig. 20).

To quantify the constraining power of a given experiment, we use the conditional KullbackLeibler (KL) divergence [259], defined in Eq. 5.3. For our reconstructions, we compute the KL 

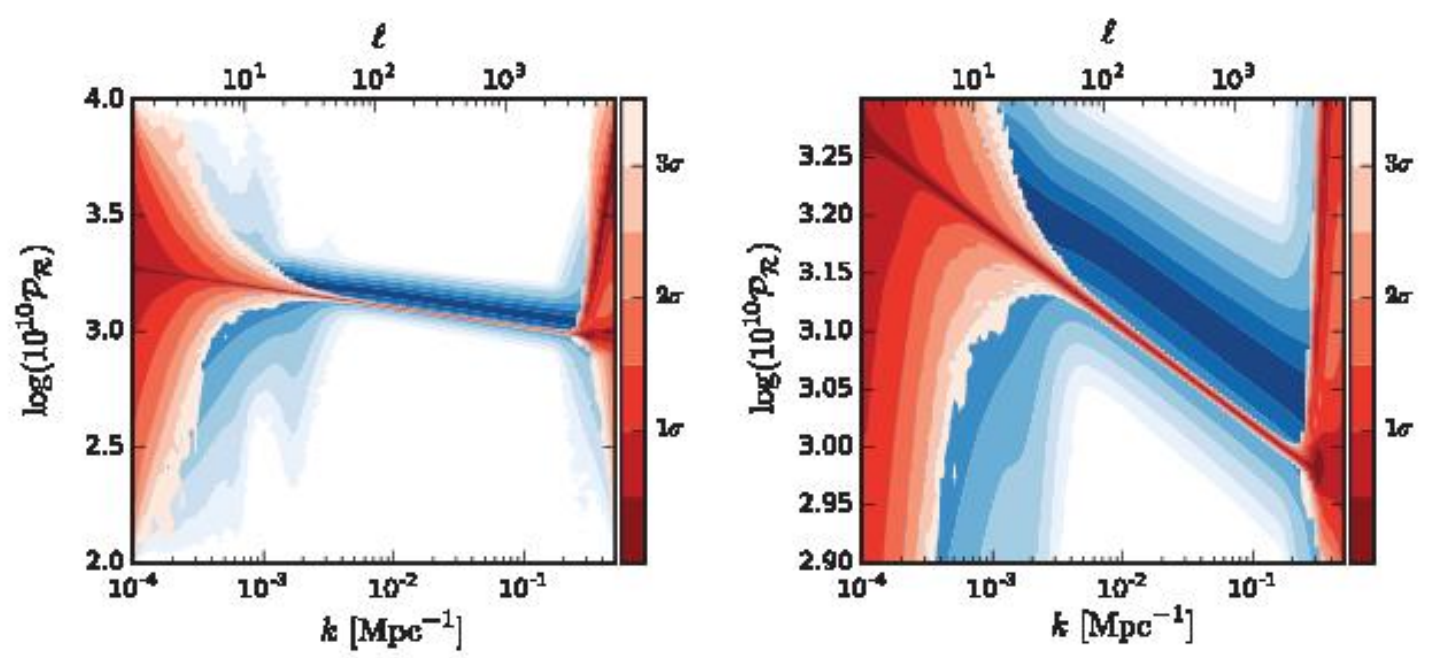

Figure 20: Left: Reconstruction of a simulated featureless scalar power spectrum for a CORE-M5 experiment (in red), compared to existing constraints provided by Planck (in blue). Right: Zoomed-in version of the left figure to show the order of magnitude increase in constraining power that would be provided by CORE.

divergence of each distribution conditioned on $k$ and $N$, and then marginalize over $N$ using evidences to produce a $k$-dependent number which quantifies the logarithmic compression (i.e., information) that each experiment provides at each value of $k$ (e.g., Fig. 21).

This reconstruction approach has the advantage of being somewhat agnostic to any specific inflation model and constitutes a 'blind' reconstruction of the power spectra. One could also search for parameterized features as discussed in Section 9 of the Planck inflation paper [5]. In this Section we shall focus on answering the question as to how well a blind reconstruction could pick up a variety of features using CORE-M5 data resolution. This approach is by no means unique. Other authors have used a binned spectrum [260], cubic splines [261] and cross-validation [262], as well as reconstructions motivated by phenomenology [263] or inflationary models [264, 265]. The approach chosen for this Section is motivated more by its conceptual simplicity than anything else.

\subsection{Featureless scalar power spectrum}

To quantify the basic constraining power of the experiments detailed in Tables 2 and 3, we begin by examining how well these experiments could constrain a featureless scalar power spectrum. Simulated likelihoods are generated from a featureless tilted power spectrum with $n_{\mathrm{S}}=0.96$ and $A_{\mathrm{s}}=2.1 \times 10^{-9}$ with $T T, T E$, and $E E$ data for COrE + , LiteCORE-120, LiteCORE-80, LiteBIRD, and CORE-M5 experiments.

Figure 20 shows the marginalized posterior distribution for the CORE-M5 experiment. As usual, the reconstructions demonstrate the lack of reconstructive power at low $k$ due to cosmic variance and the inability to reconstruct above the resolving power of $\ell \sim 3000$. CORE-M5 provides an order of magnitude better constraint on the amplitude of the power spectrum relative to the Planck 2015 data (shown for reference in blue). 


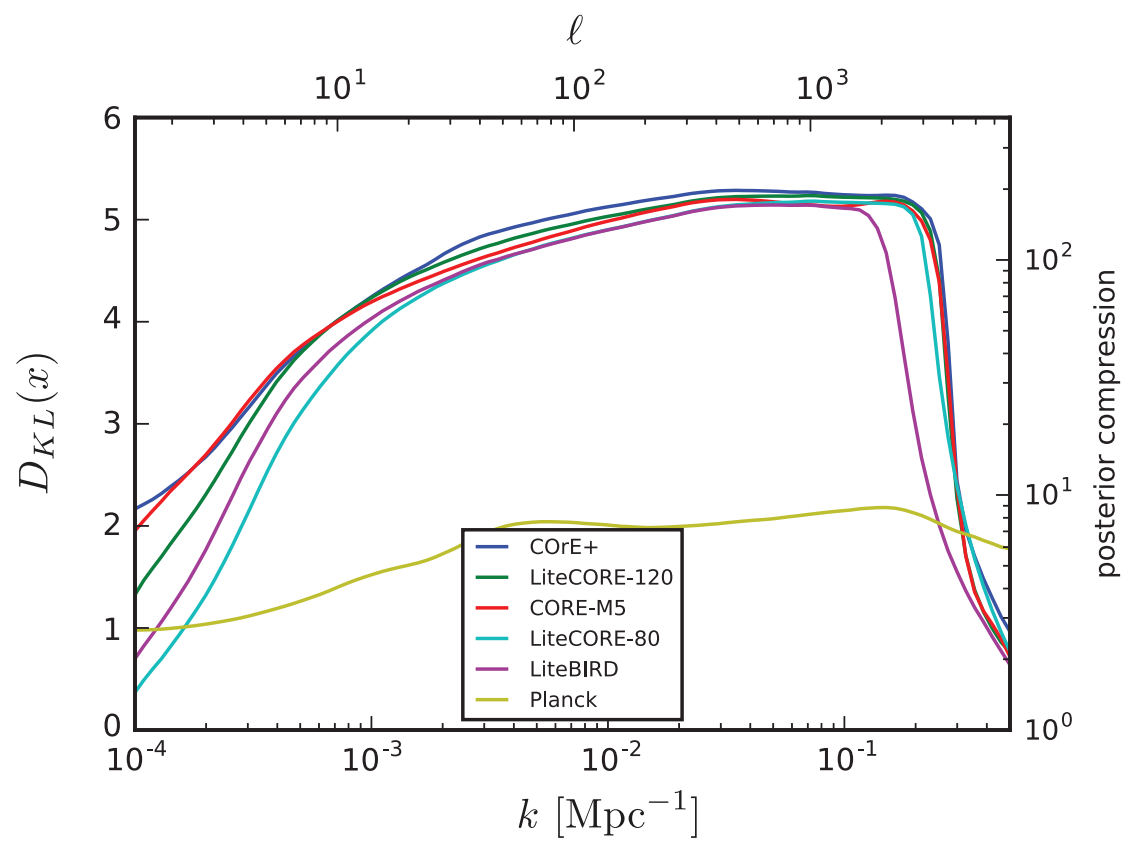

Figure 21: The amount of information that CORE experiments would provide on the scalar primordial power spectrum. The Kullback-Leibler divergence shows that all configurations of the experiment provide a similar level of information. As expected, the prior-to-posterior compression drops off at low $\ell$ due to cosmic variance, and high $\ell$ at the limits of the experiment. All CORE configurations thus provide an order of magnitude more information than Planck 2015. It is worth remarking that we use Planck 2015 real data here. The improvement of the CORE experiments with respect to Planck 2015 real data is largely related to improved determination of $A_{s}$, also connected to the cosmic variance limited measurement of $\tau$.

To quantify the level of this increase in information, Fig. 21 shows the information gain for each of these experiments at each value of $k$ in comparison with Planck. One can see that all three configurations provide similar levels of information on the primordial power spectrum, and an order of magnitude more information than Planck, with LiteBIRD and LiteCORE-80 failing to provide information above $\ell \sim 1350$ and 2400 , respectively.

The mock likelihoods used throughout this Section follow the methodology detailed in Section 3.1, where the fiducial "observed" likelihoods $\hat{\mathbf{C}}_{\ell}$ are computed using CAMB. A more powerful analysis has also been employed which incorporates cosmic variance to add "scatter" to these fiducial $\hat{\mathbf{C}}_{\ell}$. The results remain quantitatively unchanged for $\ell>10$, both in $D_{K L}$ divergence (Fig. 21) and predictive posterior plots (Figs. 20, 22, 23, and 24). For $\ell<10$, noise features appear in higher $N$ predictive posteriors, but these are suppressed by correspondingly low evidence values. Quantitatively, the $D_{K L}$ constraining power is somewhat overestimated by using unscattered $\hat{\mathbf{C}}_{\ell}$ for these extremely low values of $\ell$. We have chosen to present the unscattered fiducial versions of our plots for simplicity and consistency with the rest of this paper.

\subsection{Scalar power spectrum with wiggles and cutoffs}

With such strong constraining power, any of these CORE experiments would be able to detect features in the primordial power spectrum that are currently unresolved using Planck data. To show this, we produced a likelihood for a wiggly power spectrum as would be generated by an 

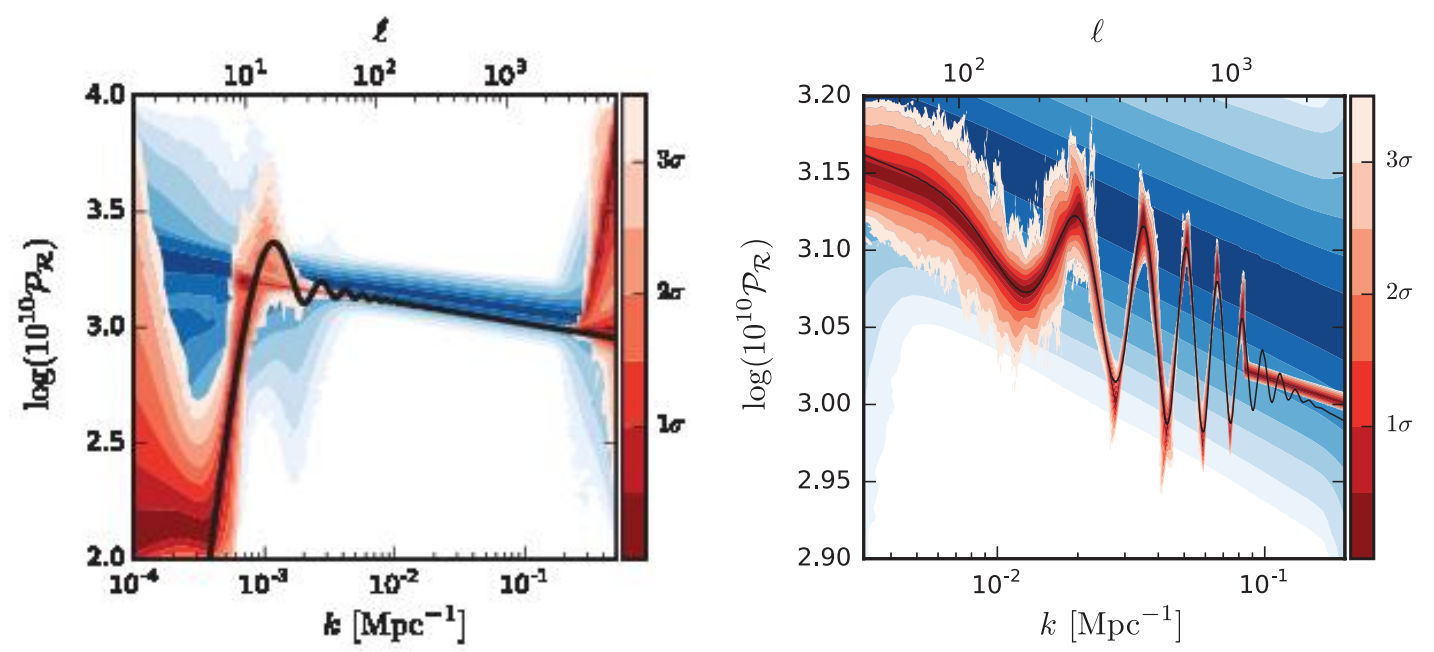

Figure 22: As in Fig. 20, but now reconstructing a simulated power spectrum with features (black lines). Left: Cut-off as would be generated by a brief period of fast-roll expansion prior to slow roll inflation. The additional constraining power provided by CORE would allow detection of low $\ell$ features such as cutoffs and wiggles. Right: Reconstruction of higher- $\ell$ linearly-sinusoidal wiggles generated by a reduction in the speed of sound of the inflaton, as described in Section 2.3.3. In this last case, the reconstruction only picks up the feature when the prior on the nodes' positions is restricted to the region where the feature is active; the smaller-scale wiggles prove to be harder to reconstruct.
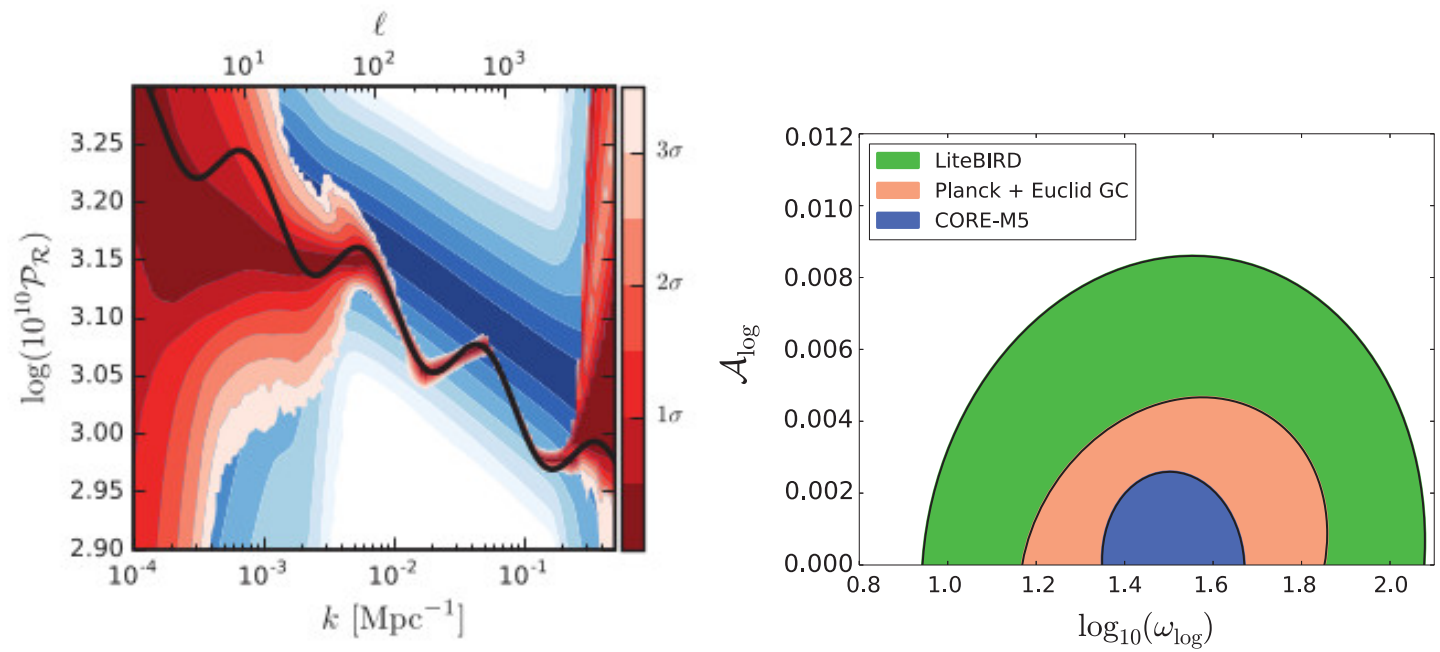

Figure 23: Left: As in Fig. 20, but now reconstructing a simulated power spectrum with low frequency sinusoidal logarithmic oscillations (black line), with $A_{\log }=0.03, \omega_{\log }=3$ and $\psi_{\log }=0$. Right: constraints on superimposed sinusoidal logarithmic oscillations with an higher frequency, comparable to those providing a best-fit to Planck 2015 data [5]. Whereas a blind reconstruction technique is unsuitable for high frequency oscillations, CORE-M5 performs better than LiteBIRD or Planck in combination with Euclid spectroscopic galaxy clustering for this type of parameterized features. Contours indicate $68 \%$ confidence intervals. 


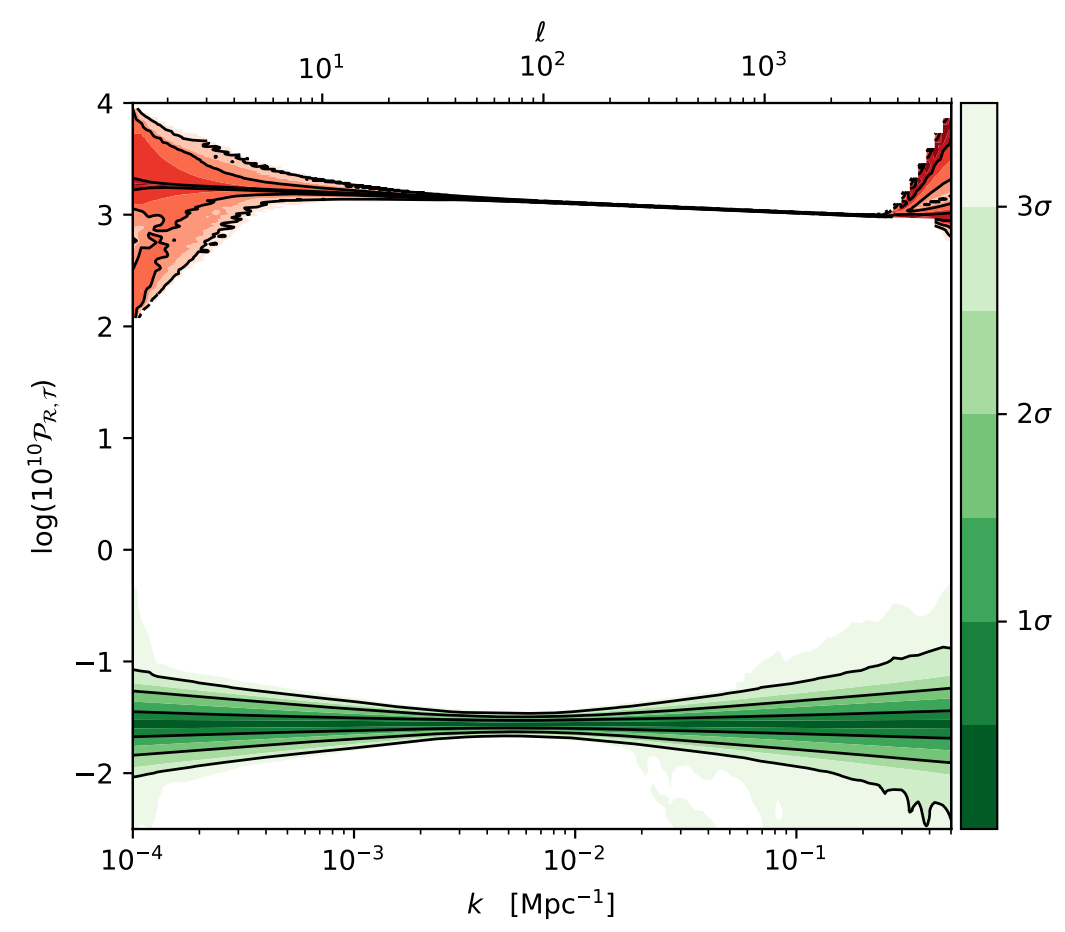

Figure 24: Simultaneous reconstructions of the tensor (lower, green) and scalar (upper, red) power spectra for CORE-M5 forecast if $r=0.01$.

epoch of fast-roll inflation prior to slow roll [266]. The left hand plot of Fig. 22 shows that COREM5 has the capacity to blindly reconstruct a cutoff and the first oscillation which could be hidden beneath Planck 2015 resolution. This remains true even when cosmic variance noise is added.

CORE experiments would also be capable of reconstructing smaller localized features, such as those produced by reduced adiabatic sound speeds (Section 2.3.3), as demonstrated in Fig. 22.

\subsection{Superimposed logarithmic oscillations}

Resonant models including periodic oscillations in the potential lead to superimposed sinusoidal oscillation in the spectrum and bispectrum [148], as discussed in Section 2. The periodic oscillations appearing in axion monodromy [74, 75] belong to this general class of models [267]. We consider the phenomenological parameterization

$$
\mathcal{P}_{\mathcal{R}}(k)=A_{\mathrm{s}}\left(\frac{k}{k_{*}}\right)^{n_{\mathrm{s}}-1}\left(1+A_{\log } \sin \left[\omega_{\log } k+\psi_{\log }\right]\right) .
$$

As shown in Fig. 23, CORE-M5 also has the resolving power to blindly reconstruct logarithmic oscillations with an amplitude at a percent level for low frequencies, i.e., $\omega_{\log }=3$. For higher frequencies, the blind reconstruction scheme would require prohibitively large numbers of knots. Nevertheless, as is shown in the right-hand panel of Fig. 23, CORE-M5 is still capable of extracting information about such models by directly constraining the parameters of the superimposed oscillations in Eq. (6.1). Further detail on the Fisher forecast methodology for this approach is provided by [268]. 


\subsection{Reconstructing the tensor power spectrum}

Finally, we demonstrate that if there is a detectable scalar-to-tensor ratio, CORE-M5 would also be able to reconstruct this spectrum. Fig. 24 shows a simultaneous reconstruction of both the scalar and tensor power spectrum, where we use two independent reconstructions on simulated data with a flat tensor power spectrum for $r=0.01$.

\section{Testing the adiabaticity of initial conditions: constraining isocurvature}

In the simplest single-field inflationary models only the adiabatic growing mode is excited. Indeed, the six-parameter concordance cosmological model, which was found to provide an adequate fit to the WMAP and Planck data, includes only the growing adiabatic mode, but this does not necessarily mean that other modes could not have been excited. Much theoretical work has been devoted to studying multi-field inflationary models and other extended inflationary models, and many of these models predict that isocurvature modes could have been excited. It is therefore of great interest to test the hypothesis of adiabaticity by searching for isocurvature modes. At one time, proposals were put forth in which isocurvature modes would offer an alternative to adiabatic modes for the formation of structure and for imprinting the CMB anisotropies. But it soon became apparent that such models in which the growing adiabatic mode was not excited were not viable. As a consequence the emphasis shifted to exploring scenarios in which both adiabatic and isocurvature modes were excited, possibly in a correlated manner [269-272]. The discussion below explores how CORE and other future satellite configurations will be able to improve on the constraints already established by Planck in Refs. [3, 5]. See also references therein for a more comprehensive list of literature regarding the isocurvature modes.

We study a model where the primordial curvature perturbation, or the adiabatic mode, is correlated with a primordial Cold dark matter Density Isocurvature (CDI) mode. ${ }^{11}$ In our analysis we use the MultiNest nested sampling algorithm and a modified version of CosmoMC and CAMB capable of calculating theoretical predictions for the arbitrarily correlated adiabatic and isocurvature modes and simultaneously including primordial tensor perturbations in a consistent way.

\footnotetext{
${ }^{11}$ Here we do not consider neutrino isocurvature modes. In addition to the recent Planck results [3, 5], observational work on the neutrino modes include [273-275] using the WMAP data. Furthermore, we restrict the analysis to the power spectrum level where the baryon density isocurvature (BDI) mode or the total matter density isocurvature (simultaneously $\mathrm{CDI}$ and BDI) can be mapped into $\mathcal{I}_{\mathrm{CDI}}^{\text {effective }}=\mathcal{I}_{\mathrm{CDI}}+\left(\Omega_{\mathrm{b}} / \Omega_{\mathrm{c}}\right) \mathcal{I}_{\mathrm{BDI}}$. The trispectrum may be useful for distinguishing between CDI and BDI [276], but we leave these forecasts to future work. In the case of an exact cancellation between $\mathcal{I}_{\mathrm{CDI}}$ and $\left(\Omega_{\mathrm{b}} / \Omega_{\mathrm{c}}\right) \mathcal{I}_{\mathrm{BDI}}$, we have $\mathcal{I}_{\mathrm{CDI}}^{\text {effective }}=0$, so there is no isocurvature perturbation between the radiation and the matter. These are called compensated isocurvature perturbations (CIP), and because baryons behave differently from dark matter on small scales, CIPs modify the angular power spectrum compared to the pure adiabatic prediction, but these scales correspond to multipoles $\ell \gtrsim 10^{5}-10^{6}$ [277]. At much larger scales, CIPs may imprint a lensing-like signal onto the angular power spectrum at second order in the CIP amplitude $\Delta_{\text {rms }}$ [278]. Using adiabatic $\Lambda$ CDM fiducial TT, TE, and EE data, we find $\Delta_{\text {rms }}^{2}<0.0019$ at $95 \%$ CL for CORE-M5. LiteBIRD gives slightly weaker constraints than Planck, whereas CORE-M5 gives three times stronger constraints and is close to the cosmic variance limit. Compared to the future ground-based instruments [68] CORE-M5 performs about a factor of two better. More detailed power spectrum based forecasts for CIP are presented in [279].
} 


\subsection{The model and its parameterization}

The full details of the parameterization and notation including various symbols appearing in the Figures are explained in $[3,5]$. Here we summarize the main assumptions and choices. The model has the usual 4 background parameters of the flat $\Lambda$ CDM model $\left(\Omega_{\mathrm{b}} h^{2}, \Omega_{\mathrm{c}} h^{2}, \theta_{\mathrm{MC}}, \tau\right)$ and two parameters describing the assumed power law primordial curvature perturbation power $\mathcal{P}_{\mathcal{R} \mathcal{R}}(k)$. In addition we have two parameters that describe a power law primordial isocurvature perturbation spectrum $\mathcal{P}_{\mathcal{I I}}(k)$ and one parameter that describes the correlation amplitude between the curvature and isocurvature perturbations $\mathcal{P}_{\mathcal{R} \mathcal{I}}\left(k_{0}\right)$ at a pivot scale corresponding to $k_{0}$. The curvature perturbation has a spectral index $n_{\mathcal{R} \mathcal{R}}$ (called $n_{\mathrm{S}}$ in the other Sections), and the isocurvature perturbation has an independent spectral index $n_{\mathcal{I I}}$. The correlation power does not have an independent spectral index in our model, but is simply a power law with index $n_{\mathcal{R} \mathcal{I}}=\left(n_{\mathcal{R} \mathcal{R}}+n_{\mathcal{I I}}\right) / 2$. This restriction means that the correlation fraction

$$
\cos \Delta \equiv \frac{\mathcal{P}_{\mathcal{R I}}(k)}{\sqrt{\mathcal{P}_{\mathcal{R} \mathcal{R}}(k) \mathcal{P}_{\mathcal{I I}}(k)}} \in[-1,+1]
$$

is constant with respect to $k$ (i.e., scale independent). If the correlation spectral index $n_{\mathcal{R} \mathcal{I}}$ were allowed as an independent parameter, the correlation fraction would not remain between -1 and +1 over all scales.

Spectral indices are not suitable for describing this extended model, since the pure adiabatic $\Lambda \mathrm{CDM}$ model should be represented by a single set of values (one point) in the parameter space of the extended model. However if we chose the isocurvature power at a pivot scale $k_{0}$, [i.e., $\left.\mathcal{P}_{\mathcal{I I}}\left(k_{0}\right)\right]$ as a primary isocurvature parameter of our model and for the other primary parameter the spectral index $n_{\mathcal{I I}}$, the pure adiabatic model would be described by a line where $\mathcal{P}_{\mathcal{I I}}\left(k_{0}\right)=0$, but $n_{\mathcal{I I}}$ can take any value between $-\infty$ and $+\infty$. As a consequence, the larger prior range we allowed for $n_{\mathcal{I I}}$, the more biased our results would be toward the pure adiabatic model, since the marginalization over $n_{\mathcal{I I}}$ direction with nearly zero $\mathcal{P}_{\mathcal{I I}}\left(k_{0}\right)$ would artificially increase the weight of the nearly adiabatic models $\left(\mathcal{P}_{\mathcal{I I}}\left(k_{0}\right) \approx 0\right.$ ) by a factor $\Delta n_{\mathcal{I I}}$, (i.e., by the prior width of the $n_{\mathcal{I I}}$ parameter).

A solution to this problem was proposed in Ref. [280], and first applied to isocurvature analysis in Ref. [281] by the BOOMERANG team and in Ref. [282] using in addition the WMAP 3-year and ACBAR data. Also in the Planck 2013 [3] and 2015 [5] papers, the same approach was adopted, in 2015 both for isocurvature and tensor perturbations. We parameterize the primordial power law perturbations by specifying their amplitudes at two different scales: at a large scale corresponding to a small wave number $k=k_{1}=k_{\text {low }}=0.002 \mathrm{Mpc}^{-1}$, and at a small scale corresponding to a large wave number $k=k_{2}=k_{\text {high }}=0.100 \mathrm{Mpc}^{-1}$. Thus the primary parameters describing the primordial perturbations are $\mathcal{P}_{\mathcal{R} \mathcal{R}}\left(k_{1}\right), \mathcal{P}_{\mathcal{R} \mathcal{R}}\left(k_{2}\right), \mathcal{P}_{\mathcal{I I}}\left(k_{1}\right), \mathcal{P}_{\mathcal{I I}}\left(k_{2}\right)$, and $\mathcal{P}_{\mathcal{R I}}\left(k_{1}\right)$. The sign convention is such that a positive $\mathcal{P}_{\mathcal{R} \mathcal{I}}$ leads to extra power at low multipoles in the temperature angular power and a negative primordial correlation leads to a negative observational contribution [see also footnote 12 on page 58].

The spectral indices are derived parameters calculated from the primary parameters [e.g., $\left.n_{\mathcal{I I}}=\ln \left(\mathcal{P}_{\mathcal{I I}}\left(k_{2}\right) / \mathcal{P}_{\mathcal{I I}}\left(k_{1}\right)\right) / \ln \left(k_{2} / k_{1}\right)+1\right]$. The parameter $\mathcal{P}_{\mathcal{R} \mathcal{I}}\left(k_{2}\right)$ is also a derived parameter. 
Other interesting derived parameters are the primordial isocurvature fraction

$$
\beta_{\text {iso }}(k)=\frac{\mathcal{P}_{\mathcal{I I}}(k)}{\mathcal{P}_{\mathcal{R} \mathcal{R}}(k)+\mathcal{P}_{\mathcal{I I}}(k)}
$$

and the correlation fraction $\cos \Delta$ defined in Eq. (7.1).

\subsection{Adiabatic fiducial data}

\subsubsection{Fitting a generally correlated mixture of adiabatic and CDI modes}

Figure 25 presents the constraints on primordial curvature, isocurvature, and correlation powers if the true primordial perturbations were purely adiabatic without a tensor contribution. We fit an extension of the standard $\Lambda \mathrm{CDM}$ model with three isocurvature parameters to these data. All datasets use TT, TE, and EE, but for CORE-M5 and COrE + we also give the results with the CMB lensing potential (PP) data included. BB and delensing analysis is not applied in this Subsection. In addition to forecasts for 'future experiments,' for comparison we show the constraints for simulated Planck TT, TE, EE data assuming the bluebook [283] white noise sensitivities divided by $\sqrt{2}$ using the 100,143, and $217 \mathrm{GHz}$ channels in inverse noise weighting (the factor $1 / \sqrt{2}$ takes into account that Planck operated at least twice as long as the nominal mission described in the bluebook). Finally, we make a MultiNest run with zero instrumental noise using the multipole range $\ell=2-3000$ (called "Ideal TT,TE,EE $\ell_{\max }=3000$ " in Fig. 25). The only 'noise' in this run is cosmic variance. Consequently, this represents an ideal case for TT, TE, EE.

The first panel of Fig. 25 provides a convenient way to represent the determination accuracy of the adiabatic scalar perturbations instead of the usual $\left(A_{\mathrm{S}}, n_{\mathrm{S}}\right)$ pair. We see directly the primordial curvature perturbation amplitude at large and small scales, denoted by the superscripts (1) and (2), respectively. The future missions (except LiteBIRD owing to its coarse angular resolution) constrain these parameters much better than Planck. LiteCORE-120, CORE-M5, and $\mathrm{COrE}+$ virtually reach the cosmic variance limit, and with PP included actually determine the curvature perturbation power better than the ideal cosmic variance limited experiment only with TT, TE, and EE.

For isocurvature, the second panel of Fig. 25 shows the key result. The point $(0,0)$ represents the pure adiabatic model. We notice a region which is allowed by both the real [5] and simulated Planck data, but which could be excluded by any of the future missions. For example, the primordial isocurvature powers $\mathcal{P}_{\mathcal{I I}}\left(k_{1}\right)=0.3 \times 10^{-10}$ and $\mathcal{P}_{\mathcal{I I}}\left(k_{2}\right)=2.3 \times 10^{-10}$ (corresponding to $\beta_{\text {iso }}\left(k_{1}\right) \approx 0.0125$ and $\beta_{\text {iso }}\left(k_{2}\right) \approx 0.1$ ) would not have been detected by Planck, but the future missions would be able to make a detection. The improvement compared to Planck on the upper bounds on isocurvature or correlation power are mostly in the parameters of small scales, labelled with the superscript (2), and are about a factor of two to three better. Also for the isocurvature parameters, all future configurations are very nearly at the cosmic variance (ideal) limit, except for LiteBIRD, which is clearly worse for most parameters.

As shown in Table 12, CORE-M5 can improve the upper bound on the primordial isocurvature fraction by a factor of 1.3 on large scales and 2.4 on small scales compared to the constraints from simulated Planck TT, TE, EE data. The tightest constraints on the isocurvature fraction occur at

scales in the middle of the $k$ or $\ell$ range probed, where $k_{\text {mid }}=0.05 \mathrm{Mpc}^{-1}$. Here the improvement over Planck is by a factor of 2.3. Again, in terms of these parameters, all the future configurations 
(a)
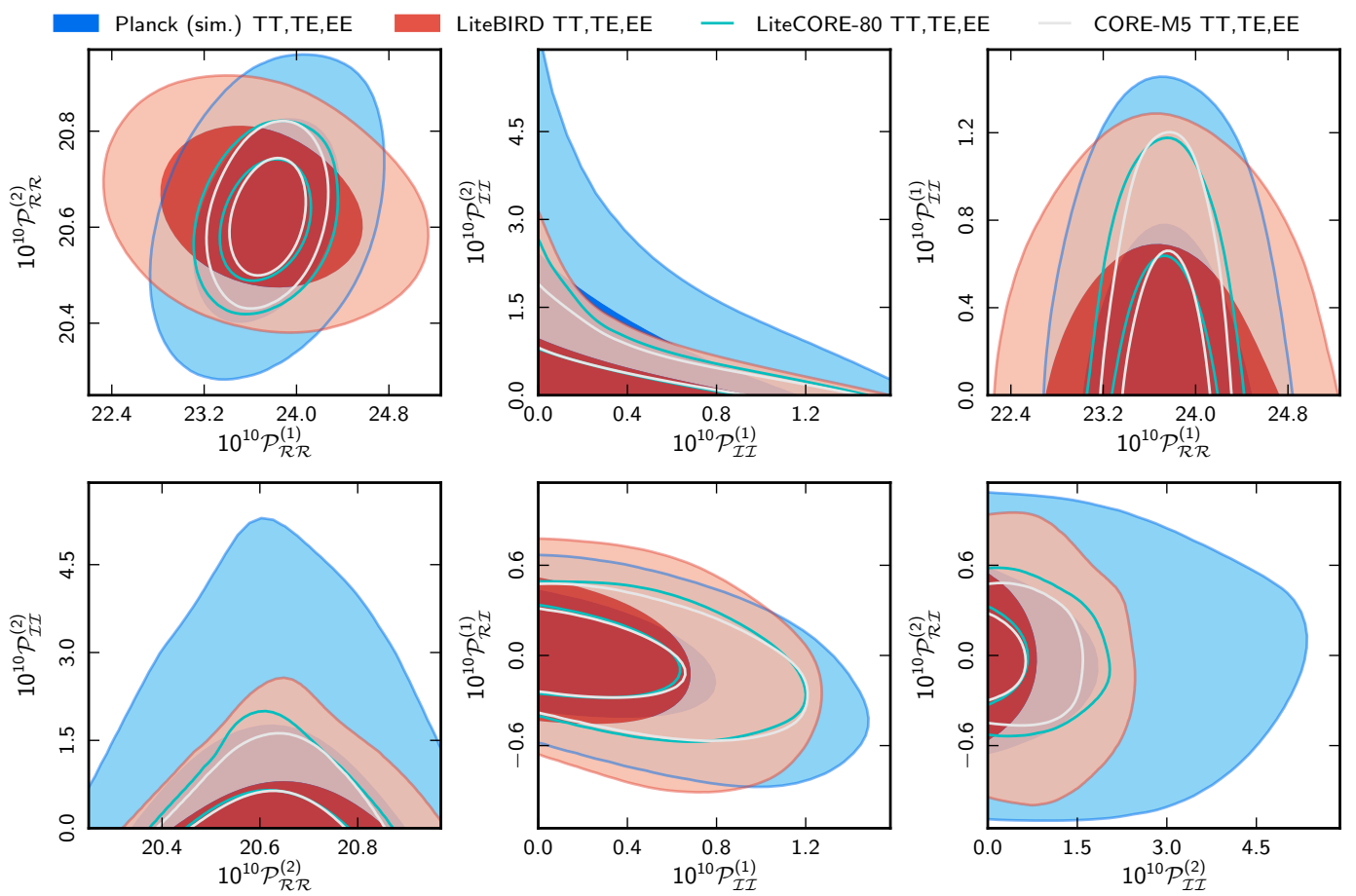

(b)
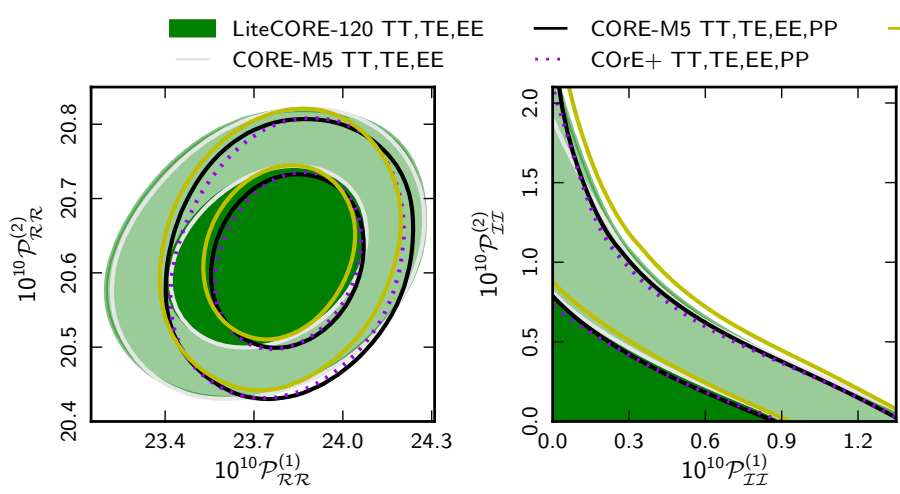

- Ideal TT,TE,EE Imax $=3000$
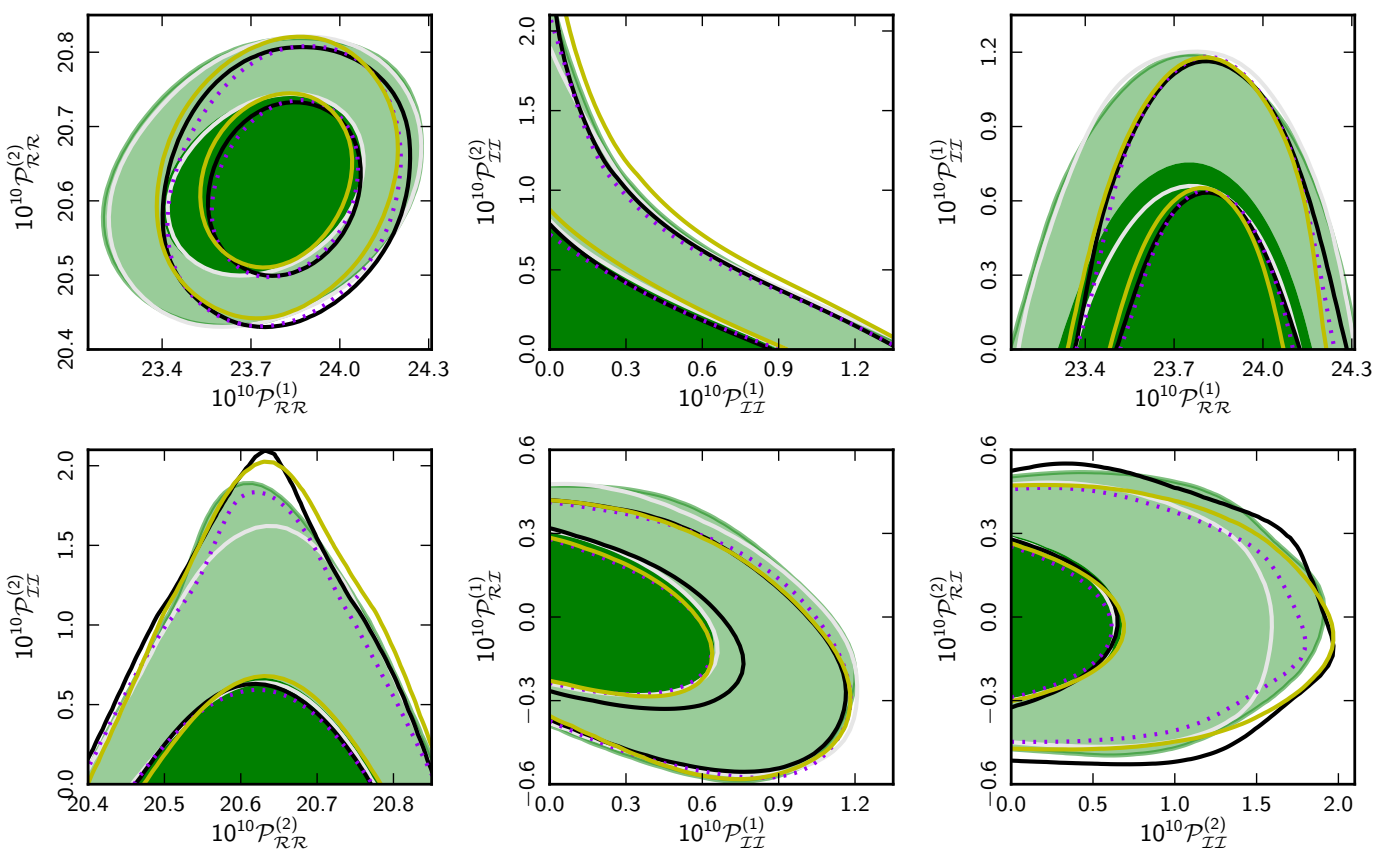

Figure 25: Constraints on primordial curvature, isocurvature and correlation power at large scales and small scales, denoted by the superscripts (1) and (2), respectively, when the fiducial data are adiabatic and have $r=0$, and the fitted model has generally correlated primordial adiabatic and CDI modes. (a) Simulated Planck and LiteBIRD data lead to significantly weaker constraints than CORE-M5, but LiteCORE-80 only slightly weaker. (b) A zoomed version, now with LiteCORE-120, and showing COREM5 and COrE + with the lensing potential PP. For the isocurvature and correlation powers LiteCORE-120, CORE-M5 and COrE + are virtually indistinguishable and reach the cosmic variance limit (the Ideal case). $\mathrm{PP}$ improves the constraints on the curvature power. 


\begin{tabular}{|l|c|c|c|cc|}
\hline & $100 \beta_{\text {iso }}\left(k_{\text {low }}\right)$ & $100 \beta_{\text {iso }}\left(k_{\text {mid }}\right)$ & $100 \beta_{\text {iso }}\left(k_{\text {high }}\right)$ & $100 \cos \Delta$ \\
\hline Planck $($ sim.) TT,TE,EE & 4.70 & 8.29 & 15.31 & -1.78 & {$[-20.81 ; 19.44]$} \\
LiteBIRD TT,TE,EE & 4.07 & 4.48 & 7.87 & -0.91 & {$[-24.63 ; 25.01]$} \\
(LiteBIRD TT,TE,EE,BB; $\left.r_{0.05}=10^{-3}\right)$ & 4.01 & 5.18 & 9.89 & -5.17 & {$[-23.04 ; 13.45]$} \\
LiteCORE-80 TT,TE,EE & 3.82 & 3.84 & 6.64 & -0.24 & {$[-15.76 ; 17.50]$} \\
LiteCORE-120 TT,TE,EE & 3.79 & 3.76 & 6.25 & -0.80 & {$[-15.25 ; 15.31]$} \\
CORE-M5 TT,TE,EE & 3.91 & 3.57 & 5.67 & -0.80 & {$[-15.51 ; 14.80]$} \\
CORE-M5 TT,TE,EE,PP & 3.73 & 3.63 & 6.40 & -1.26 & {$[-15.05 ; 14.79]$} \\
(CORE-M5 TT,TE,EE,BB,PP; $\left.r_{0.05}=10^{-3}\right)$ & 3.77 & 4.38 & 7.83 & -3.09 & {$[-16.21 ; 10.50]$} \\
COrE+ TT,TE,EE,PP & 3.75 & 3.54 & 6.02 & -1.56 & {$[-15.43 ; 13.56]$} \\
Ideal TT,TE,EE & 3.79 & 3.79 & 6.49 & -1.46 & {$[-15.05 ; 13.51]$} \\
\hline
\end{tabular}

Table 12: $95 \%$ CL upper bound on the primordial isocurvature fraction $\beta_{\text {iso }}$ at three different scales (from large scales to small scales), and the mean posterior value of the primordial correlation fraction and the 95\% CL interval of its one-dimensional marginalized posterior. The fitted model has a general correlation between the adiabatic and CDI modes, while the fiducial data assume pure adiabatic $\Lambda$ CDM with $r=0$, except on the lines with $r_{0.05}=10^{-3}$ (to be discussed in Section 7.4).

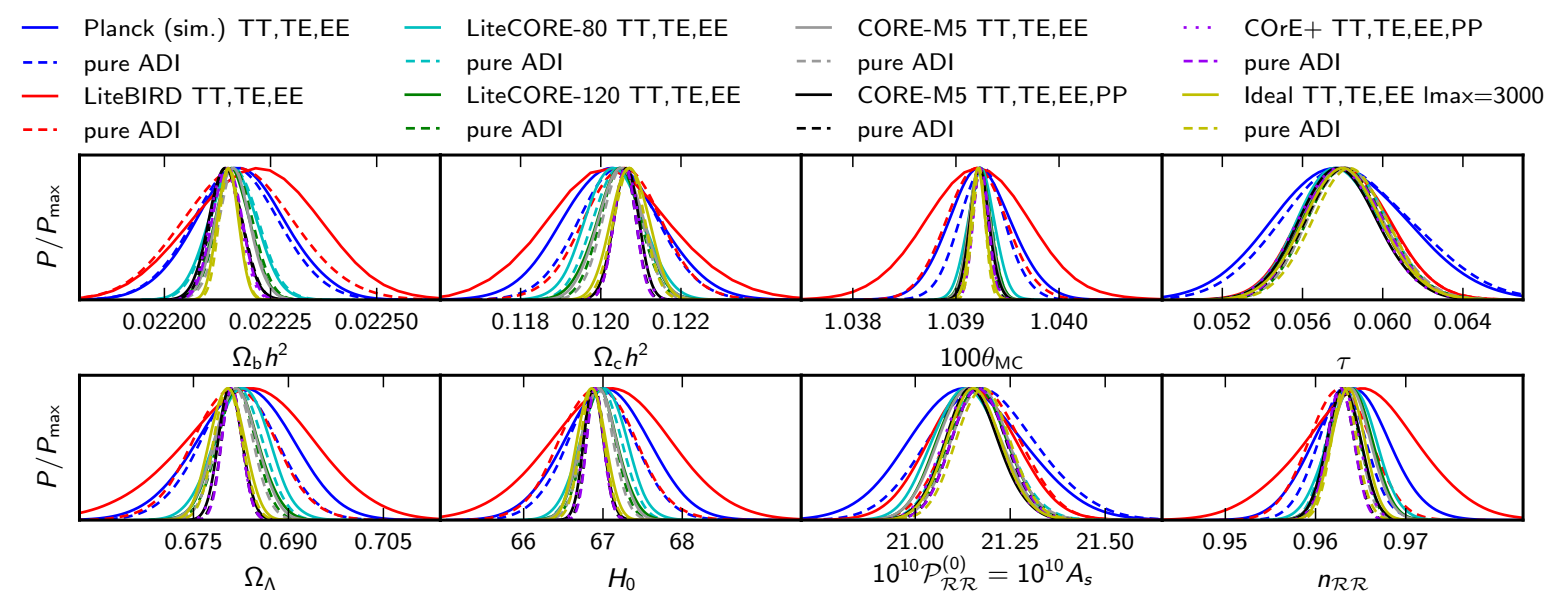

Figure 26: Parameters that exist also in the standard adiabatic model - comparison of their determination when assuming pure adiabatic model (dashed lines) or when assuming a generally correlated mixture of the adiabatic and CDI mode (solid lines). The fiducial data here are pure adiabatic with $r=0$.

except for LiteBIRD are near to the cosmic variance limit. The last panel of Table 12 shows the primordial correlation fraction. Interestingly, LiteBIRD constrains $\cos (\Delta)$ less well than Planck, while the other configurations reach the cosmic variance limit. CORE-M5 tightens the 95\% CL interval by a factor of 1.3 compared to Planck and by a factor of 1.7 compared to LiteBIRD.

Finally, in Fig. 26 and Table 13, we report selected standard cosmological parameters, which will be determined much better than by Planck (in general with $\sigma$ two to four times smaller), virtually at the cosmic variance limit. In particular, $H_{0}$ and $\Omega_{\mathrm{c}} h^{2}$ will be determined with excellent (almost ideal) accuracy no matter whether or not isocurvature is allowed in the theoretical model. There is a well-known degeneracy (different parameter combinations produce almost the same observable angular power spectrum) between the isocurvature parameters and the standard parameters (mainly $\theta_{\mathrm{MC}}$ and $\Omega_{\mathrm{b}} h^{2}$, but this also is reflected in other parameters). With the WMAP accuracy, these degeneracies cause large absolute shifts and significant broadening of the posterior of standard parameters when isocurvature is allowed [275, 280, 284, 285], but with the Planck data 


\begin{tabular}{|c|c|c|c|c|c|c|c|c|c|c|c|c|}
\hline & \multicolumn{2}{|c|}{$100 \Omega_{\mathrm{b}} h^{2}$} & \multicolumn{2}{|c|}{$100 \Omega_{\mathrm{c}} h^{2}$} & \multicolumn{2}{|c|}{$10000 \theta_{\mathrm{MC}}$} & \multicolumn{2}{|c|}{$100 \Omega_{\Lambda}$} & \multicolumn{2}{|c|}{$H_{0}$} & \multicolumn{2}{|c|}{$100 n_{\mathcal{R} \mathcal{R}}$} \\
\hline & mean & $\sigma$ & mean & $\sigma$ & mean & $\sigma$ & mean & $\sigma$ & hean & $\sigma$ & mean & $\sigma$ \\
\hline Fiducial data (a & 2.214 & & 12.06 & & 103.922 & & 68.10 & & 66.89 & & 96.25 & \\
\hline Planc & 2.218 & 0.011 & 12.03 & 0.12 & 103.924 & 0.031 & 68.30 & 0.76 & 67.05 & 0.53 & 96.39 & 0.41 \\
\hline & 2.217 & 0.010 & 12.05 & 0.10 & 103.926 & 0.023 & 68.20 & 0.62 & 66.98 & 0.44 & 96.29 & 0.27 \\
\hline LiteB & 2.221 & 0.014 & 12.02 & 0.16 & 103.921 & 0.051 & 68.34 & 1.04 & 67.09 & 0.76 & 96.49 & 0.64 \\
\hline & 2.217 & 0.013 & 12.05 & 0.10 & 103.920 & 0.029 & 68.14 & 0.63 & 66.94 & 0.47 & 96.27 & 34 \\
\hline (Lite & 2.215 & 0.010 & 12.08 & 0.06 & 103.905 & 0.040 & 67.91 & 0.41 & 66.76 & 0.31 & 96.24 & 0.42 \\
\hline & 2.213 & 0.010 & 12.07 & 0.05 & 103.921 & 28 & 68.03 & 0.32 & 66.84 & 0.25 & 96.23 & 0.30 \\
\hline Lite & 2.216 & 006 & 12.03 & 0.07 & 103.925 & 13 & 68.26 & 0.42 & 67.01 & 0.28 & 96.40 & 0.26 \\
\hline & 2.217 & 0.006 & 12.04 & 0.06 & 103.924 & 0.010 & 68.23 & 0.36 & 66.99 & 0.25 & 96.31 & 0.19 \\
\hline Lite & 2.216 & 4 & 12.05 & 0.06 & 103.923 & & 68.19 & 0 . & 6.96 & 0. & 96.40 & 0 . \\
\hline & 2.217 & 0.004 & 12.05 & 0.05 & 103.923 & 0.008 & 68.18 & 0.30 & 66.96 & 0.20 & 96.32 & 0.17 \\
\hline $\mathrm{COR}$ & 2.216 & 0.004 & 12.05 & 0.06 & 103.923 & & 8.17 & 0.33 & 66.95 & 0.22 & 96.39 & 0.22 \\
\hline pur & 2.217 & 0.004 & 12.05 & 0.05 & 103.923 & 0.008 & 68.15 & 0.29 & 66.94 & 0.19 & 96.32 & 0.17 \\
\hline CORI & 2.214 & 0.004 & 12.06 & 0.03 & 103.923 & & 68.09 & 0.19 & 66.89 & 0.13 & 96.32 & 0.19 \\
\hline pure & 2.215 & 0.004 & 12.06 & 0.03 & 103.924 & 0.008 & 68.11 & 0.16 & 66.90 & 0.11 & 96.28 & 0.15 \\
\hline $\mathrm{E}, \mathrm{BB}, \mathrm{PP}: \mathrm{CDI})$ & 2.214 & 0.004 & 12.07 & 0.03 & 103.920 & 0.009 & 68.06 & 0.19 & 66.86 & 0.13 & 96.27 & 0.18 \\
\hline (pur & 2.214 & 0.004 & 12.06 & 0.03 & 103.922 & 0.008 & 68.11 & 0.16 & 66.90 & 0.11 & 96.25 & 0.14 \\
\hline E,EE,PP: CDI & 2.214 & 0.003 & 12.06 & 0.03 & 103.923 & & 68.08 & 0.18 & 66.88 & 0.12 & 96.31 & 0.17 \\
\hline pure & 2.215 & 0.003 & 12.06 & 0.03 & 103.924 & 0.007 & 68.11 & 0.16 & 66.91 & 0.11 & 96.28 & 0.14 \\
\hline Ideal 7 & 2.215 & 0. & 12.07 & 0.05 & 103.922 & & 68.03 & 0.26 & 66.85 & 0.17 & 96.36 & 0.18 \\
\hline & 2.215 & 02 & 12.07 & 0 & 103.922 & & 68.05 & 0 . & 66.86 & 0.15 & 96.32 & 0 . \\
\hline
\end{tabular}

Table 13: Selected parameters that exist also in the standard adiabatic model - comparison of their recovered posterior mean value and posterior standard deviation when fitting the pure adiabatic model (pure ADI) or when fitting the general CDI model. The fiducial data here are pure adiabatic with $r=0$. (Note that even when fitting the pure adiabatic model, the last digits may slightly differ from the values given in the other Sections, since here our primary primordial perturbation parameters that have a uniform prior are $\mathcal{P}_{\mathcal{R} \mathcal{R}}^{(1)}$ and $\mathcal{P}_{\mathcal{R} \mathcal{R}}^{(2)}$, not $\ln \left[10^{10} A_{\mathrm{S}}\right]=\ln \left[10^{10} \mathcal{P}_{\mathcal{R} \mathcal{R}}\left(k_{\text {mid }}\right)\right]$ and $n_{\mathrm{S}}=n_{\mathcal{R} \mathcal{R}}$ like in the other Sections. Furthermore, here we do not utilize BB, except on the lines where the experiment name is in parenthesis where the fiducial adiabatic model has $r_{0.05}=10^{-3}$, to be discussed in Section 7.4.)

these effects almost disappear [5]. However CORE-M5 as well as both LiteCOREs and COrE + would further decrease the uncertainties caused by the degeneracy and make the determination of the standard parameters even more robust against the assumptions made concerning the initial conditions. (In Table 13, for each experiment we quote first the results when fitting the general correlated three-parameter isocurvature CDI model and then the results when fitting the pure adiabatic model.) Table 13 indicates only marginal broadening of the $1 \sigma$ interval with CDI and insignificant shifts compared to the input fiducial data, as indicated on the first line of the Table.

However with Planck and LiteBIRD some broadening and shifts (compared to the pure adiabatic fit, pure ADI/dashed lines) are visible in Fig. 26 and confirmed in the Table. For example, with LiteBIRD the $1 \sigma$ posterior interval of the adiabatic scalar spectral index $n_{\mathcal{R} \mathcal{R}}$ broadens by a factor of 1.9 when isocurvature is allowed, and the mean value of $n_{\mathcal{R} \mathcal{R}}$ shifts by $+0.25 \%$ compared to the input fiducial value, or by $+0.7 \sigma_{\mathrm{ADI}}$. With CORE-M5 TT, TE, EE, and PP, the corresponding numbers are very small: broadening by a factor of 1.3 (note that this is compared to the already small $\sigma_{\mathrm{ADI}}=0.0015$ of CORE-M 5 ) and a shift by $+0.07 \%$ or $+0.4 \sigma_{\mathrm{ADI}}$. 

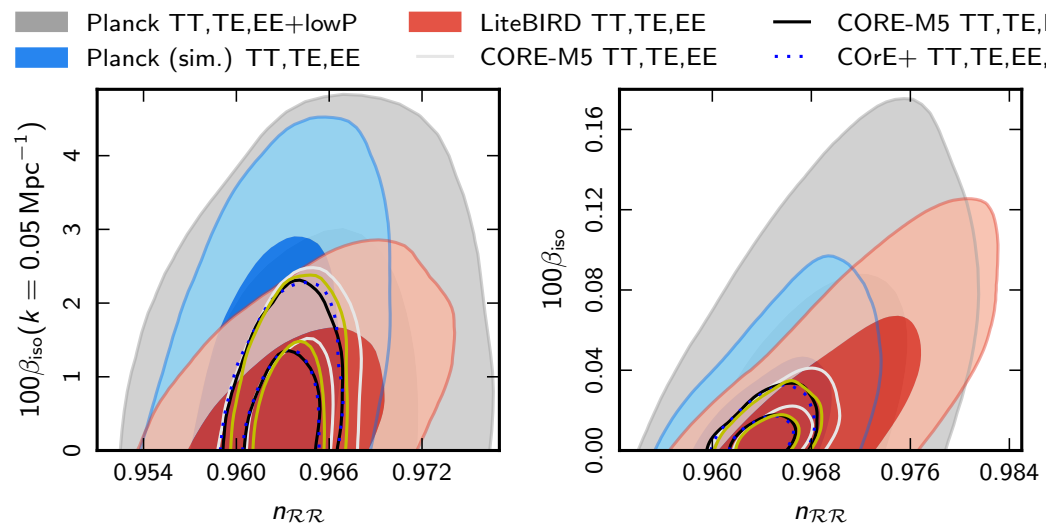

- Ideal TT,TE,EE Imax $=3000$

Figure 27: One-parameter isocurvature extensions to the adiabatic $\Lambda$ CDM model. Left: an "Axion" model (uncorrelated adiabatic and isocurvature modes, with $n_{\mathcal{I I}}=1, \cos \Delta=0$ ). Middle: "Curvaton I" (fully correlated adiabatic and isocurvature modes, with $n_{\mathcal{I} \mathcal{I}}=n_{\mathcal{R} \mathcal{R}}, \cos \Delta=+1$ ). Right: "Curvaton II", (fully anti-correlated adiabatic and isocurvature modes, with $n_{\mathcal{I I}}=n_{\mathcal{R} \mathcal{R}}, \cos \Delta=-1$ ). The simulated Planck and LiteBIRD datasets contain only TT,TE,EE, but the simulated CORE-M5 is presented both with and without the lensing potential PP (but not with the BB nor delensing information). The real Planck data contain also a noisy B-mode at low- $\ell$, consistent with zero. CORE-M5 has capability for an unprecedented constraining power in the curvaton models.

\begin{tabular}{|c|c|c|c|c|c|c|c|c|c|c|c|}
\hline & \multicolumn{3}{|c|}{ Axion (NC) } & \multicolumn{3}{|c|}{ Curvaton I (FC) } & \multicolumn{3}{|c|}{ Curvaton II (AC) } & \multicolumn{2}{|c|}{ Pure ADI } \\
\hline & $100 \beta_{\text {iso }}$ & $n_{\mathcal{R} \mathcal{R}}$ & $\sigma\left(n_{\mathcal{R} \mathcal{R}}\right)$ & $100 \beta_{\text {iso }}$ & $n_{\mathcal{R} \mathcal{R}}$ & $\sigma\left(n_{\mathcal{R} \mathcal{R}}\right)$ & $100 \beta_{\text {iso }}$ & $n_{\mathcal{R} \mathcal{R}}$ & $\sigma\left(n_{\mathcal{R} \mathcal{R}}\right)$ & $n_{\mathcal{R} \mathcal{R}}$ & $\sigma\left(n_{\mathcal{R} \mathcal{R}}\right)$ \\
\hline Planc & 3.842 & 0.9653 & 0.0044 & 0.129 & 0.9693 & 0.0049 & 0.082 & 0.9600 & 0.0048 & 0.9641 & 0.0044 \\
\hline Plan & 3.628 & 0.9628 & 0.0 & 072 & 0. & 0.0 & 072 & 0.9572 & 0.0034 & .9629 & 0.0027 \\
\hline Lite & .192 & 0.9647 & 0.0038 & 096 & 0 & 0.0050 & .072 & 0.9555 & 8 & 9627 & 0.0034 \\
\hline $\mathrm{CO}$ & 51 & 0.9 & 0.0018 & 031 & 56 & 19 & 0.022 & $\$ 12$ & 18 & .9632 & 0.0017 \\
\hline $\mathrm{CO}$ & 1.792 & 0.9633 & & 026 & 0.9645 & 0.0 & 0.021 & 0.9612 & & .9628 & 0.0015 \\
\hline $\mathrm{COr}$ & 1.823 & 0.9632 & 0.0015 & 0.025 & 0.9644 & 0.0015 & 0.021 & 0.9613 & 0.0015 & 0.9628 & 0.0014 \\
\hline Ideal TT,TE,EE & 1.891 & 0.9637 & 0.0015 & 0.026 & 0.9649 & 0.0016 & 0.021 & 0.9617 & 0.0015 & 0.9632 & 0.0015 \\
\hline
\end{tabular}

Table 14: 95\% CL upper bounds on the primordial isocurvature fraction $\beta_{\text {iso }}$, the posterior mean value of the scalar spectral index $n_{\mathcal{R} \mathcal{R}}=n_{\mathcal{I I}}=n_{\mathcal{R} \mathcal{I}}$, and $1 \sigma$ uncertainty in its determination when fitting the one-parameter isocurvature extensions of the $\Lambda \mathrm{CDM}$ model (the first three cases) and when fitting the pure adiabatic model (the last case). The fiducial data are the pure adiabatic $\Lambda$ CDM model, with $n_{\mathcal{R} \mathcal{R}}=0.9625, r=0\left(\right.$ and $\left.\beta_{\text {iso }}=0\right)$.

\subsubsection{Special one parameter extensions to adiabatic $\Lambda \mathrm{CDM}$}

There are a number of interesting (one-parameter) isocurvature models simpler than the general model studied in the previous Subsection. Here we analyze the same three models as in [3, 5]. These are an "Axion" model (no correlation between ADI and CDI, and $n_{\mathcal{I I}}=1$ and $r=0$; see, e.g., ref. [286]), "Curvaton I" (100\% correlation between ADI and CDI, and $n_{\mathcal{I I}}=n_{\mathcal{R} \mathcal{R}}$, and "Curvaton II" $\left(-100 \%\right.$ correlation between ADI and CDI, and $\left.n_{\mathcal{I I}}=n_{\mathcal{R} \mathcal{R}}\right)$. These curvaton models have $\cos \Delta= \pm 1$ and do not have a primordial tensor contribution since the tensor contribution is associated to the curvature perturbation at the horizon exit during inflation, which in these models is negligible. The primordial curvature perturbation is generated later from the conversion of the isocurvature perturbation into curvature perturbations [132-134], hence the full (anti)-correlation between them (see also [287]). Indeed, as discussed in [5, 212-214, 275], in 
slow-roll models if the tensor-to-scalar power ratio at the end of inflation was $\tilde{r}$, at the primordial epoch after inflation and reheating the ratio would become $r=\left(1-\cos ^{2} \Delta\right) \tilde{r}-$ or in terms of the slow-roll parameter $\epsilon, r=16 \epsilon \sin ^{2} \Delta$ to the leading order. Thus $r$ will be zero if $|\cos \Delta|=1$.

Constraints on these three models are presented in Fig. 27 and Table 14: "Axion" (no correlation, NC) in the left panel, "Curvaton I" in the middle panel (full correlation, FC), and "Curvaton II" in the right panel (anti-correlation, AC). In addition to the simulated Planck, LiteBIRD, CORE-M5, and COrE + data, we reproduce the constraint from the real Planck TT, TE, $\mathrm{EE}+$ lowP data as reported in Ref. [5].

For the axion model, the real and simulated Planck data constrain the primordial isocurvature fraction equally well. However the simulated Planck data constrain the primordial scalar spectral index $n_{\mathcal{R} \mathcal{R}}\left(=n_{\mathcal{I I}}\right)$ more tightly than the 2015 data, since the simulated data constrain the optical depth $\tau$ much better, at an accuracy similar to the subsequent determination using the Planck HFI data $[73,178]$. The 95\% upper bound on the isocurvature fraction drops from the Planck level of $4 \%$ to $2.2 \%$ with LiteBIRD and to $1.8 \%$ with CORE-M5 or COrE + .

The constraints on $\beta_{\text {iso }}$ are much tighter for the curvaton models, since the value of $\beta_{\text {iso }}$ directly controls the amplitude of the correlation power spectrum, which now is the geometric average of the (small) isocurvature power and the (larger) curvature perturbation power. Hence the correlation has a much larger effect on the CMB angular power spectrum than the isocurvature itself, which is the only contribution in the uncorrelated axion model. For curvaton models LiteBIRD performs equally well or worse than Planck, but CORE-M5 gives unprecedented constraining power. The upper bound on $\beta_{\text {iso }}$ drops from the Planck one part per thousand by almost an order of magnitude, to $0.26(\mathrm{FC})$ or 0.21 ( $\mathrm{AC})$ per thousand.

Figure 27 shows a degeneracy between the spectral index and isocurvature fraction in the curvaton models. Consequently, marginalizing over $\beta_{\text {iso }}$ biases the one-dimensional marginalized posterior of $n_{\mathcal{R} R}$ toward larger values in the Curvaton I (FC), and toward smaller values in the Curvaton II (AC) case. This is evident in Table 14, where for example with the simulated Planck data the posterior mean value of $n_{\mathcal{R} \mathcal{R}}$ is 0.9655 with $\mathrm{FC}(0.9 \sigma$ above the input fiducial value of $0.9625)$ or 0.9572 with $\mathrm{AC}\left(1.6 \sigma\right.$ below the fiducial value). As CORE-M5 constrains $\beta_{\text {iso }}$ much better, the absolute shift in the mean value of $n_{\mathcal{R} \mathcal{R}}$ is significantly reduced (see for example the line in bold in the Table), but since the width $(\sigma)$ of the posterior also shrinks, the shift naturally stays at the same $1 \sigma$ level as with Planck.

\subsection{Fiducial data with a fully (anti)-correlated CDI contribution}

Isocurvature perturbations do not produce B modes except through lensing exactly as in the adiabatic model without tensors. Therefore BB and delensing information would not significantly help for the studies described in the previous Subsections. However, in this Subsection we utilize $\mathrm{BB}$, since we fit to our fiducial data a model where $\tilde{r}$ is a free parameter, while $n_{\mathrm{t}}$ is fixed by the inflationary consistency condition to $n_{\mathrm{t}}=-2 \epsilon=-r /\left(8\left(1-\cos ^{2} \Delta\right)\right)=-\tilde{r} / 8$. We create two fiducial datasets, one assuming "Curvaton I" (i.e., $100 \%$ correlated ADI and CDI) with $\beta_{\text {iso }}=0.1 \%$, and another assuming "Curvaton II" with the same isocurvature fraction (but setting 100\% anticorrelation between ADI and CDI). As explained in the previous Subsection, these fiducial data do not have any primordial tensor contribution. However we fit to these data our full three parameter generally correlated isocurvature model (CDI) and also allow for a nonzero $\tilde{r}$. In a nutshell, our 

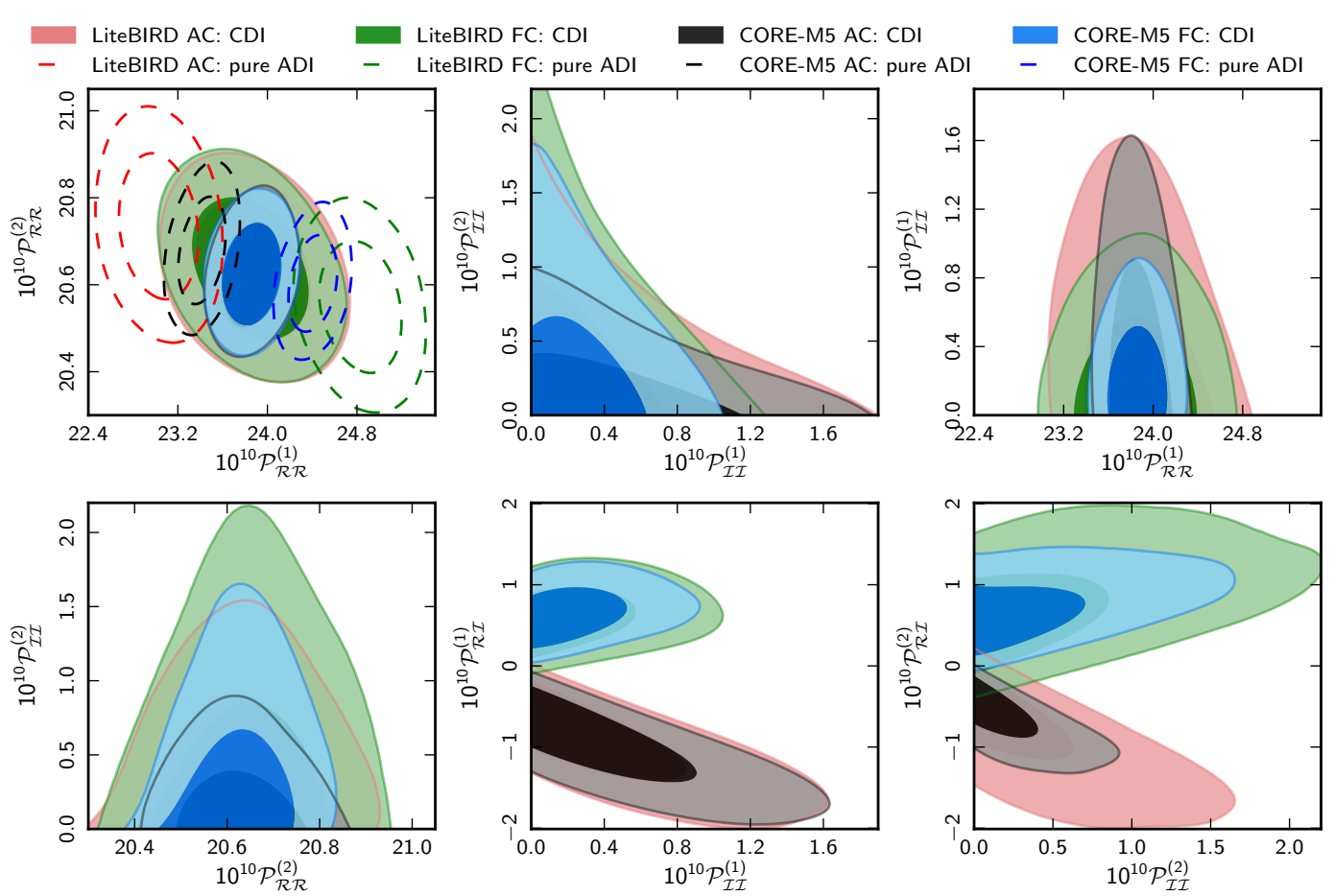

Figure 28: Primordial powers when the fiducial data are produced from a curvaton model and have a $0.1 \%$ anti-correlated (AC) or $0.1 \%$ fully correlated (FC) CDI contribution, but no tensor contribution. The fitted model is the generally correlated model with three isocurvature parameters and $\tilde{r}_{0.05}$ (CDI, shaded colors) or the pure adiabatic model with $r_{0.05}$ (pure ADI, dashed contours). LiteBIRD uses TT,TE,EE,BB, whereas CORE-M5 uses TT,TE,EE,BB,PP and delensing information. (The fiducial values used are $10^{10} \mathcal{P}_{\mathcal{R} \mathcal{R}}^{(1)}=23.90,10^{10} \mathcal{P}_{\mathcal{R} \mathcal{R}}^{(2)}=20.64,10^{10} \mathcal{P}_{\mathcal{I I}}^{(1)}=0.0239,10^{10} \mathcal{P}_{\mathcal{I} \mathcal{I}}^{(2)}=0.0206,10^{10} \mathcal{P}_{\mathcal{R} \mathcal{I}}^{(1)}= \pm 0.7560$, $10^{10} \mathcal{P}_{\mathcal{R} \mathcal{I}}^{(2)}= \pm 0.6528$.) For the dramatically biased posterior of $\mathcal{P}_{\mathcal{R} \mathcal{R}}$ in the first panel in the case when we fit the wrong (pure ADI, dashed lines) model, see the main text and footnote 12.

fiducial data has only one extra parameter compared to the tensorless adiabatic $\Lambda$ CDM model, but we try to fit to a theory with four extra parameters. For comparison, we show the dramatic effects encountered in determining the standard cosmological parameters if we try to force fit the pure adiabatic model (ADI) to these data having a seemingly small one part per thousand primordial isocurvature fraction.

Figure 28 demonstrates that both LiteBIRD and CORE-M5 easily recover the adiabatic and correlation input parameters of these models (though the input isocurvature power is too small to be detected). In particular, the bottom middle and right panels show that the nonzero correlation is detected at about the $2 \sigma$ level. However Fig. 29 and Table 15 reveal that the isocurvature spectral index (input fiducial value $n_{\mathcal{I I}}=n_{\mathcal{R} \mathcal{R}}=0.9625$ ) is poorly constrained, CORE-M5 being about $15 \%$ better than LiteBIRD. The difference in the capability to set an upper bound on $r$ is also evident from the first panels of Fig. 29 and Table 15. When fitting a wrong model (i.e., the pure adiabatic model) to these data, the constraints on $r$ change insignificantly. In addition, we show in Table 15 the results when fitting the full CDI model without a tensor contribution: the constraints on $\mathcal{P}_{\mathcal{I} \mathcal{I}}^{(1)}, \mathcal{P}_{\mathcal{R} \mathcal{I}}^{(1)}, n_{\mathcal{I} \mathcal{I}}$, and $\mathcal{P}_{\mathcal{R} \mathcal{R}}^{(1)}$ stay virtually unchanged. Consequently, allowing for a nonzero $r$ does not bias the results nor weaken the constraints. Moreover, this test proves 


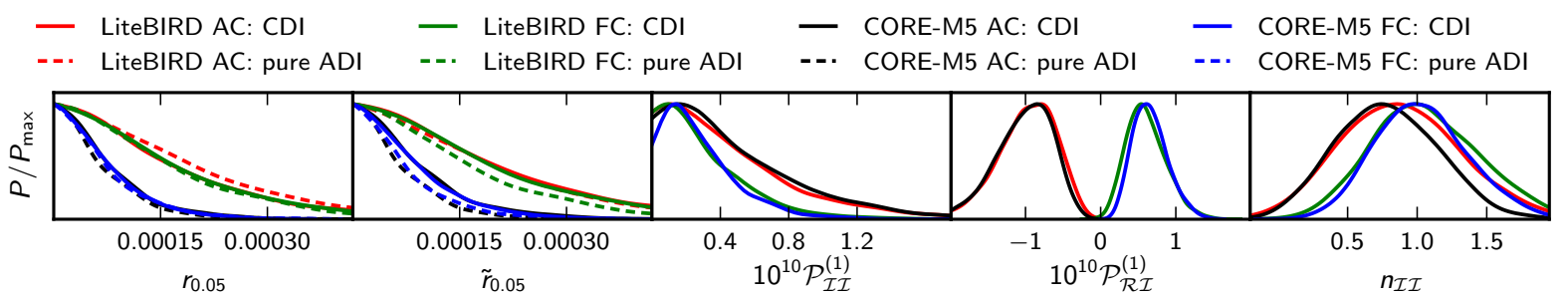

Figure 29: The primordial tensor-to-scalar ratio $r_{0.05}$, the horizon exit tensor-to-scalar ratio $\tilde{r}_{0.05}$, isocurvature and correlation power at large scales, and isocurvature spectral index, when the fiducial data have a $0.1 \%$ anti-correlated (AC) or fully correlated (FC) CDI contribution and $r=0$. The fitted model is the generally correlated model with three isocurvature parameters and $\tilde{r}_{0.05}$ (CDI, solid lines) or the pure adiabatic $\Lambda \mathrm{CDM}$ model with $r_{0.05}$ (pure ADI, dashed lines).

that the asymmetry in the determination of $\mathcal{P}_{\mathcal{R I}}$ between the $\mathrm{AC}$ and FC cases is not caused by a (hypothetical) degeneracy between $r$ and the correlation contribution. Even without $r$, the posterior on $10^{10} \mathcal{P}_{\mathcal{R} \mathcal{I}}^{(1)}$ in the $\mathrm{AC}$ case has a long tail toward much more negative values than the fiducial value -0.76 , leading to a biased mean value -1.0 of the posterior with both LiteBIRD and CORE-M5. In the FC case the posterior on $\mathcal{P}_{\mathcal{R} \mathcal{I}}^{(1)}$ is much more symmetric and centers near to the fiducial value +0.76 . This asymmetry between $\mathrm{AC}$ and $\mathrm{FC}$ cases can be understood as follows. When the full CDI fit has a negative correlation, the positive contribution from the isocurvature itself can partially cancel the correlation contribution, thus explaining the more negative correlation to fit the data. However when the full CDI fit has a positive correlation, it adds to the positive contribution from isocurvature itself, and hence we obtain tighter constraints in the case of positive correlation for both $\left|\mathcal{P}_{\mathcal{R} \mathcal{I}}^{(1)}\right|$ and $\mathcal{P}_{\mathcal{I} \mathcal{I}}^{(1)}$. This degeneracy is confirmed in Table 15 (and in the bottom middle panel of Fig. 28).

To summarize, fitting the three isocurvature degrees of freedom (CDI) or ignoring them (pure ADI) does not significantly interfere with the upper bound on $r$. Neither does allowing for a nonzero $r$ interfere with the determination of the isocurvature parameters in these models with a one part per thousand fully (anti)-correlated isocurvature contribution. Furthermore, apart from the determination of the isocurvature spectral index, there is no significant difference in the (isocurvature) detection power between LiteBIRD and CORE-M5. However $r$ is constrained about 2.2 times better by CORE-M5 in these models.

In Fig. 30 and Table 16 we study a different question: What happens to the determination of the standard cosmological parameters if we (unaware of the one part per thousand AC/FC isocurvature contribution in the data) fit the pure adiabatic model (pure ADI) to these data? When fitting the full CDI model, we recover the input fiducial model with very high accuracy. On the contrary, force fitting a wrong model (pure ADI) leads to a huge bias in the standard parameters, as large as $3 \sigma$. However, in particular with CORE-M5, the shifts are small in terms of percentage of the input fiducial values. With this measure, the largest shifts with CORE-M5 are for $\Omega_{\mathrm{c}} h^{2}, \Omega_{\Lambda}$, and $n_{\mathcal{R} \mathcal{R}}$ whose values can be corrupted by $0.6 \%$. In case of LiteBIRD, the value of $\Omega_{\Lambda}$ can be misestimated by fitting the pure adiabatic model by as much as $1.3 \%$.

Most of the shifts seen in Fig. 30 and Table 16 can be traced back to the determination of the sound horizon angle $\theta_{\mathrm{MC}}$. As first pointed out in [282] and later in [285, 288] (when fitting isocurvature models to the WMAP data), the positive correlation effectively shifts the acoustic 


\begin{tabular}{|c|c|c|c|c|c|c|c|c|c|}
\hline & $\begin{array}{l}10^{4} r_{0.05} \\
95 \% \text { CL }\end{array}$ & $\begin{array}{l}10^{4} \tilde{r}_{0.05} \\
95 \% \text { CL }\end{array}$ & $\begin{array}{r}10^{10} \mathcal{P}_{\mathcal{I} \mathcal{I}}^{(1)} \\
95 \% \mathrm{CL}\end{array}$ & mean & $\begin{array}{l}0^{10} \mathcal{P}_{\mathcal{R} \mathcal{I}}^{(1)} \\
68 \% \text { CL }\end{array}$ & mean & $\begin{array}{l}100 n_{\mathcal{I I}} \\
68 \% \text { CL }\end{array}$ & mean & $\begin{array}{l}0^{10} \mathcal{P}_{\mathcal{R} \mathcal{R}}^{(1)} \\
68 \% \text { CL }\end{array}$ \\
\hline Fiducial data & 0 & 0 & 0.024 & \pm 0.76 & & 96.25 & & 23.90 & \\
\hline LiteBIRD AC: CDI & $<4.11$ & $<4.92$ & $<1.275$ & -0.99 & {$[-1.29 ;-0.54]$} & 88.42 & {$[42.26 ; 128.28]$} & 23.88 & {$[23.54 ; 24.21]$} \\
\hline$r=0 \mathrm{CDI}$ & 0 & 0 & $<1.263$ & -0.99 & {$[-1.29 ;-0.53]$} & 87.35 & {$[39.81 ; 125.19]$} & 23.86 & {$[23.54 ; 24.19]$} \\
\hline pure ADI & $<4.43$ & $<4.43$ & & & & & & 23.03 & {$[22.81 ; 23.25]$} \\
\hline LiteBIRD FC: CDI & $<3.94$ & $<4.54$ & $<0.818$ & 0.61 & {$[0.31 ; 0.82]$} & 107.04 & {$[63.71 ; 141.74]$} & 23.85 & {$[23.52 ; 24.18]$} \\
\hline$r=0 \mathrm{CDI}$ & & 0 & $<0.885$ & 0.58 & {$[0.29 ; 0.79]$} & 107.63 & {$[63.58 ; 142.59]$} & 23.88 & {$[23.52 ; 24.21]$} \\
\hline pure ADI & $<3.67$ & $<3.67$ & & & & & & 24.83 & {$[24.60 ; 25.07]$} \\
\hline CORE-M5 AC: CDI & $<1.84$ & $<2.19$ & $<1.288$ & -1.02 & {$[-1.32 ;-0.59]$} & 78.45 & {$[39$} & 23.86 & {$[23.69 ; 24.02]$} \\
\hline$r=0 \mathrm{CDI}$ & 0 & 0 & $<1.306$ & -1.04 & {$[-1.34 ;-0.61]$} & 76.76 & {$[37.09 ; 109.63]$} & 23.85 & {$[23.68 ; 24.01]$} \\
\hline pure ADI & $<1.68$ & $<1.68$ & & & & & & 23.42 & {$[23.29 ; 23.55$} \\
\hline CORE-M5 FC: CDI & $<1.74$ & $<2.02$ & $<0.717$ & 0.66 & {$[0.40 ; 0.85]$} & 103.21 & {$[67.26 ; 133.28]$} & 23.86 & {$[23.69 ; 24.03]$} \\
\hline$r=0 \mathrm{CDI}$ & & 0 & $<0.737$ & 0.66 & {$[0.40 ; 0.85]$} & 102.15 & {$[66.18 ; 132.75]$} & 23.87 & {$[23.70 ; 24.04]$} \\
\hline pure ADI & $<1.82$ & $<1.82$ & & & & & & 24.41 & {$[24.27 ; 24.54$} \\
\hline
\end{tabular}

Table 15: The same information in numbers as in Fig. 29, with the curvature perturbation amplitude at large scales added as a last column, and we also compare the full CDI with and without $r$.

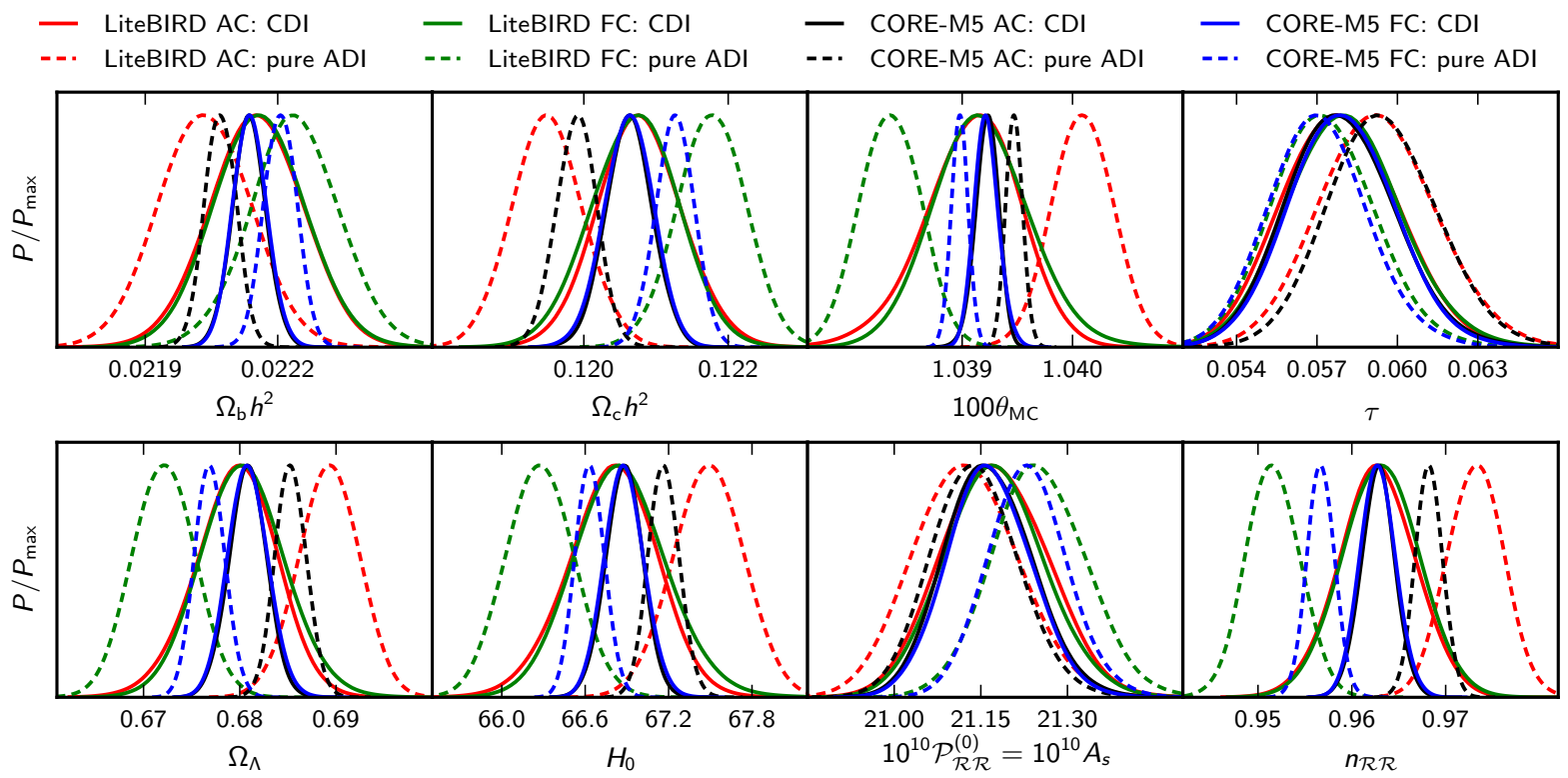

Figure 30: Determination of the standard parameters when the fiducial data have a $0.1 \%$ anti-correlated (AC) or fully correlated (FC) CDI contribution and zero $r$. The solid lines (CDI) represent the fit of the generally correlated three isocurvature parameter model plus $\tilde{r}_{0.05}$ to these one isocurvature parameter models. The input fiducial data are recovered extremely well in this case. However, despite of the smallness of the isocurvature contribution, fitting a "wrong" model, i.e., the pure adiabatic model (pure ADI, dashed lines), to these data leads to a large bias (error) in the determination of the standard parameters.

peaks toward the right, leading to a smaller estimated $\theta_{\mathrm{MC}}$, and the negative correlation leads to a larger estimated $\theta_{\mathrm{MC}}$ if the other parameters are kept fixed. By reversing the argument, we realize that having an $\mathrm{AC}$ component in the data but fitting the pure adiabatic model gives us a too large $\theta_{\mathrm{MC}}$, while with the $\mathrm{FC}$ data fitting the pure adiabatic model gives us a too small $\theta_{\mathrm{MC}}$. These changes are then reflected in $\Omega_{\mathrm{m}} h^{2}$, and further in $H_{0}, \Omega_{\Lambda}$, and the other parameters. The adiabatic spectral index $n_{\mathcal{R} \mathcal{R}}$ is further affected by the ratio of the large scale to small scale 


\begin{tabular}{|c|c|c|c|c|c|c|c|c|c|c|c|c|c|c|c|c|c|c|}
\hline & $\begin{array}{r}10 \\
\text { mean }\end{array}$ & $\begin{array}{l}0 \Omega_{\mathrm{b}} h \\
\Delta(\sigma)\end{array}$ & & $\begin{array}{r}10 \\
\text { mean }\end{array}$ & $\begin{array}{l}0 \Omega_{\mathrm{c}} h \\
\Delta(\sigma)\end{array}$ & $\Delta(\%)$ & $\begin{array}{c}100 \\
\text { mean }\end{array}$ & $\begin{array}{l}00 \theta_{\mathrm{M}} \\
\Delta(\sigma)\end{array}$ & & $\begin{array}{r}1 \\
\text { mean }\end{array}$ & $\begin{array}{l}00 \Omega_{1} \\
\Delta(\sigma)\end{array}$ & $\Delta(\%)$ & mean & $\begin{array}{c}H_{0} \\
\Delta(\sigma)\end{array}$ & $\Delta(\%)$ & $\begin{array}{r}10 \\
\text { mean }\end{array}$ & $\begin{array}{l}0 n_{\mathcal{R}}^{\mathcal{T}} \\
\Delta(\sigma)\end{array}$ & \\
\hline Fiducia & 2.21 & & & 12.06 & & & 103.92 & & & 68.10 & & & 66.89 & & & 96.25 & & \\
\hline LB A & 2.22 & 0.1 & 0.1 & 12.08 & 0.3 & 0.1 & 103.91 & -0.3 & -0.0 & 67.96 & -0.3 & -0.2 & 66.80 & -0.3 & -0.1 & 96.27 & 0.1 & 0.0 \\
\hline & .20 & -1.0 & -0.5 & 11.95 & -1.9 & -0.9 & 4.01 & 2.0 & 0.1 & 68.92 & 2.0 & 1.2 & 19 & 1.9 & 0.9 & 97.32 & 6 & 1. \\
\hline LB FC: CDI & 2.22 & 0.2 & 0.1 & 12.07 & 0.2 & 0.1 & 103.92 & -0.1 & -0.0 & 68.03 & -0.2 & -0.1 & 66.85 & -0.1 & -0.1 & 96.31 & 0.1 & 0.1 \\
\hline & 2.22 & 0.9 & 0.4 & 12.18 & 1.9 & 1.0 & 103.83 & -2.0 & -0.1 & 67.21 & -2.0 & -1.3 & 66.27 & -1.8 & -0.9 & 95.16 & -2.6 & -1.1 \\
\hline CORE-M5 AC: CDI & 2.21 & -0.1 & -0.0 & 12.06 & 0.1 & 0.0 & 103.92 & 0.0 & 0.0 & 68.08 & -0.1 & -0.0 & 66.88 & -0.1 & -0.0 & 96.28 & 0.2 & 0. \\
\hline & 2.21 & -1.8 & -0.3 & 11.99 & -2.2 & -0.6 & 103.95 & 2.4 & 0.0 & 68.53 & 2.2 & 0.6 & 67.17 & 2.1 & 0.4 & 96.82 & 3.1 & 0.6 \\
\hline CORE- & 2.21 & -0.1 & -0.0 & 12.06 & 0.1 & 0.0 & 103.92 & 0.0 & 0.0 & 68.08 & -0.1 & -0.0 & 66.88 & -0.1 & -0.0 & 96.28 & 0.1 & 0. \\
\hline pure ADI & 2.22 & 1.7 & 0.3 & 12.13 & 1.9 & 0.5 & 103.90 & -2.2 & -0.0 & 67.69 & -2.0 & -0.6 & 66.63 & -1.8 & -0.4 & 95.67 & -3.0 & -0.6 \\
\hline
\end{tabular}

Table 16: Determination of the standard parameters when the fiducial data have a $0.1 \%$ anti-correlated (AC) or fully correlated (FC) CDI contribution and zero $r . \Delta$ indicates the shift (bias) with respect to the input fiducial values in units of the standard deviation $(\sigma)$ of the posterior of the full three-parameter CDI model plus $\tilde{r}_{0.05}$ or in percentage (\%). Fitting a wrong model, the pure adiabatic model (pure ADI), leads to seemingly large biases (as large as $|\Delta|=3 \sigma$ ) in terms of $\sigma$, but all shifts are below $|\Delta|=1.3 \%$.

(temperature) angular power. Since the $\mathrm{AC} / \mathrm{FC}$ contribution with $n_{\mathcal{I} \mathcal{I}}=n_{\mathcal{R} \mathcal{R}}$ only affects the low multipoles (large scales), the ratio is reduced (increased) in the $\mathrm{AC}$ (FC) case, leading to a too large (small) $n_{\mathcal{R} \mathcal{R}}$ if the pure adiabatic model is fitted to these data. ${ }^{12}$

In the case of these simulated $\mathrm{AC} / \mathrm{FC}$ data, it is naturally easy to tell that the pure adiabatic model is the wrong model, since with LiteBIRD the best fit $\chi^{2}$ of pure ADI is about 30 points worse and with CORE-M5 about 60 points worse than the one of CDI. However, with real data the question is more subtle. For example, is 12060 an acceptable best fit $\chi^{2}$ with 12000 "data points", or should one seriously look for another model beyond the pure adiabatic $\Lambda$ CDM model? Indeed, the first panel of Fig. 28 helps answer this question, at least with respect to the isocurvature extensions. Large shifts in the "ADI contours" when a generally correlated CDI mode is allowed constitute a clear hint that the CDI model may be the correct one. Conversely, if the contours do not shift, there is very little or no room for isocurvature.

\subsection{Adiabatic fiducial data plus a tensor contribution}

Our final test of isocurvature is with pure adiabatic fiducial data plus a tensor contribution with $r_{0.05}=10^{-3}$ and $n_{\mathrm{t}}=-r_{0.05} / 8$. We fit to these data the full three-parameter generally correlated isocurvature model, and a free $\tilde{r}$ (with $n_{\mathrm{t}}=-\tilde{r} / 8$ ). LiteBIRD with TT, TE, EE, BB and COREM5 with TT, TE, EE, BB, PP, and delensing constrains the isocurvature power equally well to near zero (see Fig. 31). However CORE-M5 constrains the correlation power both at large scales

\footnotetext{
${ }^{12}$ The large-scale Sachs-Wolfe effect is such that $\delta T / T \approx-(1 / 5)\left(\mathcal{R}+2 f_{c} \mathcal{S}\right)$, where $f_{c}=\Omega_{\mathrm{c}} /\left(\Omega_{\mathrm{c}}+\Omega_{\mathrm{b}}\right)$ and $\mathcal{P}_{\mathcal{R} \mathcal{R}} \propto\left\langle|\mathcal{R}|^{2}\right\rangle, \mathcal{P}_{\mathcal{I I}} \propto\left\langle|\mathcal{S}|^{2}\right\rangle$, and $\mathcal{P}_{\mathcal{R I}} \propto\left\langle\mathcal{R}^{*} \mathcal{S}\right\rangle$ at the primordial time [280]. If $b^{2} \equiv \mathcal{P}_{\mathcal{I I}} / \mathcal{P}_{\mathcal{R} \mathcal{R}}$, then $C_{\ell}^{T T} \propto$ $\left\langle(\Delta T / T)^{2}\right\rangle \propto\left(1 \pm 2 \times 2|b| f_{c}+4 b^{2} f_{c}^{2}\right) \mathcal{P}_{\mathcal{R} \mathcal{R}}$. Now we have $f_{c}=0.8451$ and $b \approx 0.0316$, which leads to $C_{\ell}^{T T} \propto 0.90 \mathcal{P}_{\mathcal{R} \mathcal{R}}$ (AC, the minus sign) and $1.11 \mathcal{P}_{\mathcal{R} \mathcal{R}}$ (FC, the plus sign). Thus, our one part per thousand primordial isocurvature contribution translates to a $\pm 10 \%$ observational non-adiabatic contribution at large scales. (In addition to the ordinary Sachs-Wolfe effect, the large-scale angular power is affected by the integrated Sachs-Wolfe effect. A precise numerical examination of the temperature angular power spectrum of our fiducial model shows that the maximum non-adiabatic contribution, coming mainly from correlation, is actually $\pm 8 \%$ at multipole $\ell=5$, and after $\ell \approx 65$ the non-adiabatic contribution falls below $1 \%$, being $\pm 0.6 \%$ to the first acoustic peak at $\ell \approx 220$.) This together with the change in $\theta_{\mathrm{MC}}$ explains the dramatic shifts of $\mathcal{P}_{\mathcal{R} \mathcal{R}}^{(1)}$ in the upper left panel of Fig. 28 when incorrectly fitting the pure ADI model.
} 

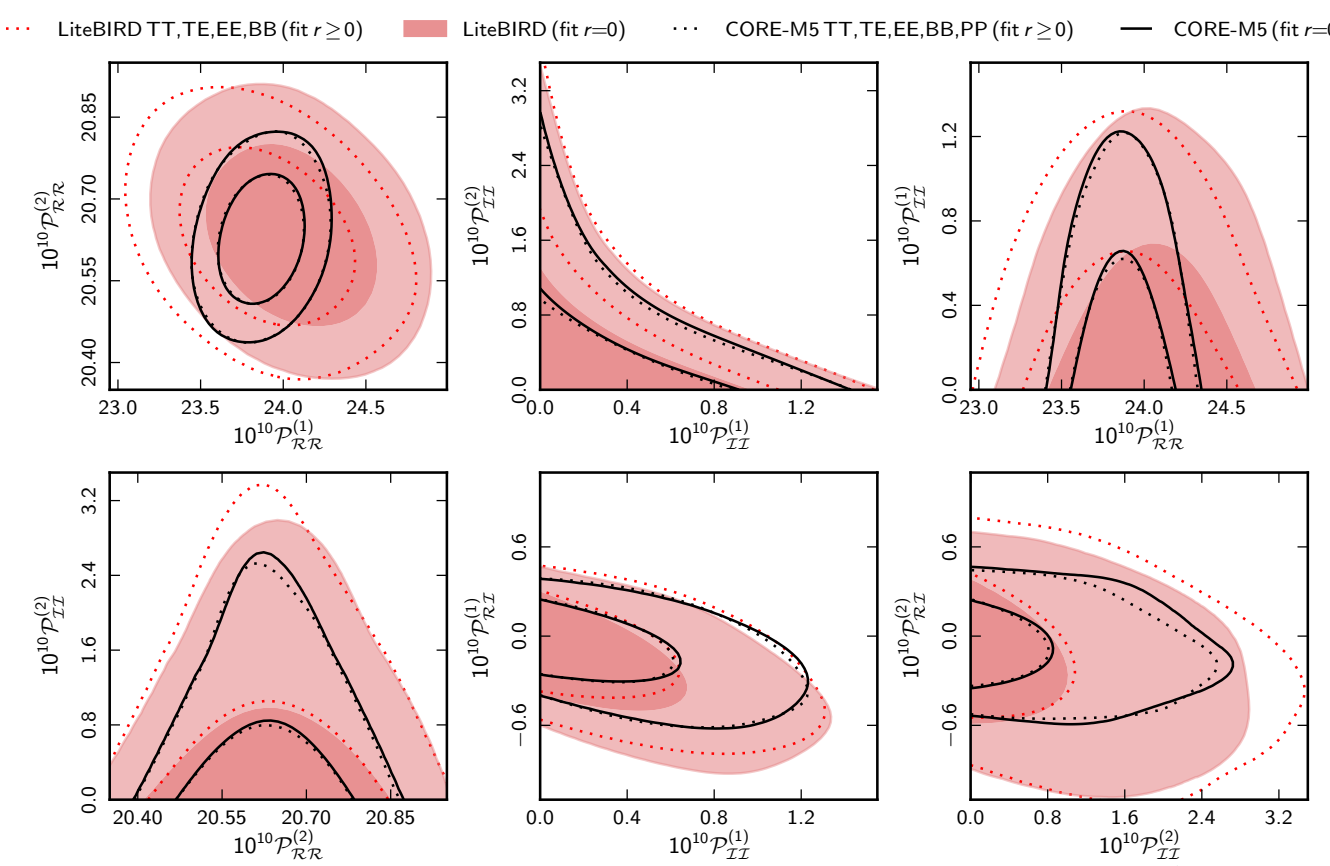

Figure 31: Primordial powers when the fiducial data are adiabatic with $r_{0.05}=10^{-3}$. The fitted model is the generally correlated CDI model with three isocurvature parameters and a free $\tilde{r}_{0.05}$ or a fixed $r=0$. CORE-M5 utilizes delensing and its results with a free $\tilde{r}_{0.05}$ or with $r=0$ are indistinguishable.

and small scales (last two panels of the Figure) about two times closer to zero than LiteBIRD. Figure 31 shows also the results of fitting a wrong model (i.e, the $r=0$ model) to these data that in reality have $r_{0.05}=10^{-3}$. Overall, this does not significantly affect the determination of isocurvature parameters. In particular, CORE-M5 does not misidentify the tensor contribution as an additional isocurvature or correlation contribution, but with LiteBIRD the correlation power becomes slightly more biased toward negative values.

The input value of $r_{0.05}=10^{-3}$ is recovered with CORE-M5 much better than with LiteBIRD, see also Section 4. When fitting the CDI model, a slightly larger $r$ is favored than by fitting the pure adiabatic model. This can be understood by looking at the bottom middle panel of Fig. 31. The negative correlation power at large scales, which is more pronounced than the positive correlation power, leaves more room for a positive tensor contribution to the temperature angular power spectrum and hence allows for a larger $r$ to fit the data. Likewise, to make more room for the positive TT contribution from $r, \beta_{\text {iso }}$ at large scales becomes smaller (with LiteBIRD) and $\cos \Delta$ more negative, as reported in Table 12 in Section 7.2.1.

\subsection{Summary}

Depending on the details of the model and the measure used, CORE-M5 determines the nature (the pure adiabatic mode or possibly a correlated mixture of adiabatic and isocurvature modes) of primordial perturbations 2-5 times better than Planck. LiteBIRD, on the other hand, cannot improve much on Planck because of its coarse angular resolution. Downgrading CORE-M5 to LiteCORE-120 does not affect the constraining power what comes to isocurvature. Downgrading to LiteCORE-80 shows insignificant weakening of constraints. On the other hand, upgrading to 
COrE + does not improve the results, since already CORE-M5 is virtually cosmic variance limited what comes to probing the isocurvature perturbations. In cases where both the primordial tensor contribution and an isocurvature mode are present, CORE-M5 distinguishes between these better than LiteBIRD.

\section{Primordial non-Gaussianity}

This Section investigates the implications of the different configurations proposed for the new CORE satellite for studying primordial non-Gaussianity (NG). In most cases, we also compare the forecasts to the current state-of-the-art Planck results [52] as well as to forecasts for the proposed LiteBIRD satellite and a hypothetical ideal noiseless experiment.

Results are presented as predicted $1 \sigma$ error bars of the so-called $f_{\mathrm{NL}}$ and $g_{\mathrm{NL}}$ parameters, measuring the amplitudes of various theoretical bispectrum and trispectrum templates. The bispectrum and connected trispectrum are the Fourier or spherical harmonic transform of the three-point and four-point correlation function, respectively, and would be zero in the purely Gaussian case. Bispectrum (trispectrum) templates arising from different inflationary scenarios display a specific functional dependence on triangular (quadrilateral) configurations in Fourier space. Such a dependence defines the bispectrum shape. Unless otherwise stated, all bispectrum and trispectrum shapes analyzed here are considered independent.

So far the best constraints on primordial NG have been obtained from direct measurements of the angular bispectrum and trispectrum of CMB temperature and polarization fluctuations. However, new promising techniques have recently been proposed. Such techniques rely on measurements of either CMB spectral distortion anisotropies - tiny departures of the CMB energy spectrum from that of a blackbody across the sky - or the cosmic infrared background (CIB), and they can lead, for specific shapes and using future surveys, to better constraints than those achievable through standard bispectrum and trispectrum measurements. We will analyze bispectrum and trispectrum constraints in Section 8.1, and consider these new approaches in Section 8.2. We conclude in Section 8.3.

\subsection{CMB temperature and polarization bispectrum and trispectrum}

\subsubsection{Standard bispectrum shapes}

We start by analyzing the so-called standard bispectrum shapes: local, equilateral, and orthogonal (sometimes collectively called LEO), as well as the foreground lensing-ISW bispectrum due to correlations between the integrated Sachs-Wolfe (ISW) effect and gravitational lensing. More information about these shapes can be found in $[52,289]$ and references therein. Our $f_{\mathrm{NL}}$ forecasts are obtained via a standard bispectrum Fisher matrix analysis. The signal-to-noise ratio is given by (see e.g., [290, 291] for details)

$$
\left(\frac{S}{N}\right)^{2}=\frac{1}{6} \sum_{\ell_{1} \ell_{2} \ell_{3}} \frac{B_{\ell_{1} \ell_{2} \ell_{3}}^{2}}{C_{\ell_{1}} C_{\ell_{2}} C_{\ell_{3}}}
$$

where $B_{\ell_{1} \ell_{2} \ell_{3}}$ is the CMB angular averaged bispectrum, computed for a given shape assuming $f_{\mathrm{NL}}=1$. Both the bispectrum and power spectrum include beam and noise, computed by 


\begin{tabular}{l|ccccccc} 
& LiteCORE & LiteCORE & CORE & COrE + & Planck & LiteBIRD & ideal \\
& 80 & 120 & M5 & & 2015 & & 3000 \\
\hline T local & 4.5 & 3.7 & $\mathbf{3 . 6}$ & 3.4 & $(5.7)$ & 9.4 & 2.7 \\
T equilat & 65 & 59 & $\mathbf{5 8}$ & 56 & $(70)$ & 92 & 46 \\
T orthog & 31 & 27 & $\mathbf{2 6}$ & 25 & $(33)$ & 58 & 20 \\
T lens-isw & 0.15 & 0.11 & $\mathbf{0 . 1 0}$ & 0.09 & $(0.28)$ & 0.44 & 0.07 \\
\hline E local & 5.4 & 4.5 & $\mathbf{4 . 2}$ & 3.9 & $(32)$ & 11 & 2.4 \\
E equilat & 51 & 46 & $\mathbf{4 5}$ & 43 & $(141)$ & 76 & 31 \\
E orthog & 24 & 21 & $\mathbf{2 0}$ & 19 & $(72)$ & 42 & 13 \\
E lens-isw & 0.37 & 0.29 & $\mathbf{0 . 2 7}$ & 0.24 & & 1.1 & 0.14 \\
\hline T+E local & 2.7 & 2.2 & $\mathbf{2 . 1}$ & 1.9 & $(5.0)$ & 5.6 & 1.4 \\
T+E equilat & 25 & 22 & $\mathbf{2 1}$ & 20 & $(43)$ & 40 & 15 \\
T+E orthog & 12 & 10.0 & $\mathbf{9 . 6}$ & 9.1 & $(21)$ & 23 & 6.7 \\
T+E lens-isw & 0.062 & 0.048 & $\mathbf{0 . 0 4 5}$ & 0.041 & & 0.18 & 0.027
\end{tabular}

Table 17: Forecasts for the $1 \sigma f_{\mathrm{NL}}$ error bars for the standard primordial shapes as well as for the lensing-ISW shape for the indicated configurations. Results are given for $T$-only, $E$-only and full $T+E$. The results for Planck have been put in parentheses because they are not forecasts but real measured error bars. See the main text for further details.

coadding cosmological channels for the different configurations considered (LiteCORE, COrE+, CORE-M5, together with Planck and LiteBIRD for comparison). Details of these configurations have been presented in Section 3. The forecasts are shown in Table 17. We remind the reader that the CORE configurations assume $\ell_{\max }=3000$ (except the LiteCORE-80 configuration that has $\ell_{\max }=2400$ ), while the much lower resolution LiteBIRD has $\ell_{\max }=1350$. A sky masked to leave $70 \%$ available has been assumed for all results. For comparison we also present the error bars of the Planck 2015 release. We stress that these are real error bars, not forecasts. Moreover, as far as polarization is concerned they are not yet the ultimate Planck error bars, since the 2015 polarization analysis was restricted to $\ell_{\min }=40$, which has an impact in particular on the local shape. The Planck analysis used $\ell_{\max }=2500$ for temperature and 2000 for $E$-polarization. Finally we also show for comparison the forecasts for an ideal full-sky and noiseless experiment with $\ell_{\max }=3000$.

Compared to Planck we see that the CORE configurations provide just a modest improvement in temperature-only (as expected since Planck already is nearly cosmic variance limited in temperature), but a very significant improvement in polarization-only, for a final improvement in full $T+E$ of about a factor of 2 . There is very little difference between the different CORE configurations. Because of its lower resolution, LiteBIRD performs significantly worse than CORE, with final $T+E$ error bars comparable to the current ones from Planck and hence about a factor of 2 worse than CORE. We also see that CORE performs within $50 \%$ of the ultimate error bars of an ideal noiseless full-sky experiment. It is also very interesting to note that CORE should provide a more than $20 \sigma$ detection of the predicted $f_{\mathrm{NL}}=1$ lensing-ISW bispectrum, which was for the first time observed by Planck but at only $3 \sigma$ significance. At this level of detection, it might be interesting to start using the ISW-lensing signal to estimate cosmological parameters. 
A graphical comparison between forecasts for different experiments and configurations is shown in Fig. 32, where we consider the $E$-only and $T+E$ case. As a figure of merit, in order to summarize the overall improvement achievable with a CORE-like experiment for the three standard shapes, we also computed the overall constrained volume in the three-dimensional localequilateral-orthogonal bispectrum space (accounting for correlations between them and using the shape correlator to define a scalar product between bispectra). We find that going from Planck to CORE shrinks the volume of the allowed $f_{\mathrm{NL}}$ parameter region by a factor $\approx 20$, using $T+E$, with little difference between the LiteCORE and COrE + configurations. The improvement reaches a level of $\approx 200$ if we consider polarization data only. Besides the improvements in error bars, we stress that having EEE measurements at the same level of sensitivity as TTT is important because it allows a much tighter control of systematics and foreground contamination, via internal cross-validation of $T$-only and $E$-only results.

Even in the absence of a detection, tight bounds on $f_{\mathrm{NL}}$ parameters are of course very important to constrain parameters in different inflationary scenarios. As an example of this, in Fig. 33 we forecast constraints on the inflaton speed of sound in the effective field theory of single-field inflation, derived from our equilateral and orthogonal $f_{\mathrm{NL}}$ predictions. In this case (see [94] and also [144]) a lower bound $c_{\mathrm{s}}>0.045$ (95\% CL) can be achieved, improving by almost $50 \%$ the present Planck constraints.

\subsubsection{Isocurvature non-Gaussianity}

In addition to these standard shapes, it is also interesting to investigate other shapes. One class of shapes where one would expect a significant improvement compared to Planck is isocurvature non-Gaussianity. If there was more than one degree of freedom during inflation, it is possible for one or more isocurvature modes to have survived in addition to the standard adiabatic mode. Such an additional mode will not only potentially produce a signal in the power spectrum (see Section 7), but also in the bispectrum. It should be noted that some inflation-curvaton models (e.g. [292]) predict an even larger isocurvature than adiabatic bispectrum and at the same time a negligible isocurvature power spectrum.

As explained in [293, 294], in the case of a local bispectrum produced by two modes, one adiabatic and one isocurvature, there will be six different $f_{\mathrm{NL}}$ parameters: one purely adiabatic ( $a, a a$; which is the normal adiabatic local shape), one purely isocurvature $(i, i i)$ and four mixed $(a, a i ; a, i i ; i, a a ; i, a i)$. Assuming a cosmology with a standard particle content, the isocurvature mode can be cold dark matter (CDM) density isocurvature, neutrino density isocurvature, or neutrino velocity isocurvature. ${ }^{13}$ As explained in [293, 294], several of the isocurvature NG modes profit significantly from the addition of polarization data to the analysis, much more than for the adiabatic mode. Already for Planck the improvement due to including polarization was up to a factor of six.

The forecasts for the isocurvature NG modes are given in Tables 18, 19, and 20, for the CDM density, neutrino density, and neutrino velocity isocurvature modes, respectively. We present both the case where all six modes are considered independent and the more realistic case when they are

\footnotetext{
${ }^{13}$ There could also be a baryon density isocurvature mode, but its shape is identical to the CDM density isocurvature mode, just with a rescaled amplitude.
} 

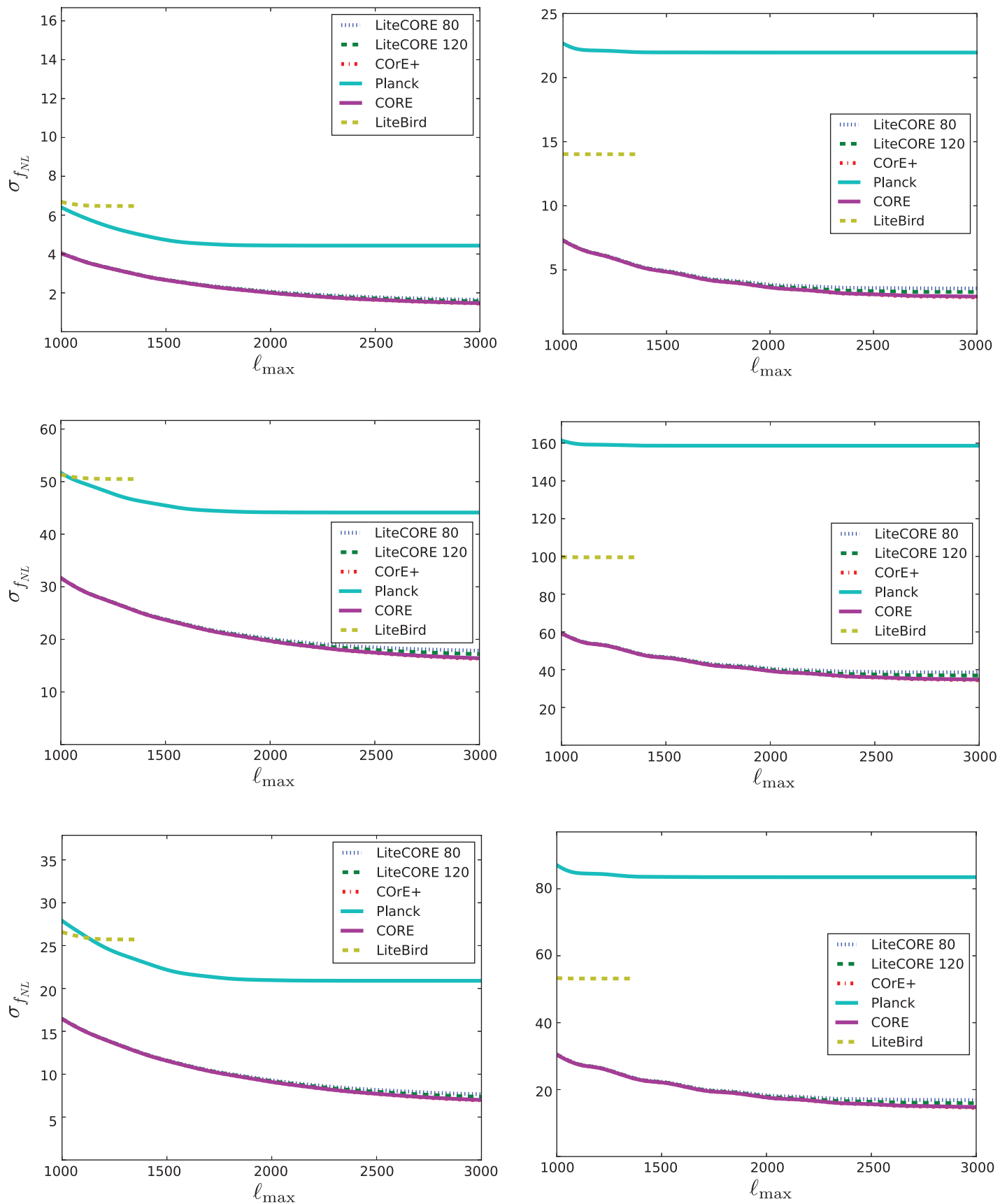

Figure 32: Expected local (first row), equilateral (second row), orthogonal (third row) $f_{\mathrm{NL}}$ error bars, obtained by combining temperature and polarization, on the left side, and using only polarization data, on the right side, for the different CORE configurations. The forecasts are compared to current constraints from Planck.

analyzed in a fully joint way. ${ }^{14}$ Like for the standard shapes we see that the difference between

\footnotetext{
${ }^{14}$ The independent $a, a a$ result in these Tables corresponds exactly with the local result in Table 17 . The small difference in the Planck column is due to a difference between estimators: the Planck errors cited in Table 17 are
} 


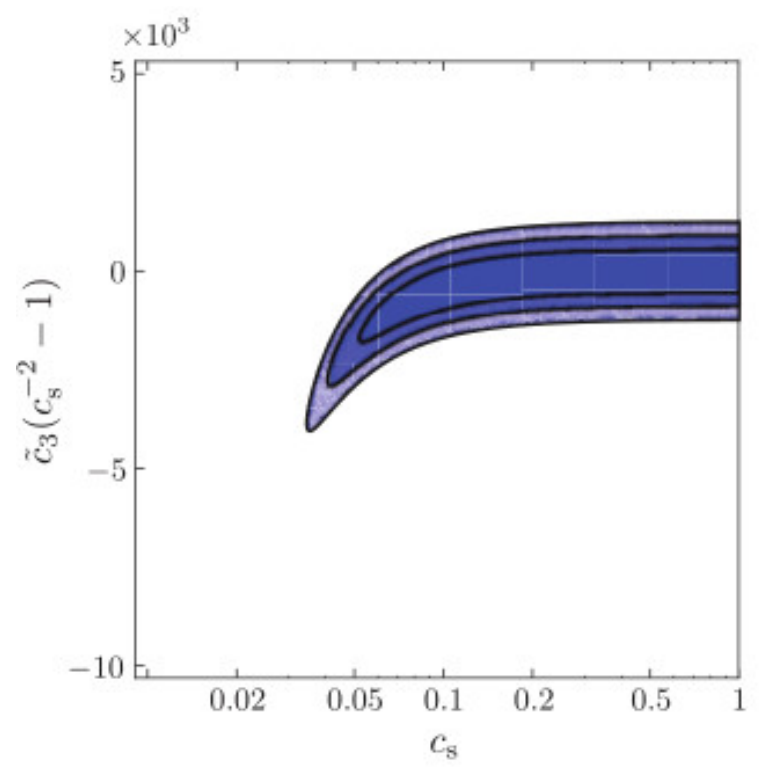

Figure 33: CORE-M5 forecasts of typical parameters of the effective field theory of inflation for general single-field models as obtained from the equilateral and orthogonal $f_{\mathrm{NL}}$ predictions $(68 \%, 95 \%$ and $99.7 \%$ confidence regions are shown). Vanishing central values for $f_{\mathrm{NL}}^{\text {equil }}$ and $f_{\mathrm{NL}}^{\text {ortho }}$ have been assumed. There are two "microscopic" non-Gaussianity parameters of interest. $c_{\mathrm{S}}$ is the sound speed of the inflaton field, with $\left(1-c_{\mathrm{s}}^{-2}\right)$ being the amplitude of the inflaton self-interaction $\pi(\nabla \pi)^{2}$, while $\tilde{c}_{3}$ is related to the amplitude of the inflaton self-interaction $(\dot{\pi})^{3}$.

the different CORE configurations is small. However, here the improvement compared to Planck in $T+E$ is much more impressive: for the joint analysis the improvement varies from a factor of 3 up to almost a factor of 10 for the neutrino velocity isocurvature mode. We also see the huge importance of polarization: for some shapes the error bars in the joint analysis decrease by a factor of almost 30 when going from $T$-only to $T+E$. Like for the standard shapes, we see that for all isocurvature modes CORE approaches to within $50 \%$ the ultimate error bars of an ideal noiseless and full-sky experiment.

\subsubsection{Spectral index of the bispectrum}

We also considered models with a mild running of the NG parameter $f_{\mathrm{NL}}$, parameterized by a spectral index $n_{\mathrm{NG}}$. Scale dependence of this type can arise, for example in single-field models with non-standard kinetic terms or in specific multi-field scenarios. The bispectrum in our forecast is written as a scale-independent part (typically, one of the standard local, equilateral, orthogonal shapes) multiplying a scale-dependent term, proportional to $\left(k_{1}+k_{2}+k_{3}\right)^{n_{\mathrm{NG}}}$, as in [295, 296]. Results in the $f_{\mathrm{NL}}-n_{\mathrm{NG}}$ plane from a full $T+E$ analysis are plotted in Fig. 34, while marginalized error bars are reported in Table 21. CORE allows an improvement of a factor of $>3$ when constraining the NG spectral index, with respect to Planck. We note that these models are not constrained yet using Planck data; constraints for local scale-dependent NG exist from WMAP

those computed by the KSW estimator, while the errors in the isocurvature Tables are those computed by the binned estimator. 


\begin{tabular}{l|ccccc|ccccc} 
& \multicolumn{5}{|c|}{ independent } & \multicolumn{7}{|c}{ joint ansis } & \multicolumn{7}{c}{ ansis } \\
& LC120 & C & C + & Planck & ideal & LC120 & C & C + & Planck & ideal \\
\hline T a,aa & 3.7 & $\mathbf{3 . 6}$ & 3.4 & $(5.4)$ & 2.7 & 9.7 & $\mathbf{9 . 4}$ & 8.8 & $(13)$ & 7.1 \\
T a,ai & 7.1 & $\mathbf{6 . 9}$ & 6.4 & $(10)$ & 5.2 & 19 & $\mathbf{1 8}$ & 17 & $(26)$ & 14 \\
T a,ii & 990 & $\mathbf{9 9 0}$ & 990 & $(910)$ & 830 & 7800 & $\mathbf{7 8 0 0}$ & 7800 & $(8200)$ & 6500 \\
T i,aa & 54 & $\mathbf{5 4}$ & 54 & $(50)$ & 45 & 120 & $\mathbf{1 2 0}$ & 120 & $(120)$ & 100 \\
T i,ai & 70 & $\mathbf{7 0}$ & 70 & $(66)$ & 58 & 950 & $\mathbf{9 5 0}$ & 940 & $(1000)$ & 790 \\
T i,ii & 290 & $\mathbf{2 9 0}$ & 290 & $(280)$ & 240 & 1900 & $\mathbf{1 8 0 0}$ & 1800 & $(2000)$ & 1500 \\
\hline E a,aa & 4.5 & $\mathbf{4 . 2}$ & 3.9 & $(34)$ & 2.4 & 7.9 & $\mathbf{7 . 3}$ & 6.7 & $(50)$ & 4.1 \\
E a,ai & 16 & $\mathbf{1 5}$ & 13 & $(200)$ & 8.2 & 27 & $\mathbf{2 6}$ & 23 & $(310)$ & 14 \\
E a,ii & 610 & $\mathbf{6 1 0}$ & 610 & $(4000)$ & 500 & 1100 & $\mathbf{1 1 0 0}$ & 1100 & $(6100)$ & 930 \\
E i,aa & 20 & $\mathbf{2 0}$ & 20 & $(87)$ & 17 & 39 & $\mathbf{3 8}$ & 38 & $(180)$ & 31 \\
E i,ai & 41 & $\mathbf{4 1}$ & 41 & $(250)$ & 34 & 130 & $\mathbf{1 3 0}$ & 130 & $(770)$ & 100 \\
E i,ii & 220 & $\mathbf{2 1 0}$ & 210 & $(2200)$ & 180 & 440 & $\mathbf{4 4 0}$ & 440 & $(5300)$ & 360 \\
\hline T+E a,aa & 2.2 & $\mathbf{2 . 1}$ & 1.9 & $(4.9)$ & 1.4 & 3.3 & $\mathbf{3 . 1}$ & 2.9 & $(10)$ & 2.0 \\
T+E a,ai & 5.3 & $\mathbf{5 . 1}$ & 4.7 & $(9.7)$ & 3.3 & 8.0 & $\mathbf{7 . 6}$ & 7.0 & $(20)$ & 4.9 \\
T+E a,ii & 200 & $\mathbf{2 0 0}$ & 200 & $(450)$ & 170 & 480 & $\mathbf{4 7 0}$ & 470 & $(1300)$ & 390 \\
T+E i,aa & 10 & $\mathbf{1 0}$ & 10 & $(26)$ & 8.6 & 19 & $\mathbf{1 9}$ & 19 & $(47)$ & 16 \\
T+E i,ai & 16 & $\mathbf{1 6}$ & 16 & $(38)$ & 13 & 54 & $\mathbf{5 4}$ & 53 & $(170)$ & 44 \\
T+E i,ii & 76 & $\mathbf{7 6}$ & 76 & $(170)$ & 63 & 140 & $\mathbf{1 4 0}$ & 140 & $(390)$ & 110
\end{tabular}

Table 18: Forecasts for the $1 \sigma$ local $f_{\mathrm{NL}}$ error bars with both an adiabatic and a CDM density isocurvature mode for the indicated configurations and showing both the independent and joint analysis. Results are given for $T$-only, $E$-only and full $T+E$. The results for Planck have been put in parentheses because they are not forecasts but real measured error bars. See the main text for further details.

data [297]. Therefore the Planck constraints in Table 21 also refer to a Fisher matrix analysis in this case.

\subsubsection{Oscillatory bispectra}

A strong scale-dependent running and sinusoidal oscillations in the CMB bispectrum can be produced in a variety of inflationary scenarios, generally characterized by temporary violations of the slow-roll conditions. These can arise in single-field inflation from the presence of sharp features in the inflaton potential or from changes in the inflaton sound speed. Large field models based on string theory, such as axion monodromy, can also produce this behavior, as well as multifield scenarios with sharp turns in field space. See for example [52] and references therein for a more detailed survey and explanations. It is interesting to note that in all the scenarios above, we not only have oscillatory bispectra, but also model-dependent oscillatory counterparts in the power spectrum and trispectrum. An observation of matching features in the power spectrum and bispectrum would of course enhance the statistical evidence in favor of specific models. Searches of this type have been conducted in [298, 299].

We consider here two specific primordial oscillatory shapes. One of them associated with sharp 


\begin{tabular}{l|ccccc|ccccc} 
& \multicolumn{5}{|c|}{ independent analysis } & \multicolumn{5}{c}{ joint analysis } \\
& LC120 & C & C + & Planck & ideal & LC120 & C & C + & Planck & ideal \\
\hline T a,aa & 3.7 & $\mathbf{3 . 6}$ & 3.4 & $(5.4)$ & 2.7 & 41 & $\mathbf{4 0}$ & 38 & $(52)$ & 31 \\
T a,ai & 10.4 & $\mathbf{1 0 . 1}$ & 9.5 & $(15)$ & 7.6 & 160 & $\mathbf{1 6 0}$ & 160 & $(210)$ & 130 \\
T a,ii & 190 & $\mathbf{1 9 0}$ & 170 & $(280)$ & 140 & 3700 & $\mathbf{3 6 0 0}$ & 3500 & $(4500)$ & 2900 \\
T i,aa & 6.1 & $\mathbf{6 . 0}$ & 5.6 & $(9.0)$ & 4.5 & 84 & $\mathbf{8 3}$ & 81 & $(99)$ & 66 \\
T i,ai & 15 & $\mathbf{1 5}$ & 14 & $(22)$ & 11 & 540 & $\mathbf{5 3 0}$ & 520 & $(630)$ & 430 \\
T i,ii & 170 & $\mathbf{1 7 0}$ & 160 & $(250)$ & 130 & 2200 & $\mathbf{2 1 0 0}$ & 2100 & $(2400)$ & 1700 \\
\hline E a,aa & 4.5 & $\mathbf{4 . 2}$ & 3.9 & $(34)$ & 2.4 & 22 & $\mathbf{2 1}$ & 20 & $(120)$ & 14 \\
E a,ai & 17 & $\mathbf{1 6}$ & 15 & $(140)$ & 8.9 & 107 & $\mathbf{1 0 2}$ & 96 & $(640)$ & 68 \\
E a,ii & 300 & $\mathbf{2 9 0}$ & 270 & $(2300)$ & 170 & 1300 & $\mathbf{1 2 0 0}$ & 1200 & $(6200)$ & 900 \\
E i,aa & 6.7 & $\mathbf{6 . 3}$ & 5.9 & $(42)$ & 3.7 & 35 & $\mathbf{3 4}$ & 33 & $(170)$ & 24 \\
E i,ai & 19 & $\mathbf{1 8}$ & 17 & $(130)$ & 11 & 180 & $\mathbf{1 7 0}$ & 170 & $(850)$ & 130 \\
E i,ii & 200 & $\mathbf{1 9 0}$ & 180 & $(1400)$ & 130 & 910 & $\mathbf{8 8 0}$ & 860 & $(5300)$ & 650 \\
\hline T+E a,aa & 2.2 & $\mathbf{2 . 1}$ & 1.9 & $(4.9)$ & 1.4 & 8.9 & $\mathbf{8 . 6}$ & 8.2 & $(27)$ & 6.1 \\
T+E a,ai & 7.0 & $\mathbf{6 . 6}$ & 6.2 & $(14)$ & 4.4 & 35 & $\mathbf{3 4}$ & 33 & $(94)$ & 24 \\
T+E a,ii & 120 & $\mathbf{1 2 0}$ & 110 & $(240)$ & 77 & 550 & $\mathbf{5 4 0}$ & 520 & $(1400)$ & 410 \\
T+E i,aa & 3.4 & $\mathbf{3 . 2}$ & 3.0 & $(7.7)$ & 2.2 & 15 & $\mathbf{1 5}$ & 14 & $(45)$ & 11 \\
T+E i,ai & 8.7 & $\mathbf{8 . 4}$ & 7.9 & $(19)$ & 5.7 & 70 & $\mathbf{6 9}$ & 67 & $(210)$ & 53 \\
T+E i,ii & 82 & $\mathbf{7 9}$ & 76 & $(180)$ & 57 & 300 & $\mathbf{2 9 0}$ & 290 & $(860)$ & 230 \\
& & & & & & & & & &
\end{tabular}

Table 19: Same as Table 18, but for the neutrino density isocurvature mode.
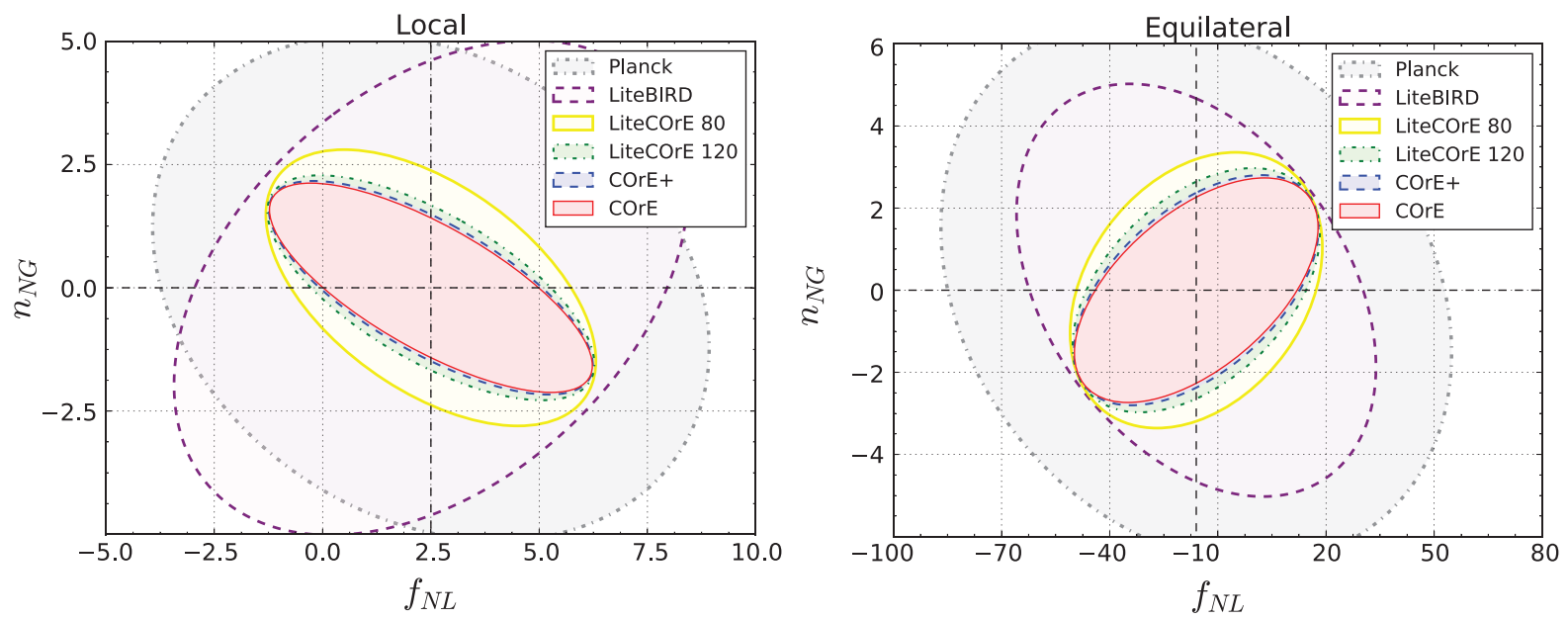

Figure 34: 1-sigma contours in the $f_{\mathrm{NL}}-n_{\mathrm{NG}}$ plane, for local (left panel) and equilateral (right panel) scale-dependent bispectra. Pivot scale $k_{P}=0.055 \mathrm{Mpc}^{-1}$.

features in the inflaton potential has the form

$$
B^{\text {feat }}\left(k_{1}, k_{2}, k_{3}\right)=6 A f_{\mathrm{NL}}^{\text {feat }} \cos \left[\omega\left(k_{1}+k_{2}+k_{3}\right)+\phi\right],
$$

where $A$ is the power spectrum amplitude, $\phi$ is a phase factor, and $\omega$ defines the oscillation 


\begin{tabular}{l|ccccc|ccccc} 
& \multicolumn{5}{|c|}{ independent } & \multicolumn{7}{|c}{ joint analysis } \\
& LC120 & C & C + & Planck & ideal & LC120 & C & C + & Planck & ideal \\
\hline T a,aa & 3.7 & $\mathbf{3 . 6}$ & 3.4 & $(5.4)$ & 2.7 & 40 & $\mathbf{3 9}$ & 38 & $(48)$ & 32 \\
T a,ai & 20 & $\mathbf{2 0}$ & 19 & $(29)$ & 15 & 290 & $\mathbf{2 8 0}$ & 280 & $(350)$ & 230 \\
T a,ii & 260 & $\mathbf{2 5 0}$ & 240 & $(360)$ & 190 & 3300 & $\mathbf{3 2 0 0}$ & 3200 & $(3800)$ & 2600 \\
T i,aa & 3.3 & $\mathbf{3 . 2}$ & 3.0 & $(4.7)$ & 2.4 & 47 & $\mathbf{4 6}$ & 45 & $(51)$ & 38 \\
T i,ai & 15 & $\mathbf{1 5}$ & 14 & $(21)$ & 11 & 150 & $\mathbf{1 5 0}$ & 150 & $(170)$ & 130 \\
T i,ii & 170 & $\mathbf{1 7 0}$ & 160 & $(230)$ & 130 & 1300 & $\mathbf{1 3 0 0}$ & 1300 & $(1400)$ & 1100 \\
\hline E a,aa & 4.5 & $\mathbf{4 . 2}$ & 3.9 & $(34)$ & 2.4 & 28 & $\mathbf{2 7}$ & 25 & $(150)$ & 18 \\
E a,ai & 6.9 & $\mathbf{6 . 4}$ & 5.9 & $(93)$ & 3.6 & 55 & $\mathbf{5 3}$ & 51 & $(620)$ & 37 \\
E a,ii & 61 & $\mathbf{5 8}$ & 53 & $(940)$ & 34 & 530 & $\mathbf{5 2 0}$ & 510 & $(3900)$ & 390 \\
E i,aa & 3.7 & $\mathbf{3 . 5}$ & 3.2 & $(27)$ & 2.0 & 27 & $\mathbf{2 6}$ & 25 & $(120)$ & 19 \\
E i,ai & 5.0 & $\mathbf{4 . 8}$ & 4.4 & $(62)$ & 2.8 & 74 & $\mathbf{7 3}$ & 72 & $(420)$ & 56 \\
E i,ii & 30 & $\mathbf{2 8}$ & 27 & $(460)$ & 19 & 140 & $\mathbf{1 4 0}$ & 140 & $(1600)$ & 110 \\
\hline T+E a,aa & 2.2 & $\mathbf{2 . 1}$ & 1.9 & $(4.9)$ & 1.4 & 9.8 & $\mathbf{9 . 5}$ & 9.2 & $(24)$ & 7.0 \\
T+E a,ai & 3.6 & $\mathbf{3 . 4}$ & 3.2 & $(22)$ & 2.3 & 19 & $\mathbf{1 9}$ & 18 & $(130)$ & 14 \\
T+E a,ii & 31 & $\mathbf{2 9}$ & 28 & $(230)$ & 20 & 220 & $\mathbf{2 2 0}$ & 220 & $(1200)$ & 170 \\
T+E i,aa & 1.8 & $\mathbf{1 . 8}$ & 1.6 & $(4.1)$ & 1.2 & 9.9 & $\mathbf{9 . 7}$ & 9.5 & $(24)$ & 7.4 \\
T+E i,ai & 2.8 & $\mathbf{2 . 7}$ & 2.5 & $(15)$ & 1.8 & 24 & $\mathbf{2 4}$ & 23 & $(74)$ & 19 \\
T+E i,ii & 16 & $\mathbf{1 5}$ & 15 & $(130)$ & 11 & 47 & $\mathbf{4 6}$ & 46 & $(430)$ & 37 \\
& & & & & & & & & &
\end{tabular}

Table 20: Same as Table 18, but for the neutrino velocity isocurvature mode.

\begin{tabular}{c|cccccc} 
& LiteCORE & LiteCORE & CORE & COrE + & Planck & LiteBIRD \\
& 80 & 120 & M5 & & & \\
\hline$f_{\text {NL local }}$ & 2.5 & 2.5 & $\mathbf{2 . 3}$ & 2.3 & 4.3 & 3.9 \\
$f_{\text {NL equil. }}$ & 23 & 22 & $\mathbf{2 1}$ & 21 & 47 & 33 \\
\hline$n_{\text {NG local }}$ & 1.9 & 1.5 & $\mathbf{1 . 4}$ & 1.4 & 3.4 & 3.3 \\
$n_{\text {NG }}$ equil. & 2.2 & 2.0 & $\mathbf{1 . 8}$ & 1.8 & 4.4 & 3.9
\end{tabular}

Table 21: Forecasts for the marginalized $1 \sigma f_{\mathrm{NL}}$ and $n_{\mathrm{NG}}$ error bars in scale-dependent NG models. We assumed $n_{\mathrm{NG}}=0$ as fiducial value for the $\mathrm{NG}$ spectral index (for both shapes), corresponding to scale invariance. For the amplitudes we took instead $f_{\mathrm{NL}}^{\text {fid,local }}=2.5, f_{\mathrm{NL}}^{\text {fid,equil. }}=-16$, which represent the current Planck central values.

frequency. Our forecasts for this shape are shown in Table 22. Also in this case as for standard LEO shapes, the final $S / N$ improvement with respect to Planck amounts to a factor $\approx 2$, is essentially independent of the specific CORE configuration considered and is mostly driven by improved polarization sensitivity.

The other type of feature considered has the form described in Section 2.3.3 from a transient reduction in the speed of sound away from $c_{s}=1$, with uninterrupted slow roll. This is part of a general class of effectively single-field models where the leading contribution to the bispectrum is given by the power spectrum feature and its first and second derivatives, with coefficients that 


\begin{tabular}{l|ccccc} 
& LiteCORE & LiteCORE & CORE & COrE + & Planck \\
& 80 & 120 & M5 & & \\
\hline $\mathrm{T}, \omega=50$ & 60 & 53 & $\mathbf{5 2}$ & 52 & 73 \\
$\mathrm{~T}, \omega=100$ & 87 & 86 & $\mathbf{8 5}$ & 85 & 102 \\
\hline $\mathrm{T}+\mathrm{E}, \omega=50$ & 21 & 21 & $\mathbf{2 0}$ & 20 & 42 \\
$\mathrm{~T}+\mathrm{E}, \omega=100$ & 26 & 25 & $\mathbf{2 4}$ & 24 & 51
\end{tabular}

Table 22: Forecasts for $1 \sigma$ error bars on the $f_{\mathrm{NL}}$ parameter for the feature model of Eq. (8.2). We consider two different oscillation frequencies and we take 0 as fiducial value for $\phi$.

are independent of scale, see Eq. (2.8).

The results in Table 23 are for the simplest case of a reduction in $c_{s}$ given by a Gaussian in $e$-folds. ${ }^{15}$ This leads to enveloped oscillations in the bispectrum (correlated with similar features in the power spectrum and higher correlation functions). The oscillations are linear in $k$ with a frequency determined by the instant $\left(\eta_{0}\right)$ of maximum reduction in $c_{s}$. An important difference with the previous oscillating shape is that here the phase depends on the $k$-triangle: oscillations in the equilateral shape are $\pi / 2$ out of phase with respect to the ones in the squeezed limit. The oscillations are modulated and the envelope is largest away from $k=0$ [301].

\subsubsection{Trispectrum}

So far we have discussed only the bispectrum parameters $f_{\mathrm{NL}}$, but interesting information is also contained in the angular trispectrum (4-point function in harmonic space; see $[52,302,303]$ and references therein).

To get an idea of future improvements in this case, we forecasted the expected performance of CORE to constrain the $g_{\mathrm{NL}}$ local trispectrum parameter using T-only and E-only trispectra, as shown in Table 24. If we consider temperature-only data (TTTT trispectrum), we find that CORE can improve on current error bars from Planck by a factor $\sim 3$ from the current $\sigma_{g_{\mathrm{NL}}}=7.7 \times 10^{4}$ (Planck) constraint [52] to $\sigma_{g_{\mathrm{NL}}}=2.8 \times 10^{4}$ (CORE). However, this mostly comes from the fact that the error bar for Planck refers to the actual data analysis, with $\ell_{\max }=1600$ (imperfect knowledge of the noise model did not allow to go to higher multipoles with Planck so far for the trispectrum), while the CORE Fisher matrix forecast has $\ell_{\max }=3000$. If we consider the polarization-only $E E E E$ trispectrum, we see, in line with the $E E E$ bispectrum forecasts, that CORE will allow for massive improvements compared to Planck. The trispectrum E-only error bars shrink by a factor $\sim 20$, from $\sigma_{g_{\mathrm{NL}}} \sim 5 \times 10^{5}$ (Planck at $\ell_{\max }=1600$; note that only the TTTT trispectrum was actually analyzed with Planck data, so this is a forecast) to $\sigma_{g_{\mathrm{NL}}} \sim 2.5 \times 10^{4}$ $\left(\mathrm{CORE}, \ell_{\max }=3000\right)$. We find that different CORE configurations (LiteCORE-120, CORE, COrE+) perform nearly identically.

\subsection{Other methods}

Two interesting and potentially powerful new approaches have been proposed over the past few years to extract NG information: one using observations of the cosmic infrared background (CIB),

\footnotetext{
${ }^{15}$ More precisely, a Gaussian in $u=1-c_{s}^{-2}$ away from zero, $u=-u_{\max } \exp \left(-\beta\left(N-N_{0}\right)^{2}\right)=$ $-u_{\max } \exp \left(-\beta\left(\log \left(\eta / \eta_{0}\right)\right)^{2}\right)$, where $\eta$ is the conformal time [300]. The rate of change of $c_{s}$ is given by $s \equiv \dot{c}_{s} /\left(c_{s} H\right)$.
} 


\begin{tabular}{|c|c|c|c|c|c|}
\hline \multicolumn{6}{|c|}{$\mathbf{T},|s|_{\max }=0.1$} \\
\hline$-\eta_{0}$ & Planck & LiteCORE-80 & LiteCORE-120 & CORE & $\mathrm{COrE}+$ \\
\hline 30 & $2.5 \times 10^{-6}$ & $7.1 \times 10^{-7}$ & $3.6 \times 10^{-7}$ & $3.5 \times 10^{-7}$ & $3.5 \times 10^{-7}$ \\
\hline 60 & $1.7 \times 10^{-5}$ & $1.2 \times 10^{-5}$ & $8.8 \times 10^{-6}$ & $8.8 \times 10^{-6}$ & $8.8 \times 10^{-6}$ \\
\hline \multicolumn{6}{|c|}{$\mathbf{T}+\mathbf{E},|s|_{\max }=0.1$} \\
\hline$-\eta_{0}$ & Planck & LiteCORE-80 & LiteCORE-120 & CORE & $\mathrm{COrE}+$ \\
\hline 30 & $2.0 \times 10^{-6}$ & $2.6 \times 10^{-7}$ & $1.6 \times 10^{-7}$ & $1.4 \times 10^{-7}$ & $1.4 \times 10^{-7}$ \\
\hline 60 & $1.2 \times 10^{-5}$ & $4.0 \times 10^{-6}$ & $3.6 \times 10^{-6}$ & $3.3 \times 10^{-6}$ & $3.3 \times 10^{-6}$ \\
\hline \multicolumn{6}{|c|}{$\mathbf{T},|s|_{\max }=0.2$} \\
\hline$-\eta_{0}$ & Planck & LiteCORE-80 & LiteCORE-120 & CORE & $\mathrm{COrE}+$ \\
\hline 30 & $4.4 \times 10^{-7}$ & $9.3 \times 10^{-8}$ & $2.5 \times 10^{-8}$ & $2.5 \times 10^{-8}$ & $2.5 \times 10^{-8}$ \\
\hline 60 & $8.0 \times 10^{-7}$ & $3.6 \times 10^{-7}$ & $2.4 \times 10^{-7}$ & $2.4 \times 10^{-7}$ & $2.4 \times 10^{-7}$ \\
\hline \multicolumn{6}{|c|}{$\mathbf{T}+\mathbf{E},|s|_{\max }=0.2$} \\
\hline$-\eta_{0}$ & Planck & LiteCORE-80 & LiteCORE-120 & CORE & $\mathrm{COrE}+$ \\
\hline 30 & $3.9 \times 10^{-7}$ & $3.6 \times 10^{-8}$ & $1.3 \times 10^{-8}$ & $1.1 \times 10^{-8}$ & $1.1 \times 10^{-8}$ \\
\hline 60 & $6.4 \times 10^{-7}$ & $1.3 \times 10^{-7}$ & $1.2 \times 10^{-7}$ & $1.1 \times 10^{-7}$ & $1.1 \times 10^{-7}$ \\
\hline
\end{tabular}

Table 23: Forecasts for the $1 \sigma$ error bars of the intensity $|u|_{\max }$ of a Gaussian-shaped transient reduction in the speed of sound of the inflaton, for different combinations of the maximum change rate $|s|_{\max }$ and the instant of maximum reduction $\eta_{0}$, which is also the oscillation frequency of the feature, equal to $\omega$ in Eq. (8.2). Typical expected values for $|u|_{\max }$ are $\mathcal{O}\left(10^{-2}-10^{-1}\right)$. In general, CORE's high signal-to-noise polarization data shrinks the error bars by a few units. For low values of $-\eta_{0}$ (e.g. $-\eta_{0}=30$ ), the feature peaks in intensity at high $\ell$; this makes the shrinkage of the error bars even more dramatic, since it is also driven by the increased effective $\ell_{\max }$ of CORE, especially for LiteCORE-120 and higher configurations.

\begin{tabular}{l|cccc}
$\sigma_{\mathrm{g}_{\mathrm{NL}}} \times 10^{4}$ & LiteCORE-120 & CORE & COrE + & Planck \\
\hline TTTT & 2.8 & $\mathbf{2 . 8}$ & 2.8 & $(7.7)$ \\
EEEE & 2.8 & $\mathbf{2 . 7}$ & 2.6 & 50
\end{tabular}

Table 24: Forecast $g_{\mathrm{NL}}$ standard deviations for different experiments.

and the other based on spectral distortions created through energy release in the early universe [304-308]. Both these methods work only for a specific class of bispectrum (trispectrum) shapes, namely those peaking in the so-called squeezed limit (i.e., bispectrum or trispectrum configurations in which one wavenumber is much smaller than the others). This happens notably for the local shape, which is of particular interest since it allows one to discriminate between single-field and multiple-field inflation. On the other hand, a complete study of primordial NG must include a large number of shapes, the vast majority of which does not peak in the squeezed limit (e.g., equilateral and folded shapes, as well as many other cases characterized by breaking of scale-invariance or isotropy; see also previous Section). For those scenarios, a direct estimate of temperature and 
polarization angular bispectra (and trispectra) is the only way forward.

The first method was recently considered in [309]. It is based on exploiting primordial NG signatures (i.e. scale-dependent bias on very large scales) in the CIB power spectrum. In this case, the main obstacle is represented by dust contamination. However, dust contamination is also the main issue to overcome for the primordial $B$-mode analysis. Therefore many high-frequency channels are planned in future surveys, and the achievable level of foreground subtraction should make CIB-based local $f_{\mathrm{NL}}$ measurements very promising, as originally pointed out in [309]. This is shown here explicitly for CORE, which, according to our forecasts, will be able to achieve $f_{\mathrm{NL}}^{\text {local }}<1$ sensitivity with this probe.

The second method consists of extracting $f_{\mathrm{NL}}$ via measurements of correlations between CMB temperature and $\mu$-type spectral distortion ${ }^{16}$ anisotropies generated by dissipation of primordial acoustic waves [310-315]. Interestingly, such measurements do not require absolute calibration, as they do not make direct use of the $\mu$-distortion monopole. However, an interpretation of the data in terms of $f_{\mathrm{NL}}^{\text {local }}$ relies on knowledge of the average dissipation-induced $\mu$-distortion, such that a combination with an absolute spectrometer (e.g., PIXIE) is necessary [316].

\subsubsection{CIB power spectrum}

The angular power spectrum of the cosmic infrared background (CIB) is another sensitive probe of the local primordial bispectrum (and potentially of other bispectra peaking in the squeezed limit). CIB measurements are integrated over a large volume so that the scale-dependent bias from the primordial non-Gaussianity leaves a strong signal in the CIB power spectrum. Although galactic dust dominates over the non-Gaussian CIB signal, it is possible to mitigate the dust contamination with enough frequency channels, especially if high frequencies such as the Planck $857 \mathrm{GHz}$ channel are available.

We adopt here a Fisher matrix approach to investigate the sensitivity of CORE and COrE + to $f_{\mathrm{NL}}$ through measurements of the CIB power spectrum. The Fisher matrix elements can be written as

$$
F_{i j}=\sum_{\ell=\ell_{\min }}^{\ell_{\max }} \frac{2 \ell+1}{2} f_{\text {sky }} \operatorname{Tr}\left(\mathbf{C}_{\ell}^{-1} \frac{\partial \mathbf{C}_{\ell}}{\partial \theta_{i}} \mathbf{C}_{\ell}^{-1} \frac{\partial \mathbf{C}_{\ell}}{\partial \theta_{j}}\right),
$$

where the elements of the covariance matrix $\mathbf{C}_{\ell}$ are defined as the auto- and cross-power spectra of data at $N_{\nu}$ different observational frequencies, computed on a fraction of the sky $f_{\text {sky }}$. Model parameters $\theta$ include $f_{\mathrm{NL}}$, the CIB model parameters (see $[309,317]$ ) plus the parameters related to the Galactic dust emission. We refer the reader to [309] for all technical details and for the full calculation of the CIB power spectrum in the presence of primordial NG.

The Galactic dust emission is the main contaminant for the detection of CIB anisotropies on large angular scales, where it is orders of magnitude brighter than the CIB. Distinguishing Galactic from extragalactic dust emission is especially difficult because of their fairly similar spectral energy distribution (SED) which approximately scales in both cases like a modified blackbody law. Extracting the CIB signal from Galactic contamination thus requires a very accurate component separation. Here we assume that CIB maps can be reconstructed by linearly combining the set of

\footnotetext{
${ }^{16} \mathrm{~A} \mu$-type distortion is created by energy release at redshifts $5 \times 10^{4} \lesssim z \lesssim 2 \times 10^{6}$, when Comptonization is very efficient [305-307].
} 


\begin{tabular}{|c|c|c|c|c|c|c|c|c|c|}
\hline & \multicolumn{8}{|c|}{$\mathrm{COrE}+$} & $\sigma\left(\mathbf{f}_{\mathrm{NL}}\right)$ \\
\hline$\nu[\mathrm{GHz}]$ & 220 & 255 & 295 & 340 & 390 & 450 & 520 & 600 & \\
\hline fwhm [arcmin] & 3.82 & 3.29 & 2.85 & 2.47 & 2.15 & 1.87 & 1.62 & 1.40 & \\
\hline $\mathrm{w}^{-1}\left[\mathrm{Jy}^{2} \mathrm{sr}^{-1}\right]$ & 0.654 & 1.43 & 5.20 & 8.31 & 13.50 & 22.98 & 39.88 & 69.26 & \\
\hline \multirow[t]{2}{*}{$\Sigma_{\mathrm{CIB}}^{2}\left[\mathrm{Jy}^{2} \mathrm{sr}^{-1}\right]$} & - & 8.04 & - & 47.3 & - & 212. & 382. & - & 1.6 \\
\hline & \multicolumn{8}{|c|}{ COrE + with Planck } & \\
\hline \multirow[t]{2}{*}{$\Sigma_{\text {CIB }}^{2}\left[\mathrm{Jy}^{2} \mathrm{sr}^{-1}\right]$} & - & 1.97 & - & 10.8 & - & 45.6 & 90.2 & 163.6 & 0.6 \\
\hline & \multicolumn{8}{|c|}{ CORE } & $\sigma\left(\mathbf{f}_{\mathrm{NL}}\right)$ \\
\hline$\nu[\mathrm{GHz}]$ & 220 & 255 & 295 & 340 & 390 & 450 & 520 & 600 & \\
\hline fwhm [arcmin] & 5.23 & 4.57 & 3.99 & 3.49 & 3.06 & 2.65 & 2.29 & 1.98 & \\
\hline $\mathrm{w}^{-1}\left[\mathrm{Jy}^{2} \mathrm{sr}^{-1}\right]$ & 0.29 & 0.57 & 0.77 & 1.08 & 2.16 & 3.55 & 6.2 & 11.0 & \\
\hline \multirow[t]{2}{*}{$\Sigma_{\text {CIB }}^{2}\left[\mathrm{Jy}^{2} \mathrm{sr}^{-1}\right]$} & - & 1.8 & - & 8.8 & - & 42.9 & 80.1 & - & 0.7 \\
\hline & \multicolumn{8}{|c|}{ CORE with Planck } & \\
\hline$\Sigma_{\text {CIB }}^{2}\left[\mathrm{Jy}^{2} \mathrm{sr}^{-1}\right]$ & - & 1.03 & - & 5.2 & - & 23.3 & 43.9 & 68.9 & 0.34 \\
\hline
\end{tabular}

Table 25: Instrumental specifications of $\mathrm{COrE}+$ and $\mathrm{CORE}$, the noise variance in the reconstructed CIB maps $\left(\Sigma_{\mathrm{CIB}}^{2}\right)$ and the uncertainty on $f_{\mathrm{NL}}$ (assuming $\left.f_{\mathrm{NL}}=0\right)$.

frequency maps (at $\nu>200 \mathrm{GHz}$ and after subtracting $\mathrm{CMB}$ ) on the basis of the "mixing" matrix A that describes the frequency dependence of the sky signal components [168]. If observations are available at $n$ frequencies, we assume that CIB maps can be reconstructed only in $N_{\nu} \sim n / 2$ frequencies, while the other channels are dedicated to the estimation of the mixing matrix and the Galactic dust template used in the subtraction. This can be seen as a general approach, independent of the specific component separation method employed. The auto- and cross-power spectra in clean CIB maps then read as

$$
C_{\ell}^{\nu_{i} \nu_{j}}=C_{\ell}^{(\mathrm{CIB})}\left(\nu_{i}, \nu_{j}\right)+\varepsilon C_{\ell}^{d}\left(\nu_{i}, \nu_{j}\right)+\Sigma_{\mathrm{CIB}}^{2}\left(\nu_{i}\right) \delta_{i j},
$$

where $\Sigma_{\text {CIB }}$ is the noise variance and $\varepsilon$ is the fraction of the total Galactic dust power spectrum left over in the reconstructed CIB maps (we use as reference value $\varepsilon=10^{-2}$ ). The noise variance in the maps will be degraded after the component separation according to the frequency spectrum of the sky signals and the noise in the channels involved in the foreground subtraction [168, 318]. In our case, the noise degradation can be very severe due to the similar SED of the CIB and the dust emission, and frequencies much larger than $300 \mathrm{GHz}$ are mandatory to accurately separate them. Regarding the residual dust emission, we model the power spectrum $C_{\ell}^{d}$ as a power law, whose amplitude and slope are free parameters determined directly from the data. The SED of the dust emission is instead assumed to be perfectly known.

In Table 25 we report the uncertainty on $f_{\mathrm{NL}}, \sigma\left(f_{\mathrm{NL}}\right)$, estimated by the Fisher analysis for the $\mathrm{COrE}+$ and CORE configurations, assuming $f_{\mathrm{NL}}=0$ and a usable sky fraction of $40 \% . \sigma\left(f_{\mathrm{NL}}\right)$ is computed after marginalizing over all the other model parameters. Only frequencies higher than $200 \mathrm{GHz}$ are considered in the analysis. Channels at lower frequencies are in fact dominated by the $\mathrm{CMB}$ and should be dedicated to removing $\mathrm{CMB}$ fluctuations from the signal. Given the 8 frequency channels of CORE and $\mathrm{COrE}+$ at $\nu>200 \mathrm{GHz}$, we assume that clean CIB maps can 
be obtained in 4 of them. We can note in Table 25 that, after the component separation, the noise variance in these maps $\left(\Sigma_{\mathrm{CIB}}\right)$ increases by a factor of 3-12 with respect to the original noise. This significant degradation in sensitivity is due to the frequency coverage of CORE and COrE + , which does not include the wavelengths at which the SEDs of the dust and CIB mostly differ (i.e. at $\nu \gtrsim 800 \mathrm{GHz}$ ).

We see that the results for CORE here are actually better than those for COrE + . In the analysis we have assumed a maximum multipole of $\ell_{\max }=1000$. This choice guarantees that the shot-noise contribution from star-forming dusty galaxies is negligible. The resolution of the CORE experiment therefore is not a critical point to constrain $f_{\mathrm{NL}}$ using the CIB. While COrE + has a smaller beam, CORE has a higher sensitivity, and that is more important in this analysis. We find that $\left|f_{\mathrm{NL}}\right|=5$ would be measured by CORE at a $\sim 7 \sigma$ level $(\sim 3 \sigma$ for COrE + ), and $\left|f_{\mathrm{NL}}\right|$ of 1-2 would also be detectable with CORE with a significance of about $1-3 \sigma$. Achieving $\sigma\left(f_{\mathrm{NL}}\right) \sim 1$ for $\mathrm{COrE}+$ would require a more challenging dust removal at the order of 0.1 percent. We also find that the best results are for sky fractions around 0.4-0.6. This is already a significant improvement with respect to Planck that is expected to provide an upper limit on $f_{\mathrm{NL}}$ of $\sim 3.5$ at $1 \sigma$ [309].

However, the sensitivity of CORE and COrE + to the $f_{\mathrm{NL}}$ parameter can strongly improve when the highest Planck channels are taken into account and combined with CORE/COrE + data. Extending the frequency coverage to $857 \mathrm{GHz}$ is key to better separate $\mathrm{CIB}$ and dust emission. The noise degradation in the reconstructed CIB maps is significantly reduced when Planck channels are included in the mixing matrix, even more for $\mathrm{COrE}+$ than for $\mathrm{CORE}$. Under the hypothesis of $5 \mathrm{CORE} / \mathrm{COrE}+$ channels dedicated to the $\mathrm{CIB}$, we find $\sigma\left(f_{\mathrm{NL}}\right) \approx 0.6$ for $\mathrm{COrE}+$ and $\sigma\left(f_{\mathrm{NL}}\right) \approx 0.3$ for CORE, the latter allowing a $3 \sigma$ detection of $f_{\mathrm{NL}}$ down to values of 1 . In this configuration the residual dust emission is efficiently separated from the CIB fluctuations, and reducing the level of the dust residual in the CIB maps does not give significant improvements in the $f_{\mathrm{NL}}$ detection.

In summary, as anticipated at the beginning of this Section, the important conclusion of this analysis is that the combination of CORE and Planck should be able to reach a sensitivity $\sigma\left(f_{\mathrm{NL}}\right)<1$ with this probe, for the local shape.

\subsubsection{Spectral distortions}

The dissipation of acoustic modes set up by inflation in the early universe creates a small spectral distortion of the CMB [319-322]. Spatial fluctuations in the dissipation rate caused by primordial non-Gaussianity can enhance the amplitude of distortion anisotropies. It has been shown that this effect can, in principle, be used to estimate local $f_{\mathrm{NL}}$ from measurements of the $T$ - $\mu$ power spectrum, potentially leading to improvements up to many orders of magnitude over current constraints [310]. However, generally this requires futuristic levels of sensitivity (due to the necessity of measuring $\mu$ anisotropies with high accuracy), well beyond those achievable with a CORE-like survey. On the other hand, a strongly enhanced signal in the squeezed limit is expected for a specific class of models, characterized by excited initial states [323, 324]. It is interesting to consider such models using this technique with the next generation surveys like CORE. Therefore, we forecast here the detectability of a $C_{\ell}^{\mu T}$ signal in these non-Bunch-Davies (NBD) scenarios. 

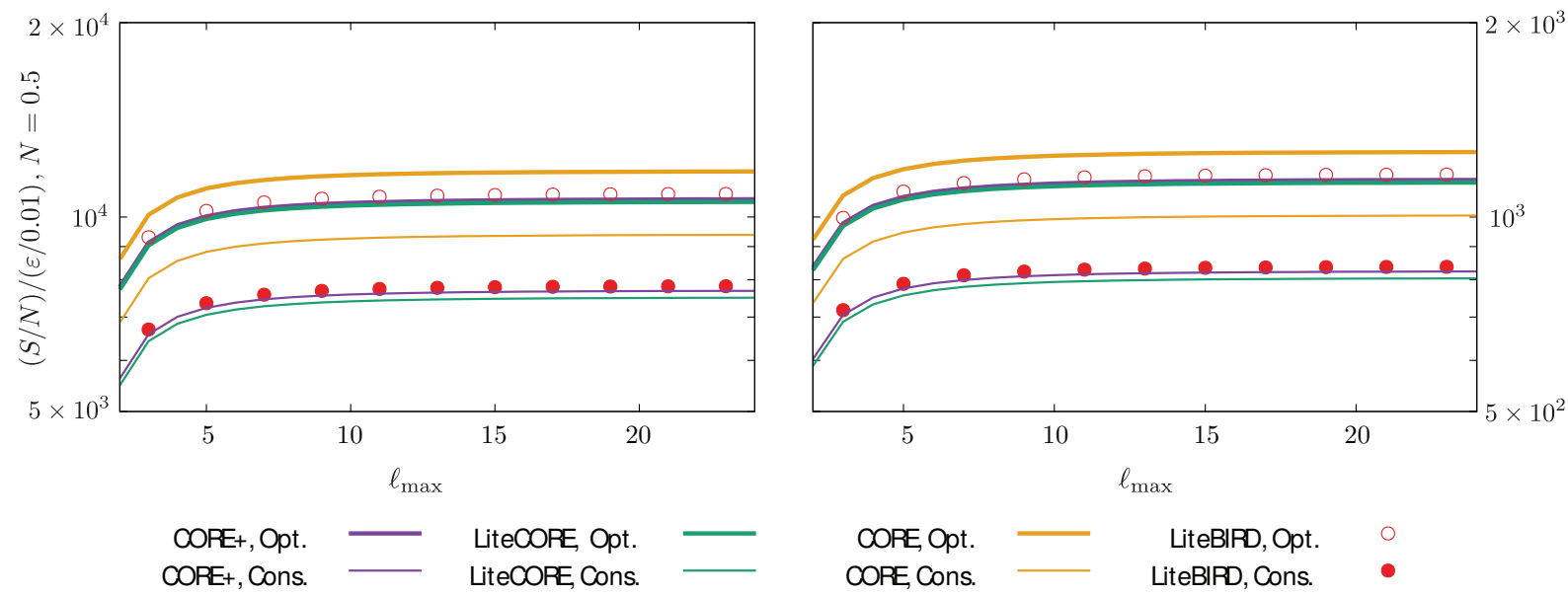

Figure 35: Signal-to-noise ratio of $C_{\ell}^{\mu T}$ from a modified initial state as a function of $\ell_{\max }$ for a fixed occupation number $N=0.5$. In our forecast we considered a 'conservative' and an 'optimistic' case. In the optimistic case we computed the signal-to-noise ratio by coadding the 4 lowest noise combinations of frequencies in the range $[80,200] \mathrm{GHz}$ that can be obtained from the LiteCORE, COrE + and CORE configurations. For COrE + we use $\Delta T=9.1 \mu \mathrm{K}$ arcmin, $\mathrm{FWHM}=10.5 \operatorname{arcmin}$ at $80 \mathrm{GHz}$ and $\Delta T=6.5$ $\mu \mathrm{K}$ arcmin, $\mathrm{FWHM}=9.3$ arcmin at $90 \mathrm{GHz}$. All other channels and experimental configurations are shown in the Tables of Section 3. In the conservative case we instead take only the best couple of frequencies for each configuration. This choice is justified by the fact that the issues of component separation and relative calibration of channels are not accounted for in this type of analysis. Therefore it is not obvious that all the channels which are assumed clean for standard temperature analysis will also be available for $T-\mu$ measurements. We see that the signal saturates in the first few multipoles. For each configuration of the satellite are shown models with both $\theta_{k}=$ const (left) and $\theta_{k} \approx k \eta_{0}$ (right). LiteCORE-80 and LiteCORE-120 perform essentially in the same way, due to the saturation of the signal after the first few multipoles, and are described by a single line. $S / N$ for Planck is a factor $\approx 100$ lower than for CORE.

Our results are obtained by closely following the methodology originally proposed in [312], to which we refer the reader for details.

The signal-to-noise ratio now reads

$$
\left(\frac{S}{N}\right)^{2}=\sum_{\ell}(2 \ell+1) \frac{\left(C_{\ell}^{\mu T}\right)^{2}}{C_{\ell}^{T T} C_{\ell}^{\mu \mu}}
$$

where large-scale temperature data are completely signal dominated, while the $\mu \mu$ spectrum is dominated by noise, for the experiments under study. Maps of $\mu$-anisotropies can be created from differences of temperature maps at different frequencies, $\nu_{1}$ and $\nu_{2}$, and the corresponding noise is computed as:

$$
C_{\ell}^{\mu \mu, N}=\Omega_{\mathrm{pix}} b_{\ell}^{-2}\left[\frac{\frac{\nu_{1} \nu_{2}}{\nu_{1}-\nu_{2}}}{56.80 \mathrm{Ghz}}\right]^{2}\left[\left(\sigma_{\mathrm{pix}}^{\nu_{1}}\right)^{2}+\left(\sigma_{\mathrm{pix}}^{\nu_{2}}\right)^{2}\right]
$$

In this formula, $\Omega_{\text {pix }}$ represents the solid angle (in steradians) subtended by a given pixel, $b_{\ell}$ is the beam and $\sigma_{\text {pix }}^{X}$ is the noise per pixel in channel X.

The initial conditions, set at a finite conformal time $\eta_{0}$, can be effectively parameterized through the occupation number of excited states, $N$, and a phase, $\theta_{k}$. The $C_{\ell}^{\mu T}$ are then computed 


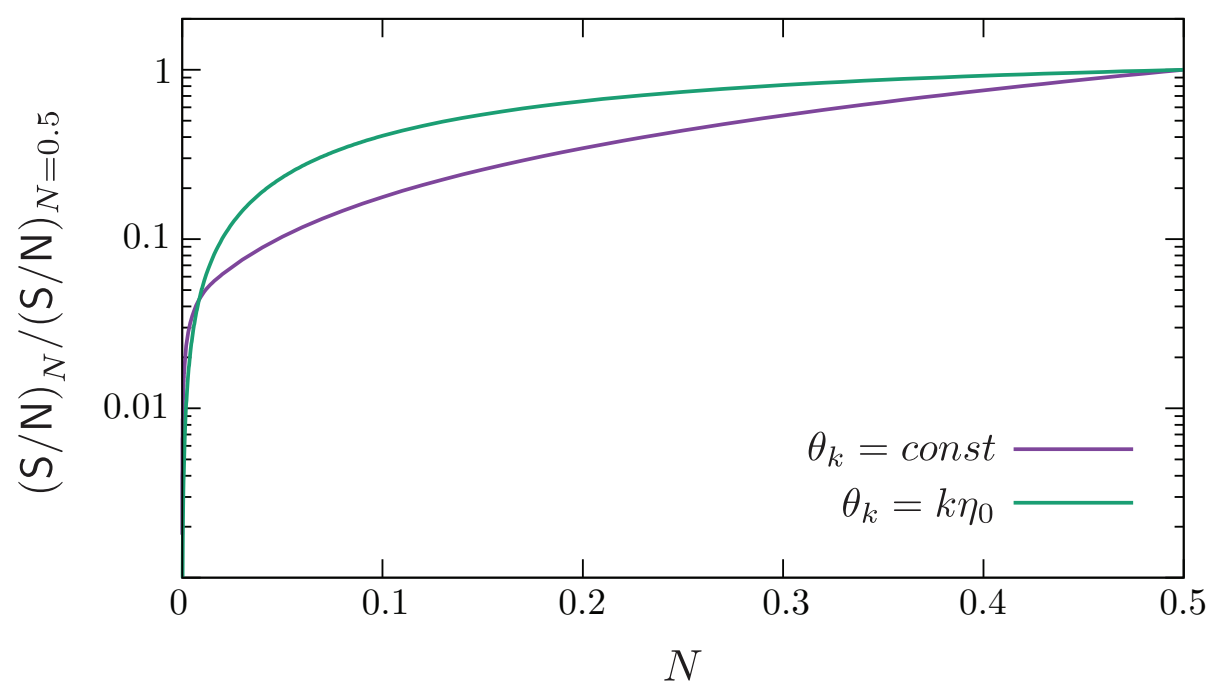

Figure 36: Dependence of the signal-to-noise ratio on the value of the occupation number $N$. Signal-tonoise ratio for different $N$ is here normalized to the values obtained for $N=0.5$ as displayed in Fig. 35 .

for two different parameterizations of the phase: $\theta_{k}=$ constant or $\theta_{k} \approx k \eta_{0}$. The final signalto-noise results, displayed in Figs. 35 and 36, show (in agreement with similar findings in [312], which referred to a PIXIE-like experiment) that NBD models can be probed by CORE with high statistical significance using this test. More specifically, in Fig. 35 we show the signal-to-noise ratio of $C_{\ell}^{\mu T}$ from a modified initial state as a function of $\ell_{\max }$ for a fixed occupation number $N=0.5$, slow-roll parameter $\epsilon=0.01$, and for both parameterizations of $\theta_{k}$. We see that all experimental configurations achieve a very high signal-to-noise. In all the considered cases we obtain $S / N>500$. By comparison, direct $f_{\mathrm{NL}}$ measurements for these models, based on bispectrum template fitting, achieve a sensitivity $\sigma_{\mathrm{fNL}} \approx 5$, which would make the case plotted in Fig. 35 undetectable. We also see that for this test, CORE outperforms the other configurations, including $\mathrm{COrE}+$, due to better sensitivity at $80 \mathrm{GHz}$. Fig. 36 shows how the signal-to-noise changes when varying the occupation number $N$. Results are normalized to the values shown in Fig. 35 for $N=0.5$. Unless occupation numbers become very low, the signal should remain detectable.

Interestingly, Planck is already expected to have a strong discriminating power for these models, although with a signal-to-noise ratio a factor roughly 100 below CORE. On the other hand, we have to stress that systematic sources of error are not taken into account here. The expected two orders of magnitude improvement of CORE over Planck looks therefore even more important and reassuring, for the purpose of retaining a high signal-to-noise after relative calibration error and foreground subtraction are taken into account.

\subsection{Summary}

In this Section we considered NG signatures produced by many different inflationary scenarios. We forecasted the detectability of these signatures with CORE-type surveys in terms of future bounds on the NG parameters $f_{\mathrm{NL}}$. For standard local, equilateral, and orthogonal (LEO) shapes, we find that CORE will allow for improvements of a factor between 2 and 3 over current Planck 
bounds, giving $1 \sigma f_{\mathrm{NL}}$ error forecasts of about 2, 20, and 10 for the local, equilateral, and orthogonal shapes, respectively (see Table 17). These forecasts are obtained using direct bispectrum measurements with the same methods used to analyze the Planck data. Similar improvements are also expected for the $g_{\mathrm{NL}}$ trispectrum constraints, for the $n_{\mathrm{NG}}$ constraints in models with a mild running of $f_{\mathrm{NL}}$, and for the $f_{\mathrm{NL}}$ constraints in the case of oscillatory bispectra. This improvement shrinks the available volume in LEO bispectrum space by a factor of $\approx 20$ with respect to Planck. Our CMB bispectrum-based constraints are slightly worse than recent $f_{\mathrm{NL}}$ forecasts using the bispectrum of forthcoming galaxy surveys, like Euclid, at least for the local shape (see e.g. [325-328]). However, LSS bispectrum measurements must correctly and reliably account for complex non-primordial NG contamination arising from non-linear gravitational evolution of structure (such as bias), making the CMB a much cleaner probe. Non-primordial LSS non-linearities seem moreover more problematic in the equilateral limit. Consequently, the CMB might provide the only way to make progress for many nonlocal shapes (see e.g. [329]) if we exclude futuristic 21-cm measurements [330].

Looking at other NG tests not based on direct bispectrum estimation, we find a very interesting result: CORE should be able to achieve $f_{\mathrm{NL}} \approx 1$ sensitivity for the local shape by measuring the scale-dependent bias in the CIB power spectrum on large scales. (Note that these $f_{\mathrm{NL}} \mathrm{CIB}$ forecasts account for the dust subtraction explicitly.) This is a crucial threshold for discriminating between single and multi-field inflation. Such a sensitivity would be comparable or better than what is achievable by estimating $f_{\mathrm{NL}}^{\text {local }}$ from scale-dependent bias in future galaxy surveys.

If we move beyond 'standard' LEO bispectra, we find that CORE can achieve massive improvements over current constraints for specific targeted models such as isocurvature NG, where $S / N$ improvements by an order of magnitude are expected via bispectrum estimation, and for some NBD models, where of order $\approx 100$ improvements in sensitivity can be achieved via measurements of cross-correlations between temperature and $\mu$-distortion anisotropies. The combination of CORE with an absolute spectrometer such as PIXIE [316] would allow a model-independent interpretation of the $C_{\ell}^{\mu T}$ constraints. Moreover, CORE should detect the lensing-ISW bispectrum with almost an order of magnitude better signal-to-noise than Planck. For most of the performed bispectrum tests, we find that the LiteCORE, CORE, and COrE + configurations are nearly equivalent in terms of final $f_{\mathrm{NL}}$ sensitivity, with CORE falling in between LiteCORE and COrE + . The CIB and $\mathrm{T} \mu$ tests, on the other hand, favor CORE over the other configurations due to its higher sensitivity.

\section{Topological defects}

In this Section we will take Abelian Higgs gauge cosmic strings (as are present for example in Dterm hybrid inflation) as a representative topological defect. The power spectra coming from gauge strings and other defects are relatively similar, though the precise shape and normalization are different [331]. However, the procedure for searching for strings and other defects is in principle the same. In the next Subsection we will briefly introduce how the CMB power spectra from defects is obtained before we describe the statistical analysis performed to assess the sensitivity of the different missions to either detect defects or to distinguish them from primordial gravitational waves. 


\subsection{Calculation of CMB from defects}

A general method for calculating CMB power spectra from defects starts with the numerical solution of the classical field theory in an expanding background, from which the unequal time correlators (UETCs) of the energy-momentum tensor are extracted [332, 333]. One can also model the defect sources in various computationally less expensive ways [334-336], but these effective models must be checked against large-scale numerical simulations.

Using rotational symmetry, it can be shown that there are only five independent correlators: three scalar, one vector, and one tensor. The three scalar correlators can be thought of as sources for the auto- and cross-correlation of the two Bardeen potentials $\Phi$ and $\Psi$. Unlike in the case of inflationary perturbations, the vector perturbations coming from defects do not die out, as they are constantly being seeded, and they contribute (together with the tensor modes) to create a B-mode polarization signal.

The correlation functions can then be diagonalized and their eigenfunctions used as sources for an Einstein-Boltzmann solver. The CMB power spectra from each eigenfunction can finally be summed.

For parameterizing defect power spectra, it is convenient to define the dimensionless parameter

$$
G \mu=2 \pi G \phi_{0}^{2}
$$

where $\phi_{0}$ is the expectation value of the symmetry-breaking field (assumed complex) and $\mu$ is the cosmic string tension in models with strings. Power spectra are proportional to $(G \mu)^{2}$. It is also convenient to parameterize the fractional contribution of the defects to the CMB temperature power spectrum $C_{\ell}$ by

$$
f_{10}=\frac{C_{10}^{\text {def }}}{C_{10}^{\text {inf }}+C_{10}^{\text {def }}}
$$

where $C_{\ell}^{\text {def }}$ is the power spectrum produced by defects and $C_{\ell}^{\text {inf }}$ the power spectrum from the underlying inflationary model. The two sets of fluctuations are statistically independent. Note that all different power spectra (temperature and polarization) are linked in the sense that for a given defect model, the normalization of one forces the normalization of the others. There is no freedom to normalize them independently. Therefore, even though $f_{10}$ is defined for the temperature power spectrum, it also gives the normalization of the polarization power spectra. Note also that the constant of proportionality relating $f_{10}$ and $(G \mu)^{2}$ varies between kinds of topological defects, but not by more than a factor of a few. Constraints expressed in terms of $f_{10}$ are similar for different kinds of defects, reflecting the similarity of the shape of the power spectra. The power spectra from the most recent computations for cosmic strings [337, 338] are shown in Fig. 37.

\subsection{MCMC Fits}

In order to quantify the ability of future satellite CMB missions to constrain the abundance of cosmic strings in the universe, we adopt a MCMC approach as in previous works [339, 340]. Our parameters are the standard six $\Lambda$ CDM parameters $\left\{\omega_{b}, \omega_{c}, \theta_{\mathrm{MC}}, \tau, \ln 10^{10} A_{s}, n_{s}\right\}$ with the addition of the scalar to tensor ratio $r$ and/or the string amplitude $10^{12}(G \mu)^{2}$. We use flat priors in these parameters that are always wide enough to encompass the posteriors, and impose the 


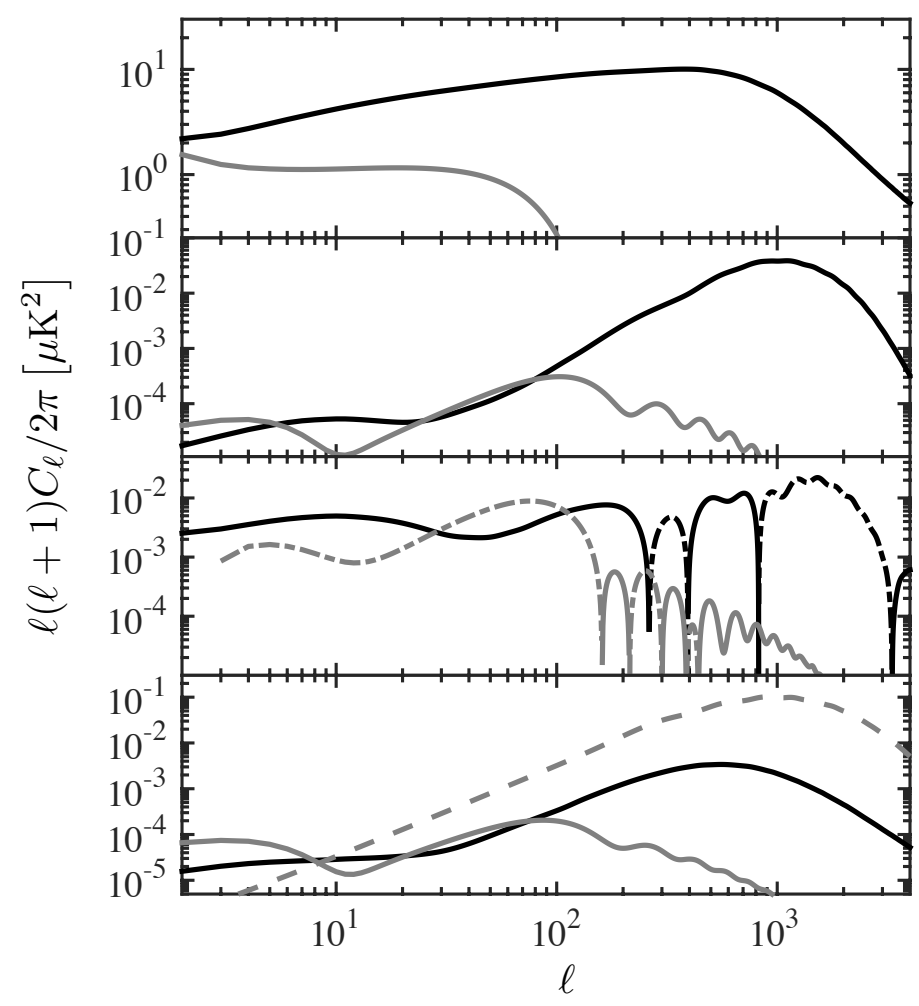

Figure 37: CMB perturbation power spectra from cosmic strings [338] with $f_{10}=5 \times 10^{-3}$ (solid black), compared to inflationary tensor modes with $r=3 \times 10^{-3}$ (solid grey) and lensing B-modes (grey dashed). Top to bottom are TT, EE, TE, and BB. Negative values in the TE power spectrum are shown as dotted lines. The signal from inflationary scalar perturbations in the TT, EE, and TE channels are not shown, as they are much larger.

condition $r \geq 0$ and $G \mu \geq 0$ when those parameters are varied (else they are set to zero). The flat prior on $(G \mu)^{2}$ translates (for small admixtures of cosmic strings) to a flat prior on the fractional contribution at $\ell=10 f_{10}$.

For Planck temperature and polarization data, the $95 \%$ upper limit on $f_{10}$ is about $1 \%$ [338]; strings are thus a small contribution. We are therefore justified in computing the string spectra only for a reference cosmological model, varying only the amplitude of the contribution as described above. The small errors in the shape of the power spectra will be insignificant.

Strings have an important B-mode signal, which is obscured by the lensing of the E-mode polarization of the dominant inflationary fluctuations. In the analysis we consider two different approaches to analyzing the B-mode polarization data, which span the range of success in disentangling the lensed B-modes from the signal from primordial gravitational waves $(r)$ or defects $(G \mu)$ :

1. Standard, where the full lensing signal is present;

2. Fully delensed, where the B-mode is entirely due to gravitational waves or defects. 
In Fig. 38 we show $1 \sigma$ and $2 \sigma$ likelihood contours for the $r-(G \mu)^{2}$ posterior, marginalized over all other parameters, for the fully delensed case. The $95 \%$ upper limits for all cases considered here are listed in Table 26.

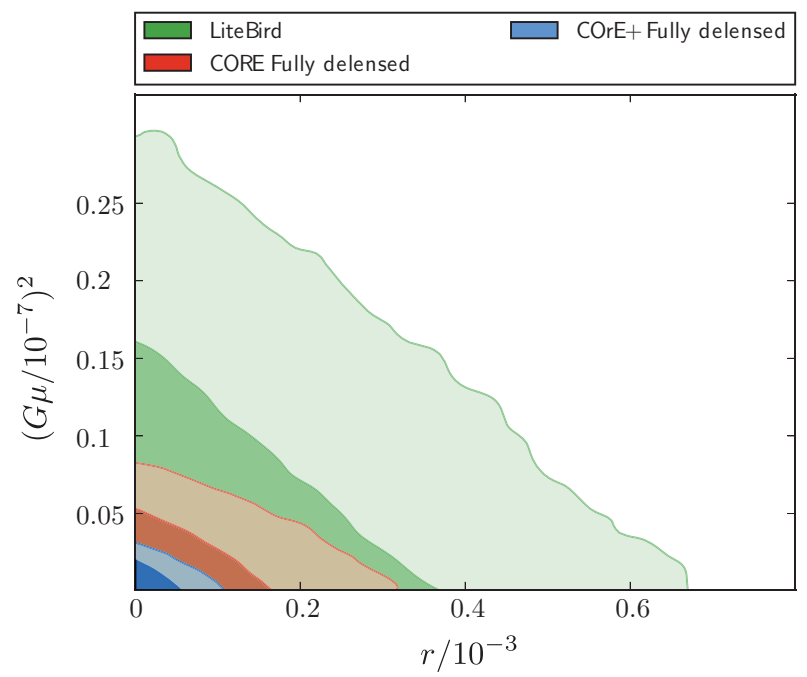

Figure 38: Likelihood contours of the $r-(G \mu)^{2}$ posterior for models with both tensors and defects (cosmic strings), marginalized over all other parameters, for LiteBIRD, CORE, and COrE + . The CORE and $\mathrm{COrE}+$ contours are given for the fully delensed case, representing the best possible constraints attainable, while LiteBIRD contours are given for the standard case. In all cases light shading represents $1 \sigma$ and dark shading $2 \sigma$. For comparison, the current Planck marginalized $95 \%$ confidence limits on these models are $\left(G \mu / 10^{-7}\right)^{2}<3.4\left(f_{10}<0.011\right)$ and $r<0.11$.

\subsection{Summary}

We find that $\mathrm{COrE}+$ places the tightest constraints on the abundance of topological defects, followed by CORE, and finally by LiteBIRD. When looking only for defects and without delensing, the limit on $f_{10}$ achievable by CORE when fitting against a strings-only model $\left(3.0 \times 10^{-4}\right)$ is approximately twice as strong as that by LiteBIRD, with COrE + about $20 \%$ stronger still.

All these future missions will perform much better than Planck, however, for which $f_{10}<0.011$ at 95\% CL [338] in models with strings and tensors. COrE + could improve the constraints on $f_{10}$ by up to two orders of magnitude, and thus by about one order of magnitude in $G \mu$.

Delensing leads to further improvements in the constraints for COrE + and CORE. If the Bmode map can be fully delensed, the improvement is a factor of 2 in $f_{10}$ for CORE and nearly a factor of 4 for COrE + . LiteBIRD will not be able to delens its data on its own.

With full delensing, the upper bound on the string tension parameter can be brought down to $G \mu<2.1 \times 10^{-8}$, which corresponds to a bound on the symmetry-breaking scale of $\phi_{0}<6.9 \times 10^{14}$, well below the GUT scale.

\section{Conclusions}

A broad consensus has emerged that the discovery of primordial gravitational waves generated during the epoch of inflation will likely be one of the next breakthrough discoveries of observational 


\begin{tabular}{llcc}
\hline B-mode analysis & \multicolumn{1}{c}{$f_{10}$} & $10^{12}(G \mu)^{2}$ & $r$ \\
\hline \hline & COrE + & & \\
\hline standard & $5.0 \times 10^{-4}$ & $1.5 \times 10^{-3}$ & $3.2 \times 10^{-4}$ \\
standard & $2.3 \times 10^{-4}$ & $7.0 \times 10^{-4}$ & - \\
fully delensed & $7.1 \times 10^{-5}$ & $2.2 \times 10^{-4}$ & $5.8 \times 10^{-5}$ \\
fully delensed & $6.3 \times 10^{-5}$ & $1.9 \times 10^{-4}$ & - \\
\hline \hline \multicolumn{4}{c}{} \\
\hline standard & CORE & \\
standard & $3.4 \times 10^{-4}$ & $1.6 \times 10^{-3}$ & $4.3 \times 10^{-4}$ \\
fully delensed & $1.9 \times 10^{-4}$ & $9.0 \times 10^{-4}$ & - \\
fully delensed & $1.5 \times 10^{-4}$ & $4.5 \times 10^{-4}$ & $1.7 \times 10^{-4}$ \\
\hline \hline & LiteBIRD & - \\
\hline standard & $6.5 \times 10^{-4}$ & $2.0 \times 10^{-3}$ & $3.3 \times 10^{-4}$ \\
standard & $7.1 \times 10^{-4}$ & $2.1 \times 10^{-3}$ & - \\
\hline
\end{tabular}

Table 26: Upper limits at 95\% confidence on the parameters in $\Lambda$ CDM models with cosmic strings and/or primordial gravitational waves tested against an input spectrum with neither strings nor gravitational waves. Note that $f_{10}$ (the proportion of the temperature power spectrum due to strings) is proportional to $(G \mu)^{2}$ where $\mu$ is the string tension and $G$ is Newton's constant.

cosmology. These would be detected through their imprint on the B mode of the CMB polarization anisotropy. The experimental requirements for a definitive search for the B mode signal from inflationary gravitational waves would lead to far reaching improvements in the measurement of $\mathrm{T}$ and $\mathrm{E}$ and would allow for a tremendous advance in our understanding of cosmic inflation, beyond measuring the energy scale of inflation from the primordial B-mode polarization amplitude.

In this paper we have explored the potential of an experiment like CORE, a proposed CMB space satellite submitted in response to the ESA fifth call for a medium-size mission opportunity, for improving our understanding of cosmic inflation. CORE would also enable a broad range of science objectives to be achieved in cosmology beyond inflation science, as reported in the companion papers of this series for cosmological parameters [9], extragalactic sources [11], clusters [12], and Doppler/aberration effects [13]. We have quantified how CORE compares to other concepts for a next generation CMB space mission dedicated to the ultimate measurement of the polarization anisotropies in improving our knowledge of the physics of inflation, at present largely based on Planck and ground experiments such as BICEP 2/Keck Array.

This paper uses idealized mock likelihoods that assume that the lowest and highest frequency channels suffice to remove foreground contaminations from the inverse noise weighted combination of the six central frequency 'cosmological' channels used for our forecasts. Although this is an idealized case, CMB space missions such as CORE (denoted in this paper as CORE-M5) are experimental concepts that come close to achieving this limit as a result of the large number of frequency channels present and the possibility to measure the Galactic dust polarization signal at high frequencies accurately. We refer the reader to the companion paper [10] for a the CORE capability to measure primordial B-mode polarization by a component separation approach on maps 
which include contributions from of all the relevant foregrounds. Table 27 summarizes the expected CORE uncertainties in the inflationary parameters compared to the current measurements based on the Planck 2015 data.

We included the JAXA LiteBIRD proposal [27, 28] among the different configurations to be compared with CORE by adopting specifications of an extended LiteBIRD mission [168]. We have shown that CORE can achieve an uncertainty in $r$ at least a factor of 2 better than the LiteBIRD configuration. Because of its coarse angular resolution (owing to its smaller aperture), LiteBIRD needs to be complemented with external CMB data to reach the level of the current Planck uncertainties for the scale dependence of the spectral index, primordial non-Gaussianities and isocurvature perturbations, which are invaluable for probing the physics of inflation beyond searching for the energy scale at which inflation occurred.

Highlights of the new information regarding the physics of inflation that can be achieved with CORE include the following:

\section{Primordial B-modes and fundamental physics}

With its sensitivity to the primordial B-mode polarization, CORE will test new physics at energy scales approximately a trillion times higher than those probed by the Large Hadron Collider (LHC). Such scales are of key relevance to fundamental physics. If gravitational waves from inflation are detected by CORE, this discovery will constitute the first experimental signature of quantum gravity and of physics at the Planck scale, implying large-field inflation with a superPlanckian field excursion. If $r$ is measured at high statistical significance, we have shown how CORE will be superior to LiteBIRD for constraining the shape of the spectrum of primordial gravitational waves. The CORE window on energy scales probed by B-mode polarization is unique. The target sensitivity of the ESA-L3 gravitational wave mission is not sufficient to detect inflationary gravitational waves for the simplest inflationary models. However, synergies between CORE and the ESA-L3 gravitational wave mission will be able to constrain alternatives to the simplest models of inflation.

\section{Boosting the precision in the measurements of cosmological parameters}

The precision of the determination of cosmological parameters will be greatly improved by CORE. We have shown that the specifications of CORE suffice to provide nearly ideal (i.e., cosmic-variance limited) measurement of the $\mathrm{CMB}$ temperature and polarization power spectra up to high multipoles. With the addition of the CMB lensing, whose information will be exploited up to the scales where linear theory is reliable, CORE will improve the uncertainties on key inflationary parameters such as $n_{\mathrm{s}}, \mathrm{d} n_{\mathrm{s}} / \mathrm{d} \ln k$, and $\Omega_{\mathrm{k}}$ by approximatively factors of $3.4,2.9$, and 4 , respectively.

\section{Slow-roll inflationary models}

CORE will explore values of $r$ approximately two orders of magnitude below the current limit. We have shown how CORE specifications are sufficient to test the predictions of the $R^{2}$ model, which is the simplest among the inflationary models favored by the Planck data. CORE could also target smaller values of $r$, as predicted for instance in maximally supersymmetric realizations 


\begin{tabular}{|c|c|c|c|}
\hline Parameter & Results from Planck 2015 release & $\begin{array}{c}\text { CORE } \\
\text { expected uncertainties }\end{array}$ & $\begin{array}{l}\text { Improvement } \\
\text { factor }\end{array}$ \\
\hline \multicolumn{4}{|l|}{$\Lambda \mathrm{CDM}$ model } \\
\hline$A_{\mathrm{s}}$ & $A_{\mathrm{s}}=(2.130 \pm 0.053) \times 10^{-9}(68 \% \mathrm{CL})[4]$ & $\sigma\left(A_{\mathrm{s}}\right)=0.0073$ & 7.3 \\
\hline$n_{\mathrm{s}}$ & $n_{\mathrm{s}}=0.9653 \pm 0.0048(68 \% \mathrm{CL})[4]$ & $\sigma\left(n_{\mathrm{s}}\right)=0.0014$ & 3.4 \\
\hline$\Omega_{\mathrm{b}} h^{2}$ & $\Omega_{\mathrm{b}} h^{2}=0.02226 \pm 0.00016(68 \% \mathrm{CL})[4]$ & $\sigma\left(\Omega_{b} h^{2}\right)=0.000037$ & 4.3 \\
\hline$\Omega_{\mathrm{c}} h^{2}$ & $\Omega_{\mathrm{c}} h^{2}=0.1193 \pm 0.0014(68 \% \mathrm{CL})[4]$ & $\sigma\left(\Omega_{c} h^{2}\right)=0.00026$ & 5.4 \\
\hline$\tau$ & $\tau=0.063 \pm 0.014(68 \% \mathrm{CL})[4]$ & $\sigma(\tau)=0.002$ & 7.0 \\
\hline$H_{0}[\mathrm{~km} / \mathrm{s} / \mathrm{Mpc}]$ & $H_{0}=67.51 \pm 0.64(68 \% \mathrm{CL})[4]$ & $\sigma\left(H_{0}\right)=0.11$ & 5.8 \\
\hline $\mathrm{d} n_{\mathrm{S}} / \mathrm{d} \ln k$ & $\mathrm{~d} n_{\mathrm{s}} / \mathrm{d} \ln k=-0.0023 \pm 0.0067(68 \% \mathrm{CL})[4,5]$ & $\sigma\left(\mathrm{d} n_{\mathrm{s}} / \mathrm{d} \ln k\right)=0.0023$ & 2.9 \\
\hline $\mathrm{d}^{2} n_{\mathrm{s}} / \mathrm{d} \ln k^{2}$ & $\mathrm{~d}^{2} n_{\mathrm{s}} / \mathrm{d} \ln k^{2}=0.025 \pm 0.013(68 \% \mathrm{CL})[5]$ & $\sigma\left(\mathrm{d}^{2} n_{\mathrm{s}} / \mathrm{d} \ln k^{2}\right)=0.0046$ & 2.8 \\
\hline$\Omega_{\mathrm{k}}$ & $\Omega_{\mathrm{k}}=-0.0037_{-0.0069}^{+0.0083}(68 \%$ CL $)[4]$ & $\sigma\left(\Omega_{\mathrm{k}}\right)=0.0019$ & 4 \\
\hline$r$ & $r<0.08(95 \% \mathrm{CL})[5,54]$ & $\begin{array}{c}\sigma(r)=4 \cdot 10^{-4} \\
\left(r_{\mathrm{fid}}=0.01\right)\end{array}$ & $10^{2}$ \\
\hline$n_{\mathrm{t}}$ & $-0.38<n_{\mathrm{t}}<2.6(95 \% \mathrm{CL})[5]$ & $\begin{array}{c}\sigma\left(n_{\mathrm{t}}\right)=0.08 \\
\left(r_{\text {fid }}=0.01, n_{\text {fid } \mathrm{t}}=-r_{\text {fid }} / 8\right)\end{array}$ & 10 \\
\hline$\beta_{\text {iso }}$ & $\begin{array}{l}\beta_{\text {iso }}^{\text {curvaton }}<0.0013(95 \% \mathrm{CL})[5] \\
\beta_{\text {iso }}^{\text {axion }}<0.038(95 \% \mathrm{CL})[5]\end{array}$ & $\begin{aligned} \beta_{\text {iso }}^{\text {curvaton }} & <0.00026(95 \% \mathrm{CL}) \\
\beta_{\text {iso }}^{\text {axion }} & <0.018(95 \% \mathrm{CL})\end{aligned}$ & $\begin{array}{l}5.0 \\
2.1\end{array}$ \\
\hline$f_{\mathrm{NL}}$ & $\begin{array}{l}f_{\mathrm{NL}}^{\text {local }}=0.8 \pm 5.0(68 \% \mathrm{CL})[52] \\
f_{\mathrm{NL}}^{\text {equil }}=-4 \pm 43(68 \% \mathrm{CL})[52] \\
f_{\mathrm{NL}}^{\mathrm{ortho}}=-26 \pm 21(68 \% \mathrm{CL})[52] \\
f_{\mathrm{NL}}^{\mathrm{ISW}-\text { lens }}=0.79 \pm 0.28(68 \% \mathrm{CL})[52]\end{array}$ & $\begin{array}{c}\sigma\left(f_{\mathrm{NL}}^{\text {local }}\right)=2.1 \\
\sigma\left(f_{\mathrm{NL}}^{\text {equil }}\right)=21 \\
\sigma\left(f_{\mathrm{NL}}^{\text {ortho }}\right)=9.6 \\
\sigma\left(f_{\mathrm{NL}}^{\mathrm{ISW}-\text { lens }}\right)=0.045\end{array}$ & $\begin{array}{l}2.4 \\
2.0 \\
2.2 \\
6.2\end{array}$ \\
\hline$c_{\mathrm{s}}$ & $c_{\mathrm{s}}>0.023(95 \% \mathrm{CL})[52]$ & $c_{\mathrm{s}}>0.045(95 \% \mathrm{CL})$ & 2.0 \\
\hline$G \mu$ & $G \mu<2.0 \times 10^{-7}(95 \%$ CL $)[338]$ & $G \mu<2.1 \times 10^{-8}(95 \% \mathrm{CL})$ & 9.5 \\
\hline
\end{tabular}

Table 27: Summary of the current results based on the latest Planck 2015 release and CORE forecasts presented in this paper. The third column gives the figure of merit of the improvement expected with CORE.

of inflation with the largest possible value of the moduli space curvature. CORE will also help provide information about the reheating stage for a given inflationary model and could allow us to distinguish models that share the same inflationary potential but have different reheating mechanisms.

\section{Testing deviations from a simple power-law}

By providing a cosmic variance limited measurement of the $E E$ power spectrum up to high multipoles, any of the proposed CORE configurations will increase the amount of information available on the scalar primordial power spectrum by an order of magnitude with respect to the existing Planck 2015 data. We have shown that if there are any features in the scalar primordial power spectrum hidden beneath Planck's resolution, CORE would reliably reconstruct them. This reconstruction can be performed in either in a model independent or a non-parametric manner, or by using more traditional parametric methods. We demonstrate this success for both low- $\ell$ and high- $\ell$ features. Additionally, CORE-like experiments would be able to establish constraints on the tensor primordial power spectra even for relatively low values of the tensor-to-scalar ratio $r$. For the purposes of primordial power spectrum reconstruction, CORE is the natural successor 
to Planck to provide answers to the many questions surrounding these critical predictions of inflationary models.

\section{Beyond the adiabatic initial condition}

CORE will determine the nature of the initial conditions of primordial fluctuations $2-5$ times better than Planck (and also LiteBIRD because of its coarse angular resolution) by providing nearly cosmic variance limited upper bounds on the allowed isocurvature fraction. By including cosmological information contained in the CMB lensing power spectrum, the isocurvature bounds will improve compared to the bounds obtained using an ideal cosmic variance limited experiment with only the temperature and polarization anisotropies. We have shown that CORE can recover $10^{-3}$ level isocurvature fractions (one order of magnitude below the current constraints) without biasing the cosmological parameters, even in the presence of tensor modes. We finally have shown that the uncertainties in the cosmological parameters of the $\Lambda \mathrm{CDM}$ model will be increased at most by $25 \%$ by allowing generally correlated isocurvature fluctuations with respect to the adiabatic case. All these analyses have been carried for a correlated mixture of adiabatic and isocurvature CDI modes, but similar results are expected for the neutrino density and velocity modes as well.

\section{Primordial non-Gaussianity}

As a figure of merit for primordial non-Gaussianities, the direct bispectrum measurements by CORE will shrink the allowed $f_{\mathrm{NL}}$ volume in the three-dimensional Local-Equilateral-Orthogonal (LEO) shape-function space by a factor of approximately 20 with respect to the current Planck results. This corresponds to signal-to-noise ratio improvements by a factor $\approx 2-3$ for each shape, giving $1 \sigma f_{\mathrm{NL}}$ error forecasts of about 2, 20, and 10 for local, equilateral, and orthogonal shapes, respectively. Similar levels of improvement are also found for other models such as oscillatory bispectra or scenarios with running of $f_{\mathrm{NL}}$, and for $g_{\mathrm{NL}}$ trispectrum constraints. Even larger improvements can be found for isocurvature NG models, where the improved polarization sensitivity is crucial. In this case $f_{\mathrm{NL}}$ error bars shrink by a factor of up to $\sim 10$.

Very interesting results are also expected if we consider alternative ways of estimating local NG not based on direct temperature and polarization bispectrum estimation. Estimating the power spectrum of the correlation between temperature and $\mu$-distortion anisotropies can for example lead to better $f_{\mathrm{NL}}$ constraints by a factor of up to $\sim 100$ for specific models with excited initial states. Finally, a crucial finding is that through scale-dependent bias measurements of the CIB power spectrum on large scales, CORE will be able to achieve $\sigma\left(f_{\mathrm{NL}}\right) \lesssim 1$ for the local shape. This is a crucial threshold for discriminating between single- and multi-field inflation, and such a level of sensitivity would be comparable to or better than the sensitivity expected from future galaxy surveys.

\section{Topological Defects}

CORE will search for primordial B-modes generated by vector and tensor perturbations induced by topological defects, including cosmic strings. The resulting B-mode power spectrum produced by topological defects is quite different from the shape produced by inflationary gravitational waves. CORE improves the limits from the CMB anisotropy power spectrum on Abelian 
Higgs gauge cosmic strings by nearly an order of magnitude compared to Planck, pushing the maximally allowed string tension $G \mu$ decisively below the GUT scale (or detecting cosmic strings if any are formed at GUT energy scales). Similar gains in sensitivity are expected for all types of defects. Previous work [331] indicates that a mission with CORE's capabilities can not only detect topological defects, but also distinguish them from primordial tensors and even from each other. CORE can therefore probe different physical mechanisms generating a B-mode signal at the GUT energy scale.

\section{Acknowledgements}

FA is supported by the National Taiwan University (NTU) under Project No. $103 \mathrm{R} 4000$ and by the NTU Leung Center for Cosmology and Particle Astrophysics (LeCosPA) under Project No. FI121. KK acknowledges support by the Magnus Ehrnrooth Foundation. JV acknowledges support by the Finnish Cultural Foundation. KK and JV work is supported in part by Academy of Finland grants 257989 and 295113. CJM is supported by an FCT Research Professorship, contract reference IF/00064/2012, funded by FCT/MCTES (Portugal) and POPH/FSE (EC). Partial support by ASI/INAF Agreement 2014-024-R.1 for the Planck LFI Activity of Phase E2. The calculations in this work were performed in CSC - the IT Center for Science Ltd. (Finland), IN2P3 Computer Center (France), HPC-cluster facilities of RWTH Aachen University (Germany), INAF-IASF Bologna HPC cluster (Italy), the Cambridge COSMOS SMP system (UK), part of the STFC DiRAC HPC Facility supported by BIS NeI capital grant ST/J005673/1 and STFC grants ST/H008586/1, ST/K00333X/1.

\section{References}

[1] G. F. Smoot et al., Structure in the COBE differential microwave radiometer first year maps, Astrophys. J. 396 (1992) L1.

[2] Planck collaboration, P. A. R. Ade et al., Planck 2013 results. XVI. Cosmological parameters, Astron. Astrophys. 571 (2014) A16, [1303.5076].

[3] Planck collaboration, P. A. R. Ade et al., Planck 2013 results. XXII. Constraints on inflation, Astron. Astrophys. 571 (2014) A22, [1303.5082].

[4] Planck collaboration, P. A. R. Ade et al., Planck 2015 results. XIII. Cosmological parameters, Astron. Astrophys. 594 (2016) A13, [1502.01589].

[5] Planck collaboration, P. A. R. Ade et al., Planck 2015 results. XX. Constraints on inflation, Astron. Astrophys. 594 (2016) A20, [1502.02114].

[6] CORE Collaboration, Exploring Cosmic Origins with CORE: The CORE mission, In preparation .

[7] CORE Collaboration, Exploring Cosmic Origins with CORE: the Instrument, In preparation .

[8] CORE Collaboration, Exploring Cosmic Origins with CORE: Mitigation of Systematic effects, In preparation .

[9] CORE collaboration, E. Di Valentino et al., Exploring Cosmic Origins with CORE: Cosmological Parameters, 1612.00021. 
[10] CORE Collaboration, Exploring Cosmic Origins with CORE: B-mode component separation, In preparation .

[11] CORE collaboration, G. De Zotti et al., Exploring Cosmic Origins with CORE: Extragalactic sources in Cosmic Microwave Background maps, 1609.07263.

[12] CORE Collaboration, Exploring Cosmic Origins with CORE: Cluster Science, In preparation .

[13] CORE Collaboration, Exploring Cosmic Origins with CORE: effects of observer peculiar motion, In preparation .

[14] B-Pol collaboration, P. de Bernardis, M. Bucher, C. Burigana and L. Piccirillo, B-Pol: Detecting Primordial Gravitational Waves Generated During Inflation, Exper. Astron. 23 (2009) 5-16, [0808.1881].

[15] COrE collaboration, F. R. Bouchet et al., COrE (Cosmic Origins Explorer) A White Paper, 1102.2181.

[16] PRISM collaboration, P. André et al., PRISM (Polarized Radiation Imaging and Spectroscopy Mission): An Extended White Paper, JCAP 1402 (2014) 006, [1310.1554].

[17] CMBPol Study Team collaboration, D. Baumann et al., CMBPol Mission Concept Study: Probing Inflation with CMB Polarization, AIP Conf. Proc. 1141 (2009) 10-120, [0811.3919].

[18] J. Dunkley et al., CMBPol Mission Concept Study: Prospects for polarized foreground removal, AIP Conf. Proc. 1141 (2009) 222, [0811.3915].

[19] A. A. Fraisse et al., CMBPol Mission Concept Study: Foreground Science Knowledge and Prospects, AIP Conf. Proc. 1141 (2009) 265, [0811.3920].

[20] K. M. Smith et al., CMBPol Mission Concept Study: Gravitational Lensing, AIP Conf. Proc. 1141 (2009) 121, [0811.3916].

[21] CMBPol Study Team collaboration, D. Baumann, A. Cooray, S. Dodelson, J. Dunkley, A. A. Fraisse, M. G. Jackson et al., CMBPol Mission Concept Study: A Mission to Map our Origins, AIP Conf. Proc. 1141 (2009) 3-9, [0811.3911].

[22] EPIC collaboration, J. Bock et al., Study of the Experimental Probe of Inflationary Cosmology (EPIC)-Intemediate Mission for NASA's Einstein Inflation Probe, 0906.1188.

[23] J. Bock, A. Cooray, S. Hanany, B. Keating, A. Lee, T. Matsumura et al., The Experimental Probe of Inflationary Cosmology (EPIC): A Mission Concept Study for NASA's Einstein Inflation Probe, 0805.4207.

[24] A. Kogut et al., The Primordial Inflation Explorer (PIXIE): A Nulling Polarimeter for Cosmic Microwave Background Observations, JCAP 1107 (2011) 025, [1105.2044].

[25] D. J. Fixsen, E. S. Cheng, J. M. Gales, J. C. Mather, R. A. Shafer and E. L. Wright, The Cosmic Microwave Background spectrum from the full COBE FIRAS data set, Astrophys. J. 473 (1996) 576, [astro-ph/9605054].

[26] A. Kogut, J. Chluba, D. J. Fixsen, S. Meyer and D. Spergel, The Primordial Inflation Explorer (PIXIE), in Society of Photo-Optical Instrumentation Engineers (SPIE) Conference Series, vol. 9904 of SPIE, p. $99040 \mathrm{~W}, 2016$. DOI.

[27] T. Matsumura, Y. Akiba, J. Borrill, Y. Chinone, M. Dobbs, H. Fuke et al., Mission Design of LiteBIRD, J. Low Temp. Phys. 176 (2014) 733, [1311.2847].

[28] T. Matsumura et al., LiteBIRD: Mission Overview and Focal Plane Layout, J. Low. Temp. Phys. 184 (2016) 824-831. 
[29] R. Brout, F. Englert and E. Gunzig, The Creation of the Universe as a Quantum Phenomenon, Annals Phys. 115 (1978) 78.

[30] A. A. Starobinsky, A New Type of Isotropic Cosmological Models Without Singularity, Phys. Lett. B91 (1980) 99.

[31] D. Kazanas, Dynamics of the Universe and Spontaneous Symmetry Breaking, Astrophys. J. 241 (1980) L59-L63.

[32] K. Sato, First Order Phase Transition of a Vacuum and Expansion of the Universe, Mon.Not.Roy.Astron.Soc. 195 (1981) 467.

[33] A. H. Guth, The Inflationary Universe: A Possible Solution to the Horizon and Flatness Problems, Phys.Rev. D23 (1981) 347.

[34] A. D. Linde, A New Inflationary Universe Scenario: A Possible Solution of the Horizon, Flatness, Homogeneity, Isotropy and Primordial Monopole Problems, Phys.Lett. B108 (1982) 389.

[35] A. D. Linde, Chaotic Inflation, Phys.Lett. B129 (1983) 177.

[36] A. Albrecht and P. J. Steinhardt, Cosmology for Grand Unified Theories with Radiatively Induced Symmetry Breaking, Phys.Rev.Lett. 48 (1982) 1220.

[37] Boomerang collaboration, P. de Bernardis et al., A Flat universe from high resolution maps of the cosmic microwave background radiation, Nature 404 (2000) 955-959, [astro-ph/0004404].

[38] S. Hanany et al., MAXIMA-1: A Measurement of the cosmic microwave background anisotropy on angular scales of 10 arcminutes to 5 degrees, Astrophys. J. 545 (2000) L5, [astro-ph/0005123].

[39] WMAP collaboration, D. N. Spergel et al., First year Wilkinson Microwave Anisotropy Probe (WMAP) observations: Determination of cosmological parameters, Astrophys. J. Suppl. 148 (2003) 175-194, [astro-ph/0302209].

[40] J. Dunkley et al., The Atacama Cosmology Telescope: Cosmological Parameters from the 2008 Power Spectra, Astrophys. J. 739 (2011) 52, [1009.0866].

[41] R. Keisler et al., A Measurement of the Damping Tail of the Cosmic Microwave Background Power Spectrum with the South Pole Telescope, Astrophys. J. 743 (2011) 28, [1105.3182].

[42] SDSS collaboration, D. J. Eisenstein et al., Detection of the baryon acoustic peak in the large-scale correlation function of SDSS luminous red galaxies, Astrophys. J. 633 (2005) 560-574, [astro-ph/0501171].

[43] SDSS collaboration, W. J. Percival et al., Baryon Acoustic Oscillations in the Sloan Digital Sky Survey Data Release 7 Galaxy Sample, Mon. Not. Roy. Astron. Soc. 401 (2010) 2148-2168, [0907.1660].

[44] Planck collaboration, P. A. R. Ade et al., Planck 2013 results. XVII. Gravitational lensing by large-scale structure, Astron. Astrophys. 571 (2014) A17, [1303.5077].

[45] Planck collaboration, P. A. R. Ade et al., Planck 2015 results. XV. Gravitational lensing, Astron. Astrophys. 594 (2016) A15, [1502.01591].

[46] A. G. Riess et al., A 2.4\% Determination of the Local Value of the Hubble Constant, Astrophys. J. 826 (2016) 56, [1604.01424].

[47] Planck collaboration, P. A. R. Ade et al., Planck 2013 results. XX. Cosmology from Sunyaev-Zeldovich cluster counts, Astron. Astrophys. 571 (2014) A20, [1303.5080]. 
[48] Planck collaboration, P. A. R. Ade et al., Planck 2015 results. XXIV. Cosmology from Sunyaev-Zeldovich cluster counts, Astron. Astrophys. 594 (2016) A24, [1502.01597].

[49] S. Joudaki et al., CFHTLenS revisited: Assessing concordance with Planck including astrophysical systematics, 1601.05786.

[50] H. Hildebrandt et al., KiDS-450: Cosmological parameter constraints from tomographic weak gravitational lensing, 1606.05338.

[51] Planck collaboration, P. A. R. Ade et al., Planck 2013 Results. XXIV. Constraints on primordial non-Gaussianity, Astron. Astrophys. 571 (2014) A24, [1303.5084].

[52] Planck collaboration, P. A. R. Ade et al., Planck 2015 results. XVII. Constraints on primordial non-Gaussianity, Astron. Astrophys. 594 (2016) A17, [1502.01592].

[53] WMAP collaboration, C. L. Bennett et al., Nine-Year Wilkinson Microwave Anisotropy Probe (WMAP) Observations: Final Maps and Results, Astrophys. J. Suppl. 208 (2013) 20, [1212.5225].

[54] BICEP2, Planck collaboration, P. A. R. Ade et al., Joint Analysis of BICEP2/Keck?Array and Planck Data, Phys. Rev. Lett. 114 (2015) 101301, [1502.00612].

[55] BICEP2, Keck Array collaboration, P. A. R. Ade et al., Improved Constraints on Cosmology and Foregrounds from BICEP2 and Keck Array Cosmic Microwave Background Data with Inclusion of 95 GHz Band, Phys. Rev. Lett. 116 (2016) 031302, [1510.09217].

[56] L. Knox, Determination of inflationary observables by cosmic microwave background anisotropy experiments, Phys. Rev. D52 (1995) 4307, [astro-ph/9504054].

[57] H.-Y. Wu and O. Doré, Optimizing future experiments of cosmic far-infrared background: a principal component approach, 1612.02474.

[58] M. Bucher, B. Van Tent and C. S. Carvalho, Detecting Bispectral Acoustic Oscillations from Inflation Using a New Flexible Estimator, Mon. Not. Roy. Astron. Soc. 407 (2010) 2193, [0911.1642].

[59] BICEP3 collaboration, J. A. Grayson et al., BICEP3 performance overview and planned Keck Array upgrade, Proc. SPIE Int. Soc. Opt. Eng. 9914 (2016) 99140S, [1607.04668].

[60] A. A. Fraisse et al., SPIDER: Probing the Early Universe with a Suborbital Polarimeter, JCAP 1304 (2013) 047, [1106.3087].

[61] POLARBEAR collaboration, A. Suzuki et al., The POLARBEAR-2 and the Simons Array Experiment, J. Low. Temp. Phys. 184 (2016) 805, [1512.07299].

[62] S. W. Henderson et al., Advanced ACTPol Cryogenic Detector Arrays and Readout, J. Low. Temp. Phys. 184 (2016) 772, [1510.02809].

[63] SPT-3G collaboration, B. A. Benson et al., SPT-3G: A Next-Generation Cosmic Microwave Background Polarization Experiment on the South Pole Telescope, Proc. SPIE Int. Soc. Opt. Eng. 9153 (2014) 91531P, [1407.2973].

[64] PIPER collaboration, N. N. Gandilo et al., The Primordial Inflation Polarization Explorer (PIPER), Proc. SPIE Int. Soc. Opt. Eng. 9914 (2016) 99141J, [1607.06172].

[65] K. Harrington et al., The Cosmology Large Angular Scale Surveyor, Proc. SPIE Int. Soc. Opt. Eng. 9914 (2016) 99141K, [1608.08234].

[66] LSPE collaboration, S. Aiola et al., The Large-Scale Polarization Explorer (LSPE), Proc. SPIE Int. Soc. Opt. Eng. 8446 (2012) 84467A, [1208.0281]. 
[67] J. A. Rubiño-Martín, R. Rebolo, M. Aguiar, R. Génova-Santos, F. Gómez-Reñasco, J. M. Herreros et al., The QUIJOTE-CMB experiment: studying the polarisation of the galactic and cosmological microwave emissions, in Society of Photo-Optical Instrumentation Engineers (SPIE) Conference Series, vol. 8444, Sept., 2012. DOI.

[68] CMB-S4 collaboration, K. N. Abazajian et al., CMB-S4 Science Book, First Edition, 1610.02743.

[69] BICEP2 collaboration, P. A. R. Ade et al., Detection of B-Mode Polarization at Degree Angular Scales by BICEP2, Phys. Rev. Lett. 112 (2014) 241101, [1403.3985].

[70] Planck collaboration, P. A. R. Ade et al., Planck 2013 results. XV. CMB power spectra and likelihood, Astron. Astrophys. 571 (2014) A15, [1303.5075].

[71] Planck collaboration, N. Aghanim et al., Planck 2015 results. XI. CMB power spectra, likelihoods, and robustness of parameters, Submitted to: Astron. Astrophys. (2015) , [1507.02704].

[72] Planck collaboration, N. Aghanim et al., Planck intermediate results. XLVI. Reduction of large-scale systematic effects in HFI polarization maps and estimation of the reionization optical depth, Astron. Astrophys. 596 (2016) A107, [1605.02985].

[73] Planck collaboration, R. Adam et al., Planck intermediate results. XLVII. Planck constraints on reionization history, Astron. Astrophys. 596 (2016) A108, [1605.03507].

[74] E. Silverstein and A. Westphal, Monodromy in the CMB: Gravity Waves and String Inflation, Phys. Rev. D78 (2008) 106003, [0803.3085].

[75] L. McAllister, E. Silverstein and A. Westphal, Gravity Waves and Linear Inflation from Axion Monodromy, Phys. Rev. D82 (2010) 046003, [0808.0706].

[76] F. Marchesano, G. Shiu and A. M. Uranga, F-term Axion Monodromy Inflation, JHEP 09 (2014) $184,[1404.3040]$.

[77] S. Dimopoulos, S. Kachru, J. McGreevy and J. G. Wacker, N-flation, JCAP 0808 (2008) 003, [hep-th/0507205].

[78] J. Ellis, D. V. Nanopoulos and K. A. Olive, No-Scale Supergravity Realization of the Starobinsky Model of Inflation, Phys. Rev. Lett. 111 (2013) 111301, [1305.1247].

[79] R. Kallosh and A. Linde, Superconformal generalizations of the Starobinsky model, JCAP 1306 (2013) 028, [1306.3214].

[80] W. Buchmuller, V. Domcke and K. Kamada, The Starobinsky Model from Superconformal D-Term Inflation, Phys. Lett. B726 (2013) 467, [1306.3471].

[81] F. Farakos, A. Kehagias and A. Riotto, On the Starobinsky Model of Inflation from Supergravity, Nucl. Phys. B876 (2013) 187, [1307.1137].

[82] S. Ferrara, R. Kallosh and A. Van Proeyen, On the Supersymmetric Completion of $R+R^{2}$ Gravity and Cosmology, JHEP 11 (2013) 134, [1309.4052].

[83] L. Covi, M. Gomez-Reino, C. Gross, J. Louis, G. A. Palma and C. A. Scrucca, de Sitter vacua in no-scale supergravities and Calabi-Yau string models, JHEP 06 (2008) 057, [0804.1073].

[84] L. Covi, M. Gomez-Reino, C. Gross, J. Louis, G. A. Palma and C. A. Scrucca, Constraints on modular inflation in supergravity and string theory, JHEP 08 (2008) 055, [0805.3290].

[85] R. Kallosh, A. Linde and D. Roest, Superconformal Inflationary $\alpha$-Attractors, JHEP 11 (2013) 198, [1311.0472]. 
[86] A. Hetz and G. A. Palma, Sound Speed of Primordial Fluctuations in Supergravity Inflation, Phys. Rev. Lett. 117 (2016) 101301, [1601.05457].

[87] S. Ferrara, R. Kallosh and A. Linde, Cosmology with Nilpotent Superfields, JHEP 10 (2014) 143, [1408.4096].

[88] R. Allahverdi, K. Enqvist, J. Garcia-Bellido and A. Mazumdar, Gauge invariant MSSM inflaton, Phys. Rev. Lett. 97 (2006) 191304, [hep-ph/0605035].

[89] R. Allahverdi, K. Enqvist, J. Garcia-Bellido, A. Jokinen and A. Mazumdar, MSSM flat direction inflation: Slow roll, stability, fine tunning and reheating, JCAP 0706 (2007) 019, [hep-ph/0610134].

[90] D. Baumann and D. Green, Signatures of Supersymmetry from the Early Universe, Phys. Rev. D85 (2012) 103520, [1109.0292].

[91] L. V. Delacretaz, V. Gorbenko and L. Senatore, The Supersymmetric Effective Field Theory of Inflation, 1610.04227.

[92] F. L. Bezrukov and M. Shaposhnikov, The Standard Model Higgs boson as the inflaton, Phys. Lett. B659 (2008) 703, [0710.3755].

[93] A. De Simone, M. P. Hertzberg and F. Wilczek, Running Inflation in the Standard Model, Phys. Lett. B678 (2009) 1, [0812.4946].

[94] C. Cheung, P. Creminelli, A. L. Fitzpatrick, J. Kaplan and L. Senatore, The Effective Field Theory of Inflation, JHEP 03 (2008) 014, [0709.0293].

[95] D. H. Lyth, What would we learn by detecting a gravitational wave signal in the cosmic microwave background anisotropy?, Phys. Rev. Lett. 78 (1997) 1861.

[96] D. Baumann and L. McAllister, A Microscopic Limit on Gravitational Waves from D-brane Inflation, Phys. Rev. D75 (2007) 123508, [hep-th/0610285].

[97] V. Mukhanov, Quantum Cosmological Perturbations: Predictions and Observations, Eur. Phys. J. C73 (2013) 2486, [1303.3925].

[98] D. Roest, Universality classes of inflation, JCAP 1401 (2014) 007, [1309.1285].

[99] J. Garcia-Bellido and D. Roest, Large- $N$ running of the spectral index of inflation, Phys. Rev. D89 (2014) 103527, [1402.2059].

[100] J. Garcia-Bellido, D. Roest, M. Scalisi and I. Zavala, Lyth bound of inflation with a tilt, Phys. Rev. D90 (2014) 123539, [1408.6839].

[101] P. Creminelli, S. Dubovsky, D. López Nacir, M. Simonović, G. Trevisan, G. Villadoro et al., Implications of the scalar tilt for the tensor-to-scalar ratio, Phys. Rev. D92 (2015) 123528, [1412.0678].

[102] J. Martin, C. Ringeval and V. Vennin, Encyclopaedia Inflationaris, Phys. Dark Univ. 5-6 (2014) $75,[1303.3787]$.

[103] D. Baumann, H. Lee and G. L. Pimentel, High-Scale Inflation and the Tensor Tilt, JHEP 01 (2016) 101, [1507.07250].

[104] P. Creminelli, J. Gleyzes, J. Noreña and F. Vernizzi, Resilience of the standard predictions for primordial tensor modes, Phys. Rev. Lett. 113 (2014) 231301, [1407.8439].

[105] P. Creminelli, M. A. Luty, A. Nicolis and L. Senatore, Starting the Universe: Stable Violation of 
the Null Energy Condition and Non-standard Cosmologies, JHEP 12 (2006) 080, [hep-th/0606090].

[106] Y. Cai, Y.-T. Wang and Y.-S. Piao, Is there an effect of a nontrivial $c_{T}$ during inflation?, Phys. Rev. D93 (2016) 063005, [1510.08716].

[107] Y. Cai, Y.-T. Wang and Y.-S. Piao, Propagating speed of primordial gravitational waves and inflation, Phys. Rev. D94 (2016) 043002, [1602.05431].

[108] S. Endlich, A. Nicolis and J. Wang, Solid Inflation, JCAP 1310 (2013) 011, [1210.0569].

[109] N. Bartolo, D. Cannone, A. Ricciardone and G. Tasinato, Distinctive Signatures of Spacetime Diffeomorphism Breaking in EFT of Inflation, JCAP 1603 (2016) 044, [1511.07414].

[110] D. Cannone, G. Tasinato and D. Wands, Generalised Tensor Fluctuations and Inflation, JCAP 1501 (2015) 029, [1409.6568].

[111] S. Dubovsky, R. Flauger, A. Starobinsky and I. Tkachev, Signatures of a Graviton Mass in the Cosmic Microwave Background, Phys. Rev. D81 (2010) 023523, [0907.1658].

[112] Virgo, LigO Scientific collaboration, B. P. Abbott et al., Tests of general relativity with GW150914, 1602.03841.

[113] L. Senatore, E. Silverstein and M. Zaldarriaga, New Sources of Gravitational Waves during Inflation, JCAP 1408 (2014) 016, [1109.0542].

[114] M. Mirbabayi, L. Senatore, E. Silverstein and M. Zaldarriaga, Gravitational Waves and the Scale of Inflation, Phys. Rev. D91 (2015) 063518, [1412.0665].

[115] J. Cook and L. Sorbo, Particle Production during Inflation and Gravitational Waves Detectable by Ground-Based Interferometers, Phys. Rev. D85 (2012) 023534, [1109.0022].

[116] O. Özsoy, K. Sinha and S. Watson, How Well Can We Really Determine the Scale of Inflation?, Phys. Rev. D91 (2015) 103509, [1410.0016].

[117] J. M. Maldacena, Non-Gaussian features of primordial fluctuations in single field inflationary models, JHEP 05 (2003) 013, [astro-ph/0210603].

[118] P. D. Meerburg, J. Meyers, A. van Engelen and Y. Ali-Haïmoud, On CMB B-Mode Non-Gaussianity, 1603.02243.

[119] N. Arkani-Hamed and J. Maldacena, Cosmological Collider Physics, 1503.08043.

[120] H. Lee, D. Baumann and G. L. Pimentel, Non-Gaussianity as a Particle Detector, 1607.03735.

[121] L. Bordin, P. Creminelli, M. Mirbabayi and J. Noreña, Tensor Squeezed Limits and the Higuchi Bound, 1605.08424.

[122] A. Gruzinov, Elastic inflation, Phys. Rev. D70 (2004) 063518, [astro-ph/0404548].

[123] A. Maleknejad and M. M. Sheikh-Jabbari, Gauge-flation: Inflation From Non-Abelian Gauge Fields, Phys. Lett. B723 (2013) 224, [1102.1513].

[124] P. Adshead and M. Wyman, Chromo-Natural Inflation: Natural Inflation on a Steep Potential with Classical Non-Abelian Gauge Fields, Phys. Rev. Lett. 108 (2012) 261302, [1202. 2366].

[125] M. Akhshik, Clustering Fossils in Solid Inflation, JCAP 1505 (2015) 043, [1409.3004].

[126] G. Cabass, A. Melchiorri and E. Pajer, $\mu$ distortions or running: A guaranteed discovery from CMB spectrometry, Phys. Rev. D93 (2016) 083515, [1602.05578]. 
[127] M. Alishahiha, E. Silverstein and D. Tong, DBI in the Sky, Phys. Rev. D70 (2004) 123505, [hep-th/0404084].

[128] X. Chen, M.-x. Huang, S. Kachru and G. Shiu, Observational signatures and non-Gaussianities of general single field inflation, JCAP 0701 (2007) 002, [hep-th/0605045].

[129] D. Baumann, D. Green, H. Lee and R. A. Porto, Signs of Analyticity in Single-Field Inflation, Phys. Rev. D93 (2016) 023523, [1502.07304].

[130] D. Baumann, D. Green and R. A. Porto, B-modes and the Nature of Inflation, JCAP 1501 (2015) 016, [1407.2621].

[131] A. Achúcarro, V. Atal and Y. Welling, On the viability of $m^{2} \phi^{2}$ and natural inflation, JCAP 1507 (2015) 008, [1503.07486].

[132] K. Enqvist and M. S. Sloth, Adiabatic CMB perturbations in pre - big bang string cosmology, Nucl.Phys. B626 (2002) 395, [hep-ph/0109214].

[133] D. H. Lyth and D. Wands, Generating the curvature perturbation without an inflaton, Phys.Lett. B524 (2002) 5, [hep-ph/0110002].

[134] T. Moroi and T. Takahashi, Effects of cosmological moduli fields on cosmic microwave background, Phys. Lett. B522 (2001) 215, [hep-ph/0110096].

[135] X. Chen and Y. Wang, Quasi-Single Field Inflation and Non-Gaussianities, JCAP 1004 (2010) 027, [0911.3380].

[136] T. Noumi, M. Yamaguchi and D. Yokoyama, Effective field theory approach to quasi-single field inflation and effects of heavy fields, JHEP 06 (2013) 051, [1211.1624].

[137] T. Banks and L. Mannelli, De Sitter vacua, renormalization and locality, Phys. Rev. D67 (2003) 065009, [hep-th/0209113].

[138] N. Kaloper, M. Kleban, A. Lawrence, S. Shenker and L. Susskind, Initial conditions for inflation, JHEP 11 (2002) 037, [hep-th/0209231].

[139] R. Holman and A. J. Tolley, Enhanced Non-Gaussianity from Excited Initial States, JCAP 0805 (2008) 001, [0710.1302].

[140] N. Agarwal, R. Holman, A. J. Tolley and J. Lin, Effective field theory and non-Gaussianity from general inflationary states, JHEP 05 (2013) 085, [1212.1172].

[141] J. Chluba, J. Hamann and S. P. Patil, Features and New Physical Scales in Primordial Observables: Theory and Observation, Int. J. Mod. Phys. D24 (2015) 1530023, [1505.01834].

[142] A. Achucarro, J.-O. Gong, S. Hardeman, G. A. Palma and S. P. Patil, Features of heavy physics in the CMB power spectrum, JCAP 1101 (2011) 030, [1010.3693].

[143] R. Flauger, M. Mirbabayi, L. Senatore and E. Silverstein, Productive Interactions: heavy particles and non-Gaussianity, 1606.00513.

[144] X. Chen, R. Easther and E. A. Lim, Large Non-Gaussianities in Single Field Inflation, JCAP 0706 (2007) 023, [astro-ph/0611645].

[145] A. Achúcarro, J.-O. Gong, G. A. Palma and S. P. Patil, Correlating features in the primordial spectra, Phys. Rev. D87 (2013) 121301, [1211.5619].

[146] S. Mooij, G. A. Palma, G. Panotopoulos and A. Soto, Consistency relations for sharp inflationary non-Gaussian features, JCAP 1609 (2016) 004, [1604.03533]. 
[147] P. D. Meerburg, M. Münchmeyer and B. Wandelt, Joint resonant CMB power spectrum and bispectrum estimation, Phys. Rev. D93 (2016) 043536, [1510.01756].

[148] X. Chen, R. Easther and E. A. Lim, Generation and Characterization of Large Non-Gaussianities in Single Field Inflation, JCAP 0804 (2008) 010, [0801.3295].

[149] R. Flauger and E. Pajer, Resonant Non-Gaussianity, JCAP 1101 (2011) 017, [1002.0833].

[150] S. Hotchkiss and S. Sarkar, Non-Gaussianity from violation of slow-roll in multiple inflation, JCAP 1005 (2010) 024, [0910.3373].

[151] A. Achucarro, V. Atal, B. Hu, P. Ortiz and J. Torrado, Inflation with moderately sharp features in the speed of sound: Generalized slow roll and in-in formalism for power spectrum and bispectrum, Phys. Rev. D90 (2014) 023511, [1404.7522].

[152] M. Bucher, K. Moodley and N. Turok, The General primordial cosmic perturbation, Phys. Rev. D62 (2000) 083508, [astro-ph/9904231].

[153] M. Bucher, K. Moodley and N. Turok, Constraining isocurvature perturbations with CMB polarization, Phys. Rev. Lett. 87 (2001) 191301, [astro-ph/0012141].

[154] S. Mollerach, Isocurvature Baryon Perturbations and Inflation, Phys. Rev. D42 (1990) 313.

[155] S. Weinberg, Must cosmological perturbations remain non-adiabatic after multi-field inflation?, Phys. Rev. D70 (2004) 083522, [astro-ph/0405397].

[156] M. Beltran, J. Garcia-Bellido, J. Lesgourgues and M. Viel, Squeezing the window on isocurvature modes with the lyman-alpha forest, Phys. Rev. D72 (2005) 103515, [astro-ph/0509209].

[157] S. R. Coleman and F. De Luccia, Gravitational Effects on and of Vacuum Decay, Phys. Rev. D21 (1980) 3305 .

[158] T. Kibble, Topology of Cosmic Domains and Strings, J.Phys. A9 (1976) 1387.

[159] A. D. Linde, Hybrid inflation, Phys.Rev. D49 (1994) 748, [astro-ph/9307002].

[160] E. J. Copeland, A. R. Liddle, D. H. Lyth, E. D. Stewart and D. Wands, False vacuum inflation with Einstein gravity, Phys.Rev. D49 (1994) 6410, [astro-ph/9401011].

[161] K. Kamada, Y. Miyamoto, D. Yamauchi and J. Yokoyama, Effects of cosmic strings with delayed scaling on CMB anisotropy, 1407.2951.

[162] C. Ringeval, D. Yamauchi, J. Yokoyama and F. R. Bouchet, Large scale CMB anomalies from thawing cosmic strings, JCAP 1602 (2016) 033, [1510.01916].

[163] A. Rajantie, Formation of topological defects in gauge field theories, Int.J.Mod.Phys. A17 (2002) 1, [hep-ph/0108159].

[164] E. J. Copeland, L. Pogosian and T. Vachaspati, Seeking String Theory in the Cosmos, Class.Quant.Grav. 28 (2011) 204009, [1105.0207].

[165] M. Hindmarsh, Signals of Inflationary Models with Cosmic Strings, Prog.Theor.Phys.Suppl. 190 (2011) 197, [1106.0391].

[166] R. Battye, B. Garbrecht and A. Moss, Tight constraints on F-and D-term hybrid inflation scenarios, Phys.Rev. D81 (2010) 123512, [1001.0769].

[167] J. Urrestilla, P. Mukherjee, A. R. Liddle, N. Bevis, M. Hindmarsh et al., Degeneracy between primordial tensor modes and cosmic strings in future CMB data from the Planck satellite, Phys.Rev. D77 (2008) 123005, [0803.2059]. 
[168] J. Errard, S. M. Feeney, H. V. Peiris and A. H. Jaffe, Robust forecasts on fundamental physics from the foreground-obscured, gravitationally-lensed CMB polarization, JCAP 1603 (2016) 052, [1509.06770].

[169] L. Verde, H. Peiris and R. Jimenez, Optimizing CMB polarization experiments to constrain inflationary physics, JCAP 0601 (2006) 019, [astro-ph/0506036].

[170] P. Creminelli, D. L. López Nacir, M. Simonović, G. Trevisan and M. Zaldarriaga, Detecting Primordial B-Modes after Planck, JCAP 1511 (2015) 031, [1502.01983].

[171] M. Kamionkowski and E. D. Kovetz, The Quest for B Modes from Inflationary Gravitational Waves, 1510.06042.

[172] BICEP2 Collaboration, Keck Array Collaboration, P. A. R. Ade, Z. Ahmed, R. W. Aikin, K. D. Alexander et al., Improved Constraints on Cosmology and Foregrounds from BICEP2 and Keck Array Cosmic Microwave Background Data with Inclusion of 95 GHz Band, Phys. Rev. Lett. 116 (2016) 031302, [1510.09217].

[173] S. Hamimeche and A. Lewis, Likelihood Analysis of CMB Temperature and Polarization Power Spectra, Phys. Rev. D77 (2008) 103013, [0801.0554].

[174] M. Kaplinghat, L. Knox and Y.-S. Song, Determining Neutrino Mass from the Cosmic Microwave Background Alone, Phys. Rev. Lett. 91 (2003) 241301, [astro-ph/0303344].

[175] A. Lewis, A. Challinor and A. Lasenby, Efficient computation of CMB anisotropies in closed FRW models, Astrophys. J. 538 (2000) 473, [astro-ph/9911177].

[176] J. Chluba and R. M. Thomas, Towards a complete treatment of the cosmological recombination problem, Mon. Not. Roy. Astron. Soc. 412 (2011) 748, [1010.3631].

[177] Y. Ali-Haimoud and C. M. Hirata, HyRec: A fast and highly accurate primordial hydrogen and helium recombination code, Phys. Rev. D83 (2011) 043513, [1011.3758].

[178] P. Collaboration, Planck intermediate results. XLVI. Reduction of large-scale systematic effects in HFI polarization maps and estimation of the reionization optical depth, Astronomy $\mathbb{E}$ Astrophysics, in press (2016), [1605.02985].

[179] A. Lewis and S. Bridle, Cosmological parameters from CMB and other data: A Monte Carlo approach, Phys. Rev. D66 (2002) 103511, [astro-ph/0205436].

[180] F. Feroz and M. P. Hobson, Multimodal nested sampling: an efficient and robust alternative to MCMC methods for astronomical data analysis, Mon. Not. Roy. Astron. Soc. 384 (2008) 449, [0704.3704].

[181] F. Feroz, M. P. Hobson and M. Bridges, MultiNest: an efficient and robust Bayesian inference tool for cosmology and particle physics, Mon. Not. Roy. Astron. Soc. 398 (2009) 1601, [0809.3437].

[182] F. Feroz, M. P. Hobson, E. Cameron and A. N. Pettitt, Importance Nested Sampling and the MultiNest Algorithm, 1306.2144.

[183] W. J. Handley, M. P. Hobson and A. N. Lasenby, PolyChord: nested sampling for cosmology, Mon. Not. Roy. Astron. Soc. 450 (2015) L61, [1502.01856].

[184] A. Lewis and A. Challinor, Weak gravitational lensing of the CMB, Phys. Rep. 429 (2006) 1, [astro-ph/0601594].

[185] B. D. Sherwin and M. Schmittfull, Delensing the CMB with the cosmic infrared background, Phy. Rev. D 92 (2015) 043005, [1502.05356]. 
[186] G. Simard, D. Hanson and G. Holder, Prospects for Delensing the Cosmic Microwave Background for Studying Inflation, Ap. J. 807 (2015) 166, [1410.0691].

[187] L. Marian and G. M. Bernstein, Detectability of CMB tensor B modes via delensing with weak lensing galaxy surveys, Phys. Rev. D 76 (2007) 123009, [0710.2538].

[188] U. Seljak and C. M. Hirata, Gravitational lensing as a contaminant of the gravity wave signal in the CMB, Phys. Rev. D 69 (2004) 043005, [astro-ph/0310163].

[189] M. Kesden, A. Cooray and M. Kamionkowski, Separation of Gravitational-Wave and Cosmic-Shear Contributions to Cosmic Microwave Background Polarization, Phys. Rev. Lett. 89 (2002) 011304, [astro-ph/0202434].

[190] L. Knox and Y.-S. Song, Limit on the Detectability of the Energy Scale of Inflation, Phys. Rev.Lett. 89 (2002) 011303, [astro-ph/0202286].

[191] K. M. Smith, D. Hanson, M. LoVerde, C. M. Hirata and O. Zahn, Delensing CMB polarization with external datasets, JCAP 6 (2012) 014, [1010.0048].

[192] T. Okamoto and W. Hu, Cosmic microwave background lensing reconstruction on the full sky, Phys. Rev. D 67 (2003) 083002, [astro-ph/0301031].

[193] J. Hamann, J. Lesgourgues and W. Valkenburg, How to constrain inflationary parameter space with minimal priors, JCAP 0804 (2008) 016, [0802.0505].

[194] F. Finelli, J. Hamann, S. M. Leach and J. Lesgourgues, Single-field inflation constraints from CMB and SDSS data, JCAP 1004 (2010) 011, [0912.0522].

[195] A. Kosowsky and M. S. Turner, CBR anisotropy and the running of the scalar spectral index, Phys. Rev. D52 (1995) R1739, [astro-ph/9504071].

[196] C. Pahud, A. R. Liddle, P. Mukherjee and D. Parkinson, When can the Planck satellite measure spectral index running?, Mon. Not. Roy. Astron. Soc. 381 (2007) 489, [astro-ph/0701481].

[197] P. Adshead, R. Easther, J. Pritchard and A. Loeb, Inflation and the Scale Dependent Spectral Index: Prospects and Strategies, JCAP 1102 (2011) 021, [1007.3748].

[198] Z. Hou et al., Constraints on Cosmology from the Cosmic Microwave Background Power Spectrum of the $2500 \mathrm{deg}^{2}$ SPT-SZ Survey, Astrophys. J. 782 (2014) 74, [1212.6267].

[199] G. Cabass, E. Di Valentino, A. Melchiorri, E. Pajer and J. Silk, Running the running, 1605.00209.

[200] A. Pourtsidou, Synergistic tests of inflation, 1612.05138.

[201] S. Ferrara and R. Kallosh, Seven-disk manifold, $\alpha$-attractors, and B modes, Phys. Rev. D94 (2016) 126015, [1610.04163].

[202] A. A. Starobinsky, A New Type of Isotropic Cosmological Models Without Singularity, Phys. Lett. B91 (1980) 99.

[203] V. F. Mukhanov and G. Chibisov, Quantum Fluctuation and Nonsingular Universe., JETP Lett. 33 (1981) 532.

[204] A. Starobinsky, The Perturbation Spectrum Evolving from a Nonsingular Initially de Sitter Cosmology and the Microwave Background Anisotropy, Sov. Astron. Lett. 9 (1983) 302.

[205] J. Martin, C. Ringeval, R. Trotta and V. Vennin, The Best Inflationary Models After Planck, JCAP 1403 (2014) 039, [1312.3529].

[206] S. V. Ketov and A. A. Starobinsky, Embedding $\left(R+R^{2}\right)$ Inflation into Supergravity, Phys. Rev. D83 (2011) 063512, [1011.0240]. 
[207] K. Freese, J. A. Frieman and A. V. Olinto, Natural inflation with pseudo-Nambu-Goldstone bosons, Phys. Rev. Lett. 65 (1990) 3233-3236.

[208] F. C. Adams, J. R. Bond, K. Freese, J. A. Frieman and A. V. Olinto, Natural inflation: Particle physics models, power law spectra for large scale structure, and constraints from COBE, Phys. Rev. D47 (1993) 426-455, [hep-ph/9207245].

[209] L. Boubekeur and D. Lyth, Hilltop inflation, JCAP 0507 (2005) 010, [hep-ph/0502047].

[210] J. Garriga and V. F. Mukhanov, Perturbations in k-inflation, Phys. Lett. B 458 (1999) 219, [arXiv:hep-th/9904176].

[211] T. Kobayashi, M. Yamaguchi and J. Yokoyama, G-inflation: Inflation driven by the Galileon field, Phys. Rev. Lett. 105 (2010) 231302, [1008.0603].

[212] N. Bartolo, S. Matarrese and A. Riotto, Adiabatic and isocurvature perturbations from inflation: Power spectra and consistency relations, Phys. Rev. D64 (2001) 123504, [astro-ph/0107502].

[213] D. Wands, N. Bartolo, S. Matarrese and A. Riotto, An Observational test of two-field inflation, Phys. Rev. D66 (2002) 043520, [astro-ph/0205253].

[214] C. T. Byrnes and D. Wands, Curvature and isocurvature perturbations from two-field inflation in a slow-roll expansion, Phys. Rev. D74 (2006) 043529, [astro-ph/0605679].

[215] A. Maleknejad, M. M. Sheikh-Jabbari and J. Soda, Gauge Fields and Inflation, Phys. Rept. 528 (2013) 161, [1212.2921].

[216] N. Barnaby and M. Peloso, Large Nongaussianity in Axion Inflation, Phys. Rev. Lett. 106 (2011) 181301, [1011.1500].

[217] C. Guzzetti, M., N. Bartolo, M. Liguori and S. Matarrese, Gravitational waves from inflation, Riv. Nuovo Cim. 39 (2016) 399-495, [1605.01615].

[218] M. Gasperini and G. Veneziano, Pre - big bang in string cosmology, Astropart. Phys. 1 (1993) 317, [hep-th/9211021].

[219] L. A. Boyle, P. J. Steinhardt and N. Turok, The Cosmic gravitational wave background in a cyclic universe, Phys. Rev. D69 (2004) 127302, [hep-th/0307170].

[220] R. H. Brandenberger, A. Nayeri, S. P. Patil and C. Vafa, Tensor Modes from a Primordial Hagedorn Phase of String Cosmology, Phys. Rev. Lett. 98 (2007) 231302, [hep-th/0604126].

[221] Q.-G. Huang, S. Wang and W. Zhao, Forecasting sensitivity on tilt of power spectrum of primordial gravitational waves after Planck satellite, JCAP 1510 (2015) 035, [1509.02676].

[222] M. Kleban and M. Schillo, Spatial Curvature Falsifies Eternal Inflation, JCAP 1206 (2012) 029, [1202.5037].

[223] Planck collaboration, P. A. R. Ade et al., Planck 2013 results. I. Overview of products and scientific results, Astron. Astrophys. 571 (2014) A1, [1303.5062].

[224] Planck collaboration, R. Adam et al., Planck 2015 results. I. Overview of products and scientific results, 1502.01582.

[225] V. Vennin, J. Martin and C. Ringeval, Cosmic Inflation and Model Comparison, Comptes Rendus Physique (2015) .

[226] D. Blas, J. Lesgourgues and T. Tram, The Cosmic Linear Anisotropy Solving System (CLASS). Part II: Approximation schemes, JCAP 7 (2011) 034, [1104.2933]. 
[227] B. Audren, J. Lesgourgues, K. Benabed and S. Prunet, Conservative constraints on early cosmology with MONTE PYTHON, JCAP 2 (2013) 001, [1210.7183].

[228] J. Martin, C. Ringeval and V. Vennin, Information Gain on Reheating: The One Bit Milestone, 1603.02606.

[229] R. T. Cox, Probability, Frequency and Reasonable Expectation, Am. J. of Phy. 14 (Jan 1946) 1.

[230] H. Jeffreys, Theory of probability, Oxford University Press (reprinted 1998) (1961) .

[231] B. de Finetti, Theory of probability, John Wiley $\&$ Sons, Chichester, UK (reprinted 1974) (1995) .

[232] R. Trotta, Bayes in the sky: Bayesian inference and model selection in cosmology, Contemp. Phys. 49 (2008) 71, [0803.4089].

[233] C. Ringeval, Fast Bayesian inference for slow-roll inflation, Mon. Not. Roy. Astron. Soc. 439 (2014) 3253, [1312.2347].

[234] J. Martin, C. Ringeval, R. Trotta and V. Vennin, Compatibility of Planck and BICEP2 in the Light of Inflation, Phys. Rev. D90 (2014) 063501, [1405.7272].

[235] J. Martin, C. Ringeval and V. Vennin, How Well Can Future CMB Missions Constrain Cosmic Inflation?, JCAP 1410 (2014) 038, [1407.4034].

[236] V. Vennin, K. Koyama and D. Wands, Inflation with an extra light scalar field after Planck, JCAP 1603 (2016) 024, [1512.03403].

[237] R. J. Hardwick, V. Vennin, K. Koyama and D. Wands, Constraining Curvatonic Reheating, JCAP 1608 (2016) 042, [1606.01223].

[238] J. Martin, C. Ringeval and V. Vennin, "Accurate Slow-Roll Predictions for Inflationary Cosmology." http://cp3.irmp.ucl.ac.be/ ringeval/aspic.html.

[239] P. Mukherjee, D. Parkinson and A. R. Liddle, A nested sampling algorithm for cosmological model selection, Astrophys. J. 638 (2006) L51, [astro-ph/0508461].

[240] J. Martin and C. Ringeval, Inflation after WMAP3: Confronting the Slow-Roll and Exact Power Spectra to CMB Data, JCAP 0608 (2006) 009, [astro-ph/0605367].

[241] J. Martin and C. Ringeval, First CMB Constraints on the Inflationary Reheating Temperature, Phys.Rev. D82 (2010) 023511, [1004.5525].

[242] R. Easther and H. V. Peiris, Bayesian Analysis of Inflation II: Model Selection and Constraints on Reheating, Phys.Rev. D85 (2012) 103533, [1112.0326].

[243] L. Dai, M. Kamionkowski and J. Wang, Reheating constraints to inflationary models, Phys.Rev.Lett. 113 (2014) 041302, [1404.6704].

[244] T. Rehagen and G. B. Gelmini, Low reheating temperatures in monomial and binomial inflationary potentials, JCAP 1506 (2015) 039, [1504.03768].

[245] J. Martin, C. Ringeval and V. Vennin, Observing Inflationary Reheating, Phys. Rev. Lett. 114 (2015) 081303, [1410.7958].

[246] S. Kullback and R. A. Leibler, On information and sufficiency, Ann. Math. Statist. 22 (1951) 79.

[247] M. Kunz, R. Trotta and D. Parkinson, Measuring the effective complexity of cosmological models, Phys. Rev. D74 (2006) 023503, [astro-ph/0602378].

[248] A. R. Liddle, Information criteria for astrophysical model selection, Mon. Not. Roy. Astron. Soc. 377 (2007) L74, [astro-ph/0701113]. 
[249] J. M. Bernardo and A. F. M. Smith, Bayesian Theory, p. 105. John Wiley \& Sons, Inc., 2008. $10.1002 / 9780470316870$.

[250] M. Drewes, What can the CMB tell about the microphysics of cosmic reheating?, JCAP 1603 (2016) 013, [1511.03280].

[251] S. Renaux-Petel and K. Turzy?ski, Geometrical Destabilization of Inflation, Phys. Rev. Lett. 117 (2016) 141301, [1510.01281].

[252] J. Martin, C. Ringeval and V. Vennin, Shortcomings of New Parametrizations of Inflation, 1609.04739.

[253] T. Terada, Y. Watanabe, Y. Yamada and J. Yokoyama, Reheating processes after Starobinsky inflation in old-minimal supergravity, JHEP 02 (2015) 105, [1411.6746].

[254] C. Gauthier and M. Bucher, Reconstructing the primordial power spectrum from the CMB, JCAP 10 (2012) 050, [1209.2147].

[255] J. A. Vázquez, M. Bridges, M. P. Hobson and A. N. Lasenby, Model selection applied to reconstruction of the Primordial Power Spectrum, JCAP 6 (2012) 006, [1203.1252].

[256] G. Aslanyan, L. C. Price, K. N. Abazajian and R. Easther, The Knotted Sky I: Planck constraints on the primordial power spectrum, JCAP 8 (2014) 052, [1403.5849].

[257] W. J. Handley, M. P. Hobson and A. N. Lasenby, POLYCHORD: nested sampling for cosmology, MNRAS 450 (2015) L61, [1502.01856].

[258] W. J. Handley, M. P. Hobson and A. N. Lasenby, POLYCHORD: next-generation nested sampling, MNRAS 453 (2015) 4384, [1506.00171].

[259] S. Hee, J. A. Vázquez, W. J. Handley, M. P. Hobson and A. N. Lasenby, Constraining the dark energy equation of state using Bayes theorem and the Kullback-Leibler divergence, ArXiv e-prints (2016) , [1607.00270].

[260] D. K. Hazra, A. Shafieloo and G. F. Smoot, Reconstruction of broad features in the primordial spectrum and inflaton potential from planck, JCAP 2013 (2013) 035.

[261] B. Hu, J.-W. Hu, Z.-K. Guo and R.-G. Cai, Reconstruction of the primordial power spectra with Planck and BICEP2 data, Phys. Rev. D 90 (2014) 023544, [1404.3690].

[262] A. Ravenni, L. Verde and A. J. Cuesta, Red, Straight, no bends: primordial power spectrum reconstruction from CMB and large-scale structure, JCAP 8 (2016) 028, [1605.06637].

[263] S. Gariazzo, O. Mena, H. Ramirez and L. Boubekeur, Primordial power spectrum features in phenomenological descriptions of inflation, ArXiv e-prints (2016), [1606.00842].

[264] D. K. Hazra, A. Shafieloo, G. F. Smoot and A. A. Starobinsky, Primordial features and planck polarization, JCAP 2016 (2016) 009.

[265] D. K. Hazra, A. Shafieloo and T. Souradeep, Primordial power spectrum from planck, JCAP 2014 (2014) 011.

[266] C. R. Contaldi, M. Peloso, L. Kofman and A. Linde, Suppressing the lower multipoles in the CMB anisotropies, JCAP 7 (2003) 2.

[267] R. Flauger, L. McAllister, E. Pajer, A. Westphal and G. Xu, Oscillations in the CMB from Axion Monodromy Inflation, JCAP 1006 (2010) 009, [0907.2916].

[268] M. Ballardini, F. Finelli, C. Fedeli and L. Moscardini, Probing primordial features with future galaxy surveys, JCAP 10 (2016) 041, [1606.03747]. 
[269] D. Langlois, Correlated adiabatic and isocurvature perturbations from double inflation, Phys. Rev. D59 (1999) 123512, [astro-ph/9906080].

[270] D. Langlois and A. Riazuelo, Correlated mixtures of adiabatic and isocurvature cosmological perturbations, Phys. Rev. D62 (2000) 043504, [astro-ph/9912497].

[271] C. Gordon, D. Wands, B. A. Bassett and R. Maartens, Adiabatic and entropy perturbations from inflation, Phys. Rev. D63 (2001) 023506, [astro-ph/0009131].

[272] L. Amendola, C. Gordon, D. Wands and M. Sasaki, Correlated perturbations from inflation and the cosmic microwave background, Phys. Rev. Lett. 88 (2002) 211302, [astro-ph/0107089].

[273] M. Beltran, J. Garcia-Bellido, J. Lesgourgues and A. Riazuelo, Bounds on CDM and neutrino isocurvature perturbations from CMB and LSS data, Phys. Rev. D70 (2004) 103530, [astro-ph/0409326].

[274] M. Beltran, J. Garcia-Bellido, J. Lesgourgues, A. R. Liddle and A. Slosar, Bayesian model selection and isocurvature perturbations, Phys. Rev. D71 (2005) 063532, [astro-ph/0501477].

[275] M. Savelainen, J. Valiviita, P. Walia, S. Rusak and H. Kurki-Suonio, Constraints on neutrino density and velocity isocurvature modes from WMAP-9 data, Phys. Rev. D88 (2013) 063010, [1307.4398].

[276] D. Grin, D. Hanson, G. P. Holder, O. Dore and M. Kamionkowski, Baryons do trace dark matter 380,000 years after the big bang: Search for compensated isocurvature perturbations with WMAP 9-year data, Phys. Rev. D89 (2014) 023006, [1306.4319].

[277] D. Grin, O. Dore and M. Kamionkowski, Compensated Isocurvature Perturbations and the Cosmic Microwave Background, Phys. Rev. D84 (2011) 123003, [1107.5047].

[278] J. B. Muñoz, D. Grin, L. Dai, M. Kamionkowski and E. D. Kovetz, Search for Compensated Isocurvature Perturbations with Planck Power Spectra, Phys. Rev. D93 (2016) 043008, [1511.04441].

[279] J. Valiviita, Power Spectra Based Planck Constraints on Compensated Isocurvature, and Forecasts for LiteBIRD and CORE Space Missions, 1701.07039.

[280] H. Kurki-Suonio, V. Muhonen and J. Valiviita, Correlated primordial perturbations in light of CMB and LSS data, Phys. Rev. D71 (2005) 063005, [astro-ph/0412439].

[281] C. J. MacTavish et al., Cosmological parameters from the 2003 flight of BOOMERANG, Astrophys. J. 647 (2006) 799, [astro-ph/0507503].

[282] R. Keskitalo, H. Kurki-Suonio, V. Muhonen and J. Valiviita, Hints of Isocurvature Perturbations in the Cosmic Microwave Background, JCAP 0709 (2007) 008, [astro-ph/0611917].

[283] Planck collaboration, J. Tauber, M. Bersanelli, J. M. Lamarre, G. Efstathiou, C. Lawrence, F. Bouchet et al., The Scientific programme of Planck, astro-ph/0604069.

[284] P. Crotty, J. Garcia-Bellido, J. Lesgourgues and A. Riazuelo, Bounds on isocurvature perturbations from CMB and LSS data, Phys. Rev. Lett. 91 (2003) 171301, [astro-ph/0306286].

[285] J. Valiviita and T. Giannantonio, Constraints on primordial isocurvature perturbations and spatial curvature by Bayesian model selection, Phys. Rev. D80 (2009) 123516, [0909.5190].

[286] M. Beltran, J. Garcia-Bellido and J. Lesgourgues, Isocurvature bounds on axions revisited, Phys. Rev. D75 (2007) 103507, [hep-ph/0606107].

[287] C. Gordon and A. Lewis, Observational constraints on the curvaton model of inflation, Phys. Rev. D67 (2003) 123513, [astro-ph/0212248]. 
[288] J. Valiviita, M. Savelainen, M. Talvitie, H. Kurki-Suonio and S. Rusak, Constraints on scalar and tensor perturbations in phenomenological and two-field inflation models: Bayesian evidences for primordial isocurvature and tensor modes, Astrophys. J. 753 (2012) 151, [1202.2852].

[289] M. Bucher, B. Racine and B. van Tent, The binned bispectrum estimator: template-based and non-parametric CMB non-Gaussianity searches, JCAP 1605 (2016) 055, [1509.08107].

[290] E. Komatsu and D. N. Spergel, Acoustic signatures in the primary microwave background bispectrum, Phys. Rev. D 63 (2001) 063002, [astro-ph/0005036].

[291] J. R. Fergusson, M. Liguori and E. P. S. Shellard, General CMB and primordial bispectrum estimation: Mode expansion, map making, and measures of $F_{N L}$, Phys. Rev. D 82 (2010) 023502, [0912.5516].

[292] D. Langlois and A. Lepidi, General treatment of isocurvature perturbations and non-Gaussianities, JCAP 1101 (2011) 008, [1007.5498].

[293] D. Langlois and B. van Tent, Hunting for Isocurvature Modes in the CMB non-Gaussianities, Class.Quant.Grav. 28 (2011) 222001, [1104.2567].

[294] D. Langlois and B. van Tent, Isocurvature modes in the CMB bispectrum, JCAP 1207 (2012) 040, [1204.5042].

[295] M. LoVerde, A. Miller, S. Shandera and L. Verde, Effects of Scale-Dependent Non-Gaussianity on Cosmological Structures, JCAP 0804 (2008) 014, [0711.4126].

[296] A. Taruya, K. Koyama and T. Matsubara, Signature of primordial non-Gaussianity on the matter power spectrum, Phys. Rev. D 78 (2008) 123534, [0808.4085].

[297] A. Becker and D. Huterer, First Constraints on the Running of Non-Gaussianity, Phys. Rev. Lett. 109 (2012) 121302, [1207.5788].

[298] J. R. Fergusson, H. F. Gruetjen, E. P. S. Shellard and B. Wallisch, Polyspectra searches for sharp oscillatory features in cosmic microwave sky data, Phys. Rev. D 91 (2015) 123506, [1412.6152].

[299] P. D. Meerburg, M. Münchmeyer and B. Wandelt, Joint resonant CMB power spectrum and bispectrum estimation, Phys. Rev. D 93 (2016) 043536, [1510.01756].

[300] A. Achúcarro, V. Atal, P. Ortiz and J. Torrado, Localized correlated features in the CMB power spectrum and primordial bispectrum from a transient reduction in the speed of sound, Phys. Rev. D89 (2014) 103006, [1311.2552].

[301] J. Torrado, B. Hu and A. Achucarro, Robust predictions for an oscillatory bispectrum in Planck 2015 data from transient reductions in the speed of sound of the inflaton, 1611.10350.

[302] T. Sekiguchi and N. Sugiyama, Optimal constraint on $g_{N L}$ from CMB, JCAP 1309 (2013) 002, [1303.4626].

[303] K. M. Smith, L. Senatore and M. Zaldarriaga, Optimal analysis of the CMB trispectrum, ArXiv e-prints (2015), [1502.00635].

[304] Y. B. Zeldovich and R. A. Sunyaev, The Interaction of Matter and Radiation in a Hot-Model Universe, ApSS 4 (1969) 301.

[305] R. A. Sunyaev and Y. B. Zeldovich, The interaction of matter and radiation in the hot model of the Universe, II, ApSS 7 (1970) 20.

[306] C. Burigana, L. Danese and G. de Zotti, Formation and evolution of early distortions of the microwave background spectrum - A numerical study, A\&A 246 (1991) 49. 
[307] W. Hu and J. Silk, Thermalization and spectral distortions of the cosmic background radiation, Phys. Rev. D 48 (1993) 485.

[308] J. Chluba and R. A. Sunyaev, The evolution of CMB spectral distortions in the early Universe, MNRAS 419 (2012) 1294, [1109.6552].

[309] M. Tucci, V. Desjacques and M. Kunz, Cosmic Infrared Background anisotropies as a window into primordial non-Gaussianity, ArXiv e-prints (2016) , [1606.02323].

[310] E. Pajer and M. Zaldarriaga, A New Window on Primordial non-Gaussianity, Phys. Rev. Lett. 109 (2012) 021302, [1201.5375].

[311] M. Biagetti, H. Perrier, A. Riotto and V. Desjacques, Testing the running of non-Gaussianity through the CMB $\mu$-distortion and the halo bias, Phys. Rev. D 87 (2013) 063521, [1301.2771].

[312] J. Ganc and E. Komatsu, Scale-dependent bias of galaxies and mu-type distortion of the cosmic microwave background spectrum from single-field inflation with a modified initial state, Phys. Rev. D86 (2012) 023518, [1204.4241].

[313] R. Emami, E. Dimastrogiovanni, J. Chluba and M. Kamionkowski, Probing the scale dependence of non-Gaussianity with spectral distortions of the cosmic microwave background, Phys. Rev. D 91 (2015) 123531, [1504.00675].

[314] M. Shiraishi, M. Liguori, N. Bartolo and S. Matarrese, Measuring primordial anisotropic correlators with CMB spectral distortions, Phys. Rev. D 92 (2015) 083502, [1506. 06670].

[315] N. Bartolo, M. Liguori and M. Shiraishi, Primordial trispectra and CMB spectral distortions, JCAP 3 (2016) 029, [1511.01474].

[316] J. Chluba, E. Dimastrogiovanni, M. A. Amin and M. Kamionkowski, Evolution of CMB spectral distortion anisotropies and tests of primordial non-Gaussianity, ArXiv e-prints (2016), [1610.08711].

[317] Planck Collaboration XXX, Planck 2013 results. XXX. Cosmic infrared background measurements and implications for star formation, A\&A 571 (2014) A30, [1309.0382].

[318] M. Tegmark, D. J. Eisenstein, W. Hu and A. de Oliveira-Costa, Foregrounds and Forecasts for the Cosmic Microwave Background, ApJ 530 (2000) 165, [astro-ph/9905257].

[319] R. A. Sunyaev and Y. B. Zeldovich, Small scale entropy and adiabatic density perturbations Antimatter in the Universe, ApSS 9 (1970) 368.

[320] R. A. Daly, Spectral distortions of the microwave background radiation resulting from the damping of pressure waves, ApJ 371 (1991) 14.

[321] W. Hu, D. Scott and J. Silk, Power spectrum constraints from spectral distortions in the cosmic microwave background, ApJL 430 (1994) L5, [arXiv:astro-ph/9402045].

[322] J. Chluba, R. Khatri and R. A. Sunyaev, CMB at $2 \times 2$ order: the dissipation of primordial acoustic waves and the observable part of the associated energy release, MNRAS 425 (2012) 1129, [1202.0057].

[323] I. Agullo and L. Parker, Non-Gaussianities and the stimulated creation of quanta in the inflationary universe, Phys. Rev. D 83 (2011) 063526, [1010.5766].

[324] J. Ganc, Calculating the local-type $f_{N L}$ for slow-roll inflation with a nonvacuum initial state, Phys. Rev. D 84 (2011) 063514, [1104.0244].

[325] M. Tellarini, A. J. Ross, G. Tasinato and D. Wands, Galaxy bispectrum, primordial non-Gaussianity and redshift space distortions, JCAP 6 (2016) 014, [1603.06814]. 
[326] N. Agarwal, S. Ho and S. Shandera, Constraining the initial conditions of the Universe using large scale structure, JCAP 1402 (2014) 038, [1311.2606].

[327] R. de Putter and O. Doré, Designing an Inflation Galaxy Survey: how to measure $\sigma\left(f_{\mathrm{NL}}\right) \sim 1$ using scale-dependent galaxy bias, 1412.3854.

[328] S. Camera, M. G. Santos and R. Maartens, Probing primordial non-Gaussianity with SKA galaxy redshift surveys: a fully relativistic analysis, Mon. Not. Roy. Astron. Soc. 448 (2015) 1035, [1409.8286].

[329] T. Baldauf, M. Mirbabayi, M. Simonović and M. Zaldarriaga, LSS constraints with controlled theoretical uncertainties, ArXiv e-prints (2016), [1602.00674].

[330] J. B. Muñoz, Y. Ali-Haïmoud and M. Kamionkowski, Primordial non-gaussianity from the bispectrum of 21-cm fluctuations in the dark ages, Phys. Rev. D 92 (2015) 083508, [1506.04152].

[331] P. Mukherjee, J. Urrestilla, M. Kunz, A. R. Liddle, N. Bevis et al., Detecting and distinguishing topological defects in future data from the CMBPol satellite, Phys.Rev. D83 (2011) 043003, [1010.5662].

[332] U.-L. Pen, U. Seljak and N. Turok, Power spectra in global defect theories of cosmic structure formation, Phys.Rev.Lett. 79 (1997) 1611, [astro-ph/9704165].

[333] R. Durrer, M. Kunz and A. Melchiorri, Cosmic structure formation with topological defects, Phys.Rept. 364 (2002) 1, [astro-ph/0110348].

[334] L. Pogosian and T. Vachaspati, Cosmic microwave background anisotropy from wiggly strings, Phys.Rev. D60 (1999) 083504, [astro-ph/9903361].

[335] T. Charnock, A. Avgoustidis, E. J. Copeland and A. Moss, CMB constraints on cosmic strings and superstrings, Phys. Rev. D93 (2016) 123503, [1603.01275].

[336] A. Achúcarro, A. Avgoustidis, A. Leite, A. Lopez-Eiguren, C. Martins et al., Evolution of Semilocal String Networks: I. Large-scale Properties, Phys.Rev. D89 (2014) 063503, [1312.2123].

[337] D. Daverio, M. Hindmarsh, M. Kunz, J. Lizarraga and J. Urrestilla, Energy-momentum correlations for Abelian Higgs cosmic strings, Phys. Rev. D93 (2016) 085014, [1510.05006].

[338] J. Lizarraga, J. Urrestilla, D. Daverio, M. Hindmarsh and M. Kunz, New CMB constraints for Abelian Higgs cosmic strings, JCAP 1610 (2016) 042, [1609.03386].

[339] J. Urrestilla, N. Bevis, M. Hindmarsh and M. Kunz, Cosmic string parameter constraints and model analysis using small scale Cosmic Microwave Background data, JCAP 1112 (2011) 021, [1108.2730].

[340] Planck collaboration, P. Ade et al., Planck 2013 results. XXV. Searches for cosmic strings and other topological defects, Astron.Astrophys. 571 (2014) A25, [1303.5085]. 